\title{
Using the internet to promote a healthy lifestyle: testing a multiple behaviour change intervention regarding reach, use, appreciation, effectiveness and cost-effectiveness
}

Citation for published version (APA):

Schulz, D. N. (2014). Using the internet to promote a healthy lifestyle: testing a multiple behaviour change intervention regarding reach, use, appreciation, effectiveness and cost-effectiveness. [Doctoral Thesis, Maastricht University]. Maastricht University. https://doi.org/10.26481/dis.20140528ds

Document status and date:

Published: 01/01/2014

DOI:

$10.26481 /$ dis.20140528ds

Document Version:

Publisher's PDF, also known as Version of record

Please check the document version of this publication:

- A submitted manuscript is the version of the article upon submission and before peer-review. There can be important differences between the submitted version and the official published version of record. People interested in the research are advised to contact the author for the final version of the publication, or visit the DOI to the publisher's website.

- The final author version and the galley proof are versions of the publication after peer review.

- The final published version features the final layout of the paper including the volume, issue and page numbers.

Link to publication

\footnotetext{
General rights rights.

- You may freely distribute the URL identifying the publication in the public portal. please follow below link for the End User Agreement:

www.umlib.nl/taverne-license

Take down policy

If you believe that this document breaches copyright please contact us at:

repository@maastrichtuniversity.nl

providing details and we will investigate your claim.
}

Copyright and moral rights for the publications made accessible in the public portal are retained by the authors and/or other copyright owners and it is a condition of accessing publications that users recognise and abide by the legal requirements associated with these

- Users may download and print one copy of any publication from the public portal for the purpose of private study or research.

- You may not further distribute the material or use it for any profit-making activity or commercial gain

If the publication is distributed under the terms of Article 25fa of the Dutch Copyright Act, indicated by the "Taverne" license above, 


\section{USING THE INTERNET TO PROMOTE A HEALTHY LIFESTYLE}

TESTING A MULTIPLE BEHAVIOUR CHANGE INTERVENTION REGARDING

REACH, USE, APPRECIATION, EFFECTIVENESS AND COST-EFFECTIVENESS

Daniela N. Schulz 


\section{COLOPHON}

Cover design and layout: Dirk J. Veldman | Daniela N. Schulz

Production: Ridderprint BV, Ridderkerk, The Netherlands

(C) Daniela N. Schulz, 2014

ISBN: 978-90-5335-837-5

The research presented in this dissertation was conducted at the School for Public Health and Primary Care (CAPHRI), Department of Health Promotion, of Maastricht University. CAPHRI participates in CaRe, the Netherlands School of Primary Care Research. CAPHRI was classified as 'excellent' by the external evaluation committee of leading international experts that reviewed CAPHRI in December 2010.

The research described in this thesis was funded by the Netherlands Organization for Health Research and Development (ZonMw, Grant No. 120610012).

All rights are reserved. No part of this book may be reproduced or transmitted in any form or by any means, without the written permission from the author or, where appropriate, the publisher of the article. 


\section{USING THE INTERNET TO PROMOTE A HEALTHY LIFESTYLE}

TESTING A MULTIPLE BEHAVIOUR CHANGE INTERVENTION REGARDING

REACH, USE, APPRECIATION, EFFECTIVENESS AND COST-EFFECTIVENESS

PROEFSCHRIFT

ter verkrijging van de graad van doctor aan de Universiteit Maastricht, op gezag van de Rector Magnificus, Prof. dr. L.L.G. Soete, volgens het besluit van het College van Decanen, in het openbaar te verdedigen op woensdag 28 mei 2014 om 12.00 uur door

Daniela Nadine Schulz geboren op 20 februari 1984 te Duisburg (Duitsland) 


\section{PROMOTORES}

Prof. dr. H. de Vries

Prof. dr. S.P.J. Kremers

\section{BEOORDELINGSCOMMISSIE}

Prof. dr. N.K. de Vries (voorzitter)

Prof. dr. I. de Bourdeaudhuij, Universiteit Gent

Dr. R. Crutzen

Prof. dr. L. Lechner, Open Universiteit

Prof. dr. L. de Witte 
CHAPTER 1

GENERAL INTRODUCTION

CHAPTER 2

TESTING A DUTCH WEB-BASED TAILORED LIFESTYLE PROGRAMME AMONG ADULTS: A STUDY PROTOCOL

\section{CHAPTER 3}

THE USE OF A PROACTIVE DISSEMINATION STRATEGY TO OPTIMIZE REACH OF AN INTERNET-DELIVERED COMPUTER-TAILORED LIFESTYLE INTERVENTION

\section{CHAPTER 4}

PROGRAMME COMPLETION OF A WEB-BASED TAILORED LIFESTYLE INTERVENTION FOR ADULTS: DIFFERENCES BETWEEN A SEQUENTIAL AND A SIMULTANEOUS APPROACH

\section{CHAPTER 5}

TAILORED EHEALTH LIFESTYLE PROMOTION: WHICH BEHAVIOURAL MODULES DO USERS PREFER?

\section{CHAPTER 6}

EFFECTS OF A WEB-BASED TAILORED INTERVENTION TO REDUCE ALCOHOL CONSUMPTION IN ADULTS:

RANDOMIZED CONTROLLED TRIAL

\section{CHAPTER 7}

EFFECTS OF A WEB-BASED TAILORED MULTIPLE LIFESTYLE INTERVENTION FOR ADULTS: A TWO-YEAR RANDOMIZED CONTROLLED TRIAL COMPARING SEQUENTIAL AND SIMULTANEOUS DELIVERY MODES

\section{CHAPTER 8}

ECONOMIC EVALUATION OF A WEB-BASED TAILORED LIFESTYLE INTERVENTION FOR ADULTS: FINDINGS REGARDING COST-EFFECTIVENESS AND COST-UTILITY FROM A RANDOMIZED CONTROLLED TRIAL

\section{CHAPTER 9}

GENERAL DISCUSSION

REFERENCES

APPENDICES

SUMMARY

SAMENVATTING

ZUSAMMENFASSUNG

DANKWOORD

CURRICULUM VITAE 



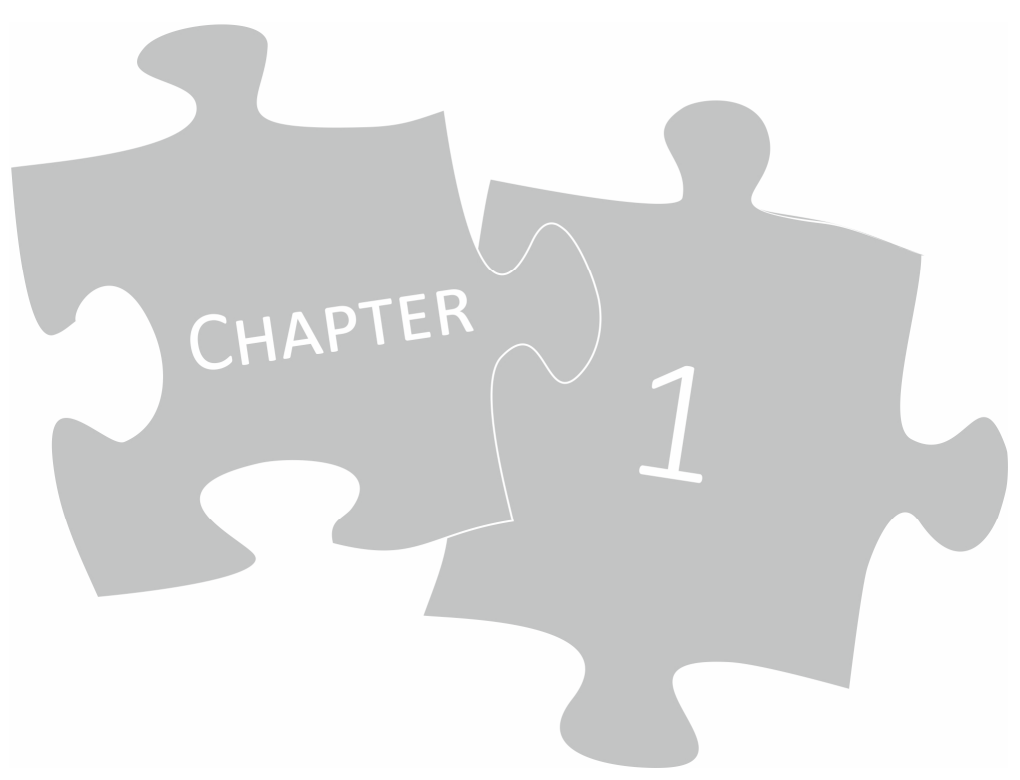

GENERAL INTRODUCTION 



\section{INTRODUCTION}

There has been a rapid increase in Internet use in recent years (Internet World Stats, 2012). Nowadays, people communicate via e-mail, social network sites or mobile applications; they download movies instead of going to the cinema or watch movies through online streaming; they manage their finances via the Internet; and online shopping is increasingly popular as is searching for information on any topic. The Internet is used during both the working day and in leisure time. In more and more countries, including the Netherlands, the time spent on Internet use has even exceeded that spent watching television (TNS, 2012).

The Internet is also used to search for health information (Moretti et al., 2012; Online Health Search, 2006). Approximately $60-80 \%$ of Internet users have looked up medical information on the web at least once (Atkinson et al., 2009; Fox, 2011; Online Health Search, 2006). This implies that the Internet can be an important access point for health promotion and disease prevention through its ability to reach many people. At present, more than $90 \%$ of the Dutch population has Internet access (Internet World Stats, 2012). Thus, the reach of Internet interventions is potentially large. However, one hindering factor is the high dropout rate in Internet interventions aimed at health promotion (Eysenbach, 2005). Further research is needed on how the Internet can be best used for prevention purposes, as people may be more motivated to look for information when they have complaints than when they are healthy.

In the Netherlands, approximately $25 \%$ of the inhabitants have one or more chronic diseases (Hoeymans et al., 2009), with diabetes, osteoarthritis, coronary heart diseases and cancer being the most prevalent (Gommer \& Poos, 2010). In general, chronic illnesses are more prevalent among older people (Hoeymans et al., 2009) and those with a lower socioeconomic status (Zantinge \& Uiters, 2009). Approximately $8 \%$ suffer from co-morbidity, such as diabetes and coronary heart diseases, or depression and anxiety disorders. Chronic diseases are often related to a lower quality of life (Hoeymans et al., 2009). Moreover, around $88 \%$ of all deaths in the Netherlands can be ascribed to chronic diseases, with most deaths caused by cardiovascular diseases (35\%) and cancer (29\%) (World Health Organization, 2002).

A person's chance of developing one or more chronic diseases can be reduced by motivating them to engage in healthy lifestyle behaviours, such as being physically active, eating sufficient fruit and vegetables, limiting alcohol consumption and not smoking (Doll \& Peto, 1981; World Health Organization, 2002). In the Netherlands, the following guidelines exist for the behaviours mentioned above: being physically active at least five days a week for at least 30 minutes (Hildebrandt et al., 2010); eating 200 grams of vegetables per day (The Health Council of the Netherlands, 2006); eating two pieces of fruit per day (The Health Council of the Netherlands, 2006); consuming no more than one (women) or two (men) alcoholic drinks per day (The Health Council of the Netherlands, 2006); and not smoking (The Dutch Institute for Healthcare Improvement, 2004).

In the Netherlands, an unhealthy lifestyle is common among adults. In particular, the percentage of people complying with the guidelines for fruit and vegetable intake is low, with less than $10 \%$ of the 
Dutch population eating sufficient amounts of fruit or vegetables (Van Rossum et al., 2011). Higher educated adults seem to adhere to the guidelines for fruit and vegetable intake more often than lower educated adults (Geurts \& Van Rossum, 2013; Van Rossum et al., 2011). With regards to physical activity, around $40 \%$ of the population do not comply with the guideline; among the younger and older generations, men comply with the guideline more frequently, whereas among the middle-aged population, women comply with the guideline more (Hildebrandt et al., 2013; Statistics Netherlands, 2013b; Wendel-Vos, 2013). Moreover, people with a lower socioeconomic status adhere to the physical activity guideline more often, whereas those with a higher socioeconomic status report participating in sports more regularly (Ooijendijk et al., 2007; Statistics Netherlands, 2007b; Uiters \& Verweij, 2013). According to the Dutch Expert Centre for Tobacco Control, approximately 26\% of the Dutch population smokes with a higher percentage of smokers and ex-smokers among men and among people with a lower socioeconomic status (Stivoro, 2013). More than $80 \%$ of all people older than 12 years old report that they drink alcohol; 10\% can be described as heavy drinkers (i.e. consuming six or more glasses of alcohol at least once per week) (Statistics Netherlands, 2013b). Alcohol use seems to be more prevalent among higher educated people whereas heavy alcohol use seems to be more prevalent among those with a lower education (Savelkoul et al., 2011; Statistics Netherlands, 2009). Men are more likely to consume a higher amount of alcohol than women, and alcohol disorders are also more prevalent among men than among women (Netherlands Institute of Mental Health and Addiction, 2013b). Due to the high prevalence of unhealthy lifestyle behaviours and the association of an unhealthy lifestyle with a reduced quality of life and mortality risk, primary prevention in this area is important (Walter et al., 2012).

\section{The need for multiple behaviour change}

Since the main causes of morbidity and early mortality in Western countries - such as heart disease, cancer and stroke - are influenced by multiple health risk behaviours (Prochaska et al., 2008), and since these unhealthy behaviours often co-occur (De Vries et al., 2008c; Fine et al., 2004; Pronk et al., 2004b; Van Oort et al., 2004), it may be advisable to aim at changing multiple risk behaviours instead of just a single behaviour. When risk behaviours cluster, the negative effects on health are likely to be even greater (Kushner \& Sorensen, 2013). This implies that people with multiple unhealthy lifestyle behaviours are at highest risk of negative consequences. Studies have reported that smokers in particular have a higher likelihood of unhealthy behavioural profiles, with around $92 \%$ of them showing one or more additional risk behaviours (Fine et al., 2004; Pronk et al., 2004a). There tends to be a strong association between tobacco use and alcohol use (e.g. Jensen et al., 2003; Revicki et al., 1991; Rust et al., 2001; Van Oort et al., 2004). Poortinga (2007), for example, found that tobacco use and alcohol use also cluster with lack of fruit and vegetable consumption. Furthermore, in their study, physical activity clustered with smoking and drinking, whereas lack of physical activity clustered with lack of fruit and vegetable consumption. These different clusters give rise to the development of interventions that aim to induce multiple behaviour change. Multiple behaviour change would potentially result in increased health benefits, since a combination of healthy behaviours is likely to 
have a greater positive effect on health than a single health behaviour change, maximized health promotion and reduced health care costs due to less health care consumption (Prochaska et al., 2008). For example, if one quits smoking, increases their vegetable and fruit consumption and engages in more physical activity, this has a positive effect on cardiovascular risk reduction (Perk et al., 2012). In the last few years, various multiple behaviour change interventions, which were often delivered via the telephone or the World Wide Web, have been developed and had their effectiveness tested (e.g. Hawkes et al., 2013; Hyman et al., 2007; Vandelanotte et al., 2008; Van Genugten et al., 2012; Van Keulen et al., 2011; Velicer et al., 2013). The majority of these programmes were successful in reaching behavioural change. The assumption that certain behaviours may share various social and psychological determinants that are required for engaging in behavioural change (e.g. attitude or social influence) (Kremers et al., 2004; Noar et al., 2008), emphasizes one more advantage of a multiple behaviour change approach.

\section{Computer-tailoring}

One method of stimulating a healthy lifestyle is the use of computer-tailoring, which can be integrated into web-based programmes. Tailoring is "any combination of information or change strategies intended to reach one specific person, based on characteristics that are unique to that person, related to the outcome of interest, and have been derived from an individual assessment" (Kreuter \& Skinner, 2000, page 1; Kreuter et al., 1999a; Kreuter et al., 1999b). In computer-tailoring, personalized advice is created based on an algorithm calculated from the answers given to a questionnaire. Tailor formulas use a respondent's answers to different questions to search for relevant feedback messages from a feedback library.

A general advantage of web-based interventions - including those that use computer-tailoring - is that the programmes can be filled out anonymously, and at a time and place the individual finds convenient. Computer-tailoring is a promising strategy to stimulate health behaviour change. Earlier studies found that tailored information is perceived as more personal and relevant in comparison to general information, while tailored texts are read, saved, remembered and discussed with others more often (Brug et al., 2003; De Vries \& Brug, 1999). This strategy has been shown to be effective in behaviour change and is considered more cost-effective, since it not only leads to increased health benefits, but also to lower costs (e.g. De Vries et al., 2008a; Krebs et al., 2010; Lustria et al., 2013; Neville et al., 2009b; Ryan et al., 2002, Smit et al., 2011; Smit et al., 2013). Two meta-analyses performed by Krebs et al. (2010) and Lustria et al. (2013) regarding computer-tailored interventions for health behaviour change found small overall effect sizes. Lustria et al. (2013) found the largest effect size for nutrition behaviour interventions $(d=0.22)$ compared to smoking $(d=0.15)$, drinking $(d$ $=.08$ ) and physical activity $(d=0.06)$. Most of the previous programmes that make use of computertailoring were aimed at single behaviour change (e.g. Elfeddali et al., 2012a; Riper et al., 2009; Smit et al., 2012a; Te Poel et al., 2009; Van Stralen et al., 2011), but the trend for developing multiple behaviour change interventions is increasing (e.g. Oenema et al., 2008; Parekh et al., 2012; Vandelanotte et al., 2008; Van Genugten et al., 2012; Van Keulen et al., 2011). Krebs et al. (2010) 
found a larger effect size when intervening in two $(g=0.21)$ or three $(g=0.24)$ behaviours compared to a single behaviour ( $g=0.15$ ) or four ( $g=0.12$ ) behaviours.

\section{Theoretical framework: I-Change model}

Research has indicated that intervention programmes based on theory are the most effective (Painter et al., 2008). This dissertation uses the I-Change model (Figure 1.1) that was developed by De Vries et al. (2003). The integrated model for explaining motivational and behavioural change - shortly called the I-Change model - is the successor of the Attitude-Social influence-Self-efficacy (ASE) model (De Vries et al., 1988), and builds on other psychosocial models like the Theory of Planned Behaviour (Ajzen, 1991), the Social Cognitive Theory (Bandura, 1986), the Health Belief Model (Janz \& Becker, 1984), and the Transtheoretical Model (Prochaska \& DiClemente, 1983).

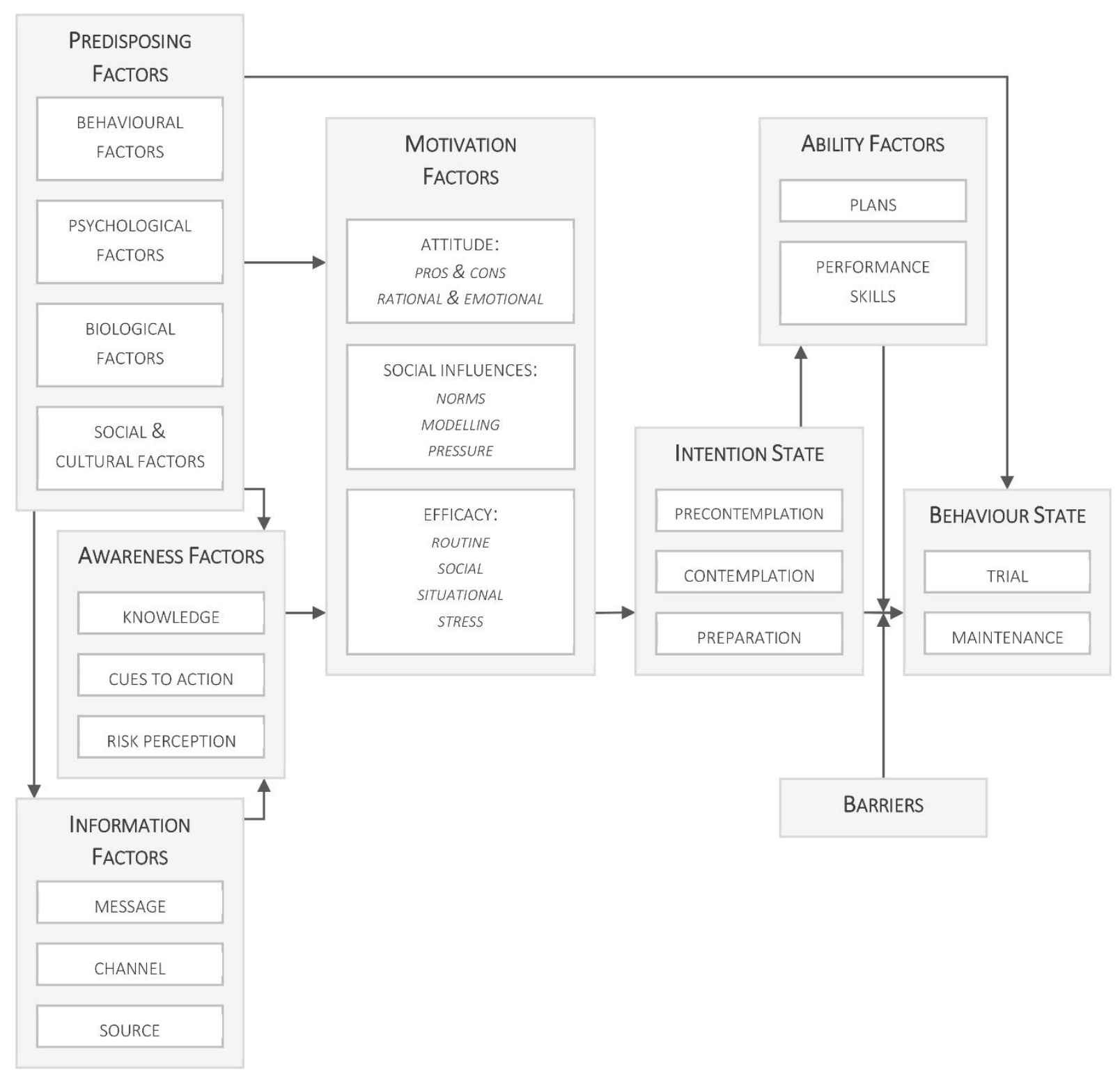

Figure 1.1 The I-Change model developed by De Vries et al. (2003; 2005) 
The I-Change model distinguishes three motivational stages, namely pre-motivational, motivational, and post-motivational. The pre-motivational stage is characterized by predisposing factors (i.e. behavioural, psychological, biological, social and cultural factors), information factors (i.e. message, channel and source) and awareness factors (i.e. knowledge, cues to action and risk perception).

The motivational factors, including attitude, social influence and self-efficacy, are influenced by the pre-motivational factors. Attitude can be subdivided into pros (i.e. perceived advantages) and cons (i.e. perceived disadvantages) of a specific behaviour. Social influence includes the factors norms (i.e. the opinion of important others in the social environment), modelling (i.e. the behaviour of important others in the social environment) and pressure (i.e. pressure or support of important others in the social environment). Self-efficacy refers to the perceived confidence of someone performing a certain behaviour.

The most proximal determinant of behaviour is the intention to perform this certain behaviour. Intention can be subdivided into different stages, such as precontemplation, contemplation and preparation and - in line with the Transtheoretical Model (Prochaska \& DiClemente, 1983) - the stages can be assessed across different time periods. The intention state is predicted by the motivational factors. If someone has the intention to perform a specific behaviour, barriers may hinder the actual performance of the behaviour: this is known as the intention-behaviour gap. A meta-analysis by Rhodes and De Bruijn (2013), for example, showed that the overall intentionbehaviour gap with regard to physical activity was $46 \%$. On the other hand, ability factors (e.g. action plans and performance skills) can help to minimize the gap and to overcome barriers. Action planning is the process of planning and performing specific actions in order to successfully change behaviour, carry out a new behaviour or maintain a current behaviour (Araújo-Soares et al., 2009; Austin \& Vancouver, 1996; Earley et al., 1987; Van Osch et al., 2009). There are two types of action planning: preparatory planning (i.e. actions which help to prepare the behaviour or behaviour change) (De Vet et al., 2011; Elfeddali et al., 2012b); and coping planning (i.e. actions to cope with difficult situations one may encounter when performing a certain behaviour) (De Vries et al., 2013; Sniehotta et al., 2006; Van Osch et al., 2010).

Different studies have confirmed the influence that different determinants have on behaviour and/or behaviour change. Various studies have tested the influence of attitude, social influence and selfefficacy on intention and behaviour. Vitória et al. (2009), for example, found that all three motivational factors are predictive of the intention to quit smoking, while Plotnikoff et al. (2013) only confirmed attitude as being a strong predictor of the intention to be physically active. A study by De Vries et al. (2013) confirmed that preparatory planning regarding smoking cessation predicts plan enactment, which, in turn, is a predictor of quitting smoking. A study by Van Osch et al. (2008) aimed at smoking cessation found support for coping planning being effective in the prevention of unwanted behaviour. Mullan et al. (2011) found in their study that respondents showing high planning ability or high inhibitory control were more likely to avoid binge drinking, and thus confirmed the added value of planning skills in interventions. 

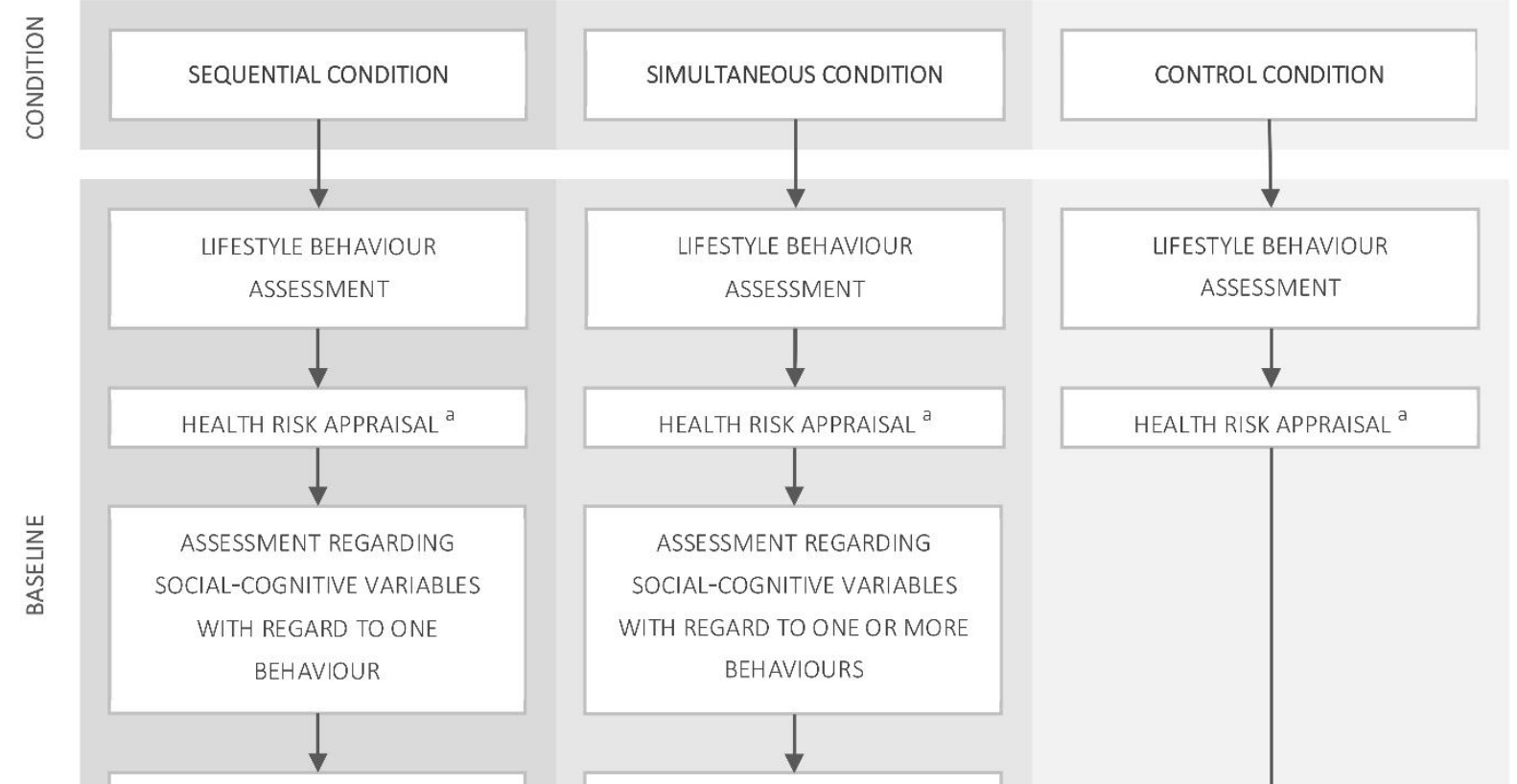

PERSONAL ADVICE ABOUT ONE UNHEALTHY BEHAVIOUR ${ }^{b}$

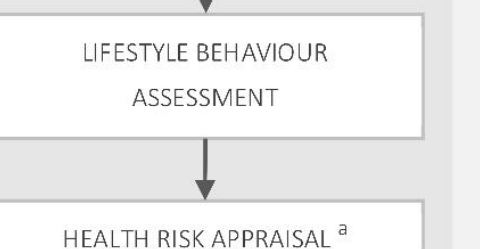

HEALTH RISK APPRAISAL ${ }^{a}$

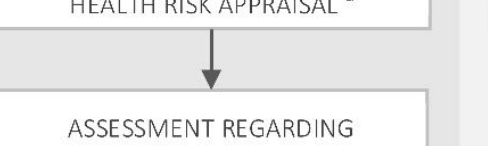

HEALTH RISK APPRAISAL SOCIAL-COGNITIVE VARIABLES

WITH REGARD TO ONE OR MORE BEHAVIOURS

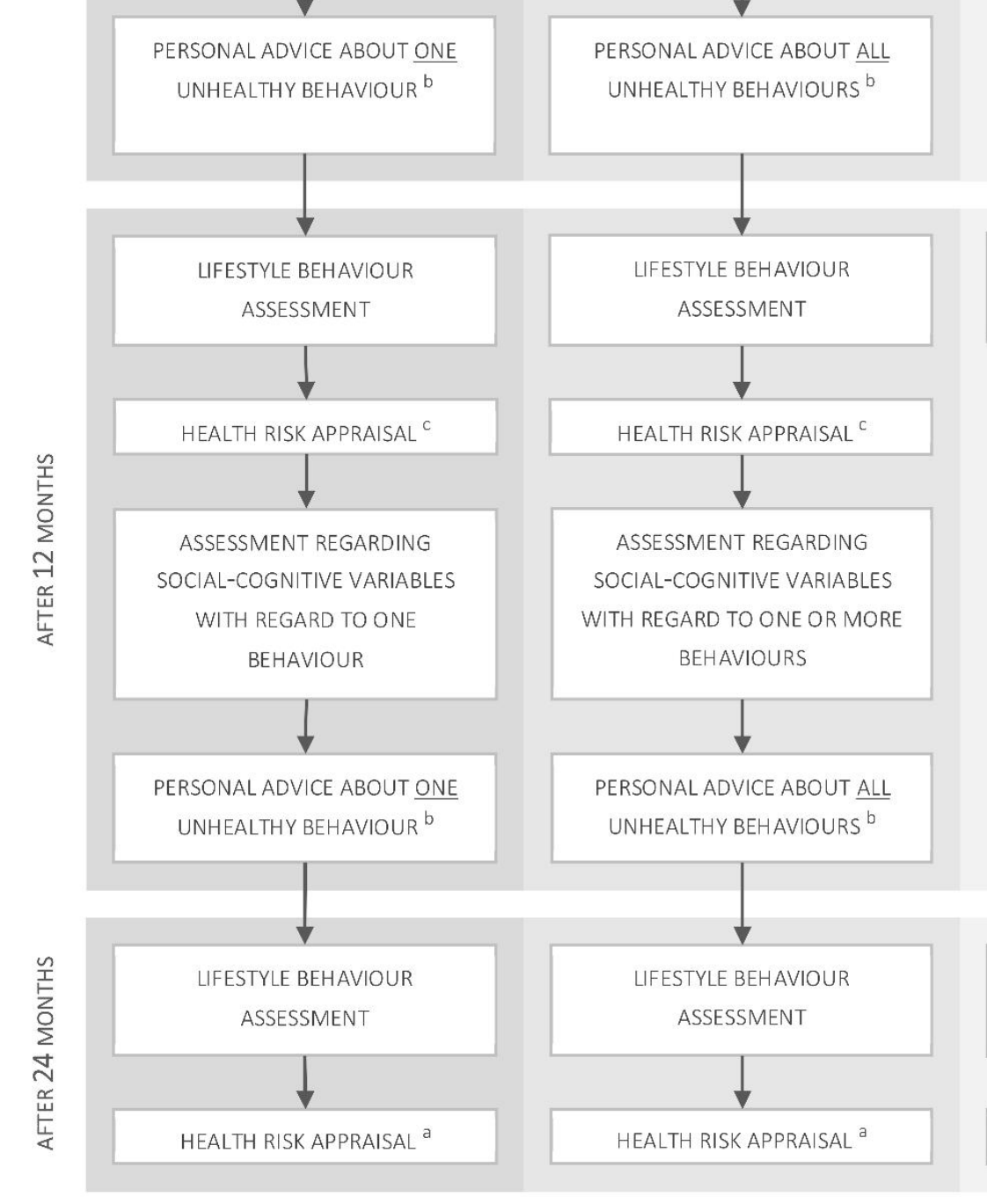

PERSONAL ADVICE ABOUT ALL UNHEALTHY BEHAVIOURS ${ }^{b}$ 


\section{Intervention programme}

The intervention programme that is described and tested in this dissertation is aimed at improving a person's lifestyle, and applies the I-Change model in the following way: after answering questions regarding the five different lifestyle behaviours, all respondents received a health risk appraisal, which gave an overview as to whether or not that individual met the various guidelines. This health risk appraisal served as a starting point to increase knowledge and to make the person aware of his or her lifestyle behaviour (pre-motivational stage). Afterwards, the experimental groups received personalized feedback regarding attitude, social influence and self-efficacy in order to motivate them to improve their lifestyle. Moreover, the concept of action planning is integrated into the programme by utilising preparatory planning and coping planning in order to prepare the individual to be able to perform the behaviour in question and to overcome potential barriers (Schulz et al., 2011). An overview of the study design is presented in Figure 1.2.

\section{Feedback messages and tailoring ingredients}

A distinction can be made between the first generation of computer-tailored interventions, which are delivered through printed materials (e.g. letters), and second generation interventions, which are delivered through interactive technology (e.g. websites or e-mails) (Norman et al., 2007). The latter have the advantage that feedback messages can be presented on the participant's screen while participating in the intervention. This means that the personalized messages are presented to the respondent immediately after answering the questionnaire. Hence, a direct interaction between the participant and the eHealth technology can be realized.

The delivery of feedback messages for multiple behaviours poses a challenge in finding a balance between completeness and conciseness. In the intervention described in this dissertation, compiling the feedback texts was executed in different ways: some feedback messages were based on the itemlevel; others on the basis of sum scores and mean scores; while other messages were created by a combination of answers given by the respondent. In this way, diversified yet also personalized feedback texts could be generated. Some examples are shown in Figure 1.3.

Different tailoring ingredients can be adopted in the tailored messages: using personalization (e.g. the name of the respondent); giving normative and ipsative feedback (i.e. comparing current scores to previous scores); repeating the respondent's answers; confirming and increasing knowledge; correcting wrong assumptions; providing tips/suggestions; giving positive reinforcement; showing sympathy; making them aware of the behaviour of others (awareness raising); and motivating, convincing and stimulating them to think about some (new) information (Dijkstra, 2005; Dijkstra \& De Vries, 1999). Moreover, graphics and pictures can be used as aids to visualize some information. A study by Dijkstra (2005) showed that, in terms of smoking cessations, personalization and feedback are working mechanisms that lead to more health behaviour changes compared to non-tailored material. In addition, tailored interventions have proven to be more effective when using multiple 
tailoring moments compared to a single feedback moment (Brug et al., 1998; Dijkstra et al., 1998c; Elfeddali et al., 2012a).

TAILORING APPLICATION

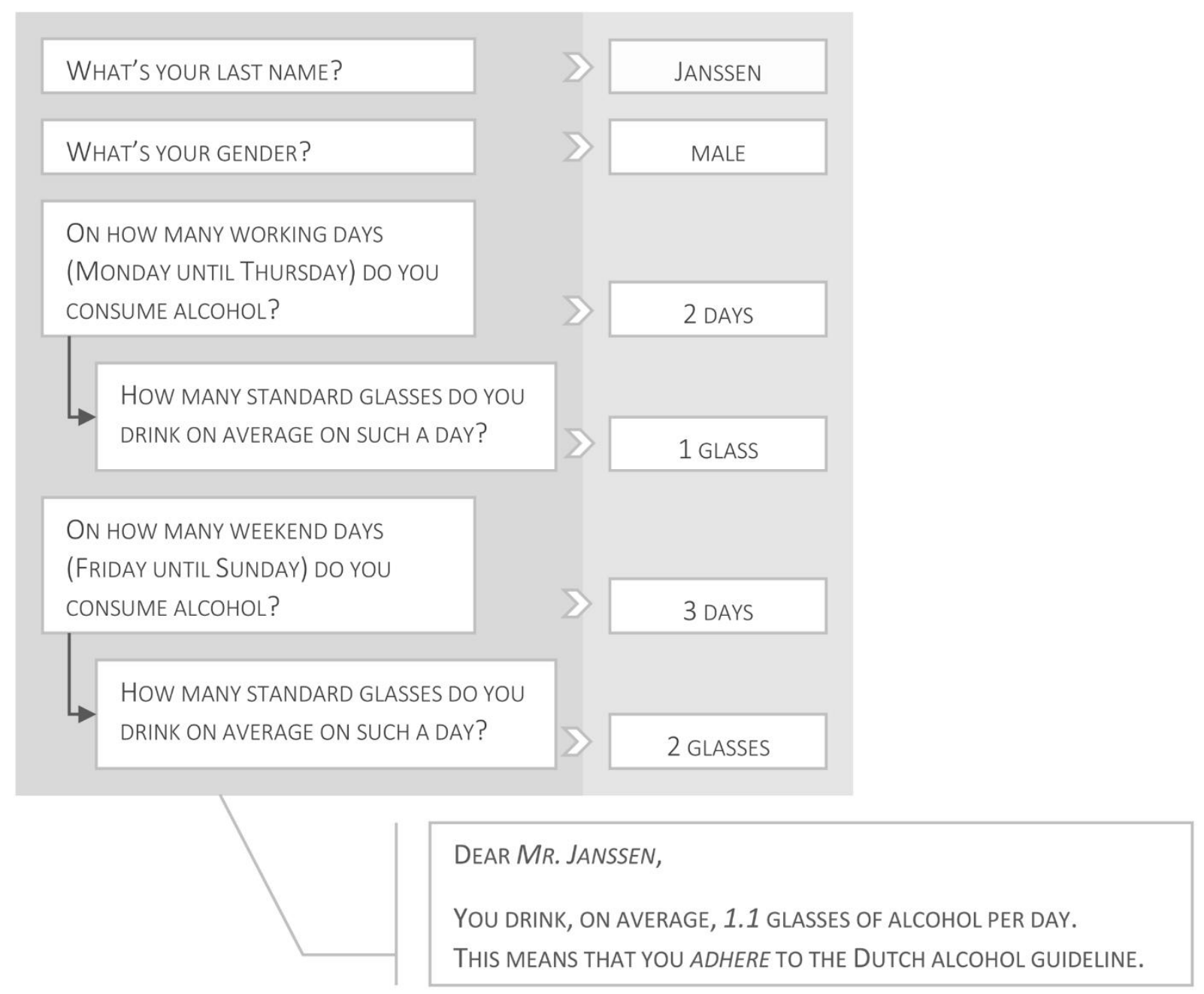

Figure 1.3 Example of composing a tailored feedback message

The application of theory has been proven to raise the effectiveness of a web-based intervention. Webb et al. (2010) found that interventions that make extensive use of theory, especially the Theory of Planned Behaviour (Aijzen, 1991), are more effective in reaching health behaviour change than interventions that made less extensive or no use of theory. The I-Change model was used in our intervention to target the different theoretical constructs integrated in the model. In addition, the study by Webb et al. (2010) identified behaviour change techniques as successful ingredients for health behaviour change. The most effective techniques were the provision of stress management, general communication skills training, modelling, relapse prevention and coping planning, facilitating social comparison, goal setting, action planning, and the provision of feedback on performance. In our intervention programme, different health behaviour change techniques were combined and integrated. 


\section{Sequential versus simultaneous tailoring}

According to Prochaska et al. (2008, page 183), multiple health behaviour change interventions are defined as "efforts to promote two or more health behaviours". Changing lifestyle behaviours is complex and challenging, and according to Vandelanotte (2013), the optimal design approach is not yet known. In this dissertation, two different strategies were tested: a sequential behaviour change strategy in which personal advice on multiple lifestyle behaviours is provided consecutively at different points in time; and a simultaneous behaviour change strategy in which personal advice on multiple lifestyle behaviours is given at the same time. Results regarding the effectiveness are to some extent controversial, meaning that more research is needed (Hyman et al., 2007; King et al., 2013; Vandelanotte et al., 2005; Vandelanotte et al., 2007a; Vandelanotte et al., 2008).

Focus on one behaviour at a time may have the advantage that an intervention is shorter and/or can give more detailed information on one specific behaviour; on the other hand, respondents may perceive themselves as having a low level of autonomy because they have to limit themselves to a single behaviour at the beginning.

Intervening on several modifiable risk behaviours simultaneously means that respondents may receive a lot of information, and will be confronted with more options and choices. Consequently, the length of such an intervention increases, as well as the time and cognitive effort needed to process the information provided. As such, high demands are put on the participant. It could be that people become overwhelmed by the amount of information and the complexity of the programme, which could lead to fewer energy resources to change, a reduction in a person's motivation and energy also known as ego depletion (Baumeister et al., 1998; Baumeister et al., 2003) - and a more negative evaluation of the intervention. On the other hand, direct exposure to multiple behaviours at the beginning of an intervention could have advantages for those people who drop out early (King et al., 2013). In this method, participants have already received more information during their first visit compared to those of a single behaviour intervention whereby those who drop out after their first visit only receive information about a single behaviour.

The probability that a respondent will engage in changing a second behaviour may increase when an individual has successfully changed a first behaviour (King et al., 1996). This means that change may occur in another behaviour without having to directly intervene on it. For example, an increase in physical activity may lead to improved eating habits (Mata et al., 2009). One explanation could be that different behaviours often share the same determinants. If someone changes his or her attitude regarding a healthy diet, for example, this person may also change the attitude regarding regular physical activity (Noar et al., 2008). Once effort exerted, one may also wish to reach their goal to a higher extent (e.g. weight loss). Moreover, self-efficacy and motivation to successfully perform a certain behaviour often increases after successfully changing one behaviour (Center for Substance Abuse Treatment, 1999; King et al., 2013). This implies that a person is more likely to change a second behaviour after a successful change due to more self-confidence in changing behaviour and higher motivational levels. Such transfer, synergistic or spillover effects may occur in both tailoring strategies (Kremers et al., 2004), but it is unclear in which strategy such effects are strongest. 
Other studies found that the optimal strategy possibly depends on the person in question's readiness to change. An approach aimed at simultaneous multiple behaviours could work better for individuals who see changes in different behaviours as part of a larger approach to a healthier lifestyle (Koshy et al., 2012). On the other hand, a single or sequential approach could work better for unmotivated individuals, since they can initially focus on improving a behaviour that is easier to adopt, whereas targeting multiple behaviours at one time may lead to decision fatigue or ego depletion, especially among less motivated people (Hagger et al., 2010; Muraven \& Slessareva, 2003).

\section{Alternating versus summative tailoring}

Prior research has shown that completion rates are low in Internet interventions (e.g. Postel et al., 2011b; Powell et al., 2012). To gain more insight into the nature of dropout, an additional study was included in this dissertation in order to examine the difference in completion and re-visits among a study group that received one of two different versions of a web-based tailored alcohol intervention: alternating versus summative. The difference between these versions will be explained below.

In the first version, one experimental group received questions and personal advice alternately (alternating condition), which means that the feedback message was divided into a series of messages discussing individual topics. These messages were offered while the respondent was still completing the web-based session. In the second version, the other experimental group received a complete set of personal advice after having answered all the questions (summative condition). This means that a single set of feedback messages was provided in one go at the end of the web-based session. Both strategies could be used to hold respondents' attention in online interventions. On the one hand, one could argue that the attrition rate within the alternating condition would be lower because respondents are rewarded while filling in the questionnaires, and are thereby motivated to continue. On the other hand, the attrition may be lower in the summative condition because respondents have to wait until the end of the questionnaire before receiving their requested tailored feedback. In this dissertation, the dropout rate, effectiveness and user satisfaction of these two kinds of feedback strategies are compared.

\section{Participation, completion and re-visits}

Web-based lifestyle interventions suffer from high dropout rates. Earlier research has shown that intervention effects are likely to be stronger among respondents with higher levels of website use (Tate et al., 2001). However, before stimulating engagement in the intervention, the reach of participants is a prerequisite.

This intervention was disseminated in collaboration with different Regional Health Authorities (RHAs) in two provinces of the Netherlands: North-Brabant and Zeeland. Every four years, the RHAs invite a representative segment of adults living in the Netherlands to fill out a questionnaire: the so-called 
Adult Health Monitor. This questionnaire is sent as a written version to the population. In the invitation letter, information about the web-based version of the questionnaire is introduced. Individuals who decide to fill out the electronic version are invited to receive a health risk appraisal based on their answers to the Adult Health Monitor questions. The programme at hand was integrated into the Monitor so that the RHAs could extend and improve their health education mission by offering respondents tailored feedback regarding lifestyle behaviours. Furthermore, the health risk appraisal was intended to be an extra motivation for people to participate in the study, and it was hoped that we could achieve an optimal dissemination of our intervention due to a total reach of approximately 100,000 inhabitants via the monitoring tool (Schneider et al., 2011; Schulz et al., 2011). In this dissertation, we have studied whether this dissemination strategy via the RHAs was successful in terms of reach, completion and re-visits.

\section{Economic evaluation}

On the one hand, stimulating a healthy lifestyle is an important method of improving health and quality of life, and preventing illness; it is also important as a means of reducing health care costs and the costs of lost productivity that are associated with chronic illnesses (Rappange et al., 2009; Von Lengerke \& Krauth, 2011). Moreover, diminished quality of life is related to diseases and illnesses (Davies et al., 2012b). Hence, besides studying the effectiveness of an intervention in terms of lifestyle behaviour change, it is also important to investigate the cost-effectiveness and cost-utility of an intervention. Cost-effectiveness analyses are used to estimate the ratio between costs and outcomes (e.g. effects regarding lifestyle change) of an intervention; cost-utility analyses estimate the ratio between costs and benefits in terms of the number of gained years living at full health (i.e. Quality Adjusted Life Years; QALYS). Due to budget cuts and limited funds for health promotion programmes in the Netherlands (and many other countries), economic evaluations are of great importance for making evidence-based decisions regarding large-scale implementation of (computer-tailored) interventions (National Government, 2011; Tate et al., 2009). In a time of scarce resources, decisionmakers at the policy level need to be informed about the costs and effects of an intervention, so that they are better able to select the most cost-effective alternative for potential large-scale implementation. Internet-based measures have an advantage over telephone or face-to-face counselling interventions in that they involve low intervention costs. Moreover, the reach of Internetbased interventions is high, which is likely to increase their cost-effectiveness. As web-based lifestyle interventions promise to stimulate healthy behaviours, respondents are likely to lower their risk of developing chronic illnesses which, in turn, is related to lower health care costs.

The aim of an economic evaluation is to relate the costs to the effects of the intervention, and to compare the results with the costs and effects of the best alternative intervention or care as usual (Drummond et al., 2005). It is recommended that an economic evaluation should be performed from the societal perspective, since this perspective allows the assembly of optimal societal decisions (Jönssen, 2009). This implies that intervention costs, health care costs, patient/respondent costs and productivity costs are assessed, as well as effects in terms of QALYs, quality of life and lifestyle change. 
Finally, an economic evaluation aims to identify those interventions that achieve the most population health benefits relative to the required costs (Cobiac et al., 2009). We performed a trial-based economic evaluation from a societal perspective, according to the methods set out in the Dutch guidelines for cost analyses (Hakkaart-Van Roijen et al., 2010) and pharmaco-economic research (Health Care Insurance Board, 2006).

\section{AIM OF THE DISSERTATION}

The aim of this dissertation is fourfold: (1) to describe the development process of a web-based tailored multiple behaviour change intervention; (2) to study the adoption and dissemination of the programme through different RHAs (Dutch: GGD); (3) to analyse the use of the programme by respondents; and (4) to evaluate the programme through a study of its effectiveness, as well as the cost-effectiveness and cost-utility of the intervention programme.

\section{RESEARCH QUESTIONS}

The following two questions will be addressed in this dissertation: (1) What is the potential of a tailored multiple behaviour change intervention in terms of first use and re-use, behavioural change, appreciation and cost-effectiveness?; and (2) Which tailoring strategies should be used in a tailored multiple behaviour change intervention?

\section{OUTLINE OF THIS DISSERTATION}

In chapter 2, the intervention development is described in a study protocol, with particular focus on a multiple behaviour change intervention for adults.

In chapter 3, the dissemination of the intervention via different RHAs in the Netherlands is described. An exploration was carried out to investigate the characteristics of people who were interested in such a tailored programme, as well as those who actually took part in the study.

In chapter 4, the first visit use is presented. This chapter addresses participants' characteristics associated with completion and the dropout rates of the intervention. Furthermore, differences in dropout rates between a sequential and a simultaneous approach were examined.

In chapter 5, we examined which lifestyle sub-modules of the programme (i.e. physical activity, vegetable consumption, fruit consumption, alcohol intake and smoking) were respondents' preferred choices to concentrate/work on. 
In chapter 6, a separate study is performed regarding the effectiveness of the alcohol module in terms of alcohol reduction among adults living in Germany. In this study, two different tailoring strategies were compared, namely an alternating and a summative strategy.

In chapter 7, the multiple behaviour change intervention is evaluated. The effectiveness in terms of behavioural change is discussed, as well as the process evaluation executed among the respondents. Differences between a sequential and a simultaneous tailoring strategy are studied.

In chapter 8 , the cost-effectiveness and cost-utility of multiple behaviour change interventions are discussed.

Finally, in chapter 9, the main findings of the current dissertation are summarized and discussed. Theoretical as well as practical implications of the different studies are given, and directions for further research are outlined. 



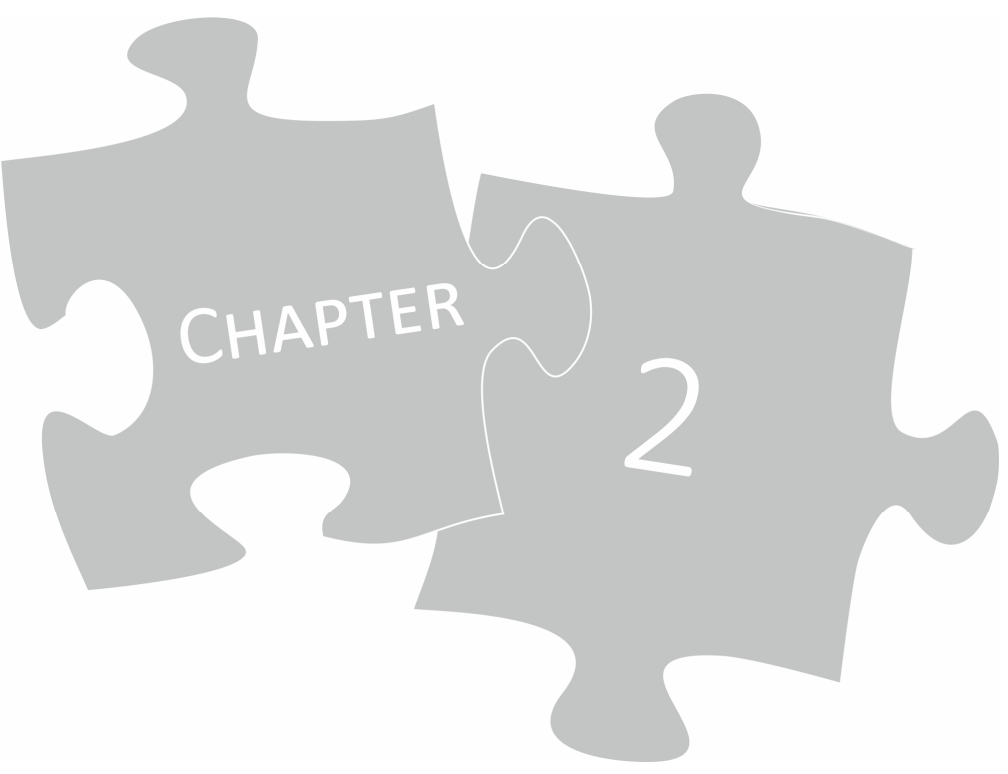

TESTING A DUTCH WEB-BASED TAILORED LIFESTYLE PROGRAMME AMONG ADULTS: A STUDY PROTOCOL

This chapter has been published as:

Schulz DN, Kremers SP, van Osch LA, Schneider F, van Adrichem MJ, de Vries H. Testing a Dutch webbased tailored lifestyle programme among adults: a study protocol. BMC Public Health 2011; 11:108. 


\section{ABSTRACT}

Background: Smoking, high alcohol consumption, unhealthy eating habits and physical inactivity often lead to (chronic) diseases, such as cardiovascular diseases and cancer. Tailored online interventions have been proven to be effective in changing health behaviours. The aim of this study is to test and compare the effectiveness of two different tailoring strategies for changing lifestyle compared to a control group using a multiple health behaviour web-based approach.

Methods: In our Internet-based tailored programme, the five lifestyle behaviours of smoking, alcohol intake, fruit consumption, vegetable consumption, and physical activity are addressed. This randomized controlled trial, conducted among Dutch adults, includes two experimental groups (i.e. a sequential behaviour tailoring condition and a simultaneous behaviour tailoring condition) and a control group. People in the sequential behaviour tailoring condition obtain feedback on whether their lifestyle behaviours meet the Dutch recommendations. Using a step-by-step approach, they are stimulated to continue with a computer-tailored module to change only one unhealthy behaviour first. In the course of the study, they can proceed to change a second behaviour. People in the simultaneous behaviour tailoring condition receive computer-tailored feedback about all their unhealthy behaviours during their first visit as a stimulation to change all unhealthy behaviours. The experimental groups can re-visit the website and can then receive ipsative feedback (i.e. current scores are compared to previous scores in order to give feedback about potential changes). The (difference in) effectiveness of the different versions of the programme will be tested and compared to a control group, in which respondents only receive a short health risk appraisal. Programme evaluations will assess satisfaction with and appreciation and personal relevance of the intervention among the respondents. Finally, potential subgroup differences pertaining to gender, age and socioeconomic status regarding the behaviour effects and programme evaluation will be assessed.

Discussion: Research regarding multiple behaviour change is in its infancy. We study how to offer multiple behaviour change interventions optimally. Using these results could strengthen the effectiveness of web-based computer-tailored lifestyle programmes. This study will yield new results about the need for differential lifestyle approaches using Internet-based expert systems and potential differences in subgroups concerning the effectiveness and appreciation. 


\section{BACKGROUND}

Cardiovascular diseases (CVD) and cancer are the two major causes of illness and death in the Netherlands as well as in other Western countries. Causal factors of these diseases are partly related to unfavourable lifestyle behaviours, such as smoking, high alcohol consumption, unhealthy eating habits and physical inactivity (National Institute for Public Health and the Environment, 2002; World Health Organization, 2007). In the Netherlands, public health guidelines are defined for these health behaviours, i.e. non-smoking, not drinking more than one (women) or two (men) glasses of alcohol a day (Netherlands Institute of Mental Health and Addiction, 2013a), eating two pieces of fruit per day (The Health Council of the Netherlands, 2006), eating 200 grams of vegetables per day (The Health Council of the Netherlands, 2006), and being moderately physically active for 30 minutes at least five days a week (Kemper et al., 2000). Approximately three quarters of the Dutch population eats too little fruit and vegetables, nearly half of the population does not meet the recommendation for physical activity, nearly one third smokes and it is estimated that a comparable percentage of people drinks too much alcohol (De Vries et al., 2008c; Statistics Netherlands, 2007a). No single behaviour can be ascribed as a sole causal factor in the development of diseases such as CVD or cancer. Recent research shows that about $5 \%$ to $10 \%$ of the population complies with all these public health guidelines and that $5 \%$ to $10 \%$ does not comply with any of the five guidelines (De Vries et al., 2008b).

Interventions are needed in order to encourage people to adopt a healthier lifestyle. Nowadays, 92.9\% of the Dutch population have access to the Internet (Internet World Stats, 2012). This implies that web-based lifestyle interventions have the potential to reach a large number of people (Cobiac et al., 2009; Statistics Netherlands, 2013a). Web-based computer-tailoring is a relatively new effective technique for health education. Computer-tailoring is any combination of information or change strategies intended to reach one specific person, based on characteristics that are unique to that person, related to the outcome of interest, and have been derived from an individual assessment (Kreuter et al., 1999a; Kreuter et al., 1999b). Positive aspects of web-based computer-tailored programmes are that these can be applied in privacy, for example at home, and at a time the respondent finds convenient (Riper et al., 2008), and that they can be integrated in other interventions (De Vries \& Brug, 1999). A computer-tailored programme even has some key benefits in comparison to non-tailored materials. It can provide personalized feedback which attracts attention and consists of less unnecessary information than general material (Kreuter et al., 2000b). Tailored messages are usually better read, saved, remembered and discussed with others than non-tailored materials (Campbell et al., 1994; De Vries \& Brug, 1999; Smeets, 2006). Computer-tailoring is costeffective due to the fact that it can be provided via the Internet and can reach many people (Brug et al., 2003; De Vries \& Brug, 1999). Computer-tailoring seems to be more effective in comparison to general messages about health beliefs and health behaviour (Brug et al., 1999a; Campbell et al., 1994; De Vries \& Brug, 1999) as shown by their effects on increasing smoking cessation (Dijkstra et al., 1998b), decreasing alcohol consumption (Netherlands Institute for Health Promotion and Disease Prevention, 2009; Riper et al., 2008), increasing fruit and vegetable intake (Baker \& Wardle, 2002; Brug et al., 1998; Irvine et al., 2004; Oenema et al., 2005) and increasing physical exercise (Campbell et al., 2004; Marcus et al., 1998; Smeets et al., 2008; Spittaels et al., 2007b). In the past, most 
computer-tailored programmes addressed only one behaviour. By now, the focus on multiple behaviours has become increasingly popular (Brug et al., 1998; De Vries et al., 2008a; Jacobs et al., 2004; Oenema et al., 2008; Smeets et al., 2007). However, the best strategy for offering health information to motivate the population to change multiple behaviours has not yet been determined.

Two different types of strategies can be distinguished in this respect: a simultaneous behaviour change strategy in which personal advice on multiple lifestyle behaviours is given at the same time; and a sequential behaviour change strategy in which personal advice on multiple lifestyle behaviours is provided consecutively at different points in time. Both strategies have potential advantages as well as disadvantages which may have influence on the effectiveness and appreciation of the tailored programme. In the simultaneous behaviour change strategy, people receive a lot of information and they may be inclined to choose one behaviour from the range of options and to autonomously change or add to this choice at any time. However, people in the sequential behaviour change condition may perceive a lower level of autonomy since they have to limit themselves to one single behaviour at first (Markland \& Vansteenkiste, 2007; Zuckerman et al., 1978). Due to the amount of information that is given in the simultaneous behaviour change strategy, participants actually receive more options and choices and this strategy may possibly be more effective for people who are already motivated to change their lifestyle. On the other hand, it is conceivable that individuals in this situation become overwhelmed due to the amount of information, leading to too few energy resources to change (Baumeister et al., 1998) and a more negative feeling regarding the programme. This may be particularly true for people who are not ready to make changes in their lifestyle.

Recent research suggests that the probability of enhancing a second behaviour increases when an individual has successfully changed a first behaviour (King et al., 1996). This means that, for example, an increase in physical activity may lead to improved eating habits (Mata et al., 2009). Such synergistic or spill-over effects may occur in both strategies (Kremers et al., 2004), but it is unclear in which strategy such effects are strongest.

The tailored programme used in the current study is theoretically based on the integrated model for explaining motivational and behavioural change (the I-Change model) (De Vries et al., 2003). The IChange model builds on the Attitude-Social influence-Self-efficacy (ASE) model (De Vries et al., 1988), which in turn integrates ideas from social-cognitive models, such as the Theory of Planned Behaviour (Aijzen, 1991), the Social Cognitive Theory (Bandura, 1986), the Health Belief Model (Janz \& Becker, 1984), and the Transtheoretical Model (Prochaska \& DiClemente, 1983). According to the I-Change model, the most proximal determinant of behaviour is the intention to perform this certain behaviour. If someone has the intention to perform a specific behaviour, barriers may hinder the actual performance of the behaviour. On the other hand, ability factors (i.e. action plans, goal action) can help to overcome these barriers. Intention is determined by the motivational factors attitude, social influence and self-efficacy, which in turn are influenced by awareness factors (i.e. knowledge, cues to action, risk perception), predisposing factors (i.e. behavioural, psychological, biological, social and cultural factors) and information factors (i.e. message, channel, source). 
The main goal of this study is to test and analyse the effects of two different strategies (i.e. sequential and simultaneous behaviour change feedback) using web-based computer-tailored technology. The effects will be compared with those of a control group. Furthermore, we will conduct a programme evaluation and explore potential differences in effectiveness and appreciation of the intervention among subgroups (i.e. socioeconomic status- (SES), age- and gender differences) in order to optimize the programme so that it fits better to the individuals' characteristics, needs and preferences.

\section{METHODS}

\section{Design}

The current study is related to a larger study, which was approved by the Medical Ethics Committee of Maastricht University and the University Hospital Maastricht (NL27235.068.09/MEC 09-3-016). In that study, we assess level of adoption of the computer-tailored programme by focusing on three different exposure rates: first use of the intervention, prolonged use (staying on the intervention for a substantial period of time) and sustained use (re-visits to the intervention). Besides this, satisfaction with the service will be carried out.

The present study, which is registered at the Dutch Trial Register (NTR2168), consists of a randomized controlled trial (RCT) including two experimental groups, namely a sequential behaviour tailoring condition (SeqC) and a simultaneous behaviour tailoring condition ( $\mathrm{SimC}$ ), and a control group. The conditions will be described in detail below. A design with a baseline measurement and two follow-up measurements at 12 and 24 months is used.

\section{Participants, inclusion criteria and procedure}

The study is part of an initiative of different Dutch Regional Health Authorities (RHAs) of the provinces North-Brabant and Zeeland. Our computer-tailored programme is partly integrated in the Adult Health Monitor 2009 which is conducted by the RHAs. With this monitoring instrument, the health status of people living in the Netherlands is assessed. Every four years, a representative sample of the population aged between 18 and 65 years is recruited in order to fill out this questionnaire. It contains the following topics: demographics; aspects of general health (e.g. physical health, mental health, social health, lifestyle behaviours); and health-related topics (e.g. social and physical environment) (National Institute for Public Health and the Environment, 2005). As part of an additional service, participants completing this Monitor online subsequently obtain the opportunity to receive tailored feedback about their health behaviour via the new computer-tailored programme. Therefore, some data the person fills out during the Adult Health Monitor (e.g. demographics and data regarding the five lifestyle behaviours: smoking, alcohol intake, fruit consumption, vegetable consumption and physical activity) are copied to the computer-tailored programme. Before this, participants receive information about the purpose of the current study and an explanation of the use/handling of their 
data. We ask for written informed consent for their participation in the project. Confidentiality is ensured and it is explained to respondents that they could withdraw participation at any moment.

Approximately three weeks after completing the Monitor, participants who are interested in obtaining tailored feedback about their health behaviour receive an e-mail including an invitation to log in to the computer-tailored programme by use of their personal login code and password. After successfully logging in, respondents receive the health risk appraisal (i.e. feedback concerning their lifestyle and information about whether they meet the public health guidelines) based on their answers to the Adult Health Monitor, and the experimental groups receive some additional questions (i.e. attitude, social influence, preparatory action plans, self-efficacy, coping plans) and personal advice. The different parts of the programme will be described in detail below.

\section{The web-based computer-tailored programme}

To deliver personalized advice, three inter-related elements are necessary: a screening instrument to measure demographics, health status, health behaviour and behavioural determinants; a message source file containing all tailoring messages; and a computer programme that offers the possibility to analyse the screening results and to select the correct messages from the message file (De Vries \& Brug, 1999; Dijkstra \& De Vries, 1999).

The intervention programme is developed by means of the "TailorBuilder"-software (OverNite Software Europe B.V., The Netherlands). The TailorBuilder is a web-based instrument that was specifically developed to create and conduct online questionnaires and design and implement tailored advice. Respondents can login via a website on which general information about smoking, alcohol intake, fruit and vegetable consumption, and physical activity is given as well as detailed information about the study, frequently asked questions and a contact form.

The web-based computer-tailored programme combines several programmes which have already been proven to be effective in increasing smoking cessation (Dijkstra et al., 1998b), reducing the consumption of alcohol (Riper et al., 2008), promoting the intake of fruit and vegetables (Oenema et al., 2005), and increasing the level of physical activity (Smeets et al., 2008). Tailoring algorithms that combine the responses of a person on specific questions with concurrent feedback messages generate highly personalized advice to the respondent which they receive on their screen and that can also be printed. The following two paragraphs describe the questionnaire and the advice components in more detail. 


\section{Measurement instruments}

\section{Demographic information}

The following demographic variables are measured: age, gender, height, weight, marital status, religious background, ethnicity, educational level, current work status, and income.

\section{Health status}

Respondents are asked if they suffer from high blood pressure and different kinds of diseases, such as diabetes and cancer. To measure quality of life, the SF-12 Health Survey (Ware et al., 1995, 1996) and the Mental Health Inventory (MHI-5) (Perenboom et al., 2000) are used. To assess symptoms of depression and anxiety, the Kessler Psychological Distress Scale (K10) (Kessler \& Mroczek, 1994) is used.

\section{Lifestyle behaviours}

Smoking is measured by the abbreviated version of the Fagerström Test for Nicotine Dependence (Heatherton et al., 1991). Alcohol consumption is measured by the Dutch Quantity-FrequencyVariability (QFV) questionnaire (Lemmens et al., 1992). Fruit and vegetable intake is measured using Food Frequency Questionnaires (FFQ) for fruit and vegetable intake (National Institute for Public Health and the Environment, 2005). Physical activity is measured by the Short QUestionnaire to ASsess Health-enhancing physical activity (SQUASH) (Wendel-Vos et al., 2003).

\section{Intention}

The intention to change behaviour is measured by one item. This item is an extended version of the 'stages of change' concept (Prochaska \& DiClemente, 1983; Prochaska et al., 1997) using an algorithm consisting of ten stages varying from unawareness to maintenance.

\section{Attitude}

To measure attitude concerning the different health behaviours, participants have to indicate on a five-point scale to what extent they 'totally disagree' or 'totally agree' with a total of six statements. Pros (i.e. advantages) of the health behaviour under consideration are assessed by three items, such as "Regular physical activity is good for my health". Cons (i.e. disadvantages) are assessed by another three items, such as "Regular physical activity costs a lot of time". 


\section{Social influence}

Social influence is measured by three different concepts, namely social norms, social modelling and social support. Social norms are assessed by one statement regarding the opinion of the direct environment (i.e. partner, family members, friends and colleagues) concerning each health behaviour. Respondents are asked to complete statements, such as "According to the people within my direct environment...", by choosing one of five options ranging from "I certainly should not smoke" to "I certainly should smoke". Social modelling is assessed by asking the participants how many people of their direct environment engage in the healthy behaviour, e.g. eating at least two pieces of fruit every day. A five-point scale is used, ranging from 'everybody' to 'nobody'. Social support is measured by one item assessing the degree of support respondents receive from their direct environment to engage in the healthy behaviour, on a four-point scale, ranging from 'yes, they support/stimulate me a lot' to 'no, they do not support/stimulate me at all'.

\section{Preparatory plans}

Preparatory plans are measured by three items for each health behaviour, such as "I intend to become a member of a sports club". Respondents have to specify whether they have the intention to use a specific preparatory plan in order to adopt the particular healthy lifestyle. A five-point scale is used, ranging from 'yes, definitely' to 'no, definitely not'.

\section{Self-efficacy}

Self-efficacy is measured by means of six items. Participants have to indicate on a five-point scale to what extent they feel able/unable to engage in or refrain from the key health behaviour when encountering difficult situations (i.e. situations that might lead to the performance of the unhealthy behaviour), e.g. "I am able to drink not more than two glasses of alcohol when I am at a party".

\section{Coping plans}

Coping plans are assessed by asking participants to indicate on a five-point scale to what extent they agree/disagree with a total of six statements. Every statement deals with a specific plan regarding how to cope with difficult situations, e.g. "I have made a plan to be sufficiently physically active even when I have a great deal to do". These questions are based on the difficult situations used for the selfefficacy items. 


\section{Feedback procedure}

The message source file generates the tailored pieces of advice about the health behaviours, attitude, social influence, preparatory plans, self-efficacy and coping plans. Hence, respondents receive tailored pieces of advice on their computer screen immediately after completion of each part (concept) of the questionnaire in order to avoid a very long text at the end of the programme. Either text-based messages or graphic feedback are given, in addition to pictures. Besides, in some pieces of advice, links to detailed information are added for people who want to receive more information about certain topics. Tailor formulas are used in order to determine which advice the respondent receives. After completion of the programme, respondents receive an overview of all the pieces of advice they have received while doing the test. This total advice can be printed and is also sent to the respondent's e-mail address, so that it can be re-read at any time. The programme consists of two kinds of feedback, namely (1) a health risk appraisal and (2) personal advice on psychosocial constructs.

\section{Health risk appraisal}

The first part of the feedback consists of a health risk appraisal which comprises information about the Dutch guidelines defined for the health behaviours. Respondents obtain feedback via text that is illustrated for each behaviour with a green, orange or red traffic light to indicate the level of adherence to the Dutch guideline (Bergström et al., 2008). A green light implies adherence to the guideline; an orange light implies non-adherence by being close to the healthy level whereas a red light represents non-adherence. Respondents receive an explanation regarding their score for each behaviour. For instance, "You drink approximately five glasses of alcohol per day. This means that you do not meet the Dutch guidelines defined for acceptable alcohol consumption." Furthermore, respondents can click on links to receive additional and more detailed information about the guidelines and the specific health behaviour. For example, they can receive information about the relation between alcohol intake and pregnancy and the related danger for the unborn child. Furthermore, respondents' scores are depicted graphically via a bar chart that compares the respondents' behaviour (e.g. the number of alcoholic drinks a day) with the guideline for this behaviour. At the end of the health risk appraisal, respondents receive an overview illustrating the lifestyle behaviour status in terms of five traffic lights (i.e. one traffic light per health behaviour) side by side so that they can evaluate their lifestyle at a glance. 


\section{Personal advice}

In the second part of the programme, a progressive scheme of four steps is applied.

Step 1 During the first step, emphasis is placed on attitude. The advantages and disadvantages of the relevant behaviour are explained, and applied to the beliefs of the respondent. In other words, knowledge is confirmed or corrected, and (new) information is given and deepened.

Step 2 During the second step, we focus on discussing social influences and explain to the respondent that the social environment may influence the behaviour of the respondent and the other way around. They receive information about how to cope with pressure from others, e.g. how to refuse a cigarette. The importance of social support and of being a good role model is explained. In addition, the possibility of changing the unhealthy behaviour with the aid of others (e.g. increasing the amount of physical activity by taking exercise together with a friend) is also mentioned.

Step 3 During the third step, the concept preparatory action plans is addressed. Respondents are invited to make action plans in order to prepare their behavioural change. For instance, we recommend that respondents take fruit along when they go somewhere (e.g. to work) to guarantee availability at the moment they want it. When they have indicated their specific plans, they receive positive reinforcement if they have made preparatory plans and additional tips on how to increase the success of their plan. If they indicate that they are not willing to carry out a plan, we try to persuade the person to try out an alternative plan and provide advice and suggestions on how to do so.

Step 4 During the fourth step, attention is given to the concepts self-efficacy and coping plans. A summary is given relating to the situations the respondents indicate that they expect to be difficult and for which difficult situations the respondent has or has not already made a plan. For example, if someone reports drinking alcohol when he or she feels depressed, it is explained that alcohol does not help against negative feelings in the long run and that it would be better to do something other than drinking alcohol in such a situation, for instance, to go out for a walk or to talk about the feelings with a friend. Respondents receive an overview of their perceived difficult situations and their plans in order to facilitate remembering these. If people perceive a situation as difficult, but they have made no plan, we give a number of specific tips on how to behave in a healthy way in this particular situation.

\section{The two experimental groups and the control group}

As presented in Figure 2.1, the three groups differ in the amount of feedback they receive. The experimental groups have the option to obtain health risk appraisals and personal advice as often as they want whereas the control condition only receives the health risk appraisal once. Every time the experimental groups visit the programme, ipsative feedback is given about their health behaviour. This implies that the health behaviour scores of the current visit are compared to the scores of the last visit; feedback is given about a potential change. Furthermore, all scores are saved and illustrated in a 
graph in order to give the respondent the option to monitor the total change process at a glance. Ipsative feedback is not available for the control group.

CONDITION

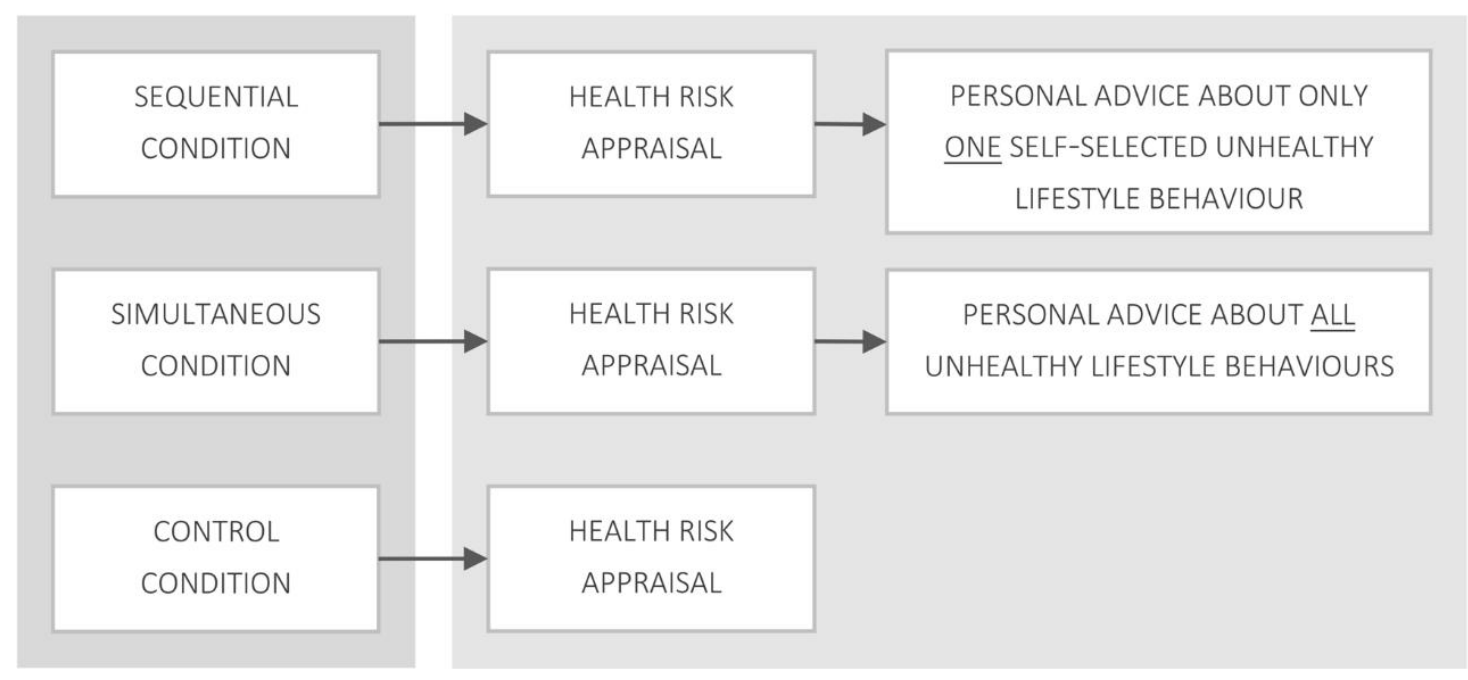

Figure 2.1 Design at baseline

\section{Sequential behaviour tailoring condition}

After receiving the health risk appraisal, individuals in the SeqC are invited to choose one of the health behaviours for which they have received a red or orange traffic light. Respondents are encouraged to change the behaviour for which they are motivated most. After receiving additional questions regarding the various psychosocial constructs of the selected lifestyle behaviour, they receive personal advice on this behaviour which aims at helping to change this health behaviour during the first year of the study.

At the follow-up measurements, individuals in this condition are asked to fill out a questionnaire about all behaviours. Again, they receive the health risk appraisal, including an overview consisting of five traffic lights. In cases where a respondent has successfully changed the selected behaviour, the option to choose a second behaviour in the second year is provided. They are free to select one of the behaviours for which they receive a red or orange traffic light. In cases where respondents have not changed the first behaviour, they will be invited to change the behaviour for which they are motivated most in the second year. This may be the same behaviour as chosen in the first year, but the respondents may also select another lifestyle behaviour for which they have obtained a red or orange traffic light. Afterwards, they receive the questionnaire containing items about perceptions (beliefs) regarding the behaviour they have selected to focus on as well as personal advice.

If respondents fill in the test in the meantime, which means that they re-visit the programme without having received an invitation for a follow-up measurement, they only have to answer the questions concerning the selected behaviour in order to receive ipsative feedback on their chosen topic and personal advice if they are interested. 


\section{Simultaneous behaviour tailoring condition}

Upon receiving the health risk appraisal, participants of the SimC are not explicitly asked to select one behaviour which is in contrast to the SeqC. In cases where these participants do not adhere to the recommendation of one or more of the lifestyle behaviours, they obtain additional questions (psychosocial constructs) as well as personal advice on all behaviours for which they receive a red or orange traffic light in the health risk appraisal.

At every follow-up visit/re-visit, individuals are asked to fill out questions about all five lifestyle behaviours. In this way, the programme can check on which behaviours the individual should receive personal advice concerning his or her perceptions. Moreover, they receive ipsative feedback concerning all five behaviours.

\section{Control condition}

During the first visit of the programme, respondents in the control condition receive the health risk appraisal. Afterwards, this group only receives e-mails requesting them to fill out questionnaires about the five lifestyle behaviours at follow-up at 12 months (T1) and 24 months (T2). The control group does not receive any personal advice throughout the term of this study.

\section{Primary and secondary outcomes}

Our first objective is to compare the effectiveness of the two different strategies for changing lifestyle with each other as well as with a control group. In addition to the effect studies, we will also gather and analyse data to realize additional outcomes:

\section{Process evaluation}

Process evaluations will be executed to assess levels of personalization and appreciation and to analyse which elements of the computer-tailored programme were most attractive and successful. We aim at gathering process evaluation data to analyse the factors facilitating and hindering the use of the web-based modules. All participants receive questions concerning the website (five items; e.g. "In my opinion, the website is user-friendly"; totally agree - totally disagree), the health risk appraisal (12 items; e.g. "The health risk appraisal gives a good overview of my lifestyle"; totally agree - totally disagree) and two general questions (i.e. "Do you intend to revisit the website in the future?"; yes, definitely - no, definitely not; "Do you have any general comments concerning the programme?"; open question). The two experimental groups additionally receive questions regarding the personal advice (15 items; e.g. "The pieces of advice are informative"; totally agree - totally disagree). We will compare the evaluations of the three different groups to analyse differences with regard to the process evaluation data. 


\section{Subgroup differences}

We aim at exploring potential differences between high SES and low SES groups, different age groups, men and women, and healthy and unhealthy people (defined by the items about health status).

\section{Power analysis}

The power analysis is based on detecting 10\% differences in behaviour between the different conditions. Power analysis calculations differ per behaviour. Estimations for finding effects on the lifestyle behaviours of smoking and alcohol consumption were the most conservative power analyses resulting in the greatest number of respondents needed. Since approximately $25 \%$ to $30 \%$ of the population smoke and almost similar percentages are estimated for non-compliance with the alcohol guideline (Statistics Netherlands, 2007a), sample size calculations are highly determined by these two groups. In order to be able to find a significant difference of $10 \%$ in quit rates and improved behaviour between the conditions, power calculations (alpha $=.80 ; p=.05$ ) indicate that 219 smoking persons per cell are needed, resulting in a total of 657 smokers. Hence, if approximately $25 \%$ of the adult population smokes this requires a total study sample of 2,826 persons. A similar conclusion can be drawn for the persons not adhering to the Dutch alcohol recommendations. Correcting for dropout, this indicates that the total sample size for this study will be approximately 3,285 persons. The sample will be sufficient to assess changes in the other behaviours (fruit and vegetable consumption and physical activity).

\section{Attrition prevention}

Different strategies are used in order to prevent attrition and to encourage participation in the long run. First, participants are eligible to win prizes. Participants completing the study stand a chance to win one of 300 prizes of 50 Euros. Second, half of the respondents of the experimental groups receive e-mail prompts, including invitations to visit the tailored programme, every three months, in order to be able to analyse whether pro-active prompting will enhance visiting the website and using computer-tailored feedback more intensively. Third, new information is placed on the website monthly in order to keep it up to date and make it attractive for people to revisit the website.

\section{Statistical analyses}

General descriptive statistics will be used to describe the characteristics of the participants and the main findings concerning the public health guidelines. With regard to the assessment of behavioural effects and differences between the three groups, logistic as well as linear regression analyses will be performed using Statistical Package for the Social Sciences (SPSS) version 15. With regard to the process evaluation, descriptive statistics will be used, such as frequencies, means, standard deviations 
and ranges. Satisfaction with the programme and ideas for improvement will also be analysed by summarizing the answers to the open-ended question and comparing different attitudes and suggestions. In order to explore potential differences between groups (e.g. low SES versus high SES, age groups, gender), regression techniques and latent cluster analyses will be used.

\section{DISCUSSION}

The results of this study are of importance for the future development and implementation of computer-tailored programmes, which aim at stimulating healthy lifestyles in order to prevent chronic diseases. Positively changing a set of health risk behaviours, i.e. smoking, high alcohol consumption, bad nutrition and physical inactivity, is becoming increasingly common since these changes are relevant for the primary prevention of cardiovascular related diseases and cancer (National Institute for Public Health and the Environment, 2002; World Health Organization, 2007). Due to the target of several behaviours and health problems, by using a tailored web-based programme, these kinds of programmes are considered to be potentially cost-effective (Cobiac et al., 2009).

Internet-based health education programmes have become popular in recent years. To our knowledge, however, few were tested in an RCT to analyse effects on multiple behaviours. Addressing smoking, alcohol consumption, fruit and vegetable intake, and physical activity at the same time, is one fundamental part of recent developments. In addition, we compare a simultaneous behaviour tailoring approach with a sequential behaviour tailoring approach. Hence, we will experimentally test which tailoring lifestyle approach is more effective and appreciated. This study will thus yield results about the need for differential lifestyle approaches. An optimal use of (one of) the different strategies could increase the effectiveness of web-based computer-tailored lifestyle programmes. Research is also important in order to assess whether the effectiveness and appreciation of the programme differs between various subgroups.

Furthermore, a unique element of this project is that our computer-tailored programme is offered as an additional service of the RHAs in the Dutch regions of North-Brabant and Zeeland within the regular health monitoring they provide to adults. The integration of the computer-tailored programme to the Adult Health Monitor 2009 brings along various advantages. First, the RHAs may extend and improve their health education task by offering tailored feedback regarding lifestyle behaviours to their respondents. Second, the personal advice may be an extra stimulus for people to participate in the Adult Health Monitor. Third, the Adult Health Monitor of the two provinces has a total reach of nearly 100,000 inhabitants. Due to the integration of our computer-tailored programme into the Adult Health Monitor, a large number of people within the Dutch population may be reached. 


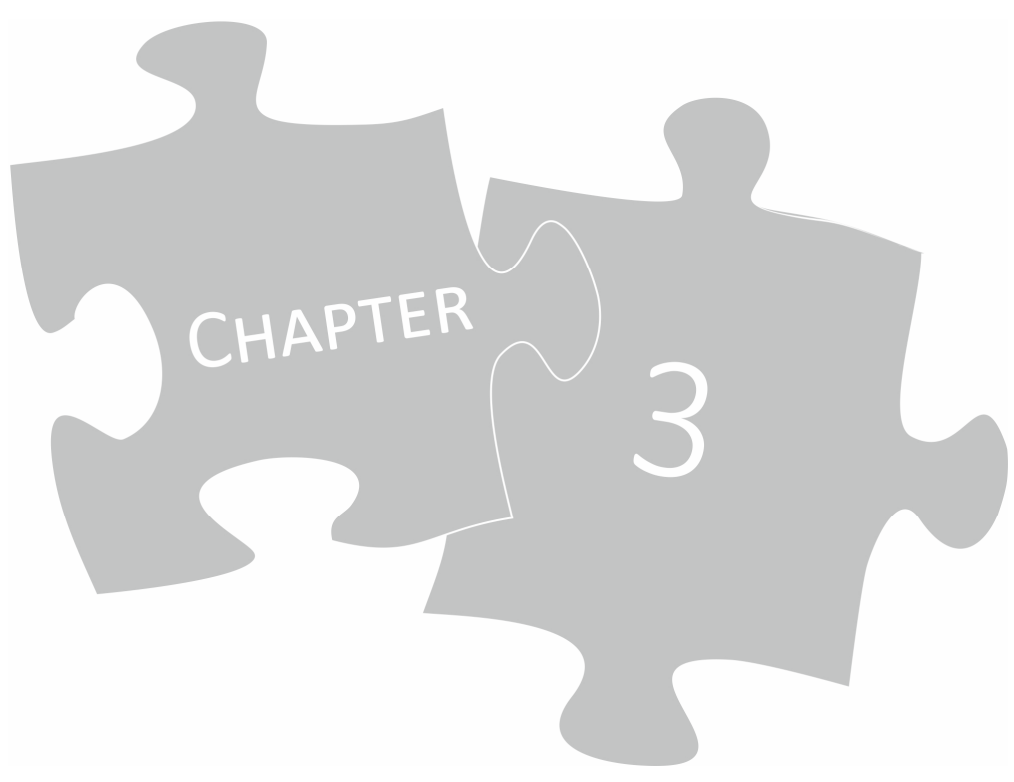

THE USE OF A PROACTIVE DISSEMINATION STRATEGY TO OPTIMIZE REACH OF AN INTERNET-DELIVERED COMPUTER-TAILORED LIFESTYLE INTERVENTION

This chapter has been published as:

Schneider F, Schulz DN, Pouwels LHL, de Vries H, van Osch LA. The use of a proactive dissemination strategy to optimize reach of an internet-delivered computer tailored lifestyle intervention. BMC Public Health 2013; 13:721. 


\section{ABSTRACT}

Background: The use of reactive strategies to disseminate effective Internet-delivered lifestyle interventions restricts their level of reach within the target population. This stresses the need to invest in proactive strategies to offer these interventions to the target population. The present study used a proactive strategy to increase reach of an Internet-delivered multi component computer-tailored intervention, by embedding the intervention in an existing online health monitoring system of the Regional Health Authorities in the Netherlands.

Methods: The research population consisted of Dutch adults who were invited to participate in the Adult Health Monitor ( $N=96,388$ ) offered by the Regional Health Authorities. This Monitor consisted of an online or a written questionnaire. A prospective design was used to determine levels of reach, by focusing on actual participation in the lifestyle intervention. Furthermore, adequacy of reach among the target group was assessed by composing detailed profiles of intervention users. Participants' characteristics, like demographics, behavioural and mental health status and quality of life, were included in the model as predictors.

Results: A total of 41,155 (43\%) people participated in the Adult Health Monitor, of which $41 \%$ ( $n=$ $16,940)$ filled out the online version. More than half of the online participants indicated their interest ( $n=9,169 ; 54 \%)$ in the computer-tailored intervention and 5,168 participants (31\%) actually participated in the Internet-delivered computer-tailored intervention. Males, older respondents and individuals with a higher educational degree were significantly more likely to participate in the intervention. Furthermore, results indicated that especially participants with a relatively healthier lifestyle and a healthy body mass index were likely to participate.

Conclusions: With one out of three online Adult Health Monitor participants actually participating in the computer-tailored lifestyle intervention, the employed proactive dissemination strategy succeeded in ensuring relatively high levels of reach. Reach among at-risk individuals (e.g. low socioeconomic status and unhealthy lifestyle) was modest. It is therefore essential to further optimize reach by putting additional effort into increasing interest in the lifestyle intervention among at-risk individuals and to encourage them to actually use the intervention.

Keywords: Internet-delivered interventions; online interventions; reach; computer-tailoring; lifestyle; user characteristics; proactive dissemination 


\section{BACKGROUND}

Lifestyle behaviours, such as smoking, unhealthy eating habits, physical inactivity and excessive alcohol consumption are established risk factors for chronic diseases, most importantly for cardiovascular diseases, different types of cancer and diabetes type II (National Institute for Public Health and the Environment, 2012; World Health Organization, 2011). The high prevalence of these behaviours (National Institute for Public Health and the Environment, 2012) and their tendency to co-occur (De Vries et al., 2008c; Strecher et al., 2002) stress the need to invest in effective lifestyle interventions.

Currently, lifestyle interventions are more and more frequently disseminated through the Internet. Due to the high level of reach and accessibility of the Internet (Internet World Stats, 2012), it offers a good platform for the dissemination of tailored and targeted interventions to the general public (Brug et al., 2005; Lustria et al., 2009; Ritterband et al., 2003). Especially interventions using computertailored techniques have reported positive effects (Krebs et al., 2010; Lustria et al., 2009; Ritterband et al., 2003), e.g. in the fields of physical activity (Vandelanotte et al., 2007b; Van den Berg et al., 2007), fruit and vegetable intake (Brug et al., 1998; Neville et al., 2009a), smoking (Borland et al., 2004; Dijkstra et al., 1998a) and alcohol consumption (Riper et al., 2008; White et al., 2010). This potentially high level of reach, combined with the proven effectiveness of computer-tailored interventions, would suggest that interventions offered through the Internet hold the promise to significantly contribute to an increased impact on public health (Glasgow et al., 1999).

However, despite the promising prospects of the Internet, actual reach of effective interventions within the target population remains inadequate (Evers et al., 2005; Eysenbach, 2005; Glasgow et al., 2007; Portnoy et al., 2008), as only a limited proportion of people is actually reached by the interventions (Brouwer et al., 2009; Evers et al., 2005). These suboptimal levels of reach might be partially explained by the fact that most effective interventions are offered reactively to the target population (Prochaska et al., 2001). The use of reactive dissemination strategies implies that a rather passive approach is taken in which users themselves must undertake action in order to use and optimally benefit from the intervention content (Prochaska et al., 1997). Potential users must actively look and register for existing lifestyle initiatives. However, several barriers prevent large proportions of the population from actively seeking assistance in changing their lifestyle behaviours. The barriers pertain to insufficient knowledge on the existence of interventions or places where they can be found, unawareness of the current status of their lifestyle behaviours or a lack of motivation to change their lifestyle (Ajzen, 1991; De Vries et al., 2003). This stresses the necessity to invest in strategies through which lifestyle interventions can be proactively offered to the target population. These strategies have the potential to diminish important barriers for use and increase the knowledge of existing, evidencebased interventions (Schneider et al., 2012; Velicer et al., 1999b).

Within the present study, a proactive strategy was used to increase reach of an Internet-delivered multi component computer-tailored intervention, focusing on physical activity, fruit and vegetable consumption, alcohol intake and smoking cessation. In order to proactively offer the intervention to the target population, the intervention was embedded in a nationwide survey, the Adult Health 
Monitor, of two of the Regional Health Authorities (RHAs) in the Netherlands (National Institute for Public Health and the Environment, 2005). As part of their statutory obligation to periodically monitor the health of the Dutch adult population, this survey is used to assess overall levels of health, as well as different aspects of general health, e.g. physical, mental and social health among a representative sample with an interval of four years. Based on findings of this monitoring tool, future health policies at a national, provincial and local level are outlined. Integrating the Internet-delivered computertailored intervention in the Adult Health Monitor was expected to have several benefits. Most importantly, the Adult Health Monitor was expected to provide an important access point for reaching a considerably large segment of the Dutch population with an evidence-based intervention. Furthermore, by integrating the computer-tailored intervention in the Adult Health Monitor, awareness of the existence of the intervention could be increased. Thirdly, by embedding the intervention in the Adult Health Monitor, data can directly be obtained and presented in order to inform people on the status of their current lifestyle. This may increase the level of lifestyle awareness and serve as an important cue to action to positively change their current lifestyle. Finally, integrating an Internet-delivered computer-tailored intervention will enable the RHAs to optimize their public health education task; besides solely monitoring health behaviour, the RHAs now have an opportunity to provide people with personalized advice on how to effectively change their lifestyle status.

The main aim of this study was to determine the effect of this proactive dissemination strategy on reach of the Internet-delivered computer-tailored intervention. Within the RE-AIM framework, reach is defined as the percentage of people invited and eligible for participation that actually participate in the intervention (Glasgow et al., 1999). Therefore, success of this proactive dissemination strategy was assessed by studying actual levels of participation in the computer-tailored lifestyle intervention among people included in the Adult Health Monitor sample. Furthermore, the second aim of this study was to determine adequacy of reach among the target population (Velicer et al., 1999b) by composing and analysing detailed profiles of intervention users (Glasgow et al., 1999; Rogers, 1995).

\section{METHODS}

Within the current study, the level of reach of an Internet-delivered computer-tailored lifestyle intervention offered through the Adult Health Monitor was examined. Furthermore, adequacy of reach among the target group was assessed by composing detailed profiles of computer-tailored intervention users. This study was approved by the Medical Ethics Committee of Maastricht University and the University Hospital Maastricht (NL27235.068.09/MEC 09-3-016) (Schneider et al., 2011; Schulz et al., 2011). Data for the present study were collected from October 2009 until July 2010.

\section{Participants and inclusion criteria}

The research population consisted of adults (19-64 years) living in the Dutch Provinces of Zeeland and North-Brabant who participated in the Adult Health Monitor 2009. Additional inclusion criteria for 
participation in the computer-tailored intervention were that participants had to be able to understand the Dutch language sufficiently, had to have access to the Internet and had to have a valid e-mail address. There were no explicit exclusion criteria stated for the current study. Participants had to consent to their participation by filling out an online informed consent form.

\section{Recruitment and procedure}

In the fall of 2009, four RHAs of the provinces North-Brabant and Zeeland invited a representative sample of inhabitants of these provinces to participate in the Monitor 2009. A total of 96,388 participants received an invitation to participate by postal mail. This invitation included information on the content and purpose of the Adult Health Monitor. Furthermore, a return envelope was included to return the questionnaire after completion. Besides filling out the written Adult Health Monitor questionnaire, participants were also offered an opportunity to fill out an online version of the questionnaire. For this purpose, a link to the website was included in the invitation, as well as personal login information. Since the Internet-delivered computer-tailored lifestyle intervention was a webbased intervention, it was only available for participants that decided to fill out the online version of the monitor questionnaire. Online participation was therefore encouraged by informing people about the embedded intervention in the invitation letter and by pointing out its exclusive availability for online participants.

After completing the Adult Health Monitor, all participants of the online version were introduced to the embedded computer-tailored lifestyle intervention. Participants were explained that the intervention was free of charge and provided an opportunity to receive personalized computertailored feedback about their health behaviours. Participants indicating to be interested in the intervention were asked to leave their e-mail address and to fill out an online form indicating their consent to participate in the current study. Approximately three weeks after completion of the Adult Health Monitor, all interested participants received an e-mail invitation to log in to the computertailored intervention with a personal login code and password. After approximately one month, nonresponders received an additional e-mail reminding them about their interest in the intervention and inviting them to participate. After logging on to the intervention, participants received detailed information on its goal and content. Data on demographic and behavioural characteristics obtained through the Adult Health Monitor were transported to the computer-tailored intervention.

\section{Materials}

The computer-tailored intervention integrated interventions that have been tested and proven to be effective in randomized control trials for increasing smoking cessation, promoting the intake of fruit and vegetables, increasing the level of physical activity and reducing the level of alcohol consumption (Brug et al., 1999b; Poel et al., 2009; Riper et al., 2008; Smeets et al., 2008; Van Keulen et al., 2011). The intervention used a dual approach to guide people towards behaviour change. The first part 
consisted of a health risk appraisal and compared participants' health behaviour status to the Dutch public health guidelines set for these behaviours (i.e. being moderately physically active for 30 minutes at least five days a week (Hildebrandt et al., 2010), eating two pieces of fruit per day and eating 200 grams of vegetables per day (The Health Council of the Netherlands, 2006), not drinking more than one (women) or two (men) glasses of alcohol a day (Netherlands Institute of Mental Health and Addiction, 2013a) and non-smoking (The Dutch Institute for Healthcare Improvement, 2004)). We used feedback messages to inform people on the current status of each health behaviour. All messages were complemented by using graphic representations of traffic lights (Strecher et al., 2008), with a green light corresponding to adherence to the guidelines and a red light corresponding to nonadherence. An orange light was used for people that were close to adherence to these guidelines. In case of discrepancies between current behaviour and the guidelines, people were alerted and directed to the computer-tailored modules (Schulz et al., 2011).

Second, participants were provided with assistance in changing their health behaviour by means of five separate computer-tailored modules on physical activity, fruit and vegetable intake, alcohol consumption and smoking cessation. The content of these modules was based on the integrated model for exploring motivational and behavioural change (I-Change model) (De Vries et al., 2003) and used a fixed, gradual approach to guide people towards behaviour change. Within the modules, all health advices were adapted to individuals' demographic, behavioural and cognitive characteristics (De Vries \& Brug, 1999; Kreuter et al., 1999b). Demographic and behavioural characteristics, like participants' gender and current health behaviour status were directly obtained through the Adult Health Monitor. Cognitive variables, such as attitude, perceived social influence, self-efficacy, intention and action planning strategies (preparatory plans and coping plans) were assessed by using an additional tailoring questionnaire. An extensive description of the intervention content is described elsewhere (Schneider et al., 2011; Schulz et al., 2011).

\section{Measures}

User characteristics were collected in order to produce detailed profiles of people who decided to participate in the computer-tailored intervention in order to determine adequacy of reach. These profiles entailed information on demographic characteristics, health behaviour status, mental health status and quality of life, obtained through the Adult Health Monitor.

\section{Demographic characteristics}

Demographic characteristics included questions on age, gender ( 1 = male; 2 = female), height, weight, marital status (widower, divorced, unmarried (not in a relationship $=1$ ); or living together, married (in a relationship $=2)$, native country $(1=$ not from the Netherlands; $2=$ from the Netherlands), educational level (no education, primary or lower vocational school (low $=1$ ); secondary vocational school or high school (medium $=2$ ); or higher professional education or university (high $=3$ )) and 
current work status (unemployed $=1$; employed $=2$ ). Data on participants' height (in meters) and weight (in kilograms) were used to compose a body mass index (BMI). This index was defined by dividing participants' body weight $(\mathrm{kg})$ by the square of participants' height $(\mathrm{m})$ and was categorized as $<18.5=1$ (underweight); $\geq 18.5$ to $<25=2$ (normal weight) $; \geq 25$ to $<30=3$ (overweight) $\geq 30=4$ (obesity).

\section{Health behaviour status}

Health behaviour status was objectively assessed by measuring level of physical activity, fruit and vegetable intake, alcohol consumption and smoking behaviour. Physical activity was measured by the Short QUestionnaire to ASsess Health-enhancing physical activity (SQUASH) (Wendel-Vos et al., 2003). Fruit and vegetable intake were assessed using the Food-Frequency-Questionnaire (FFQ) for fruit and vegetable intake (Bogers et al., 2004; Van Assema et al., 2002). Alcohol consumption was measured by the Dutch Quantity-Frequency-Variability (QFV) Questionnaire (Lemmens et al., 1992) and smoking status was assessed by asking participants whether they smoked, what they smoked (cigarettes, cigars, packets pipe tobacco) and how much they smoked per day (cigarettes) and per week (cigars/ packets pipe tobacco). Furthermore, an additional item was used to obtain a more subjective assessment of participants' lifestyle status. This item assessed the degree to which participants considered their own current lifestyle to be healthy and was measured on a five point scale ( 1 = very unhealthy; 5 = very healthy).

\section{Mental health status}

Mental health status was assessed by using the Kessler Psychological Distress Scale (K10) (Andrews \& Slade, 2001; Kessler et al., 2002). This scale assesses the occurrence of non-specific psychological distress using ten items measured on a five point scale ( $1=$ none of the time; $5=$ all of the time). $\mathrm{A}$ sum score was computed, with higher scores indicating higher levels of distress.

\section{Quality of life}

Quality of life was assessed using the Medical Outcomes Study 12-Item Short Form Health Survey (SF12) (Jenkinson et al., 1997; Ware et al., 1996).

\section{Outcome measures}

The primary outcome measure was level of actual participation in the intervention programme. Secondary outcome measures were medium of participation and level of interest in the intervention. Dichotomous variables were created in order to examine level of participation $(0=$ no participation; 
$1=$ participation $)$, medium of participation ( 0 = written questionnaire; $1=$ online questionnaire) and level of interest in the intervention ( $0=$ not interested; $1=$ interested $)$. Participants of the online Adult Health Monitor were asked to indicate their interest in the intervention by leaving their e-mail address. Interest in the intervention was labelled as "yes" when people filled out their e-mail address. Furthermore, participation was objectively monitored by looking at login data and was labelled as "yes" when people responded to the e-mail invitation by logging in to the computer-tailored intervention.

\section{Statistical analysis}

First, general descriptive statistics were used to describe user characteristics of the participants, by focusing on demographic characteristics, health behaviour status, mental health status and quality of life as well as level of interest and participation in the computer-tailored intervention. Second, logistic regression analyses were conducted. Medium of participation, interest and participation in the computer-tailored intervention were the dependent variables. Demographic characteristics (age, gender, BMI, marital status, native country, educational level and current work status), health behaviour status, mental health status and quality of life were included in the model as predictors. An alpha of .05 was used to indicate statistical significance. All statistical analyses were done with the programme SPSS 19.0.

\section{RESULTS}

\section{Reach of the proactive dissemination strategy}

In total, 41,155 (43\%) people participated in the Adult Health Monitor 2009 in the provinces of NorthBrabant and Zeeland; 24,215 participants (59\%) filled out the written version and 16,940 participants (41\%) filled out the online version. All online participants were introduced to the Internet-delivered computer-tailored lifestyle intervention and 9,169 participants (54\%) actually indicated to be interested in the intervention. Finally, all participants that indicated their interest were invited to use the intervention. This resulted in a total of 5,168 participants (31\% of the online sample and $56 \%$ of the sample of people interested in participating) logging in to the Internet-delivered computertailored intervention (Figure 3.1). 


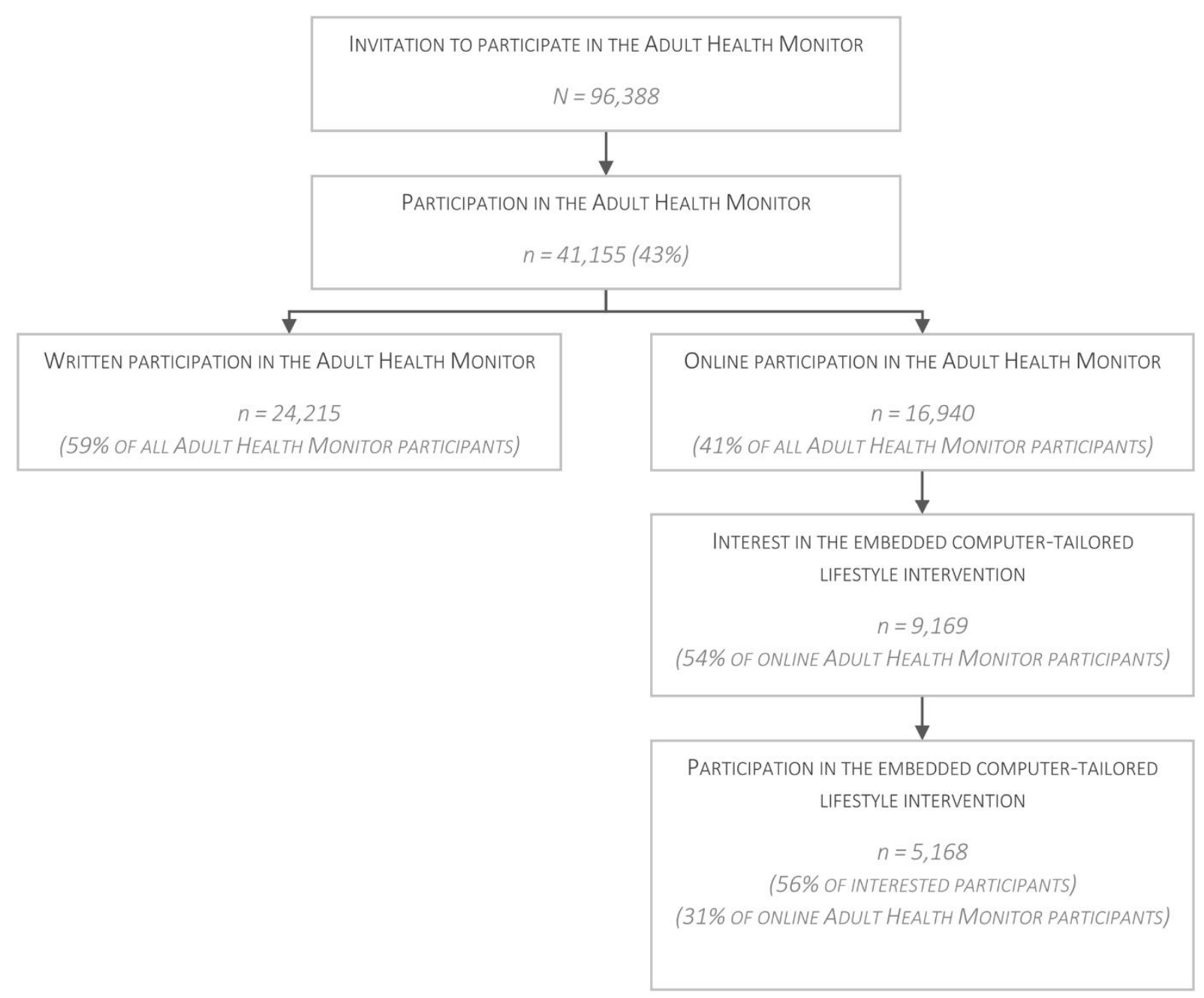

Figure 3.1 Flowchart of Adult Health Monitor and computer-tailored lifestyle intervention participation

\section{Baseline characteristics of intervention users}

Users of the Internet-delivered computer-tailored intervention had a mean age of 44 years (SD = 12.67), around 54\% was male and almost 50\% had a medium educational level (Table 3.1). Furthermore, a little over 75\% of all participants was employed and in a relationship and 95\% was born in the Netherlands. Approximately $50 \%$ of all participants had a healthy body weight, whereas a little less than $2 \%$ of all visitors was underweight, $35 \%$ was overweight and $10 \%$ was obese. With regard to physical activity, $21 \%$ did not comply with the Dutch guidelines, whereas $46 \%$ and $69 \%$ were not adhering to the Dutch guidelines of fruit and vegetables intake, respectively. One out of five visitors (19\%) indicated that they smoked, whereas approximately one of four participants (28\%) did not comply with the Dutch guidelines for alcohol intake. In total, the largest group of people complied with three lifestyle guidelines (36\%), whereas a very limited percentage of people complied with none of these guidelines (1\%) or all of the guidelines (10\%). Characteristics of all Adult Health Monitor participants, online Adult Health Monitor participants and people that indicated their interest in the Internet-delivered computer-tailored lifestyle intervention are also listed in Table 3.1. 
Table 3.1 Characteristics of Adult Health Monitor participants $(N=41,155)$

\begin{tabular}{|c|c|c|c|c|}
\hline & $\begin{array}{r}\text { Total sample } \\
\mathrm{N}=41,155 \\
\end{array}$ & $\begin{array}{r}\text { Online completion } \\
n=16,940\end{array}$ & $\begin{array}{r}\text { Interest } \\
\mathrm{n}=9,169\end{array}$ & $\begin{array}{r}\text { Participation } \\
n=5,168 \\
\end{array}$ \\
\hline Age (19-64) & $44.78(S D=12.70)$ & $43.19(\mathrm{SD}=12.42)$ & $43.23(\mathrm{SD}=12.77)$ & $43.94(S D=12.67)$ \\
\hline \multicolumn{5}{|l|}{ Sex } \\
\hline Men & $44.7 \%$ & $51.1 \%$ & $52.6 \%$ & $53.8 \%$ \\
\hline Women & $55.3 \%$ & $48.9 \%$ & $47.4 \%$ & $46.2 \%$ \\
\hline \multicolumn{5}{|l|}{ Education level } \\
\hline Low & $23.2 \%$ & $14.7 \%$ & $13.2 \%$ & $10.6 \%$ \\
\hline Medium & $48.5 \%$ & $48.9 \%$ & $47.7 \%$ & $46.9 \%$ \\
\hline High & $28.2 \%$ & $36.4 \%$ & $39.1 \%$ & $42.6 \%$ \\
\hline \multicolumn{5}{|l|}{ Work situation } \\
\hline Employed & $72.8 \%$ & $77.1 \%$ & $76.5 \%$ & $76.3 \%$ \\
\hline Unemployed & $27.2 \%$ & $22.9 \%$ & $23.5 \%$ & $23.7 \%$ \\
\hline \multicolumn{5}{|l|}{ Marital status } \\
\hline In a relationship & $76.8 \%$ & $77.0 \%$ & $75.4 \%$ & $76.0 \%$ \\
\hline Not in a relationship & $24.2 \%$ & $23.0 \%$ & $24.6 \%$ & $24.0 \%$ \\
\hline \multicolumn{5}{|l|}{ Native country } \\
\hline The Netherlands & $93.3 \%$ & $93.5 \%$ & $93.8 \%$ & $95.0 \%$ \\
\hline Other & $6.7 \%$ & $6.5 \%$ & $6.2 \%$ & $5.0 \%$ \\
\hline \multicolumn{5}{|l|}{$\mathrm{BMI}\left(\mathrm{kg} / \mathrm{m}^{2}\right)$} \\
\hline$<18.5$ & $1.5 \%$ & $1.4 \%$ & $1.5 \%$ & $1.7 \%$ \\
\hline$\geq 18.5$ to 25 & $50.9 \%$ & $51.5 \%$ & $51.1 \%$ & $52.6 \%$ \\
\hline$\geq 25$ to $<30$ & $35.1 \%$ & $35.4 \%$ & $35.6 \%$ & $35.4 \%$ \\
\hline$\geq 30$ & $12.4 \%$ & $11.7 \%$ & $11.8 \%$ & $10.4 \%$ \\
\hline \multicolumn{5}{|l|}{ Physical activity } \\
\hline Adherence & $78.4 \%$ & $77.1 \%$ & $77.7 \%$ & $79.1 \%$ \\
\hline Non-adherence & $21.6 \%$ & $22.9 \%$ & $22.3 \%$ & $20.9 \%$ \\
\hline \multicolumn{5}{|l|}{ Vegetable consumption } \\
\hline Adherence & $31.2 \%$ & $29.9 \%$ & $30.4 \%$ & $31.5 \%$ \\
\hline Non-adherence & $68.8 \%$ & $70.1 \%$ & $69.6 \%$ & $68.5 \%$ \\
\hline \multicolumn{5}{|l|}{ Fruit consumption } \\
\hline Adherence & $49.7 \%$ & $46.1 \%$ & $45.8 \%$ & $45.9 \%$ \\
\hline Non-adherence & $50.3 \%$ & $53.9 \%$ & $54.2 \%$ & $54.1 \%$ \\
\hline \multicolumn{5}{|l|}{ Smoking behaviour } \\
\hline Adherence & $74.7 \%$ & $77.6 \%$ & $78.1 \%$ & $81.7 \%$ \\
\hline Non-adherence & $25.3 \%$ & $22.4 \%$ & $21.9 \%$ & $18.3 \%$ \\
\hline \multicolumn{5}{|l|}{ Alcohol intake } \\
\hline Adherence & $73.6 \%$ & $73.6 \%$ & $72.3 \%$ & $71.7 \%$ \\
\hline Non-adherence & $26.4 \%$ & $26.4 \%$ & $27.7 \%$ & $28.3 \%$ \\
\hline \multicolumn{5}{|l|}{ Total number of guidelines } \\
\hline 0 & $0.9 \%$ & $0.9 \%$ & $0.9 \%$ & $0.7 \%$ \\
\hline 1 & $7.2 \%$ & $7.5 \%$ & $7.3 \%$ & $6.5 \%$ \\
\hline 2 & $21.3 \%$ & $22.1 \%$ & $22.4 \%$ & $20.9 \%$ \\
\hline 3 & $33.6 \%$ & $34.1 \%$ & $33.9 \%$ & $35.5 \%$ \\
\hline 4 & $27.1 \%$ & $26.2 \%$ & $26.3 \%$ & $26.6 \%$ \\
\hline 5 & $9.9 \%$ & $9.1 \%$ & $9.2 \%$ & $9.7 \%$ \\
\hline \multicolumn{5}{|l|}{ Personal judgment lifestyle } \\
\hline Very unhealthy & $5.7 \%$ & $5.5 \%$ & $5.6 \%$ & $6.5 \%$ \\
\hline Unhealthy & $61.8 \%$ & $61.6 \%$ & $60.3 \%$ & $61.8 \%$ \\
\hline Not unhealthy / not healthy & $28.9 \%$ & $28.9 \%$ & $29.4 \%$ & $27.5 \%$ \\
\hline Healthy & $3.2 \%$ & $3.7 \%$ & $4.3 \%$ & $3.9 \%$ \\
\hline Very healthy & $0.4 \%$ & $0.3 \%$ & $0.4 \%$ & $0.3 \%$ \\
\hline K10 & $15.41(S D=6.26)$ & $15.17(S D=5.90)$ & $15.47(S D=6.08)$ & $15.21(S D=5.71)$ \\
\hline SF-12 & $39.76(S D=5.63)$ & $40.09(S D=5.29)$ & $39.88(\mathrm{SD}=5.39)$ & $40.12(S D=5.19)$ \\
\hline
\end{tabular}




\section{Adequacy of reach}

\section{Participation in the online Adult Health Monitor}

Results of multiple logistic regression analyses indicated that younger (OR 0.99; $\mathrm{Cl} 0.99-0.99$; $\mathrm{p}<.05$ ), male (OR 0.65; $\mathrm{Cl} 0.62-0.68 ; \mathrm{p}<.05)$ and relatively highly educated participants (OR 2.63; $\mathrm{Cl} 2.45-2.81 ; \mathrm{p}<.05$ ) as well as participants that were employed (OR 1.06; $\mathrm{Cl} 1.00-1.12 ; \mathrm{p}<.05$ ) and in a relationship (OR 1.09; $\mathrm{Cl} 1.03-1.15 ; \mathrm{p}<.05)$ were more inclined to fill out the online version of the Adult Health Monitor. Furthermore, participants with an unhealthier lifestyle (fewer guidelines complied with) were more likely to fill out the online version of the Adult Health Monitor (OR 0.97; $\mathrm{Cl} 0.95-0.99 ; \mathrm{p}<.05)$.

\section{Interest in the computer-tailored lifestyle intervention}

Results of multiple logistic regression analyses indicated that older (OR 1.00; $\mathrm{Cl} 1.00-1.01 ; \mathrm{p}<.05$ ) and male participants (OR 0.88; $\mathrm{Cl} 0.82-0.94 ; \mathrm{p}<.001$ ), as well as participants with a relatively higher educational degree were more likely to indicate their interest in the programme (OR 1.57; $\mathrm{Cl} 1.42$ $1.74 ; p<.001)$. Furthermore, participants indicating their interest in the programme were less likely to be in a relationship (OR 0.84; $\mathrm{Cl} 0.77-0.91 ; \mathrm{p}<.001$ ) and employed (OR 0.92; $\mathrm{Cl} 0.85-0.99 ; \mathrm{p}<.05$ ) and were more often born in the Netherlands (OR 1.19; Cl 1.05-1.36; $p<.01$ ). Finally, participants with a higher level of distress, as indicated by their K10-score, were more likely to be interested in the computer-tailored programme (OR 1.02; $\mathrm{Cl} 1.01-1.03 ; \mathrm{p}<.001)$.

\section{Use of the computer-tailored lifestyle intervention}

Results of multiple logistic regression analyses indicated that male (OR $0.91 ; \mathrm{Cl} 0.83-0.99 ; \mathrm{p}<.05$ ), older (OR 1.02; Cl $1.01-1.02 ; \mathrm{p}<.05)$ and relatively highly educated participants (OR 1.93; Cl 1.67 $2.23 ; p<.05$ ) were more likely to participate in the intervention by logging in to the intervention (see Table 3.2). Furthermore, intervention participants were more likely to be from the Netherlands (OR $1.45 ; \mathrm{Cl} 1.21-1.75 ; \mathrm{p}<.05)$. Finally, participants with a relatively healthier lifestyle (more guidelines complied with) (OR 1.06; Cl 1.01 - 1.10; $\mathrm{p}$.05) and underweighted participants (OR 1.55; Cl 1.06 2.27; $\mathrm{p}<0.05)$ were more likely to participate in the computer-tailored intervention, whereas overweighed (OR 0.88; $\mathrm{Cl} 0.79-0.97 ; \mathrm{p}<.05$ ) and obese participants (OR 0.71; $\mathrm{Cl} 0.61-0.82 ; \mathrm{p}<.05$ ) were less likely to participate. 
Table 3.2 Predictors of participation in the computer-tailored lifestyle intervention $(N=9,169)$

\begin{tabular}{|c|c|c|c|}
\hline & \multicolumn{3}{|c|}{$\begin{array}{l}\text { Computer-tailored lifestyle intervention } \\
\text { participation }\end{array}$} \\
\hline & OR & $\mathrm{p}$ & $95 \% \mathrm{Cl}$ \\
\hline \multicolumn{4}{|l|}{ DEMOGRAPHIC CHARACTERISTICS } \\
\hline Age & 1.02 & $<.001$ & $1.01-1.02$ \\
\hline \multicolumn{4}{|l|}{ Sex } \\
\hline \multicolumn{4}{|l|}{ Male (reference) } \\
\hline Female & 0.91 & .03 & $0.83-0.99$ \\
\hline \multicolumn{4}{|l|}{ Education } \\
\hline \multicolumn{4}{|l|}{ Low (reference) } \\
\hline Medium & 1.54 & $<.001$ & $1.34-1.77$ \\
\hline High & 1.93 & $<.001$ & $1.67-2.23$ \\
\hline \multicolumn{4}{|l|}{ Work situation } \\
\hline \multicolumn{4}{|l|}{ Unemployed (reference) } \\
\hline Employed & 0.90 & .05 & $0.80-1.00$ \\
\hline \multicolumn{4}{|l|}{ Marital status } \\
\hline \multicolumn{4}{|l|}{ Not in a relationship (reference) } \\
\hline In a relationship & 0.93 & .21 & $0.83-1.04$ \\
\hline \multicolumn{4}{|l|}{ Native country } \\
\hline \multicolumn{4}{|l|}{ Other native country (reference) } \\
\hline The Netherlands & 1.45 & $<.001$ & $1.21-1.75$ \\
\hline \multicolumn{4}{|l|}{ BMI } \\
\hline \multicolumn{4}{|l|}{$\geq 18.5$ to 25 (reference) } \\
\hline$<18.5$ & 1.55 & .02 & $1.06-2.27$ \\
\hline$\geq 25$ to $<30$ & 0.88 & .009 & $0.79-0.97$ \\
\hline$\geq 30$ & 0.71 & $<.001$ & $0.61-0.82$ \\
\hline \multicolumn{4}{|l|}{ HEALTH BEHAVIOUR STATUS } \\
\hline Total \# of guidelines (adherence) & 1.06 & .009 & $1.01-1.10$ \\
\hline Personal judgment own current lifestyle & 1.06 & .14 & $0.98-1.15$ \\
\hline \multicolumn{4}{|l|}{ MENTAL HEALTH STATUS } \\
\hline K10 & 1.00 & .82 & $0.99-1.01$ \\
\hline \multicolumn{4}{|l|}{ QUALITY OF LIFE } \\
\hline SF-12 & 1.00 & .56 & $0.99-1.02$ \\
\hline
\end{tabular}

\section{DISCUSSION}

Within the current study, a proactive dissemination strategy was used to optimize reach of an Internet-delivered computer-tailored lifestyle intervention. This proactive strategy consisted of embedding the intervention in the Adult Health Monitor of the RHAs of two Dutch provinces. Success of this strategy was determined by studying actual levels of participation in the computer-tailored lifestyle intervention. Furthermore, we investigated adequacy of reach by studying user characteristics that predicted interest and participation in the computer-tailored lifestyle intervention.

\section{Reach of the proactive dissemination strategy}

More than half of the online Adult Health Monitor participants indicated their interest in the intervention. Moreover, more than half of all interested participants actually participated in the computer-tailored lifestyle intervention, which is one out of three invited and eligible participants. It is difficult to compare these results to other studies that study reach in a real world setting, since actual estimates of reach are scarce (Bennett \& Glasgow, 2009). However, a multi-risk lifestyle behaviour 
intervention offered to a comparable sample reported levels of reach around 3\% of the eligible participants (Brouwer et al., 2010). Results from efficacy trials have indicated that actual reach of the interventions is suboptimal (Brouwer et al., 2010; Glasgow et al., 2007; Leslie et al., 2005), with participation rates ranging from $2.5 \%$ to $20 \%$. Considering these results, the current proactive dissemination strategy can be regarded as successful.

\section{Adequacy of reach}

Besides studying actual levels of reach, the current study also aimed at examining adequacy of reach among the target group (i.e. at-risk individuals). This was done by composing profiles of users and by examining which user characteristics predicted both interest and participation in the intervention. Results indicated that especially older, male and relatively highly educated participants were inclined to indicate their interest in the intervention. Furthermore, participants that were unemployed, not in a relationship and born in the Netherlands were more likely to be interested in the computer-tailored intervention. Finally, participants with relatively more symptoms of depression and anxiety were more likely to be interested in the computer-tailored intervention. Even though the effect of employment status, marital status and depressive symptomatology diminished with regard to actual participation in the intervention, the effect of education and native country remained, with relatively higher educated participants and participants from the Netherlands being more willing to participate in the intervention. This finding is in line with previous studies examining use of Internet-delivered lifestyle interventions, where the majority of participants had a relatively high educational level (Brouwer et al., 2010; Lorence \& Park, 2008; Spittaels et al., 2007a; Yang et al., 2008). The level of reach was modest in other subgroups, as results indicated that especially participants with a relatively healthier lifestyle and a healthy BMI were likely to actually participate in the intervention. These results are to a large extent in line with previous findings that Internet-delivered computer-tailored interventions tend to be predominantly used by people with a healthy lifestyle (Brouwer et al., 2010; Kreuter et al., 1999b; Spittaels et al., 2007a; Verheijden et al., 2007).

\section{Participation in the Adult Health Monitor}

Of the sample that was selected to participate in the Adult Health Monitor 2009, a little over forty percent accepted the invitation to participate. Even though the overall percentages of people that participated in the Monitor has decreased compared to the Adult Health Monitor conducted in 2005 (Regional Public Health Compass, 2005), the percentage of people that decided to participate using the online monitoring questionnaire has substantially increased. In 2005, only one out of four respondents preferred to use the online questionnaire (Regional Public Health Compass, 2005). This increase is in line with the rapidly expanding penetration rates of the Internet in the Netherlands. Since 2006, Internet access rates have increased from 65\% to almost 93\% of all Dutch inhabitants in 2013 (Internet World Stats, 2012), allowing for an increased amount of people filling out the online Adult Health Monitor questionnaire. 
Participation in the online version of the Adult Health Monitor was preferred by people with an unhealthier lifestyle compared to the written version. These results imply that using the online Adult Health Monitor is a good tool to reach those people that are expected to benefit most from lifestyle interventions (i.e. people that engage in health risk behaviours). However, results on actual participation in the intervention imply that the proactive strategy did not succeed in persuading these at-risk people to actually participate in the embedded computer-tailored lifestyle intervention. Even though suboptimal participation rates among people with an unhealthy lifestyle are common for most computer-tailored lifestyle interventions (Brouwer et al., 2010; Kreuter et al., 1999b; Spittaels et al., 2007a; Verheijden et al., 2007), the online environment of the Adult Health Monitor offers good opportunities to reach at-risk groups. However, these opportunities might be further optimized in the future. Direct transportation of data from the Adult Health Monitor to the computer-tailored lifestyle intervention allowed for composition of an individual health overview addressing all relevant lifestyle behaviours. However, this overview was only presented to participants that were interested in the intervention and decided to actually participate. Impact of this proactive strategy might be further improved if this overview could be presented to all Adult Health Monitor participants, instead of only interested participants. The overview provided personalized information on the status of individuals' current lifestyle behaviours, as well as additional information regarding the guidelines set for these behaviours. As a result, the detailed content of this overview was very suitable to increase awareness of people's current lifestyle status and to point out discrepancies with the guidelines set for these behaviours. This overview may then serve as an important cue to action to change their lifestyle among people with an unhealthy lifestyle.

Considering the promising results of the used proactive dissemination strategy with regard to actual levels of reach, the Adult Health Monitor can be regarded as a suitable and promising vehicle to disseminate evidence-based Internet-delivered lifestyle interventions to the general public. The proportion of people reached shows the potential for broad reach when implemented on a national level. Offering effective interventions through the Adult Health Monitor environment at a national level can therefore serve as an important access point to reach the target population. As a consequence, public health impact of these interventions can be further optimized. Even though we partly succeeded in reaching the target population of at-risk individuals, adequacy of reach may be further optimized by putting additional effort into the recruitment of online and at-risk participants. It is therefore recommendable to explore which promotion strategies should be used to attract especially older and lower educated people to the online Adult Health Monitor questionnaire. Furthermore, effort should be put into increasing interest in the computer-tailored lifestyle intervention among online Adult Health Monitor participants and to persuade them to actually use the intervention.

\section{Strengths and limitations}

One of the major strengths of this study was the opportunity to proactively offer an Internet-delivered computer-tailored lifestyle intervention to a large sample of Dutch inhabitants within the controlled 
environment of the Adult Health Monitor. This allowed us to carefully determine the number of people invited and eligible for participation, as well the number of actual intervention participants. As a result, valuable information was obtained regarding actual numbers of reach of the used dissemination strategy. Furthermore, detailed information was gathered regarding not only characteristics of people that were interested and participated in the intervention, but also of people that refrained from participation. This is exceptional, since a problem encountered in most studies evaluating dissemination strategies is the accuracy by which all non-users can be tracked. Thus, the current study provided a unique possibility to obtain information on so-called non-users (e.g. people not interested in the intervention or people that were interested, but refrained from actual participation).

The study also suffered from several limitations. First, the sample used for this study was a convenience sample, consisting of people from two Dutch provinces. However, it is important to stress that all RHAs invite an initial sample which is representative of the Dutch population, to participate in the Adult Health Monitor (National Institute for Public Health and the Environment, 2005). However, this study might have been susceptible to selection bias. Within this study, we tried to optimize reach of a computer-tailored lifestyle intervention by embedding the intervention in the Adult Health Monitor. Since this intervention was an Internet-delivered intervention, it was only offered to people that decided to fill out the electronic version of the Adult Health Monitor. Results have indicated that these specific electronic participants tend to be younger, have a relatively higher education and an unhealthier lifestyle. As a result, data on actual intervention participation were obtained from a selective sample. This sample does not provide a good cross-section of the general Dutch population (Statistics Netherlands, 2012), which implies that the obtained results should be generalized with caution. Previous studies have, however, indicated that Internet-delivered computer-tailored interventions tend to predominantly reach higher educated people (Brouwer et al., 2011; Schneider et al., 2012), which might imply that our sample partly corresponds to the subgroup of people known to be reached by Internet-delivered computer-tailored interventions. Although it does not represent the general population, the obtained results are still valuable in the context of Internet-delivered computer-tailored lifestyle interventions.

\section{Conclusions}

The present study used a proactive strategy to increase reach of an Internet-delivered multi component computer-tailored intervention, by embedding the intervention in an existing online health monitoring system of the RHAs in the Netherlands. With one out of three online Adult Health Monitor participants actually using the intervention, the employed proactive dissemination strategy succeeded in ensuring relatively high levels of reach. With regard to adequacy of reach, results from this study indicated that reach among at-risk individuals (e.g. low socioeconomic status and unhealthy lifestyle) remained modest. We therefore recommend to further optimize reach by putting additional effort into increasing interest in the lifestyle intervention among at-risk individuals and to encourage them to actually use the intervention. 



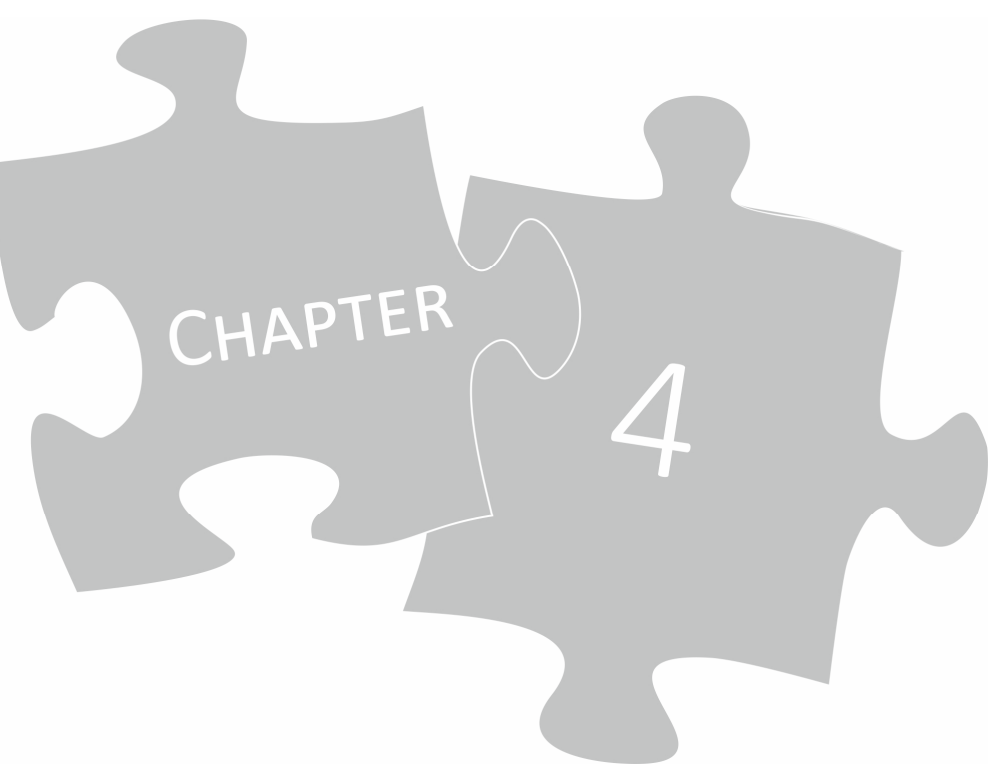

PROGRAMME COMPLETION OF A WEB-BASED TAILORED LIFESTYLE INTERVENTION FOR ADULTS: DIFFERENCES BETWEEN A SEQUENTIAL AND A SIMULTANEOUS APPROACH

This chapter has been published as:

Schulz DN, Schneider F, de Vries H, van Osch LA, van Nierop PWM, Kremers SPJ. Program completion of a web-based tailored lifestyle intervention for adults: differences between a sequential and a simultaneous approach. Journal of Medical Internet Research 2012; 14:10e26. 


\section{ABSTRACT}

Background: Unhealthy lifestyle behaviours often co-occur and are related to chronic diseases. One effective method to change multiple lifestyle behaviours is web-based computer-tailoring. Dropout from Internet interventions, however, is rather high, and it is challenging to retain participants in webbased tailored programmes, especially programmes targeting multiple behaviours. To date, it is unknown how much information people can handle in one session while taking part in a multiple behaviour change intervention, which could be presented either sequentially (one behaviour at a time) or simultaneously (all behaviours at once).

Objectives: The first objective was to compare dropout rates of two computer-tailored interventions: a sequential and a simultaneous strategy. The second objective was to assess which personal characteristics are associated with completion rates of the two interventions.

Methods: Using an RCT design, demographics, health status, physical activity, vegetable consumption, fruit consumption, alcohol intake, and smoking were self-assessed through web-based questionnaires among 3,473 adults, recruited through Regional Health Authorities in the Netherlands in the autumn of 2009. First, a health risk appraisal was offered, indicating whether respondents were meeting the five national health guidelines. Second, psychosocial determinants of the lifestyle behaviours were assessed and personal advice was provided, about one or more lifestyle behaviours.

Results: Our findings indicate a high non-completion rate for both types of intervention $171.0 \%$; $n=2,167)$, with more incompletes in the simultaneous intervention $(77.1 \% ; n=1,169)$ than in the sequential intervention (65.0\%; $n=998$ ). In both conditions, discontinuation was predicted by a lower age (sequential condition: OR 1.04; $\mathrm{p}<.001 ; \mathrm{Cl} 1.02-1.05$; simultaneous condition: OR 1.04; $\mathrm{p}<.001$; $\mathrm{Cl} 1.02-1.05$ ) and an unhealthy lifestyle (sequential condition: $\mathrm{OR} 0.86 ; \mathrm{p}=.01 ; \mathrm{Cl} 0.76-0.97$; simultaneous condition: OR 0.49; $\mathrm{p}<.001 ; \mathrm{Cl} 0.42-0.58)$. In the sequential intervention, being male (OR 1.27; $\mathrm{p}=.04 ; \mathrm{Cl} 1.01-1.59$ ) also predicted dropout. When respondents failed to adhere to at least two of the guidelines, those receiving the simultaneous intervention were more inclined to drop out than were those receiving the sequential intervention.

Conclusion: Possible reasons for the higher dropout rate in our simultaneous intervention may be the amount of time required and information overload. Strategies to optimize programme completion as well as continued use of computer-tailored interventions should be studied.

Keywords: Internet; dropout; computer-tailoring; multiple health behaviour change intervention; sequential; simultaneous; lifestyle 


\section{INTRODUCTION}

Unhealthy lifestyle behaviours, such as physical inactivity, insufficient fruit and vegetable intake, high alcohol consumption, and smoking, often co-occur (De Vries et al., 2008c; Orleans, 2004; Pronk et al., 2004b; Strecher et al., 2002) and are related to chronic diseases like cardiovascular diseases and cancer (Ezzati et al., 2002). In view of this co-occurrence, various studies have emphasized the need to develop interventions addressing more than one risk behaviour.

One method to change lifestyle behaviour is the use of computer-tailoring. Positive aspects of webbased computer-tailored programmes are that these can be administered in privacy and at a time that suits the respondent (Riper et al., 2008), and that they can be integrated in larger multicomponent interventions (De Vries \& Brug, 1999). In addition to their positive effects on health behaviour change (e.g. Brug et al., 1999a; Campbell et al., 1994; De Vries \& Brug, 1999; Krebs et al., 2010), numerous studies have shown that (web-based) tailored messages attract the public's attention (Kreuter et al., 2000b; Ruiter et al., 2006), are perceived as personally relevant (Oenema et al., 2005), and are usually better read, saved, remembered, and discussed with others than non-tailored materials (Campbell et al., 1994; De Vries \& Brug, 1999; Skinner et al., 1999; Smeets, 2006; Spittaels et al., 2007a).

The public health impact of an intervention is defined by the programme's efficacy multiplied by its reach (Abrams et al., 1996; De Vries \& Brug, 1999). Thus, the impact is highest when an intervention is effective and has a wide reach. In view of the high rate of Internet access these days $191 \%$ in the Netherlands) (Statistics Netherlands, 2011), Internet-based programmes may have the potential to reach large numbers of people. However, various studies have pointed out that the actual use of webbased interventions may be limited (De Nooijer et al., 2005; Glasgow, 2007; Leslie et al., 2005) and that leaving an Internet intervention prematurely is common (e.g. Brouwer et al., 2009; Evers et al., 2005; Glasgow et al., 2007). Brouwer et al. (2010) reported that more than half of the visitors (Dutch adults) of their online intervention left the website within 30 seconds, while 10.5\% stayed for more than 15 minutes. Hence, there is a need to identify factors associated with early discontinuation or continuation of participation in web-based programmes promoting the adoption of healthy lifestyles.

Various tailoring strategies can be used to address multiple behaviours with computer-tailored interventions, such as a sequential or a simultaneous strategy. A simultaneous strategy concurrently targets multiple behaviours for intervention, while a sequential strategy targets a single behaviour at a time. The few studies that have investigated the effects on behavioural change of sequential versus simultaneous strategies to provide multiple health behaviour change interventions reported inconsistent findings (Hyman et al., 2007; Spring et al., 2004; Vandelanotte et al., 2008). According to Vandelanotte et al. (2008), the sequential strategy may be more effective than the simultaneous strategy when participants can choose the behaviour on which they would prefer to receive personal feedback first, and can start with this part of the intervention, instead of the lifestyle modules being presented in a predefined order (see also Glasgow et al., 2004).

In any case, behavioural change will be more likely when someone completes the whole intervention programme (Strecher et al., 2008), as early dropout is a hazard to the effectiveness of any 
intervention. Hence, when considering the use of a sequential or simultaneous approach for webbased computer-tailored interventions, it is important to study continuation rates. In both types of intervention, people receive only the modules about health behaviour topics for which they are at risk in order to increase the relevance of the intervention (Prochaska et al., 2008). When being at risk for at least two behaviours, people in the simultaneous intervention receive, and thus have to handle, more information at one point in time compared to a sequential intervention, in which the same amount of information is spread over time. Therefore, a simultaneous strategy, including a more complex programme, is likely to require more time from the respondents and to increase the behaviour change demands (Prochaska et al., 2008), especially when respondents fail to meet multiple guidelines. Hence, because a simultaneous approach may lead to an overload of information, such a strategy may potentially lead to higher dropout rates than a sequential strategy (Meichenbaum \& Turk, 1987; Prochaska et al., 2008). Respondents may become overwhelmed by the amount of information (Baumeister et al., 1998) and may perceive ego depletion, leading to a reduced capacity to change (Hagger et al., 2010). Furthermore, tailoring multiple behaviours simultaneously could fail to address any single behaviour in sufficient depth (Prochaska et al., 2008; Strecher et al., 2003; Taylor et al., 2004). On the other hand, addressing various behaviours simultaneously may optimize the occurrence of synergistic effects (Dunn et al., 2006; Jakicic et al., 2002; Kremers et al., 2004; Kwak et al., 2007; Mata et al., 2009). Hence, both strategies may have advantages as well as disadvantages. To our knowledge, there is no literature about the difference in completion and dropout rates between users of sequential and those of simultaneous behaviour change interventions.

In addition to the problem of dropout, another important aspect is that high-risk populations (such as the less educated and people with many unhealthy behaviours) are often insufficiently reached (e.g. Spittaels et al., 2007b), and it is especially those with unhealthy behaviour who should engage in online health interventions and spend enough time on the website (Crutzen, 2009). It is essential to identify the characteristics of people who complete or fail to complete online health interventions. In a study by Brouwer et al. (2010), respondents who completed the programme were mostly female, middle-aged (40-50 years), and medium educated, and had a healthier lifestyle. This information about completers' characteristics can be used to improve tailored programmes by making them more attractive to the individual user.

In conclusion, computer-tailored technology addressing multiple behaviours is still in its infancy (Noar et al., 2008; Prochaska, 2008; Prochaska et al., 2010). It is unknown how much information people can and will handle in multiple behaviour change interventions. In this study, we investigated the level of completion of a web-based tailored intervention addressing five lifestyle behaviours (physical activity, fruit consumption, vegetable consumption, alcohol intake, and smoking), and tested potential information overload by comparing dropout rates for two versions of the programme, one offering a single behaviour change module as part of a sequential programme and one providing simultaneous tailored feedback on different behaviours. In addition, this study investigated personal predictors of dropout for the two versions of our computer-tailored programme. 


\section{METHODS}

\section{Design}

In this study, which was part of a randomized controlled trial (Dutch Trial Register NTR2168), tailored information was provided to two groups, one receiving a sequential behaviour tailoring condition (referred to below as sequential condition) and one receiving a simultaneous behaviour tailoring condition (simultaneous condition) (Schulz et al., 2011). The only difference between the conditions was that in the sequential condition, respondents were invited to focus their attention first on a single behaviour for which they failed to meet the Dutch national recommendations, whereas the simultaneous condition addressed all behaviours for which they failed to meet the Dutch recommendations at once. A detailed description of the study protocol has been published elsewhere (Schulz et al., 2011).

\section{Participants and procedure}

In the autumn of 2009, several Dutch Regional Health Authorities in the provinces of North-Brabant and Zeeland conducted an Adult Health Monitor study among adults (19-64 years) living in these provinces. This web-based questionnaire included demographics, aspects of general health and health-related topics. It also included questions regarding the respondents' lifestyle behaviours (physical activity, fruit and vegetable consumption, alcohol intake, and smoking). Completion took an average of 36 minutes $(S D=15.8)$. At the end of the questionnaire, respondents received information about the tailored programme. When interested in this programme, they could fill in their e-mail address. The eligibility criteria were participation in the Adult Health Monitor study, a valid e-mail address, and computer / Internet literacy. Approximately three weeks after completing the monitor questionnaire, participants interested in receiving tailored feedback received an e-mail enabling them to log on to the computer-tailored programme (see Figure 4.1). After approximately one month, people who did not respond to this e-mail received a reminder e-mail.

\section{Intervention}

The aim of the intervention was to stimulate participants to improve their lifestyle, focusing on five health behaviours. Based on the respondents' answers to the different questions, an expert system selected the appropriate feedback messages from a large database and presented these directly on the respondent's computer screen (Schulz et al., 2011). The I-Change model (De Vries et al., 2003) was used as a theoretical framework for the questionnaires and the tailored advice. 


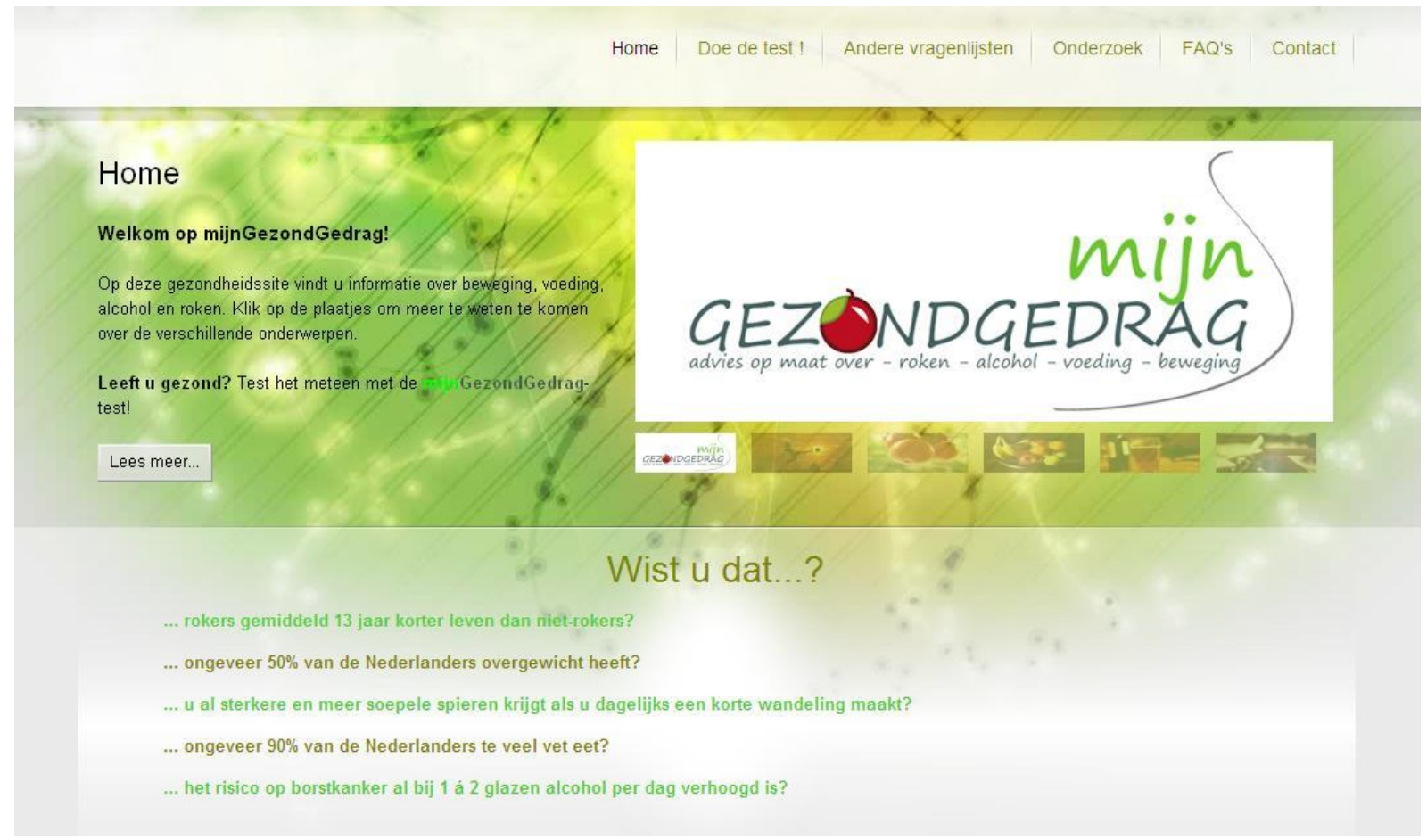

Figure 4.1 Screenshot of the homepage of the intervention programme

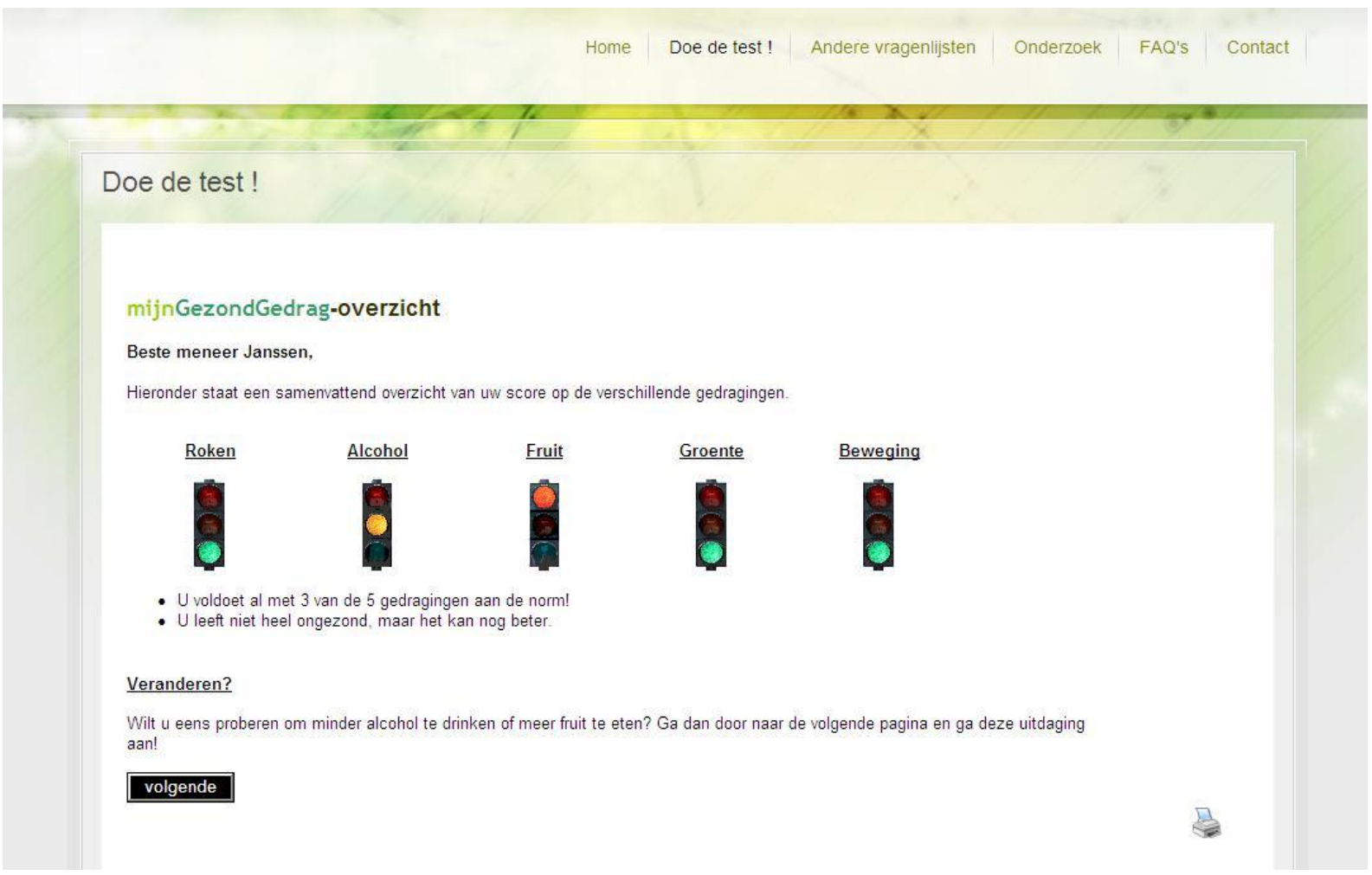

Figure 4.2 Screenshot of the health risk appraisal 


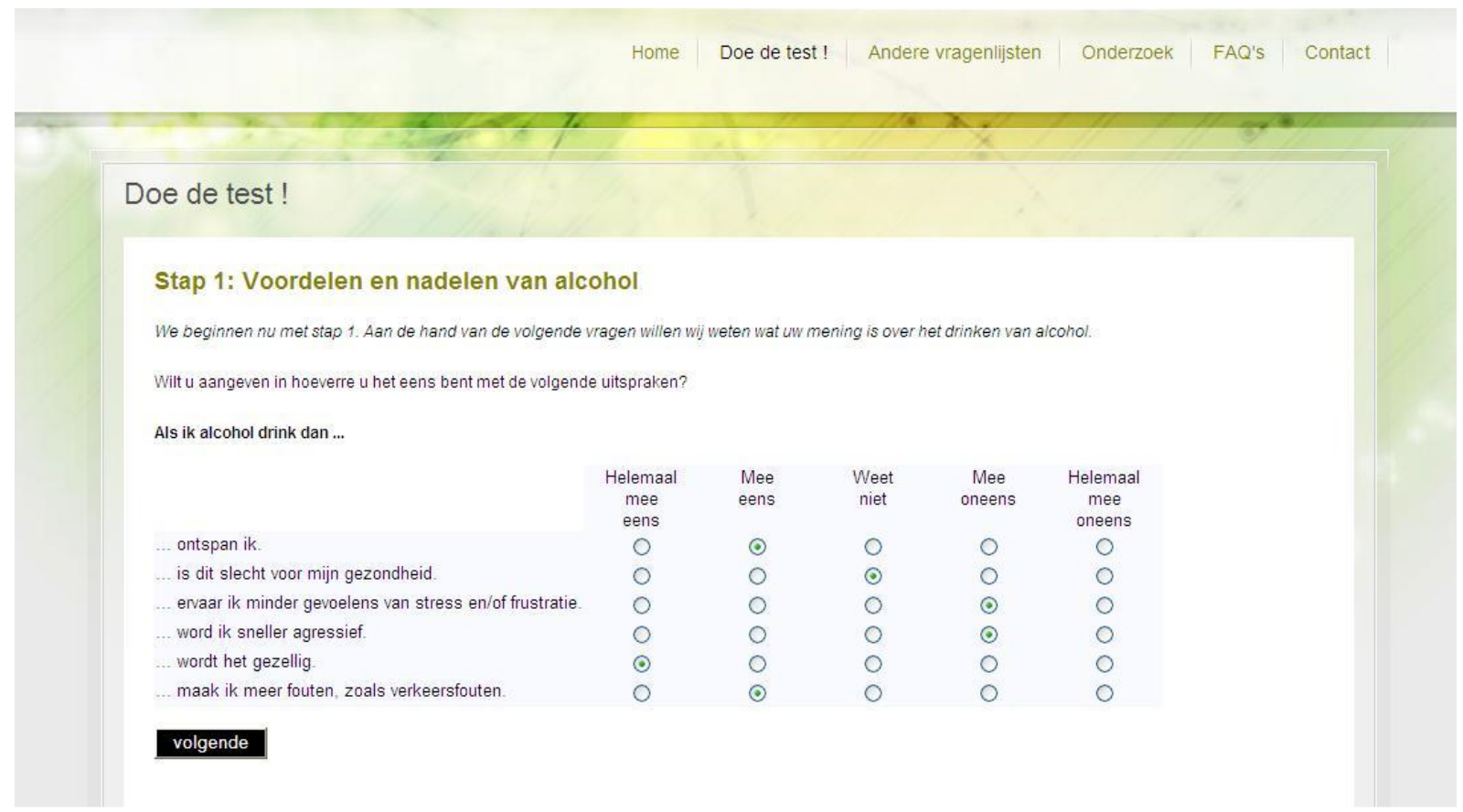

Figure 4.3 Screenshot of items regarding the pros and cons of alcohol intake

\section{Doe de test !}

Stap 1: De voordelen en nadelen van alcohol

Wat zijn voor u voordelen van alcohol?

Met een glaasje alcohol op voelt u zich meer ontspannen.

Een ontspannend gevoel is vaak het eerste effect van alcohol. Maar, alleen in kleine hoeveelheden ( 1 tot 2 glazen) werkt alcohol ontspannend. Wist $u$ dat alcohol bij meer dan 2 glazen juist angst- en spanningsverhogend werkt? U

Gezelligheid en alcohol gaan voor $\mathrm{u}$ hand in hand.

Alcohol werkt ontremmend. Daardoor durven mensen vaak meer te zeggen en meer te doen als ze een glaasje op zelfkritiek. Mensen kunnen dan dingen doen waar ze later spijt van krijgen: ruzle maken om niets, of zelfs (on) veilig vrijen met een wildvreemde. Overmatig alcoholgebrulk kan bovendien leiden tot agressief gedrag, niet ech gezellig.

Klik op 'volgende' om verder te gaan.

\section{volgende}

Figure 4.4 Screenshot of a personal advice regarding the pros of alcohol intake 


\section{Sequential condition}

After receiving the health risk appraisal, individuals in the sequential condition were invited to choose one of the health behaviours for which they were currently failing to meet the guideline. Respondents were encouraged to select the behaviour that they were most motivated to change. This was followed by a progressive scheme consisting of four steps, in which respondents received personal advice based on various psychosocial constructs: (1) attitude, (2) social influence, (3) preparatory plans, and (4) selfefficacy and coping plans regarding the lifestyle behaviour that they had chosen. Personal advice was given after the questions about each psychosocial construct (i.e. attitude questions were followed by personal feedback about these items).

\section{Simultaneous condition}

After receiving the health risk appraisal, participants in the simultaneous condition received feedback on all behaviours for which they failed to adhere to the public health guidelines in a predefined order. At random, half of the respondents started with the modules addressing preventive health behaviours (i.e. (1) physical activity, (2) vegetable consumption, (3) fruit consumption) and ended with the modules addressing addiction behaviours (i.e. (4) alcohol intake, (5) smoking), whereas the other half passed through the modules in reversed order. Respondents were presented with additional questions concerning psychosocial constructs, as well as personal advice on all behaviours for which they failed to adhere to the lifestyle recommendations. The four-step progressive scheme ((1) attitude, (2) social influence, (3) preparatory plans, and (4) self-efficacy and coping plans) was used for all relevant lifestyle behaviours. Again, questions and personal advice were presented alternately.

\section{Measures}

\section{Demographic information}

The following demographic variables were assessed: age, gender, educational level (no education, primary, or lower vocational school (low); secondary vocational school or high school (medium); or higher professional education or university (high)), income, current job status, marital status, number of persons in the household, and country of origin.

\section{Health status}

Quality of life was assessed using the SF-12 Health Survey (Ware et al., 1995; 1996). Symptoms of depression and anxiety were assessed by the Kessler Psychological Distress Scale (K10) (Wendel-Vos et al., 2003). Body mass index (BMI) was estimated from questions about height and weight. 


\section{Lifestyle behaviours}

Five lifestyle behaviours were assessed using validated questionnaires: (1) physical activity, (2) fruit consumption, (3) vegetable consumption, (4) alcohol intake, and (5) smoking. Physical activity was measured by the Short QUestionnaire to ASsess Health-enhancing physical activity (SQUASH) (Wendel-Vos et al., 2003), and guideline adherence was assessed using procedures developed by Ainsworth et al. (2000). Fruit consumption was measured using a four-item Food Frequency Questionnaire (FFQ) assessing weekly fruit and fruit juice intake (National Institute for Public Health and the Environment, 2005). Vegetable consumption was measured using a four-item FFQ assessing the weekly consumption of boiled or baked vegetables, as well as salads or raw vegetables (National Institute for Public Health and the Environment, 2005). Alcohol intake was measured by the five-item Dutch Quantity-Frequency-Variability (QFV) questionnaire (Lemmens et al., 1992). Smoking was assessed by asking participants if they smoked, what they smoked (cigarettes, cigars, or pipe tobacco), and how much they smoked per day (cigarettes) or per week (cigars or pipe tobacco).

\section{Psychosocial determinants}

The following description of the psychosocial determinants that were assessed is presented here to provide an overview of the programme; the data on these items were not included in the analysis. Based on earlier studies (Smeets et al., 2006), various psychosocial factors were assessed for the five different lifestyle behaviours: attitude (six items, such as "Eating 2 pieces of fruit every day is good for my health" - totally disagree to totally agree); social influence (three items, such as "How many people in your direct environment smoke?" - nobody to everybody); self-efficacy (six items, such as "I am able to eat sufficient vegetables when I have other delicious food at home" - no, definitely not to yes, definitely); preparatory plans (three items, such as "I intend to allow time for physical activity" no, definitely not to yes, definitely); and coping plans (six items, such as "I have made a plan to drink no more than two glasses of alcohol when I feel stressed or nervous" - totally disagree to totally agree).

\section{Programme use}

We counted the time respondents spent on the website during their first visit (i.e. from logging in to the programme until logging out or closing the website). Furthermore, we assessed the number of respondents who started with the first module and the number of respondents who filled out the programme completely. 
Table 4.1 Demographics, health status and lifestyle of the study sample $(N=3,473)$

\begin{tabular}{|c|c|}
\hline Variable & Total group \\
\hline Age $n=3,473$ & $43.61(19-64 ; S D=12.60)$ \\
\hline \multicolumn{2}{|l|}{ Gender $n=3,473$} \\
\hline Male & $1,849(53.2 \%)$ \\
\hline Female & $1,624(46.8 \%)$ \\
\hline \multicolumn{2}{|l|}{ Education $n=3,458$} \\
\hline Low & $367(10.6 \%)$ \\
\hline Medium & $1,607(46.5 \%)$ \\
\hline High & $1,483(42.9 \%)$ \\
\hline \multicolumn{2}{|l|}{ Income per month $n=3,468$} \\
\hline$<€ 1,000$ & $226(6.5 \%)$ \\
\hline$€ 1,001-€ 1,350$ & $228(6.6 \%)$ \\
\hline$€ 1,351-€ 1,750$ & $373(10.8 \%)$ \\
\hline$€ 1,750-€ 3,050$ & 1,177 (33.9\%) \\
\hline$>€ 3,051$ & $976(28.1 \%)$ \\
\hline "I don't want to say" & $488(14.1 \%)$ \\
\hline \multicolumn{2}{|l|}{ Employment situation $n=3,467$} \\
\hline Employed & $2,655(76.6 \%)$ \\
\hline Studying & $229(6.6 \%)$ \\
\hline Homemaker & $176(5.1 \%)$ \\
\hline Not currently in employment & $407(11.7 \%)$ \\
\hline \multicolumn{2}{|l|}{ Marital status $n=3,457$} \\
\hline Married & $2,092(60.5 \%)$ \\
\hline Living together & $528(15.3 \%)$ \\
\hline Unmarried & $639(18.5 \%)$ \\
\hline Divorced & $170(4.9 \%)$ \\
\hline Widowed & $28(0.8 \%)$ \\
\hline \# persons in household $n=3,473$ & $2.91(1-20 ; S D=1.42)$ \\
\hline \multicolumn{2}{|l|}{ Native country $n=3,471$} \\
\hline The Netherlands & $3,300(95.1 \%)$ \\
\hline Other & $171(4.9 \%)$ \\
\hline BMI $n=3,445$ & $25.17(15.03-58.11 ; S D=3.96)$ \\
\hline Quality of life $n=3,452$ & $40.11(16-48 ; S D=5.15)$ \\
\hline K10 (psychological distress) $n=3,461$ & $44.78(12-50 ; S D=5.70)$ \\
\hline \multicolumn{2}{|c|}{ Number of guidelines complied with $n=3,411$} \\
\hline 0 & $25(0.7 \%)$ \\
\hline 1 & $226(6.6 \%)$ \\
\hline 2 & $681(20.0 \%)$ \\
\hline 3 & $1,174(34.4 \%)$ \\
\hline 4 & $947(27.8 \%)$ \\
\hline 5 & $358(10.5 \%)$ \\
\hline \multicolumn{2}{|l|}{ Physical activity $n=3,473$} \\
\hline Compliance & $2,865(82.5 \%)$ \\
\hline Non-compliance & $608(17.4 \%)$ \\
\hline \multicolumn{2}{|l|}{ Vegetable consumption $n=3,446$} \\
\hline Compliance & $1,123(32.6 \%)$ \\
\hline Non-compliance & $2,323(67.4 \%)$ \\
\hline \multicolumn{2}{|l|}{ Fruit consumption $n=3,433$} \\
\hline Compliance & $1,560(45.4 \%)$ \\
\hline Non-compliance & $1,873(54.6 \%)$ \\
\hline \multicolumn{2}{|l|}{ Alcohol intake $n=3,473$} \\
\hline Compliance & $2,495(71.8 \%)$ \\
\hline Non-compliance & $978(28.2 \%)$ \\
\hline \multicolumn{2}{|l|}{ Smoking $n=3,473$} \\
\hline Compliance & $2,813(81.0 \%)$ \\
\hline Non-compliance & $660(19.0 \%)$ \\
\hline
\end{tabular}




\section{Statistical analyses}

The data was analysed using SPSS software, version 17.0. Descriptive statistics were used to describe the characteristics of the study sample and to calculate the dropout rates for the two tailoring conditions. In the sequential condition, a completer was defined as someone who filled in one module from start to finish (i.e. including the final question) since the aim of the first visit was that respondents of this condition complete one module relating to a lifestyle behaviour for which they failed to adhere to the guideline. In the simultaneous condition, a completer was defined as someone who completed all modules relating to the lifestyle behaviours for which they failed to adhere to the guidelines. The groups (i.e. completers versus non-completers) were compared in terms of their demographics and lifestyle behaviours by means of chi-square tests for discrete variables and independent-samples $t$ tests for continuous variables. In addition, effect sizes (ES) were calculated based on means (Cohen's d) and percentages (categorical variables). ES below 0.30 are considered small, while those between 0.30 and 0.80 are considered medium, and those larger than 0.80 are considered large (Cohen, 1988). Chi-square tests as well as ES calculations were also used to explore differences between the tailoring conditions in terms of their completion rates, based on the number of guidelines that respondents failed to meet. Logistic regression analyses, using the Enter method, were used to identify predictors of programme completion (demographics, health status, lifestyle behaviours and condition) within the entire sample. To identify interaction effects of tailoring condition and possible predictors, interaction terms were added to the regression equation. In the case of a significant interaction, logistic regression analyses were done separately for the two tailoring conditions to identify the predictors (demographics, health status, lifestyle behaviours).

\section{RESULTS}

\section{Participants' characteristics}

A total of 3,473 individuals participated in the present study. The mean age of the participants was 44 years. Slightly more men than women took part. With regard to the participants' lifestyle, $17.4 \%$ ( $n=608)$ failed to meet the physical activity guidelines, $67.4 \%(n=2,323)$ did not eat enough vegetables, $54.6 \%(n=1,873)$ did not eat enough fruit, $28.2 \%(n=978)$ drank too much alcohol, and $19.0 \%(n=660)$ reported that they smoked. Almost two-thirds did not adhere to two or more health behaviour guidelines $(n=2,106 ; 61.7 \%)$. The characteristics of the total sample are listed in Table 4.1.

\section{Completion and dropout}

As shown in Figure 4.5, the 3,473 people who logged on to the programme were evenly randomized to the two tailoring conditions. A total of 358 respondents $(10.3 \%)$ adhered to all five health guidelines. These people were not included in our further analyses, as no specific completion moment could be defined for this group. Of the remaining 3,115 respondents, 1,325 (42.5\%) logged out 
immediately after receiving the health risk appraisal, and 62 (2.0\%) even before receiving the health risk appraisal: in the sequential condition, $53.5 \%(n=821)$ started one lifestyle module, while in the simultaneous condition, $59.8 \%(n=907)$ started at least the first lifestyle module $\left(\chi^{2}{ }_{1}=12.48\right.$; $p<.001)$. Of the 821 starters in the sequential condition, 65.5\% ( $n=538)$ completed the module, while of the 907 starters in the simultaneous condition, $38.4 \%(n=348)$ completed the whole programme $\left(\chi^{2}=127.25 ; p<.001\right)$.

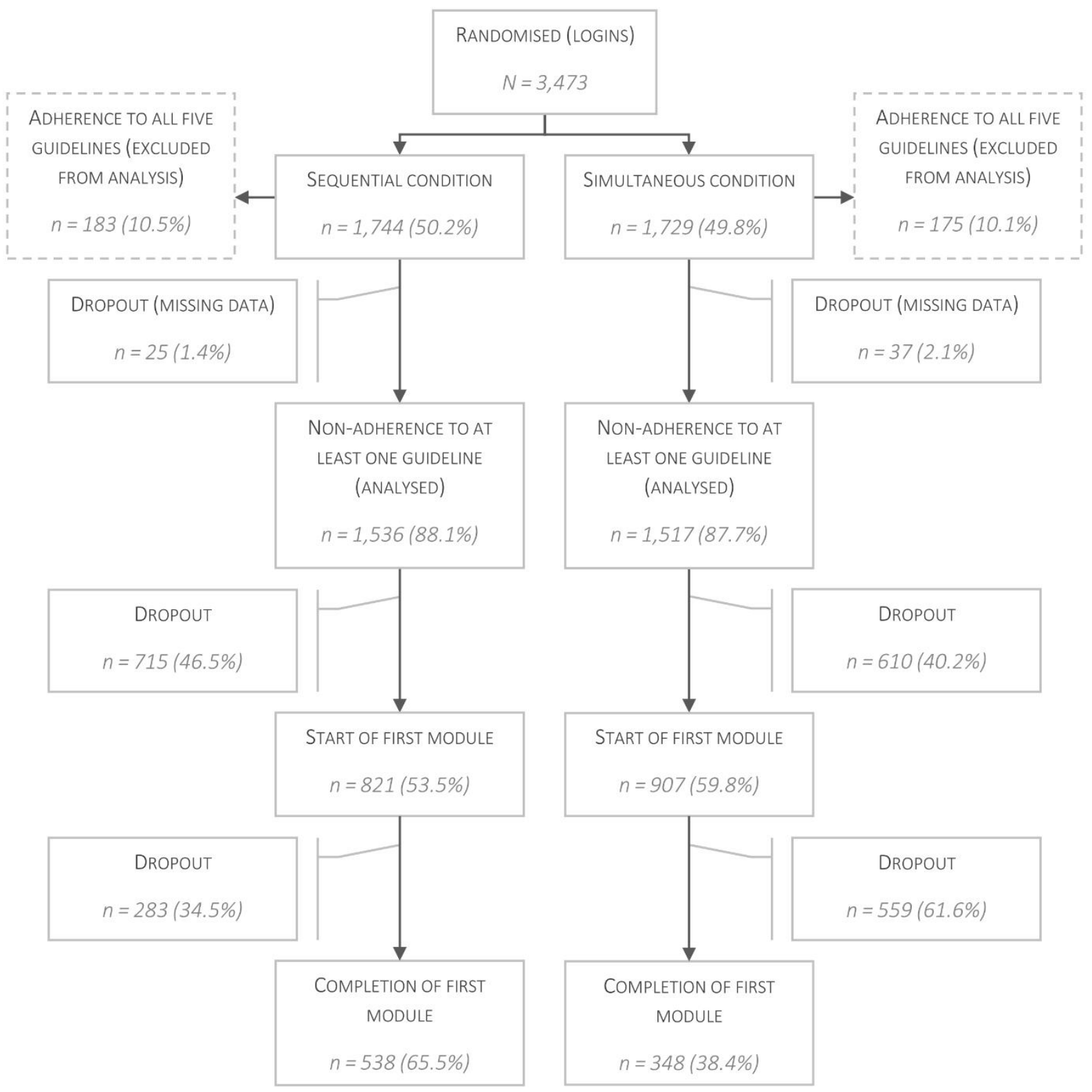

Figure 4.5 Attrition diagram

On average, respondents in the sequential condition spent 10 minutes and 8 seconds on the webbased tailored programme, while respondents in the simultaneous condition spent an average of 9 minutes and 47 seconds. In the sequential condition, respondents completed the programme on average within 18 minutes and 10 seconds, while non-completers spent an average of 6 minutes and 
20 seconds on the programme. In the simultaneous condition, respondents completed the programme within 20 minutes and 52 seconds, while non-completers left the programme on average after 6 minutes and 16 seconds.

\section{The influence of guideline adherence level on dropout}

The completion rate generally decreased as the number of guidelines that the respondents failed to meet increased (see Figure 4.6). However, this decline of the completion rates differed between the two conditions. Respondents in the simultaneous condition who failed to adhere to two or more guidelines were more likely to leave the site prematurely than those in the sequential condition who failed to adhere to the same number of guidelines.

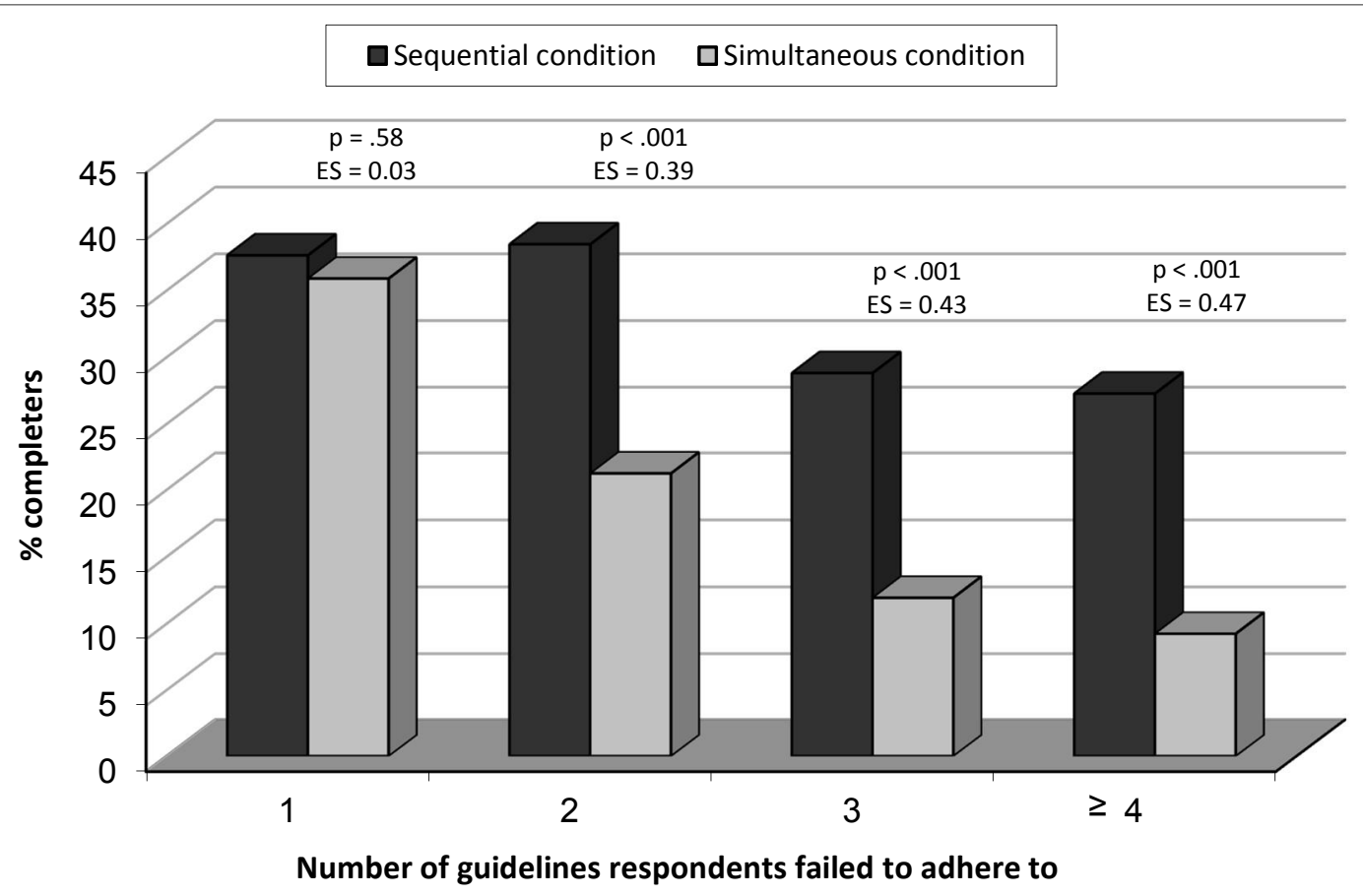

Figure 4.6 Number of guidelines respondents failed to adhere to against the number of completers in the sequential condition $(n=1,536)$ and the simultaneous condition $(n=1,517)$

\section{Differences between completers and non-completers}

The two tailoring groups did not differ in terms of their demographics, health status or lifestyle behaviours, indicating that randomization had been successful. A comparison of respondents who filled in the entire programme (i.e. completers) with respondents who prematurely left the site (i.e. non-completers) showed that the two groups differed on all variables, except for income, native country, K10 and alcohol intake (see Table 4.2). Medium ES regarding these differences concerned age and the number of guidelines respondents adhered to. Completers were older than non-completers were and completers adhered to more health guidelines than non-completers did. 
Table 4.2 Differences in demographics, health status and lifestyle between non-completers and completers ( $N=$ 3,053)

\begin{tabular}{|c|c|c|c|c|c|}
\hline Variable & $\begin{array}{r}\text { Non-completer } \\
n=2,167\end{array}$ & $\begin{array}{r}\text { Completers } \\
n=886\end{array}$ & $T(p)$ & $\chi^{2}(p)$ & ES \\
\hline Age & $41.85(S D=12.64)$ & $47.21(S D=11.96)$ & $11.07(<.001)$ & & 0.43 \\
\hline \multicolumn{6}{|l|}{ Gender } \\
\hline Male & $1,202(55.5 \%)$ & $450(50.8 \%)$ & & & \\
\hline Female & 965 (44.5\%) & $435(49.2 \%)$ & & $6.58(.04)$ & 0.09 \\
\hline \multicolumn{6}{|l|}{ Education } \\
\hline Low & $212(9.8 \%)$ & $120(13.6 \%)$ & & & \\
\hline Medium & 1,007 (46.7\%) & $424(48.0 \%)$ & & & \\
\hline High & $938(43.5 \%)$ & 339 (38.4\%) & & $13.35(.01)$ & 0.13 \\
\hline \multicolumn{6}{|l|}{ Income ${ }^{a}$ per month } \\
\hline$<€ 1,750$ & $504(23.3 \%)$ & $221(25.0 \%)$ & & & \\
\hline$€ 1,751-€ 3,050$ & $1,040(48.0 \%)$ & 437 (49.5\%) & & & \\
\hline$>€ 3,051$ & $621(28.7 \%)$ & $225(25.5 \%)$ & & $4.46(.35)$ & 0.08 \\
\hline \multicolumn{6}{|l|}{ Employment situation } \\
\hline$J o b$ & $1,852(85.6 \%)$ & $686(77.7 \%)$ & & & \\
\hline No job & $311(14.4 \%)$ & $197(22.3 \%)$ & & $33.34(.001)$ & 0.21 \\
\hline \multicolumn{6}{|l|}{ Relationship status } \\
\hline Single & $557(25.8 \%)$ & $191(21.8 \%)$ & & & \\
\hline In relationship & $1,602(74.2 \%)$ & $687(78.2 \%)$ & & $8.56(.01)$ & 0.11 \\
\hline \# persons in household & $2.99(\mathrm{SD}=1.45)$ & $2.74(\mathrm{SD}=1.25)$ & $-4.86(<.001)$ & & 0.18 \\
\hline \multicolumn{6}{|l|}{ Native country } \\
\hline The Netherlands & $2,075(95.8 \%)$ & $838(94.7 \%)$ & & & \\
\hline Other & $91(4.2 \%)$ & $46(5.3 \%)$ & & $1.52(.47)$ & 0.05 \\
\hline BMI & $25.00(\mathrm{SD}=3.93)$ & $25.54(S D=3.84)$ & $3.47(.001)$ & & 0.14 \\
\hline Quality of life & $40.19(S D=5.06)$ & $39.39(S D=5.64)$ & $-3.64(.001)$ & & 0.15 \\
\hline K10 & $44.71(S D=5.75)$ & $44.41(S D=6.12)$ & $-1.27(.203)$ & & 0.05 \\
\hline Adherence to guidelines & $2.83(S D=0.96)$ & $3.12(S D=0.88)$ & $8.22(<.001)$ & & 0.31 \\
\hline \multicolumn{6}{|l|}{ Physical activity } \\
\hline Compliance & $1,698(78.4 \%)$ & 761 (86.0\%) & & & \\
\hline Non-compliance & 469 (21.6\%) & $124(14.0 \%)$ & & $23.62(.001)$ & 0.18 \\
\hline \multicolumn{6}{|l|}{ Vegetable consumption } \\
\hline Compliance & 501 (23.1\%) & $253(28.6 \%)$ & & & \\
\hline Non-compliance & 1,666 (76.9\%) & 632 (71.4\%) & & $10.43(.005)$ & 0.12 \\
\hline \multicolumn{6}{|l|}{ Fruit consumption } \\
\hline Compliance & $801(37.0 \%)$ & $388(43.8 \%)$ & & & \\
\hline Non-compliance & $1,366(63.0 \%)$ & $497(56.2 \%)$ & & $13.14(.001)$ & 0.13 \\
\hline \multicolumn{6}{|l|}{ Alcohol intake } \\
\hline Compliance & $1,459(67.3 \%)$ & 632 (71.4\%) & & & \\
\hline Non-compliance & 708 (32.7\%) & $253(28.6 \%)$ & & $5.32(.07)$ & 0.08 \\
\hline \multicolumn{6}{|l|}{ Smoking } \\
\hline Compliance & $1,671(77.1 \%)$ & 731 (82.5\%) & & & \\
\hline Non-compliance & $496(22.9 \%)$ & $154(17.4 \%)$ & & $11.56(.003)$ & 0.12 \\
\hline \multicolumn{6}{|l|}{ Condition } \\
\hline Sequential & 998 (46.1\%) & $537(60.7 \%)$ & & & \\
\hline Simultaneous & 1,169 (53.9\%) & 348 (39.3\%) & & $54.74(.001)$ & 0.27 \\
\hline
\end{tabular}

Note: ${ }^{a}$ Respondents who did not want to report their income were classified in the category " $€ 1,751-€ 3,050$ ". 


\section{Predictors of programme completion}

We performed a logistic regression analysis to identify predictors of programme completion. After the various interaction terms had been added, the interaction term 'tailoring condition*non-adherence to guidelines' emerged as statistically significant $(B=-.620 ; p<.001)$ indicating that the effect of the number of guidelines respondents failed to adhere to on their completion status depended on the tailoring condition. Hence, separate analyses were performed for the two tailoring conditions. The results are presented in Table 4.3 (sequential condition) and Table 4.4 (simultaneous condition).

Table 4.3 Results of logistic regression analyses (Enter method) among the sequential condition on demographics and health status (model 1) and number of guidelines respondents failed to adhere to (model 2), with completion status (non-completers $=0$; completers $=1$ ) as dependent variable $(N=1,496)$

\begin{tabular}{|c|c|c|c|c|c|c|}
\hline \multirow[b]{2}{*}{ Variable } & \multicolumn{3}{|c|}{ Model 1} & \multicolumn{3}{|c|}{ Model 2} \\
\hline & OR & $p$ & $\mathrm{Cl}$ & OR & $p$ & $\mathrm{Cl}$ \\
\hline \multicolumn{7}{|l|}{ DEMOGRAPHICS } \\
\hline Age & 1.04 & $<.001$ & $1.02-1.05$ & 1.04 & $<.001$ & $1.02-1.05$ \\
\hline \multicolumn{7}{|l|}{ Gender } \\
\hline Male (reference) & 1.00 & & & 1.00 & & \\
\hline Female & 1.30 & .02 & $1.04-1.63$ & 1.27 & .04 & $1.01-1.59$ \\
\hline \multicolumn{7}{|l|}{ Education } \\
\hline Low & 1.42 & .99 & $0.98-2.05$ & 1.03 & .90 & $0.69-1.53$ \\
\hline Medium & 1.13 & .36 & $0.86-1.50$ & 1.14 & .30 & $0.89-1.45$ \\
\hline High (reference) & 1.00 & & & 1.00 & & \\
\hline \multicolumn{7}{|l|}{ Income a per month } \\
\hline$<€ 1,750$ & 1.42 & .06 & $0.98-2.05$ & 1.42 & .07 & $0.98-2.05$ \\
\hline$€ 1,751-€ 3,050$ & 1.13 & .38 & $0.86-1.50$ & 1.13 & .40 & $0.84-1.49$ \\
\hline$>€ 3,051$ (reference) & 1.00 & & & 1.00 & & \\
\hline \multicolumn{7}{|l|}{ Employment situation } \\
\hline Job (reference) & 1.00 & & & 1.00 & & \\
\hline No job & 1.01 & .96 & $0.74-1.38$ & 1.01 & .97 & $0.73-1.38$ \\
\hline \multicolumn{7}{|l|}{ Relationship status } \\
\hline In relationship (reference) & 1.00 & & & 1.00 & & \\
\hline Single & 0.94 & .71 & $0.68-1.30$ & 0.97 & .83 & $0.70-1.33$ \\
\hline \# persons in household & 0.94 & .18 & $0.87-1.03$ & 0.94 & .16 & $0.87-1.02$ \\
\hline \multicolumn{7}{|l|}{ Native country } \\
\hline The Netherlands (reference) & 1.00 & & & 1.00 & & \\
\hline Other & 0.99 & .93 & $0.57-1.66$ & 0.99 & .96 & $0.58-1.68$ \\
\hline \multicolumn{7}{|l|}{ HEALTH STATUS } \\
\hline BMI & 1.01 & .57 & $0.98-1.04$ & 1.01 & .62 & $0.98-1.04$ \\
\hline Quality of life & 0.97 & .07 & $0.94-1.00$ & 0.97 & .05 & $0.94-1.00$ \\
\hline K10 & 1.00 & .75 & $0.98-1.03$ & 1.00 & .78 & $0.98-1.04$ \\
\hline \multicolumn{7}{|l|}{ NON-ADHERENCE TO GUIDELINES } \\
\hline Number of guidelines & & & & 0.86 & .01 & $0.76-0.97$ \\
\hline Nagelkerke's $\mathrm{R}^{2}$ & & & .076 & & & .082 \\
\hline
\end{tabular}

Note: a Respondents who did not want to report their income were classified in the category " $€ 1,751-€ 3,050$ ". 
Table 4.4 Results of logistic regression analyses (Enter method) among the simultaneous condition on demographics and health status (model 1 ) and number of guidelines respondents failed to adhere to (model 2), with completion status (non-completers $=0$; completers $=1$ ) as dependent variable $(N=1,473$ )

\begin{tabular}{|c|c|c|c|c|c|c|}
\hline \multirow[b]{2}{*}{ Variable } & \multicolumn{3}{|c|}{ Model 1} & \multicolumn{3}{|c|}{ Model 2} \\
\hline & OR & $p$ & $\mathrm{Cl}$ & OR & $p$ & $\mathrm{Cl}$ \\
\hline \multicolumn{7}{|l|}{ DEMOGRAPHICS } \\
\hline Age & 1.04 & $<.001$ & $1.02-1.05$ & 1.04 & $<.001$ & $1.02-1.05$ \\
\hline \multicolumn{7}{|l|}{ Gender } \\
\hline Male (reference) & 1.00 & & & 1.00 & & \\
\hline Female & 1.35 & .03 & $1.04-1.74$ & 1.13 & .36 & $0.87-1.48$ \\
\hline \multicolumn{7}{|l|}{ Education } \\
\hline Low & 1.30 & .22 & $0.86-1.95$ & 1.41 & .11 & $0.92-2.16$ \\
\hline Medium & 1.02 & .88 & $0.77-1.35$ & 1.09 & .55 & $0.82-1.46$ \\
\hline High (reference) & 1.00 & & & 1.00 & & \\
\hline \multicolumn{7}{|l|}{ Income a per month } \\
\hline$<€ 1,750$ & 0.91 & .65 & $0.60-1.38$ & 0.87 & .51 & $0.56-1.33$ \\
\hline$€ 1,751-€ 3,050$ & 0.89 & .44 & $0.65-1.21$ & 0.90 & .51 & $0.65-1.24$ \\
\hline$>€ 3,051$ (reference) & 1.00 & & & 1.00 & & \\
\hline \multicolumn{7}{|l|}{ Employment situation } \\
\hline Job (reference) & 1.00 & & & 1.00 & & \\
\hline No job & 1.18 & .35 & $0.84-1.66$ & 1.11 & .56 & $0.78-1.58$ \\
\hline \multicolumn{7}{|l|}{ Relationship status } \\
\hline In relationship (reference) & 1.00 & & & 1.00 & & \\
\hline Single & 1.10 & .63 & $0.76-1.59$ & 1.12 & .55 & $0.77-1.65$ \\
\hline \# persons in household & 0.92 & .11 & $0.82-1.02$ & 0.90 & .06 & $0.81-1.01$ \\
\hline \multicolumn{7}{|l|}{ Native country } \\
\hline The Netherlands (reference) & 1.00 & & & 1.00 & & \\
\hline Other & 1.78 & .04 & $1.03-3.08$ & 1.54 & .14 & $0.87-2.70$ \\
\hline \multicolumn{7}{|l|}{ HEALTH STATUS } \\
\hline BMI & 1.00 & .79 & $0.96-1.03$ & 1.00 & .82 & $0.96-1.03$ \\
\hline Quality of life & 0.98 & .35 & $0.95-1.02$ & 0.98 & .18 & $0.94-1.01$ \\
\hline K10 & 1.00 & .82 & $0.97-1.04$ & 1.00 & .87 & $0.96-1.03$ \\
\hline \multicolumn{7}{|l|}{ NON-ADHERENCE TO GUIDELINES } \\
\hline Number of guidelines & & & & 0.49 & $<.001$ & $0.42-0.58$ \\
\hline Nagelkerke's $\mathrm{R}^{2}$ & & & .073 & & & .151 \\
\hline
\end{tabular}

In model 1 of both conditions, the factors significantly associated with non-completion were a lower age and being male. In the simultaneous condition, Dutch nationality was also significantly associated with dropout. In model 2, the effect of age remained significant in both conditions. In the sequential condition, being male continued to make a significant contribution, whereas in the simultaneous condition, the gender and native country variables became non-significant. In both conditions, discontinuation of the programme was predicted by the number of guidelines respondents failed to adhere to (in addition to a younger age). This means that people with a less healthy lifestyle were more likely to drop out than those with a healthier lifestyle. The second model of the sequential condition explained $8.2 \%$ of the total variance for programme completion, whereas the second model of the simultaneous condition explained $15.1 \%$ of the total variance. 


\section{DISCUSSION}

In view of the high number of people with an unhealthy lifestyle, there is a widely recognized need for interventions to change multiple behaviours. However, the best strategy to deliver such web-based interventions remains unclear. Addressing multiple health behaviours in one intervention leads to more extensive programmes, which require more time and effort from the respondents (e.g. Prochaska et al., 2008). We compared dropout rates of a sequential and a simultaneous version of a computer-tailored intervention regarding physical activity, fruit consumption, vegetable consumption, alcohol intake, and smoking, and investigated the predictive value of personal characteristics and lifestyle behaviours on completion and dropout rates for the two strategies.

Our first finding was that there were more non-completers in the simultaneous intervention than in the sequential intervention. The most important factor explaining the difference in dropout rate between these two conditions may be the difference in the length of the questionnaires and the computer-tailored advice that respondents received after the initial health risk appraisal. For example, if a respondent failed to adhere to two guidelines, the sequential intervention consisted of approximately 25 questions (average 10 minutes completion time), whereas the simultaneous intervention in such cases consisted of 50 questions, with an average completion time of 20 minutes. The advice also became twice as long, since the respondent had to fill in two modules in this case. Earlier research has also shown that the length of the programme may be a primary reason to leave a website prematurely (Brouwer et al., 2009). Another possible reason may be information overload (Prochaska, 2008). Since each psychosocial construct is measured and tailored for each relevant behaviour, this integrative approach is very demanding. An additional explanation that may need further research could be that in the simultaneous intervention, the five lifestyle modules had a predefined order, so respondents in this condition could not select the module they preferred to fill in first. Respondents in the sequential condition may have perceived more freedom of choice, since they could choose the lifestyle behaviour about which they wanted to receive personal feedback. This hypothesis could be tested in follow-up studies, including qualitative interviews.

Although the dropout rate was higher in the simultaneous intervention than in the sequential intervention, our findings revealed a high rate of non-completion in both types of intervention. One possible reason might be the recruitment strategy used. Completing the health risk appraisal took approximately five minutes in both conditions. The health risk appraisal was based on the Adult Health Monitor questionnaire that the respondents in our study had filled in at an earlier point in time. These respondents may not have wanted to make the effort of filling in a long questionnaire again. Hence, a considerable number of interested respondents of the potential target group may already have decided not to participate in the programme after receiving the health risk appraisal. A second possible reason for the high dropout rate might be the study sample. Our study sample consisted of people from the general population, who were primarily invited simply to fill in a health-related questionnaire. Our study might have shown different results in terms of dropout rates in a group that would have been included based on their motivation to learn something about their lifestyle and/or to change lifestyle behaviours. This means that lack of motivation to change lifestyle may have been a 
reason for dropping out in both conditions (Brouwer, 2011; Van 't Riet et al., 2010). Additionally, technical problems (Booth et al., 2008; Kypri \& McAnally, 2005), e.g. disruption of the Internet connection or errors on the website, as well as problems navigating through the website, could have played a role - as was suggested by several e-mails received from respondents.

In terms of personal characteristics that were predictive of completion or non-completion of the programme, significant influences were found of age and gender. Older people and women were more likely to complete the programme, which is in line with earlier findings (Brouwer et al., 2010). Furthermore, an unhealthy lifestyle was associated with higher dropout rates in both conditions. Earlier studies reported lower adherence to public health guidelines (i.e. an unhealthier lifestyle) among people with a low socioeconomic status compared to people with a higher socioeconomic status (Harris et al., 2011; Lampert, 2010), which means that this (high-risk) group in particular should be a target group for health promotion efforts. We found no difference in educational level, income, or employment status between respondents who dropped out at their first visit and those who did not. This is a relevant and promising finding, as it suggests that this tailored programme is equally accepted and appreciated by both groups.

Our findings - with dropout rates being higher in the simultaneous condition than in the sequential condition - suggest that a sequential tailoring strategy might be able to reach the largest group of participants. However, since approximately $60-70 \%$ of the population fails to adhere to multiple public health guidelines, people may need information about more than one lifestyle behaviour. The sequential strategy used in our intervention may therefore be insufficient to meet the needs of a large part of the population, especially those of people who are interested in several health behaviours and who are motivated to change multiple lifestyle behaviours. In our sequential intervention, respondents received the health risk appraisal, including information about the five health behaviours. Yet respondents were limited to one single module in the second part of the programme at their first visit. In the long term, this approach can be regarded as a multiple behaviour change intervention using a sequential strategy, but in the short term, detailed information is made available about one behaviour only. Since the dropout rate at the very first visit was high, future research should first concentrate on prolonged use (i.e. continuing the intervention for a substantial period of time) and possible information overload. To date, it seems to be a challenge to hold respondents' attention in online interventions. Since the dropout rate even in the sequential condition is rather high, the number of psychosocial constructs as well as the tailored texts could be shortened, spread over time or delivered in different forms. Including more interactive elements, such as videos or games, may improve the attractiveness of eHealth programmes, which in turn may result in longer visits (Severson et al., 2008; Stoddard et al., 2008; Vandelanotte \& Mummery, 2011). Stimulating re-visits, which are necessary in our sequential approach, poses a second challenge for future research.

The simultaneous tailoring strategy has advantages as well, insofar as people may receive tailored feedback on more than one lifestyle behaviour at once. However, it may be better not to offer the modules in a predefined order. A study by Brouwer et al. (2010) shows that when people have a 
choice to select more than one behaviour, they make use of this option and choose different behaviour modules.

Another option to explore is a mixture of both tailoring strategies, called preference-based tailoring (Kwak et al., 2007; Kwak et al., 2010) in which respondents can select the behaviour modules which they want to fill in (not limited to only one module). This may make respondents perceive a higher level of autonomy (Markland \& Vansteenkiste, 2007; Zuckerman et al., 1978) since they would not have to limit themselves to one single behaviour at first, and could receive as much information as they wish.

\section{Strengths, limitations and recommendations}

To our knowledge, this is the first study to compare sequential and simultaneous interventions addressing the five lifestyle behaviours of physical activity, fruit consumption, vegetable consumption, alcohol intake, and smoking, in terms of dropout rates. The study has yielded new information about predictors of completion of the two intervention types.

The findings of this study should be interpreted keeping several limitations in mind. Our findings were based on self-reports, which could have led to recall bias (e.g. the high proportion of people who reported to meet the physical activity guideline may represent an overestimation of their actual level of physical activity); and the amounts of variance explained by our regression models were relatively low, indicating that other factors might play a role in determining programme completion. Future research is necessary to identify additional relevant factors, for example, motivation to change, available time, interest in the topic, programme evaluation (in terms of, e.g., user-friendliness and attractiveness), and expectations from the programme.

The present study provides initial evidence for higher attrition rates in the simultaneous intervention strategy. Although this is likely to result in lower effectiveness of this intervention, future studies need to address the relative efficacy and effectiveness of simultaneous versus sequential tailoring. Hence, re-visiting rates for the two types of interventions should be compared, and the differences in effectiveness in terms of successful behaviour change should be tested. It is imaginable that despite the higher dropout in the simultaneous condition, more respondents in this condition received all relevant information compared to those in the single/sequential condition who possibly only read information about the most preferred behaviour module and/or never return to the intervention programme. More research remains to be done to study in which condition more modules are opened and/or completed by the respondents during the duration of the project.

\section{Conclusions}

Our findings indicate a high rate of non-completion in both types of intervention, with more incompletes in the simultaneous intervention and among respondents with unhealthier lifestyles. In 
both conditions, discontinuation of the programme was related to a younger age of the respondent, and in the sequential condition, being male was also associated with non-completion of the programme. The results of this study suggest opportunities for optimizing online tailored lifestyle interventions: such programmes should be tailored to all individual users; their efficiency should be improved; their attractiveness should be enhanced by integrating interactive elements; and their content and length or duration should be balanced. 


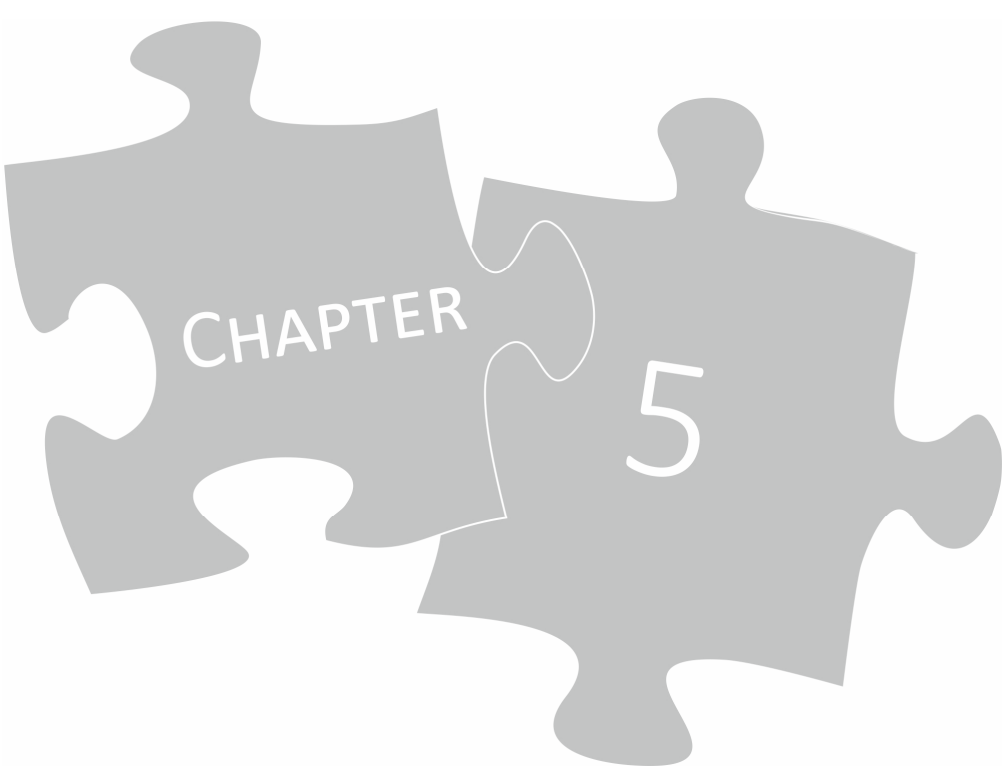

TAILORED EHEALTH LIFESTYLE PROMOTION: WHICH BEHAVIOURAL MODULES DO USERS PREFER?

This chapter has been accepted for publication as:

Schulz DN, Kremers SPJ, de Vries H. Tailored eHealth lifestyle promotion: Which behavioural modules do users prefer? Journal of Health Communication (in press). 


\section{ABSTRACT}

Health risk behaviours are widespread among adults and often co-occur. EHealth computer-tailored technology provides individuals with personalized feedback regarding multiple lifestyle behaviours. First, we investigated individuals' preferences for particular lifestyle modules, and hypothesised that health preventive behaviour modules would be preferred over addictive behaviour modules. Second, characteristics associated with these choices were examined. A web-based questionnaire assessed demographics, health status and five lifestyle behaviours (i.e. physical activity, fruit consumption, vegetable consumption, alcohol intake and tobacco use) among adults $(N=1,828)$. Responses were translated into a health risk appraisal outlining whether respondents adhered to the national guidelines for these behaviours. Next, respondents could select one of the lifestyle modules providing personalized advice. Over $60 \%$ of the participants failed to meet the guidelines for more than one lifestyle behaviour. The physical activity module was the most popular, followed by the smoking and fruit modules. Young adults tended to prefer the physical activity and fruit modules, whereas the vegetable module was more popular among older adults. No consistent pattern was identified for the alcohol and smoking modules. Our results support our hypothesis that health preventive behaviours, in particular physical activity, would be preferred. Although this could imply that physical activity could serve as a gateway behaviour when aiming at multiple behaviour changes, it is also conceivable that other mechanisms, such as the actual success of behaviour change, or the fact that people can choose, may increase chances of multiple behaviour change. Hence, mechanisms leading to multiple behaviour change need to be further explored.

Keywords: eHealth; user characteristics; computer-tailoring; multiple health behaviour change intervention; lifestyle modules 


\section{INTRODUCTION}

Physical inactivity, unhealthy diet, risky alcohol intake and tobacco use are key risk factors for chronic illness, and often co-occur (Ezzati et al., 2003). The following guidelines for different lifestyle behaviours have been defined in the Netherlands: being moderately physically active for 30 minutes during at least five days a week (Hildebrandt et al., 2010); eating 200 grams of vegetables a day (The Health Council of the Netherlands, 2006); eating two pieces of fruit a day (The Health Council of the Netherlands, 2006); not drinking more than one (women) or two (men) glasses of alcohol a day (The Health Council of the Netherlands, 2006); and not smoking (CBO, 2004). Nevertheless, previous studies revealed that a large proportion of Dutch adults do not meet the guidelines for one or more of these lifestyle behaviours (De Vries et al., 2008c; Robroek et al., 2010). It is especially men, younger adults, lower educated people, the economically inactive, those of lower social status, chronically ill people and singles who show lower adherence to multiple health behaviour guidelines (De Vries et al., 2008c; Poortinga, 2007; Pronk et al., 2004a). This implies that these risk groups have higher morbidity and mortality risks.

Promoting a healthy lifestyle by means of multiple behaviour change interventions is regarded as a promising approach to reduce the burden of disease (De Vries et al., 2008c; Prochaska et al., 2010). Computer-tailored lifestyle intervention programmes have an additional advantage in comparison to more traditional programmes in that they offer highly personalized feedback that is tailored to the needs of one particular person. They are therefore read and appreciated better, and have proved to be effective and to be able to reach large segments of the population via dissemination through e-mail or Internet, as well as being cost-effective (De Vries et al., 2008a; Krebs et al., 2010; Lustria et al., 2013; Oenema et al., 2008; Parekh et al., 2012; Smit et al., 2012a; Van Stralen et al., 2009a). These programmes involve personalized feedback based on the respondents' answers to questions that have been shown to predict healthy choices. Consequently, a respondent receives information that is personally relevant instead of general information regarding the topic in question. Previous research has shown that web-based interventions are more effective in reaching health behaviour change when making use of theory (Webb et al., 2010), especially the Theory of Planned Behaviour (Aijzen, 1991). The I-Change model (De Vries et al., 2003) - as used in our study - has proven to be an appropriate model in computer tailoring (Elfeddali et al., 2012a; Smit et al., 2012; Van Stralen et al., 2009). This model, which distinguishes three motivational phases, comprises different psychosocial theories, including the Theory of Planned Behaviour (Aijzen, 1991). By focussing on the different psychosocial concepts, this model can optimally be used when providing tailored advice.

Various studies on multiple behaviour change interventions recommend the use of a sequential strategy (Spring et al., 2004) and suggest allowing the individual to choose one or more behaviour modules (Campbell et al., 2000), instead of modules being presented in a predefined order. Offering respondents a choice of the behaviour they prefer to focus on may favourably influence success rates in terms of health behaviour change (Kremers et al., 2005). In order to inform intervention developers, it is important to examine the role of preferred and non-preferred lifestyle options. In particular, the results can be used to decide whether certain behaviours might serve as gateways to healthful 
changes in other behaviours (Dutton et al., 2008; Tucker \& Reicks, 2002; Wetzels et al., 2003). Yet, few studies have described which behaviours people like to start with and focus on (Brouwer et al., 2010; Campbell et al., 2000). A study by Brouwer et al. (2010) suggests that positive choices and preventive behaviours may be more appealing than negative choices, such as giving up unhealthy behaviours. We therefore hypothesized that health-promoting/preventive behaviours (i.e. physical activity, fruit and vegetable consumption) would be more often selected than addictive behaviours (i.e. alcohol intake and smoking).

Some studies have examined predictors of participating in, using and re-visiting eHealth programmes (Brouwer et al., 2010; Glasgow et al., 2007; Robroek et al., 2010; Schneider et al., 2012; Schulz et al., 2012b; Strecher et al., 2008; Van 't Riet et al., 2010). These studies generally found that women, older people, higher educated people, those with a higher level of income, and those with a healthy body mass index (BMI) and a healthier lifestyle are more likely to visit/start, use and/or revisit web-based interventions. Yet, these studies did not assess specific predictors of the choice from five different lifestyle behaviours.

In sum, this study had two objectives: (1) investigating users' preference for certain health behaviour modules in a web-based tailored behaviour change intervention and (2) assessing personal characteristics associated with the selection of certain modules, as specific subgroups might prefer to focus on different behaviours.

\section{METHODS}

\section{Design and ethical considerations}

This study was embedded in a randomized controlled trial (Dutch Trial Register NTR2168), which was approved by the Medical Ethics Committee of Maastricht University and the University Hospital Maastricht (MEC 09-3-016/NL27235.068.09). A detailed description of the study protocol has been published elsewhere (Schulz et al., 2011).

\section{Recruitment and inclusion criteria}

The recruitment took place via four Dutch Regional Health Authorities in North-Brabant and Zeeland, the Netherlands. These Regional Health Authorities had conducted the quadrennial Adult Health Monitor 2009 - a monitoring tool to assess general health and health-related topics - among inhabitants of the two provinces. In addition to a written version of this monitoring tool, an online version was available. Our intervention programme was integrated in this online version which means that participants, who completed the online version of the Monitor, were invited to participate in our study. An interface between the Adult Health Monitor and our intervention was created to allow transfer of data from the first system to the latter one. After giving informed consent and providing a 
valid e-mail address, interested participants were invited via e-mail to participate by logging in to our intervention by use of a personal login code and password. We used the following inclusion criteria: being 18 to 65 years old, having a computer with Internet access, basic Internet literacy (i.e. proven by completing the online Adult Health Monitor), and having a valid e-mail address.

\section{Web-based tailored intervention}

A multiple behaviour change intervention was developed aimed at improving five different lifestyle behaviours: increasing physical activity levels, increasing vegetable intake, increasing fruit intake, reducing overconsumption of alcohol and giving up tobacco use. Computer-tailoring was used to give personally relevant feedback messages (De Vries \& Brug, 1999; De Vries et al., 2008a). In a computertailored approach, all possible feedback messages are stored in a large database. An expert system selects the appropriate feedback messages on the basis of the respondents' answers to questionnaires. The appropriate messages are then presented on the respondent's computer screen.

Users of the intervention first received a health risk appraisal (HRA) indicating whether they were adhering to the following national public health guidelines: being moderately physically active for 30 minutes on at least five days a week; eating 200 grams of vegetables per day; eating two pieces of fruit per day; not drinking more than one (women) or two (men) glasses of alcohol a day; and not smoking. For each behaviour, they were shown a traffic light symbol indicating whether they met (green light), almost met (orange light) or failed to meet (red light) the guideline. Subsequently, the respondents could select one of the potentially five modules for which they had been given orange or red lights. Respondents who only failed to meet one guideline and thus received only one red or orange traffic light could not select a module, but automatically received personal advice regarding this one behaviour. Personal advice was provided in four steps based on questions about different psychosocial determinants of the I-Change model (De Vries et al., 2003): (1) attitude; (2) social influence; (3) preparatory planning; and (4) self-efficacy and coping planning. Personal advice was given at the end of each step.

\section{Questionnaire}

All questions used to generate the tailored messages were based on the I-Change model (De Vries et al., 2003) and derived from validated questionnaires or previous studies (De Vries et al., 2008a; Smeets, 2006).

Eight demographic variables were assessed: age, gender ( 1 = male; 2 = female), educational level ( 1 = low: no education, primary or lower vocational school; 2 = medium: secondary vocational school or pre-university school; 3 = high: higher professional education or university), income, current employment status $(1=$ employed; 2 = unemployed $/$ no job $)$, marital status $(1=$ single; 2 = in a 
relationship), number of persons in the household, and country of origin ( 1 = The Netherlands; 2 = other).

Health status was determined by assessing quality of life (SF-12 Health Survey) (Ware et al., 1995; 1996), symptoms of depression and anxiety (Kessler Psychological Distress Scale; K10) (Kessler \& Mroczek, 1994), and BMI calculated from questions about height and weight.

Lifestyle status was defined by assessing physical activity (Short QUestionnaire to ASsess Healthenhancing physical activity; SQUASH) (Wendel-Vos et al., 2003), fruit and vegetable consumption (four-item Food Frequency Questionnaires; FFQs) (National Institute for Public Health and the Environment, 2005), alcohol intake (five-item Dutch Quantity-Frequency-Variability (QFV) questionnaire) (Lemmens et al., 1992) and smoking (asking if they smoked, what they smoked (cigarettes, cigars, or pipe tobacco) and how much they smoked per day (cigarettes) or per week (cigars or pipe tobacco)). Based on the Dutch guidelines, we assessed whether the respondents complied with the five guidelines. A risk factor score was calculated by adding up the scores for all unhealthy behaviours. The value of the risk factor score could range from 0 to 5 .

Attitude was assessed by six items for each behaviour, asking respondents to indicate pros and cons of the lifestyle behaviour in question, e.g. that eating two pieces of fruit every day is good for one's health (range Cronbach's alpha $=.53$ to alpha $=.64$ ).

Social influence was assessed by three items for each behaviour about social modelling, social support and social norm, asking a respondent e.g. how many people in his or her direct environment smoked.

Preparatory planning was assessed by three items for each behaviour, asking respondents whether they had made particular plans preparing them for healthy behaviour, e.g. taking fruit to the work place (alpha $=.59$ to alpha $=.68$ ).

Self-efficacy was assessed by six items for each behaviour, asking whether a respondent felt able to behave in a healthy manner when in a difficult situation, e.g. feeling able to eat sufficient vegetables when one has other tempting food in the house (alpha $=.76$ to alpha $=.87$ ).

Coping planning was assessed by six items for each behaviour, asking whether respondents intended to make plans to cope with situations which they perceived as difficult, e.g. drinking no more than two glasses of alcohol when feeling stressed or nervous (alpha $=.89$ to alpha $=.94$ ). 


\section{Statistical analyses}

The data was analysed using IBM SPSS Statistics 19. Descriptive statistics were used to describe the characteristics of the study sample, and the flow of respondents from enrolment in the study to participation during the first visit and their choices regarding the different modules up to baseline completion of the programme. Chi-square tests were used to compare the completion rates for the five different modules.

The distribution of the modules selected by the respondents was summarized and analysed (objective 1 of the study). The distribution of the chosen modules was also assessed among different subgroups (defined on the basis of their risk factor score). Logistic regression analyses (backward method) were used to identify predictors of selecting (1) the physical activity module; (2) the vegetables module; (3) the fruits module; (4) the alcohol module; and (5) the smoking module (objective 2). The following variables were entered as independent variables in all regression models: gender, age, educational level, employment status, income, country of birth, marital status, BMI, psychological distress (K10), quality of life (SF-12), and risk factor score.

\section{RESULTS}

\section{Participants' characteristics}

Table 5.1 shows that our study sample consisted of 1,828 respondents with a mean age of 44 years and a mean BMl score of $25.2 \mathrm{~kg} / \mathrm{m}^{2} ; 51.4 \%$ were men. The majority had a medium or high educational level (89.9\%), had a monthly income of more than EUR 1,750 (60.7\%), were employed (75.8\%) and in a relationship (75.8\%). About $11.1 \%$ of the respondents complied with all five guidelines, $27.3 \%$ failed to meet the guideline for only one lifestyle behaviour, and the majority (61.6\%) failed to adhere to two or more guidelines. Non-adherence to the Dutch guidelines was most common for vegetable consumption (67.8\%) and fruit consumption (55.0\%), and least for smoking (18.5\%). 
Table 5.1 Demographics, health status and lifestyle of the study sample $(N=1,828)$

\begin{tabular}{|c|c|}
\hline Variable & Total group \\
\hline Age $n=1,828$ & $43.73(S D=12.68)$ \\
\hline \multicolumn{2}{|l|}{ Gender $n=1,828$} \\
\hline Male & $940(51.4 \%)$ \\
\hline Female & $888(48.6 \%)$ \\
\hline \multicolumn{2}{|l|}{ Education $n=1,761$} \\
\hline Low & $177(10.1 \%)$ \\
\hline Medium & $840(47.7 \%)$ \\
\hline High & $744(42.2 \%)$ \\
\hline \multicolumn{2}{|l|}{ Income per month a $n=1,767$} \\
\hline$<€ 1,750$ & $425(24.1 \%)$ \\
\hline$€ 1,750-€ 3,050$ & $860(48.7 \%)$ \\
\hline$>€ 3,050$ & $482(27.3 \%)$ \\
\hline \multicolumn{2}{|l|}{ Employment status $n=1,768$} \\
\hline Employed & $1,341(75.8 \%)$ \\
\hline Unemployed & $427(24.2 \%)$ \\
\hline \multicolumn{2}{|l|}{ Marital status $n=1,763$} \\
\hline In a relationship & $1,337(75.8 \%)$ \\
\hline Single & $426(24.2 \%)$ \\
\hline \# persons in household $n=1,770$ & $2.93(S D=1.48)$ \\
\hline \multicolumn{2}{|l|}{ Native country $n=1,768$} \\
\hline The Netherlands & $1,676(94.8 \%)$ \\
\hline Other & $92(5.2 \%)$ \\
\hline $\mathrm{BMI} n=1,816$ & $25.24(\mathrm{SD}=3.99)$ \\
\hline Quality of life ${ }^{b} n=1,754$ & $40.03(S D=5.25)$ \\
\hline K10 (psychological distress) ${ }^{\mathrm{c}} n=1,761$ & $44.69(S D=5.85)$ \\
\hline \multicolumn{2}{|l|}{ Number of risk factors $n=1,736$} \\
\hline 0 & $192(11.1 \%)$ \\
\hline 1 & $474(27.3 \%)$ \\
\hline 2 & $592(34.1 \%)$ \\
\hline 3 & $351(20.2 \%)$ \\
\hline 4 & $114(6.6 \%)$ \\
\hline 5 & $13(0.7 \%)$ \\
\hline \multicolumn{2}{|l|}{ Physical activity $n=1,736$} \\
\hline Compliance & $1,445(83.2 \%)$ \\
\hline Non-compliance & $291(16.8 \%)$ \\
\hline \multicolumn{2}{|l|}{ Vegetable consumption $n=1,736$} \\
\hline Compliance & $559(32.2 \%)$ \\
\hline Non-compliance & $1,177(67.8 \%)$ \\
\hline \multicolumn{2}{|l|}{ Fruit consumption $n=1,736$} \\
\hline Compliance & $781(45.0 \%)$ \\
\hline Non-compliance & 955 (55.0\%) \\
\hline \multicolumn{2}{|l|}{ Alcohol intake $n=1,736$} \\
\hline Compliance & $1,248(71.9 \%)$ \\
\hline Non-compliance & $488(28.1 \%)$ \\
\hline \multicolumn{2}{|l|}{ Smoking $n=1,736$} \\
\hline Compliance & $1,415(81.5 \%)$ \\
\hline Non-compliance & $321(18.5 \%)$ \\
\hline
\end{tabular}

Note: ${ }^{a}$ Respondents who did not want to report their income $(n=269)$ were classified in the category " $€ 1,751$ - $€$ 3,050";

${ }^{b}$ Higher scores indicate a higher quality of life; ${ }^{c}$ Higher scores indicate less psychological distress.

\section{Module selection}

Figure 5.1 shows the flow of respondents from enrolment in the study to participation during the first visit and their choices regarding the different modules up to baseline completion of the programme. 


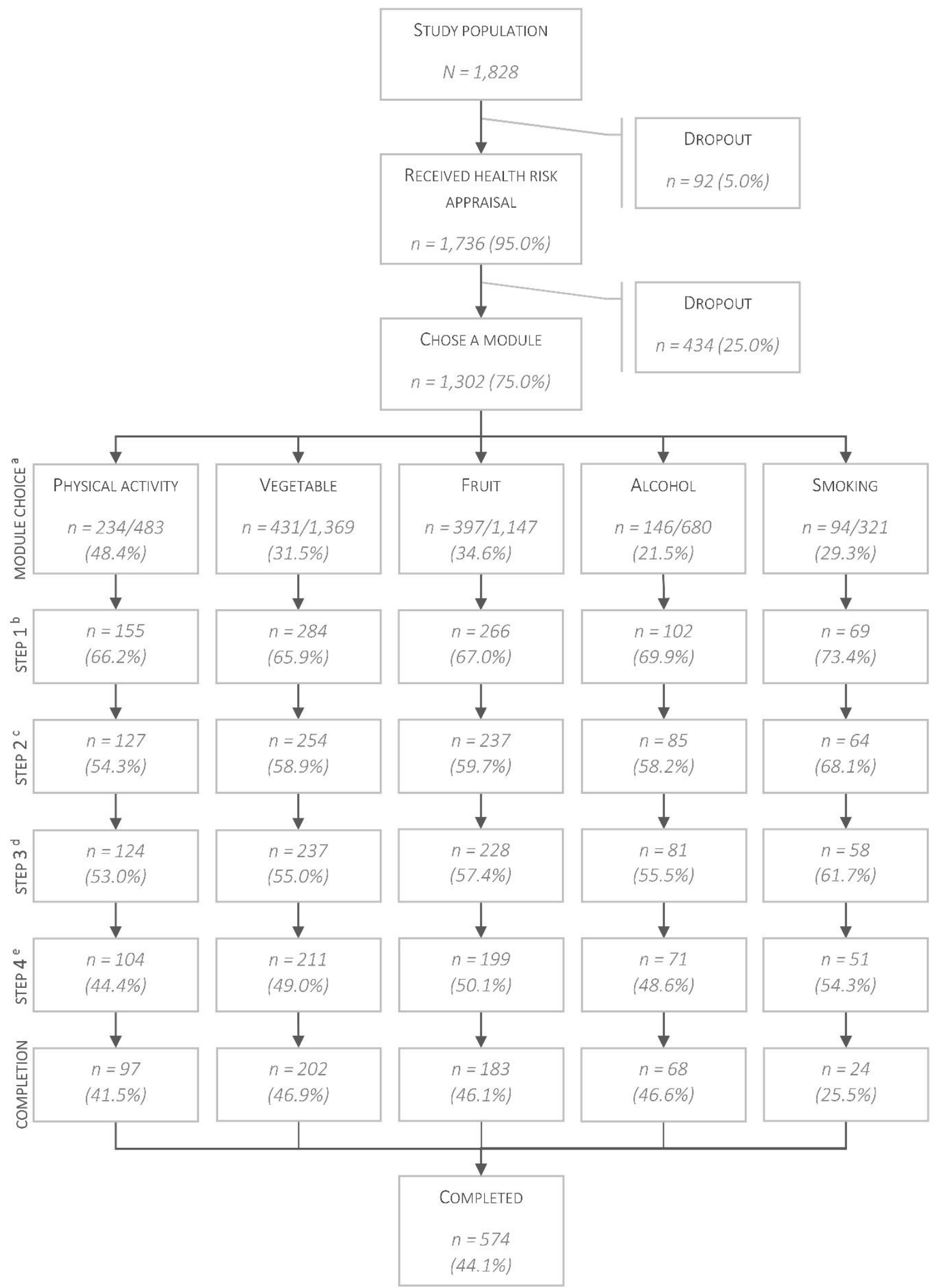

Figure 5.1 Flow-chart

Note: a Number of respondents choosing the particular module, out of the total number of respondents not meeting the guideline in question or meeting all five guidelines; ${ }^{b}$ Attitude; ${ }^{c}$ Social influence; d Preparatory planning; ${ }^{e}$ Self-efficacy and coping planning. 
Of the 1,736 respondents who received the HRA, one quarter disengaged from the programme and three quarters selected a lifestyle module. Of the respondents who received the HRA, 1,036 (59.7\%) failed to comply with two or more guidelines and thus had the possibility to choose a particular module to start with; 782 (75.5\%) of them actually selected a module. In total, 476 respondents (27.9\%) did not have the possibility to select a module, since they only failed to meet one guideline, and were thus advised to use the corresponding module. A total of $350(73.5 \%)$ of these respondents continued to use the relevant module. Additionally, 192 respondents complied with all five guidelines. Nevertheless, three quarters of them $(n=144 ; 73.5 \%)$ did select a module, almost $80 \%$ of them selecting the physical activity module $(n=114 ; 79.2 \%)$.

The results depicted in Figure 5.1 support our hypothesis that preventive behaviours would be chosen more often than addictive behaviours. Of the 483 respondents who were eligible to choose the physical activity module, $234(48.4 \%)$ actually chose this module. The corresponding figures for fruit consumption were $n=397$ out of 1,147 (34.6\%) and those for vegetable consumption $n=431$ out of 1,369 (31.5\%). Table 5.2 shows the results of a more detailed test of our hypothesis.

A total of 574 respondents (44.1\%) completed the module they had chosen. Figure 5.1 also clearly shows that the completion rate was significantly lower for the smoking module than for the other modules (physical activity: $\chi^{2}=7.37, p=.007$; vegetables: $\chi^{2}=15.17, p<.001$; fruit: $\chi^{2}=12.99$, $p<.001$; alcohol: $\left.\chi^{2}=10.74, p=.001\right)$.

\section{Distribution of selections}

To further investigate findings regarding our hypothesis concerning preference for preventive behaviours, Table 5.2 presents the distribution of the selection of different modules in relation to the risk factor score and the total distribution per module. The numbers corresponding to the risk factors are listed in column 1. Column 2 presents the real chance that a particular module is chosen. The other columns present the percentages of people who chose a particular module in relation to the number of risk factors (and thus to the possibility to choose a particular module).

The results for the respondents who reported adhering to all five guidelines (risk factor score $=0$ ) show that the physical activity module was selected by $78.9 \%$ of this group of respondents. This means that this module was selected 3.16 times more often than based on chance, and relatively more often than the vegetable, fruit and alcohol modules.

Respondents who failed to comply with two or three guidelines (risk factor score $=2$ or 3 ) were most likely to select the physical activity module (as indicated by the ratios, which are significantly greater than 1), followed by the fruit module and the smoking module. Among all subgroups, the vegetable module was least likely to be selected.

As the last two rows of Table 5.2 show, the physical activity module was selected by $65.3 \%$ of the respondents who were eligible to choose this module (ratio $=2.24$ ). The vegetable module and the 
alcohol module were selected by $28.5 \%$ (ratio $=0.74$ ) and $21.4 \%$ (ratio $=0.64$ ), respectively, of the respondents who were eligible to choose these modules. The actual selection distributions of the fruit and smoking modules were similar to the real chance of selecting fruit and smoking, respectively.

Table 5.2 Probability distribution

\begin{tabular}{|c|c|c|c|c|c|c|}
\hline $\begin{array}{l}\text { Number of risk } \\
\text { factors } \\
\text { (red/orange } \\
\text { traffic lights) }\end{array}$ & $\begin{array}{r}\text { Chance } \\
\text { per } \\
\text { module }\end{array}$ & $\begin{array}{r}\text { Physical activity } \\
\text { module }\end{array}$ & $\begin{array}{r}\text { Vegetable } \\
\text { module }\end{array}$ & $\begin{array}{r}\text { Fruit } \\
\text { module }\end{array}$ & $\begin{array}{l}\text { Alcohol } \\
\text { module }\end{array}$ & $\begin{array}{r}\text { Smoking } \\
\text { module }\end{array}$ \\
\hline 0 & $25.0 \%$ & $78.9 \%\left(3.16^{*}\right)$ & $7.0 \%(0.28 *)$ & $7.7 \%\left(0.31^{*}\right)$ & $6.3 \%\left(0.25^{*}\right)$ & -- \\
\hline 1 & $100.0 \%$ & $100.0 \%(1.00)$ & $100.0 \%(1.00)$ & $100.0 \%(1.00)$ & $100.0 \%(1.00)$ & $100.0 \%(1.00)$ \\
\hline 2 & $50.0 \%$ & $68.8 \%(1.38 *)$ & $42.3 \%\left(0.85^{*}\right)$ & $62.2 \%\left(1.24^{*}\right)$ & $30.1 \%(0.60 *)$ & $44.9 \%(0.90)$ \\
\hline 3 & $33.3 \%$ & $53.5 \%(1.61 *)$ & $25.1 \%\left(0.75^{*}\right)$ & $35.4 \%(1.06)$ & $28.9 \%(0.87)$ & $40.4 \%(1.21)$ \\
\hline 4 & $25.0 \%$ & $40.0 \%(1.60)$ & $14.7 \%(0.59 *)$ & $25.7 \%(1.03)$ & $25.5 \%(1.02)$ & $26.6 \%(1.06)$ \\
\hline 5 & $20.0 \%$ & $33.3 \%(1.67)$ & $0.0 \%(0.00 *)$ & $22.2 \%(1.11)$ & $11.1 \%(0.56)$ & $33.3 \%(1.67)$ \\
\hline Real chance ${ }^{a}$ & & $31.6 \%$ & $38.5 \%$ & $38.1 \%$ & $33.7 \%$ & $34.0 \%$ \\
\hline $\begin{array}{l}\text { Actual } \\
\text { distribution }\end{array}$ & & $65.3 \%(2.24 *)$ & $28.5 \%\left(0.74^{*}\right)$ & $40.9 \%$ (1.07) & $21.4 \%(0.64 *)$ & $37.0 \%(1.09)$ \\
\hline
\end{tabular}

\section{Predictors of selecting each of the modules}

Regression analyses were used to assess personal characteristics associated with the selection of each of the five different modules (our second study aim). Table 5.3 shows that a consistent pattern was found with regard to the health-promoting behaviours (i.e. physical activity, fruit consumption and vegetable consumption): selection of these modules was predicted by a healthier lifestyle. An inconsistent pattern regarding these behaviours was found for age. Whereas lower age was associated with selecting the physical activity module and the fruit module, higher age was significantly associated with selecting the vegetable module.

No similarity was found between the predictors of selecting the alcohol and smoking modules. Selecting the alcohol module was predicted by being male, having a lower income and having higher levels of psychosocial distress. Educational level, quality of life and psychological distress significantly predicted whether the smoking module would be selected. Compared to higher educated people, respondents with a medium level of education were more likely to select the smoking module. A lower level of psychological distress and a lower quality of life were also associated with selecting the smoking module. 


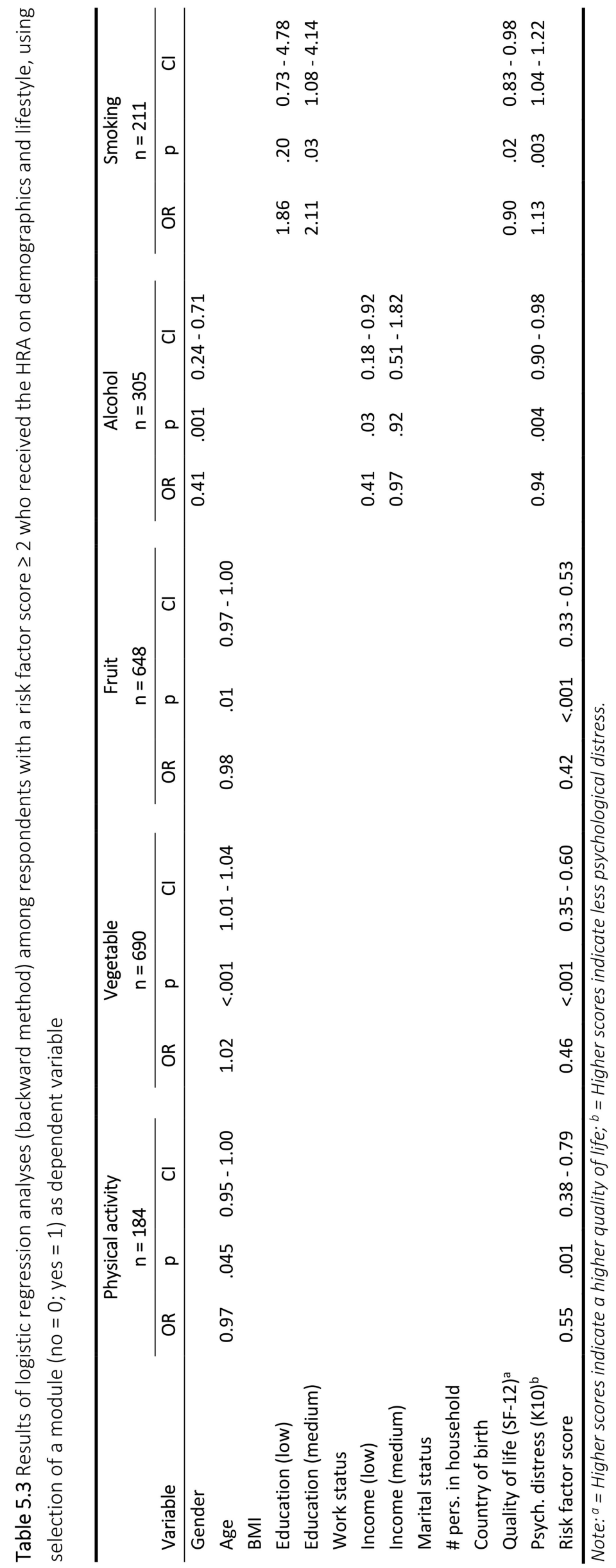




\section{DISCUSSION}

In our web-based tailored eHealth lifestyle intervention programme targeting adults, respondents were stimulated to increase their physical activity levels, improve their nutrition by eating more fruit and vegetables, limit their alcohol intake to one or two glasses per day, and/or to give up tobacco use. In this study, we first assessed the respondents' preferences for different lifestyle modules integrated in the multiple behaviour change intervention. In a second step, we assessed user characteristics associated with their preference for the modules. The main finding was that the majority of respondents preferred to choose the physical activity module, especially the younger respondents and those with a healthier lifestyle in general.

Our findings are consistent with those of a previous study, which found that most of their participants started with the physical activity module (80\%) rather than a featured fat module (69\%) and a smoking module (68\% of smokers) (Brouwer et al., 2010). Our fruit module was also selected more often than the vegetable and alcohol modules, but approximately as frequently as the smoking module. An earlier study by Campbell and colleagues (2000) found that the majority of participants chose healthy eating and/or physical activity when asked to prioritize the behaviour they most wanted to change (out of smoking, physical activity, nutrition, and cancer screening). Their study, however, involved only women. In our study, the vegetable module was chosen relatively infrequently, but it appeared to have been selected especially by the older respondents. All three health-promoting modules (physical activity, fruit and vegetables) were more often selected by people with a healthier lifestyle. This finding is consistent with findings regarding exposure, use and re-visits regarding web-based lifestyle interventions (Brouwer et al., 2010; Verheijden et al., 2007). Gender, socioeconomic status, marital status and health status were not found to be related to the preference for one of the healthpromoting behaviour modules. The alcohol module was more often selected by men, by lower income groups and by people with higher levels of psychological distress. In general, Dutch men have been found to consume more alcohol than women (Geels et al., 2013). The current intervention succeeded in reaching (in terms of the alcohol module being selected) those people who may benefit most from the alcohol module (i.e. risk groups like men, lower income groups and people experiencing psychological distress). The smoking module was preferred by people with medium educational levels, a lower quality of life and lower levels of psychological distress. Our study has thus yielded new information regarding personal characteristics of people who prefer to address different kinds of lifestyle behaviours in an eHealth intervention.

More than $60 \%$ of the participants failed to meet more than one of the guidelines defined for physical activity, fruit consumption, vegetable consumption, alcohol intake and smoking, indicating that multiple behaviour change interventions are important. Previous studies have shown that certain behaviours tend to cluster, especially energy balance-related behaviours (i.e. physical activity and diet), and that synergistic effects might occur in health promotion programmes (Berrigan et al., 2003; Johnson et al., 1998; Kremers et al., 2004; Schuit et al., 2002). Thus, successful change in one behaviour may lead to changes in another, clustered behaviour. For the development of future lifestyle interventions, it is important to know that the physical activity module was the most popular 
among our study population. In view of its popularity, it is conceivable that the use of physical activity modules may also motivate people to change other behaviours as well. However, this hypothesis needs to be explored further. It is also conceivable that it is not necessarily the type of behaviour which may lead to addressing other behaviours, but the chances of successful behaviour change, or the fact that respondents had a choice. In a multiple behaviour change intervention, the physical activity module could be linked to the other health behaviour modules, e.g. by integrating information about a healthy lifestyle in general or by including specific information regarding healthy nutrition or non-smoking.

Regarding fruit and vegetable intake, it has been reported that only a small part of the adult population adhere to the guidelines (De Vries et al., 2008c; Van Rossum et al., 2011), which is consistent with the findings of this study. Hence, improvements in nutrition behaviour seem to be particularly important, and an intervention should be successful in getting people to change their nutrition behaviour. In our intervention, however, the vegetable module was selected relatively seldom. This implies that different lifestyle behaviours may need different kinds of interventions.

The present multiple behaviour change intervention appeared to be successful in reaching some highrisks groups (i.e. men, lower educated people, lower income groups and people experiencing psychological distress). However, completion rates of the modules were quite low; the smoking module in particular was completed significantly less often than the other modules. Previous studies have also shown that dropout rates in Internet interventions are generally high (A.W. Hansen et al., 2012; Leslie et al., 2005; Verheijden et al., 2007). This implies that incompletion during the first visit is common, as is dropout over time (Brouwer et al., 2010; Verheijden et al., 2007). Hence, it is important to improve the participation rates and use of eHealth websites. Engagement is a prerequisite for participation. This means that users should be involved in health content in ways that motivate and lead to health behaviour change (Lefebvre et al., 2010), which could prevent disengagement from the programme. Attrition rates from web-based tailored interventions appear to be higher among low socioeconomic status groups (Bennett \& Glasgow, 2009; Eysenbach, 2005), men and younger people (Schulz et al., 2012b; Strecher et al., 2008). Moreover, groups with an overall unhealthier lifestyle are more likely to drop out (Schulz et al., 2012b). Thus, high-risks groups should be the target group for multiple behaviour change interventions. It is important to optimize the programmes in such a way as to make them attractive to these groups (Brouwer et al., 2010; Vandelanotte et al., 2013; Van 't Riet et al., 2010).

Approximately $10 \%$ of the respondents of our study were characterized by a healthy lifestyle (i.e. adhering to all five guidelines). However, three quarters of them nevertheless selected a module, the majority selecting the physical activity module. For this population it is important to maintain a healthy lifestyle, and this may present an extra challenge for eHealth interventions, in addition to focusing on individuals with an unhealthy lifestyle. 


\section{Strengths and limitations}

To our knowledge, this is the first study to investigate people's preference when offered a choice between five different lifestyle behaviours in a tailored eHealth lifestyle programme. The study, which used a large sample, was also successful in yielding new information regarding the personal characteristics of people who select different kinds of lifestyle behaviours as the first one to address. In our intervention, the modules were delivered sequentially over time, which means that respondents could only choose one lifestyle module in the first year (Schulz et al., 2011). Future studies could explore which and how many modules would be chosen if participants had the possibility to select more than one behavioural module at a time.

Our study had the following limitations. First, the results of this study were based on self-reports, and inaccurate self-reporting might have occurred through social desirability bias, recall bias (under- or over-estimation), or errors in self-observation due to factors like cognitive bias or memory lapses. For example, the high percentage of respondents that reported adhering to the physical activity guideline may be an indication of recall bias by overestimating the actual amount of physical activity, since this percentage is not in line with previous findings for Dutch adults (Netherlands Institute for Sport \& Physical Activity, 2013; Smeets, 2006). Second, the study suffered from high dropout rates. Around $25 \%$ of respondents dropped out after receiving the HRA and another 56\% dropped out while going through one of the behaviour modules. Dropout increased after each session. This implies that more research is needed to identify which particular topics are crucial for respondents, and whether particular sessions should also be tailored to the needs and characteristics of the participants. For instance, we provided tailored feedback on the attitudes to respondents who were already motivated to change. Although aimed at reinforcing attitudes and providing positive feedback, this strategy may also have increased the risk of dropout. Since most respondents were motivated, this information may have been redundant (Dijkstra et al., 1998b; Velicer et al., 1999a). Thus, more research is needed to foster engagement in programmes to reduce programme dropout.

\section{Conclusion}

The most popular module in our tailored lifestyle intervention was the physical activity module. The results suggest that younger people and those with a healthier lifestyle were most likely to start with the physical activity or fruit module; the vegetable module was especially chosen by older respondents and by those with a healthier lifestyle; the alcohol module was preferred by men, those with a lower income and those experiencing more psychological distress; and the smoking module was particularly selected by people with a medium level of education, less psychological distress and a lower quality of life. In general, our intervention succeeded in reaching certain high-risk groups, such as men, lower socioeconomic status groups and people suffering from psychological distress. As suggested by Campbell et al. (2000), web-based computer-tailoring may be a promising approach to let respondents self-select the behaviour they prefer to focus on. The results give a preliminary indication that physical activity may serve as a gateway behaviour when aiming at multiple behaviour 
CHAPTER 5

change in tailored eHealth lifestyle promotion. Additional research is essential to further optimize programme engagement. 


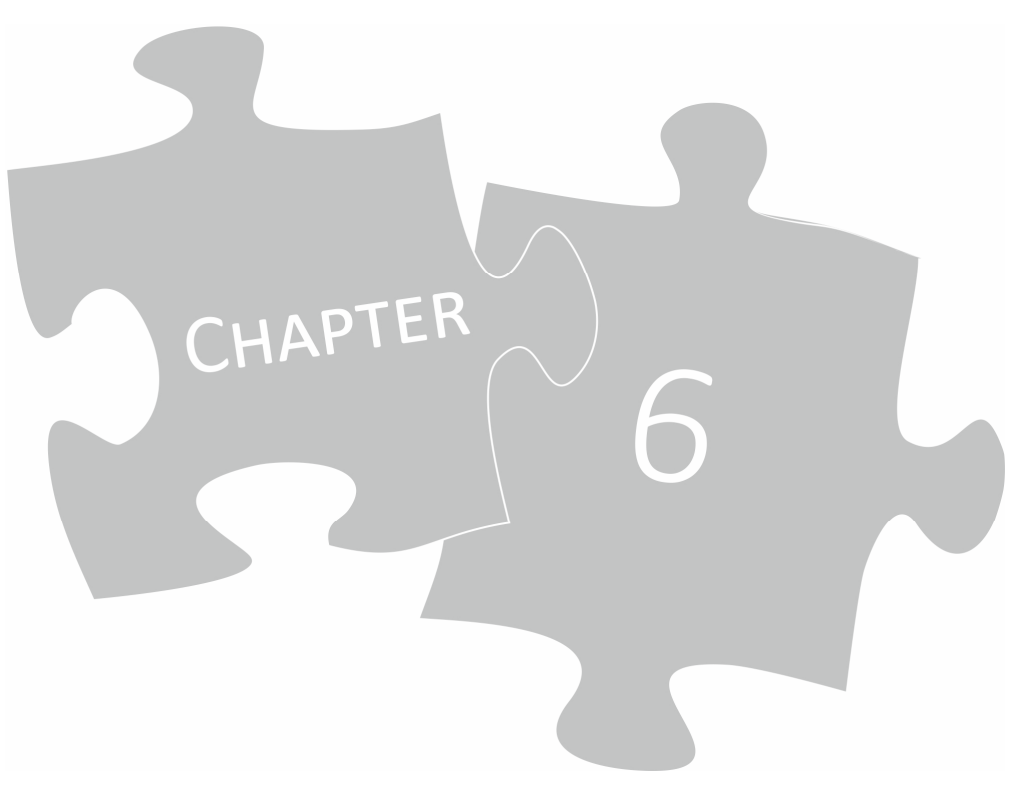

EFFECTS OF A WEB-BASED TAILORED INTERVENTION TO REDUCE ALCOHOL CONSUMPTION IN ADULTS: RANDOMIZED CONTROLLED TRIAL

This chapter has been published as:

Schulz DN, Candel MJJM, Kremers SPJ, Reinwand DA, Jander A, de Vries H. Effects of a web-based tailored intervention to reduce alcohol consumption in adults: randomized controlled trial. Journal of Medical Internet Research 2013; 15:e206. 


\section{ABSTRACT}

Background: Web-based tailored interventions provide users with information that is adapted to their individual characteristics and needs. Randomized controlled trials assessing the effects of tailored alcohol self-help programmes among adults are scarce. Furthermore, it is a challenge to develop programmes that can hold respondents' attention in online interventions.

Objective: To assess whether a three-session, web-based tailored intervention is effective in reducing alcohol intake in high-risk adult drinkers and to compare two computer-tailoring feedback strategies (alternating versus summative) on behavioural change, dropout, and appreciation of the programme.

Methods: A single-blind randomized controlled trial was conducted with an experimental group and a control group ( $N=448$ ) in Germany in 2010-2011. Follow-up took place after six months. Drinking behaviour, health status, motivational determinants, and demographics were assessed among participants recruited via an online access panel. The experimental group was divided into two subgroups. In the alternating condition ( $n=132$ ), the tailored feedback was split into a series of messages discussing individual topics offered while the respondent was filling out the programme. Participants in the summative condition $(n=181)$ received all advice at once after having answered all questions. The actual texts were identical for both conditions. The control group $(n=135)$ only filled in three questionnaires. To identify intervention effects, logistic and linear regression analyses were conducted among complete cases $(n=197)$ and after using multiple imputation.

Results: Among the complete cases (response rate: 197/448, 44.0\%) who did not comply with the German national guideline for low-risk drinking at baseline, $21.1 \%$ of respondents in the experimental group complied after six months compared with $5.8 \%$ in the control group (effect size $=0.42$; OR 2.65; $95 \% \mathrm{Cl} 1.14-6.16 ; p=.02$ ). The experimental group decreased by 3.9 drinks per week compared to 0.4 drinks per week in the control group, but this did not reach statistical significance (effect size $=$ $0.26 ; \beta=-0.12 ; 95 \% \mathrm{Cl}-7.96-0.03 ; \mathrm{p}=.05)$. Intention-to-treat analyses also indicated no statistically significant effect. Separate analyses of the two experimental subgroups showed no differences in intervention effects. The dropout rate during the first visit to the intervention website was significantly lower in the alternating condition than in the summative condition (OR $0.23 ; 95 \% \mathrm{Cl} 0.08-0.60$; $\mathrm{p}=.003)$. Programme appreciation was comparable for the two experimental groups.

Conclusion: Complete case analyses revealed that web-based tailored feedback can be an effective way to reduce alcohol intake among adults. However, this effect was not confirmed when applying multiple imputations. There was no indication that one of the tailoring strategies was more effective in lowering alcohol intake. Nevertheless, the lower attrition rates we found during the first visit suggest that the version of the intervention with alternating questions and advice may be preferred.

Keywords: alcohol intake; adults; eHealth; computer-tailoring; web-based intervention; tailoring methods; effectiveness 


\section{INTRODUCTION}

Although the consumption of alcohol is associated with numerous negative consequences, such as cardiovascular disease, cancer, cirrhosis, neuropsychiatric disorders, traffic accidents, and reduced work productivity (Cherpitel et al., 2006; Rehm et al., 2009; World Health Organization, 2006), high alcohol consumption is highly prevalent among adults worldwide (Rehm et al., 2001; Stock et al., 2009; Van Oers et al., 1999). Many people with unhealthy drinking patterns are not aware of their alcohol intake or the problems associated with this behaviour (Kuntsche et al., 2004; Murgraff et al., 1999); others are aware, but do not seek care, help, or support (Burton \& Williamson, 1995; Cunningham \& Breslin, 2004; Poulin et al., 1997) possibly out of fear, shame, or lack of time. The high prevalence of unhealthy drinkers and the low number of them who seek help underline the need for easily accessible and low-threshold interventions to encourage people to reduce their alcohol intake.

Web-based tailored interventions in which information is adapted to the user's individual characteristics and needs to give them personally appropriate advice (De Vries \& Brug, 1999; Krebs et al., 2010) have proved an effective tool to improve health-related behaviours. Various studies have reported favourable effects on lifestyle behaviours, such as increasing physical activity (Spittaels et al., 2007b; Van Stralen et al., 2011), increasing fruit and vegetable consumption and lowering saturated fat intake (Neville et al., 2009a), and giving up smoking (Dijkstra et al., 1998a). The main advantages of intervention programmes providing tailored advice compared to non-tailored materials are that they contain less unnecessary information and more attractive and relevant information (Kreuter et al., 2000b; Ryan \& Lauver, 2002), they are cost-effective (Smit et al., 2011), the tailored messages are more likely to be read, saved, printed out, remembered, and discussed with others (Campbell et al., 1994; De Vries \& Brug, 1999; Smeets, 2006; Spittaels et al., 2007a), and tailored information is more effective for behaviour change than generic messages (Krebs et al., 2010; Noar et al., 2007; Riper et al., 2008).

To date, several studies of web-based tailored alcohol interventions have been published, but randomized controlled trials among the general adult population using tailored self-help programmes have been scarce (Boon et al., 2011; Cunningham et al., 2009; Cunningham et al., 2010; Cunningham, 2012; Hester et al., 2009; Matano et al., 2007; Riper et al., 2008; Wallace et al., 2011;). Most previous studies were conducted among young people, especially among university or student populations (Bendtsen et al., 2012; Bewick et al., 2008; Bewick et al., 2010; Haug et al., 2012; White et al., 2010). These samples are not representative of the general population and may, for example, differ in motivation to change, reading level, computer and Internet access, and computer literacy (White et al., 2010). Earlier studies reported that single-session, individually personalized feedback without therapeutic guidance can be an effective and cost-effective method to reduce alcohol consumption (Riper et al., 2009). A recently published study of adult men using a single-session intervention in which respondents had to go to a laboratory to participate in an online 10-minute intervention reported only on short-term effects one month after the intervention (Boon et al., 2011). 
Little research has been done to assess what elements work well in tailored interventions. The five criteria of Health Behaviour Change treatment on the Internet (HBC-I) - advise, assist, assess, provide anticipatory guidance, and arrange follow-up - form essential, but not sufficient, elements that determine whether a programme offers potential for behaviour change (Evers et al., 2005). Other feasible elements appear to be the use of tailoring strategies, such as normative, positive, and ipsative feedback, personal tone, and empathy (Dijkstra \& De Vries, 1999). Factors explaining the differences in effectiveness of programmes include the number of contact/exposure moments, the use of theory, the layout, the communication channel, the length of the questionnaires, the amount of information given, and the depth of tailoring (Dijkstra, 2005; Krebs et al., 2010).

Although Internet-based programmes have the potential to reach large numbers of people, various studies have pointed out that the actual use may be limited and that high rates of attrition are common (Brouwer et al., 2009; Brouwer et al., 2010; Glasgow et al., 2007; Leslie et al., 2005; Schulz et al., 2012b; Spittaels et al., 2007b). To prevent early dropout and, thus, increase the effectiveness of a programme, two different strategies could be used to hold respondents' attention in online interventions. In the first strategy, questions and advice are given alternately, so that the respondents are rewarded while they are still filling in the questionnaires and are thereby motivated to continue. Such alternation might also enhance the attractiveness of the programme. In the second strategy, advice is given in a more traditional way at the end of the session (i.e. after the last question has been completed). This method may lead to postponement of dropout - provided that the questionnaires are not too long - because respondents have to wait until the end of the questionnaire before receiving tailored feedback. Yet, this method may also increase the risk that the participant becomes overwhelmed by the amount of information he or she receives all at once (Schulz et al., 2011).

The objective of our study was twofold. First, we explored the overall effectiveness of a three-session, web-based, tailored alcohol intervention for unhealthy drinkers in the general adult population. Second, we compared the dropout rate, effectiveness, and user satisfaction of two kinds of feedback strategies (alternating versus summative).

\section{METHODS}

\section{Participants, procedure and study design}

We conducted a randomized controlled trial (ISRCTN91623132) involving an experimental group and a waiting list control group, with a follow-up measurement after six months. The intervention, focusing on unhealthy drinkers in the general population, was conducted online in Germany in June 2010 to January 2011. Adult participants were recruited via an online access panel (i.e. a register of a sample who expressed willingness to participate in online surveys and research studies) called respondi AG (place of business: Cologne, Germany). The sample received an e-mail containing a link to either the intervention website (experimental group) or a web-based alcohol questionnaire (control group). Randomization was carried out by a computer system. Two reminder messages in the form of e-mails 
were sent to individuals of the sample who did not respond to the first invitation. Incentives, in the form of bonus points that respondents could exchange for cash, a gift voucher, or a charitable donation were given to respondents who filled in the questionnaires completely. Informed consent was given during the registration process as a panel member in which the members gave permission to use their data for scientific research.

\section{Inclusion criteria}

The following inclusion criteria were established for this study: being a panel member of respondi; having computer/Internet literacy; having sufficient command of German; being 18 years or older; and having an unhealthy drinking pattern, which was defined as (1) not complying with the guideline recommending no more than one glass (women) or two glasses (men) of alcohol per day, (2) drinking on more than five days per week, (3) having a score higher than seven on the Alcohol Use Disorders Identification Test (AUDIT) (Saunders et al., 1993), or (4) currently trying to become pregnant, drinking alcohol while pregnant or breastfeeding (in relation to pregnancy), or trying to get one's partner pregnant (for men).

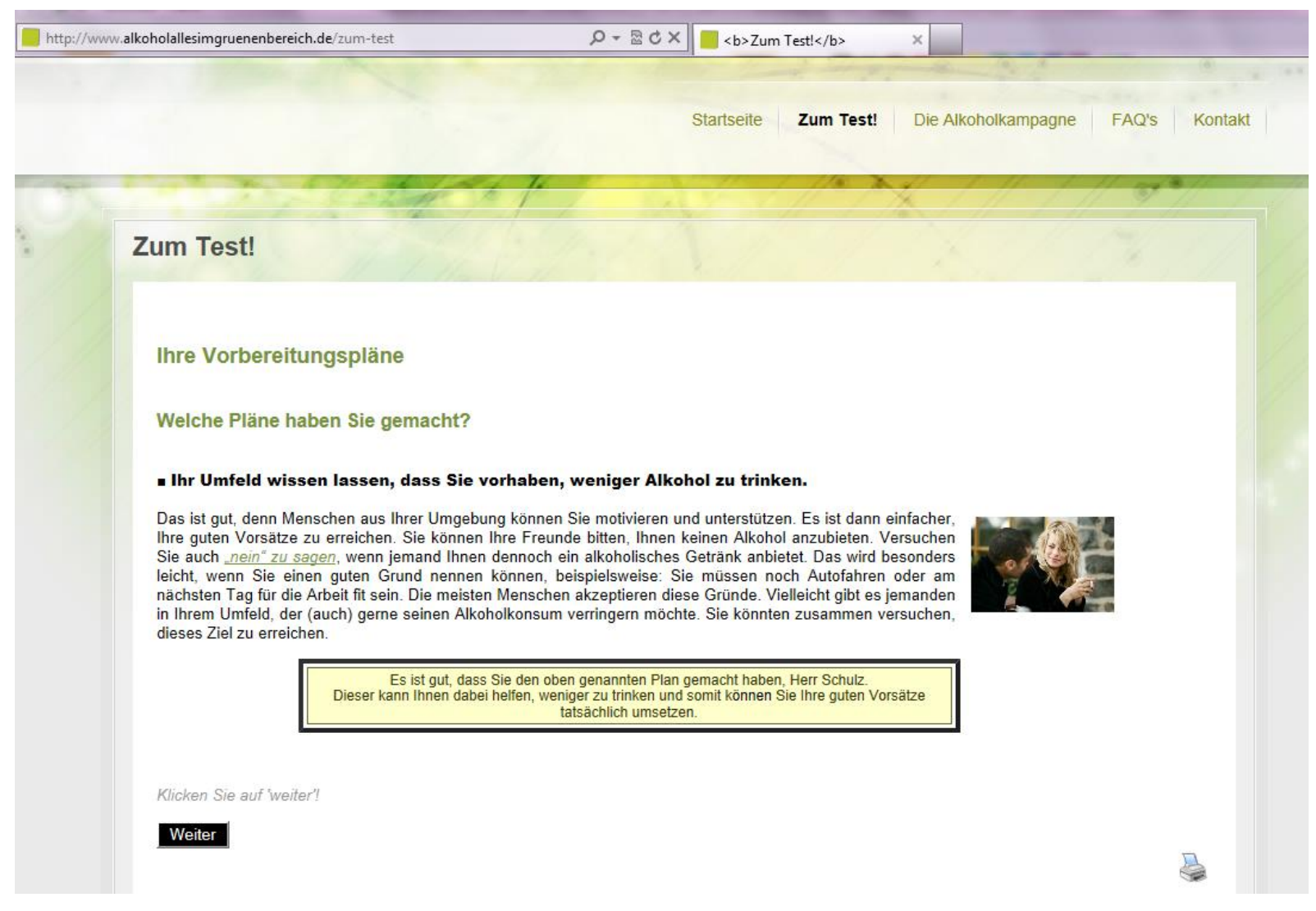

Figure 6.1 Screenshot of the intervention website, showing personal advice regarding preparatory plans 


\section{Intervention}

The intervention programme, called Alcohol - Everything within the Limits?! (German: "Alkohol - Alles im grünen Bereich?!," see Figure 6.1), is a web-based, three-session, tailored programme targeting adult problem drinkers. The main aim of the intervention was to stimulate participants to lower their alcohol intake. The theoretical framework for the development of the intervention was the I-Change model (De Vries et al., 2003). This psychosocial model was chosen because it combines different models and integrates these in premotivational, motivational, and postmotivational phases, which is optimal for use in computer-tailoring to support the process of behavioural change. The I-Change model builds on other psychosocial models, such as the Theory of Planned Behaviour (Ajzen, 1991), Social Cognitive Theory (Bandura, 1986), the Health Belief Model (Janz \& Becker, 1984), and the Transtheoretical Model (Prochaska \& DiClemente, 1983).

The personalized advice, which was presented immediately on the respondent's computer screen, consisted of five parts, each focusing on a different psychosocial construct of the model (i.e. knowledge, awareness, attitude, social influence, self-efficacy, and action planning). The first part of the programme served as a starting point of the drinking behaviour change process (premotivational phase) by addressing the concepts of knowledge and awareness: it gave information about the German alcohol guidelines, specifically, not drinking more than one (women) or two (men) standard drinks (i.e. drinks containing 10 grams of alcohol) per day and having at least two alcohol-free days a week, and assessed whether respondents were meeting this guideline by using comparative/normative feedback. In addition, respondents' scores were depicted graphically using a traffic light symbol (indicating whether they met, almost met, or did not meet the guidelines). To increase the respondent's level of knowledge, the relation between alcohol and various diseases was explained, and information tailored to the respondent's health status was given about alcohol and pregnancy, and about the possible influence of participants' drinking behaviour on their children (if applicable). The second part of the programme offered personalized feedback concerning the perceived pros and cons of alcohol drinking as perceived by the respondent, with the goal of creating a positive attitude toward not drinking more than one (women) or two (men) alcoholic drinks per day. The third part explained the importance of social influence in a tailored message by focusing on the respondent's partner, family, friends, and colleagues. In the fourth part, preparatory action plans were defined to prepare the intended behavioural change. The final part focused on self-efficacy and coping plans by identifying difficult situations and suggesting ways to cope with them. Personalized tips were given on how to deal with the perceived difficult situations to overcome potential barriers (postmotivational phase), and the situations and plans were summarized for individual respondents to help them remember these.

During the feedback moment after three months and the follow-up measurement after six months, participants of the experimental group again received personalized advice based on their previous scores for the psychosocial constructs. Additionally, ipsative feedback was given about the respondents' alcohol intake by comparing the drinking score at the current visit with that at the last 
visit or visits. Feedback was given about potential change and all scores were illustrated in a graph to enable the respondent to monitor the total change process at a glance.

\section{Conditions}

All study groups received identical questionnaires. After completing the third measurement, respondents in the waiting list control group were given the link to the intervention website where they could also receive personalized advice. The experimental condition was divided into two subgroups. The intervention website for these two subgroups offered the same feedback messages. At all three feedback moments (at baseline, after three months, and after six months), one experimental subgroup received questions and personal advice alternately (alternating condition) whereas respondents in the other experimental subgroup were given all personal advice at once after having answered all the questions (summative condition). In other words, in the alternating condition, the feedback message was split into a series of messages discussing individual topics offered while the respondent was still completing the web-based session, whereas in the summative condition, the entire set of materials/feedback messages was provided at one time at the end of the web-based session. The actual texts were identical for both conditions. Both subgroups also received a full overview of their advice (equivalent to approximately seven to ten A4 pages of text, including pictures and graphics) at the end of a session/measurement, which they could print or save onto their computer. We gave personalized feedback again after six months to stimulate participation and to enable us to reassess user satisfaction with the programme.

\section{Questionnaires}

\section{Drinking behaviour}

Weekly alcohol intake was measured by the widely used Dutch five-item Quantity-FrequencyVariability (QFV) questionnaire (Lemmens et al., 1992). The Alcohol Use Disorder Identification Test (AUDIT) was used to identify problem drinking (Saunders et al., 1993). Habitual drinking behaviour was assessed by the 12-item Self-Report Habit Index (SRHI) questionnaire (Verplanken \& Orbell, 2003).

\section{Psychosocial determinants}

Knowledge regarding the national alcohol guideline was assessed by one question: "What do you think is the standard acceptable alcohol amount per day and per week?" with 14 answering options, such as "Two glasses every day is allowed." A knowledge test was included in the final measurement for all three conditions, consisting of nine questions, such as "How much alcohol is recommended (i.e. permitted without having to worry about unfavourable consequences) during pregnancy?" or "How much alcohol does a standard drink contain?" 
Attitude was assessed by six pros and six cons of alcohol intake, such as "Drinking alcohol...allows me to relax" and "...is bad for my health" ( $1=$ totally disagree; $5=$ totally agree; pros alpha $=.83$, cons alpha $=.71)$.

Social influence was assessed by dividing this concept into norm, modelling, and support. Norm was assessed using the following item "According to people in my immediate environment, I should definitely drink no more than one glass (women) or two glasses (men) of alcohol a day $(=1)$ " to "I should definitely drink more than one glass (women) or two glasses (men) of alcohol a day (=5)." Modelling was assessed by asking "How many people in your immediate environment drink no more than one glass (women) or two glasses (men) of alcohol a day?" ( 1 = nobody; 5 = everybody). Support was assessed by including the statement "People in my direct environment support me in my efforts to drink no more than one glass (women) or two glasses (men) of alcohol a day" ( $1=$ no, they don't support me at all; 4 = yes, they support me very much).

Self-efficacy was assessed by six items regarding difficult social, emotional, and routine situations, such as "I'm able to meet the alcohol guideline... when I'm at a party," "...when I feel stressed or nervous," and "...during a meal" ( $1=$ no, definitely not; $5=$ yes, definitely; alpha $=.81)$.

Preparatory plans were assessed by four items, such as "I'm planning to take less money with me when I go out, so I can't buy a lot of alcoholic drinks" ( 1 = no, definitely not; 5 = yes, definitely; alpha = .77).

Coping plans were assessed by six items regarding the various risk situations, such as "I've made a plan to drink no more than one glass (women) or two glasses (men) of alcohol when I feel stressed or nervous" ( 1 = totally disagree; 5 = totally agree; alpha $=.96)$.

Motivational stage of drinking in accordance with the alcohol guideline was assessed by applying the Transtheoretical Model of Behaviour Change (Prochaska \& DiClemente, 1983). We used one item: "Do you intend to drink on no more than five days per week and no more than one glass (women) or two glasses (men) of alcohol a day?" ( $1=$ no, I don't intend to do so; $2=1$ never thought about it; $3=1$ thought about it, but I don't know yet; 4 = yes, but not within the next 5 years; yes, $5=$ within $1-5$ years; 6 = yes, within 6-12 months, $7=$ yes, within 3-6 months; $8=$ yes, within $1-3$ months; $9=$ yes, within a month; 10 = yes, and I'm already doing so).

\section{Health status}

Six items were used to assess if respondents suffered from diabetes mellitus, angina pectoris, cancer, or high blood pressure or had suffered a stroke or cardiac infarction. Symptoms of depression were assessed by means of the 10-item Centre for Epidemiologic Studies Depression Scale (CES-D10) (Andresen et al., 1994). 


\section{Demographic information}

The following demographic variables were assessed: age, gender ( $1=$ male; 2 female), educational level $(1=$ low/no education or primary education; $2=$ medium $/$ secondary education; $3=$ high/tertiary education), income (euros per month), employment situation ( 1 = paid employment; 2 = no paid employment), marital status, pregnancy/breastfeeding status ( $1=$ pregnant/breastfeeding and drinking; $2=n / a)$, number of children living at home, and native country ( $1=$ Germany; 2 = other country).

\section{Appreciation of the programme}

Both experimental subgroups were invited to fill in an evaluation questionnaire to assess the levels of personalization and their appreciation of the intervention. Seven questions were included, such as "The personal advice I received was interesting" ( $1=$ no, absolutely not; $5=$ yes, absolutely).

\section{Primary objective}

The primary objective was to compare the experimental group (i.e. the subgroups who received the computer-tailored feedback strategies) with the control group regarding (1) complying with the alcohol guideline (healthy drinking; yes/no) after six months, and (2) mean weekly alcohol consumption (in number of standard drinks) at six months after baseline.

\section{Secondary objective}

The second objective was to compare the two computer-tailored feedback strategies (alternating versus summative) in terms of dropout rates, effects on drinking behaviour, and appreciation of the programme.

\section{Power analyses}

We estimated the required sample size for both the logistic regression analysis and the linear regression analysis based on the intervention effects of a comparable study by Riper et al. (2008). For the logistic regression analysis, a power analysis calculation indicated that a total sample of 180 respondents was needed (after possible attrition) to test for the intervention effect. We calculated the sample size for two experimental groups and a control group based on a .05 level of significance, a statistical power of $80 \%$, and a two-sided test. We expected that the compliance with the guideline would be $20 \%$ in the intervention groups and $5 \%$ in the control group. For the linear regression analysis, a power analysis calculation indicated that a total sample of 254 respondents was needed 
(after possible attrition) to test for the intervention effect. Again, we calculated the sample size for two experimental groups and a control group, based on a .05 level of significance, a statistical power of $80 \%$, a two-sided test, an effect size (ES) of 0.30 (when contrasting the two intervention groups with the control group), and a correlation of 0.60 between premeasurement and postmeasurement of the outcome variable.

\section{Statistical analyses}

The data were analysed using SPSS software, version 19 (IBM Corp, Armonk, NY, USA). To check whether the randomization had been successful in terms of demographics and drinking behaviour, linear regression analyses were used for continuous variables and chi-square tests for discrete variables. Descriptive statistics were used for the characteristics of the study sample and the dropout rate within the groups. Logistic regression analyses were performed to determine differences in dropout rates between the study conditions.

The two experimental groups together were compared to the control group for drinking behaviour. First, effect sizes were calculated based on means and odds ratios (Cohen's d). Effect sizes below 0.30 were considered small, whereas those between 0.30 and 0.80 were considered medium, and those greater than 0.80 were regarded as large (Cohen, 1988). Second, differences in effect between the groups were explored by means of logistic as well as linear regression analyses. The following baseline variables were entered as independent variables in both types of regression analyses using the backward method: condition, gender, age, educational level, employment status, income, country of birth, marital status, having children, pregnancy, disease, CES-D10, number of alcoholic drinks, AUDIT, SRHI, pros, cons, social support, social modelling, social norm, self-efficacy, coping plans, and intention. Preparatory plans were not included in the analyses because not every participant was presented with these items. The dependent variables were (1) meeting the guideline $(0=$ no; $1=$ yes), and (2) the weekly number of alcoholic drinks after six months.

Linear regression analyses were used to determine differences in programme evaluation between the two experimental subgroups. The dependent variables were the separate items regarding appreciation of the programme. Those demographic variables that differed between the study groups were included in the analyses as covariates.

Tests were performed at alpha $=.05$ for the intervention factor and alpha $=.10$ for covariates (Rosnow $\&$ Rosenthal, 1989). Analyses were done on data for complete cases only as well as intention-to-treat (ITT) analyses, in which multiple imputation (Schafer, 1999) was used to fill in missing values. Missing values were filled using demographics, health status, psychosocial determinants, baseline drinking behaviour, drinking behaviour after three and six months, and study condition as predictors. The number of imputations was set at 55. This was done according to the recommendation to create as many imputed datasets as the percentage of cases with missing data (Bodner, 2008). In addition, we also conducted a sensitivity analysis in which the last observation carried forward (LOCF) method was used to fill in missing values. 


\section{RESULTS}

\section{Participation and attrition}

Figure 6.2 presents a flowchart for the study participants. A total of 1,149 participants logged on to the programme; 614 did not meet the inclusion criteria and 87 respondents provided incomplete or missing data, resulting in a total sample size of 448 respondents. At the three-month feedback moment, loss to follow-up was 31.3\% (140/448). The dropout rate differed significantly among the three conditions $(p=.001)$ : dropout was significantly lower in the control condition compared to the alternating condition (OR $0.40 ; 95 \% \mathrm{Cl} 0.22-0.73 ; p=.003$ ) and compared to the summative condition (OR 0.35; 95\% Cl 0.19-0.63; $\mathrm{p}$ <.001). Moreover, dropout was lower among men (OR 1.48; $95 \% \mathrm{Cl} 0.95-2.32 ; \mathrm{p}=.08$ ) and among respondents with a high educational level compared to those with a low educational level (OR 0.60; $95 \% \mathrm{Cl} 0.36-1.00 ; \mathrm{p}=.05$ ) although this did not reach statistical significance. At six months, loss to follow-up was 36.8\% (165/448) and the dropout rate was distributed equally among the three conditions $(p=.74)$; however, there was a significant difference in dropout among respondents with different levels of income $(p=.03)$; the dropout rate was lower in respondents with the highest income compared to those with the lowest income (OR 0.36; $95 \% \mathrm{Cl}$ $0.15-0.83 ; p=.02)$.

\section{Differences in completion rate between the experimental subgroups}

During the first session, the dropout rate of the intervention was significantly lower in the alternating condition compared to the summative condition. In the alternating condition, 96.2\% (127/132) completed the programme whereas in the summative condition, 85.1\% (154/181) did so (OR 0.23; $95 \% \mathrm{Cl} 0.08-0.60 ; \mathrm{p}=.003)$. However, differences regarding attrition were no longer significant after three and six months. After three months, 62.1\% (82/132) of those in the alternating condition and $55.2 \%(100 / 181)$ of those in the summative condition returned to the website and filled in the programme completely (OR 0.94; 95\% Cl $0.57-1.54 ; p=.80)$. At six-month follow-up, 56.8\% (75/132) of those in the alternating condition and 58.6\% (106/181) of those in the summative condition returned to the website and filled in the programme completely (OR 1.24; 95\% Cl $0.77-2.01 ; \mathrm{p}=.38$ ).

\section{Sample characteristics}

Slightly more men than women were included in the study and the mean age of the respondents was approximately 42 years. The average weekly alcohol intake was almost 13 glasses. The baseline demographic characteristics of the study sample are shown in Table 6.1. Significant differences at the $p<.10$ level were found for the baseline characteristic of income $\left(\chi^{2}{ }_{6}=14.70 ; p=.02\right)$ and habitual drinking ( $\beta=0.10 ; 95 \% \mathrm{Cl} 0.00-0.18 ; p=.04)$. 
Table 6.1 Demographics, health status, and drinking behaviour of the study sample at baseline

\begin{tabular}{|c|c|c|c|c|}
\hline Variable & $\begin{array}{r}\text { Total } \\
\mathrm{N}=448\end{array}$ & $\begin{array}{r}\text { Alternating } \\
\text { condition } \\
n=132\end{array}$ & $\begin{array}{r}\text { Summative } \\
\text { condition } \\
n=181\end{array}$ & $\begin{array}{r}\text { Control } \\
\text { condition } \\
n=135\end{array}$ \\
\hline Age (18-69 years) & $41.72(S D=15.74)$ & $42.23(S D=15.06)^{a}$ & $41.41(S D=16.16)$ & $41.62(S D=15.92)$ \\
\hline \multicolumn{5}{|l|}{ Gender } \\
\hline Male & $253(56.5 \%)$ & $69(52.3 \%)$ & $104(57.5 \%)$ & $80(59.3 \%)$ \\
\hline Female & $195(43.5 \%)$ & $63(47.7 \%)$ & 77 (42.5\%) & $55(40.7 \%)$ \\
\hline \multicolumn{5}{|l|}{ Education } \\
\hline Low & $177(42.0 \%)$ & $61(47.3 \%)$ & 61 (38.9\%) & 55 (40.7\%) \\
\hline Medium & $101(24.0 \%)$ & $25(19.4 \%)$ & $40(25.5 \%)$ & $36(26.7 \%)$ \\
\hline High & $143(34.0 \%)$ & $43(33.3 \%)$ & $56(35.7 \%)$ & $44(32.6 \%)$ \\
\hline \multicolumn{5}{|l|}{ Income per month } \\
\hline$<€ 1,000$ & $61(13.6 \%)$ & $11(8.3 \%)$ & $24(13.3 \%)$ & $26(19.3 \%)$ \\
\hline$€ 1,001-€ 2,000$ & $106(23.7 \%)$ & $41(31.1 \%)$ & $30(16.6 \%)$ & $35(25.9 \%)$ \\
\hline$€ 2,001-€ 4,000$ & $135(30.1 \%)$ & $34(25.8 \%)$ & 55 (30.4\%) & 46 (34.1\%) \\
\hline$>€ 4,000$ & $43(9.6 \%)$ & $19(14.4 \%)$ & $12(6.6 \%)$ & $12(8.9 \%)$ \\
\hline Not reported & $103(23.0 \%)$ & $27(20.5 \%)$ & $60(33.1 \%)$ & 16 (11.9\%) \\
\hline \multicolumn{5}{|l|}{ Employment situation } \\
\hline Job (paid employment) & $269(65.3 \%)$ & $89(71.8 \%)$ & 97 (63.4\%) & 83 (61.5\%) \\
\hline No job & $143(34.7 \%)$ & $35(28.2 \%)$ & $56(36.6 \%)$ & $52(38.5 \%)$ \\
\hline \multicolumn{5}{|l|}{ Marital status } \\
\hline Married & $170(40.4 \%)$ & $55(42.6 \%)$ & $62(39.5 \%)$ & $53(39.3 \%)$ \\
\hline Living together & 67 (14.9\%) & $26(20.2 \%)$ & $28(17.8 \%)$ & $13(9.6 \%)$ \\
\hline In relationship, not living together & $51(12.1 \%)$ & $12(9.3 \%)$ & $22(14.0 \%)$ & $17(12.6 \%)$ \\
\hline Single/unmarried & $90(21.4 \%)$ & $22(17.1 \%)$ & $35(22.3 \%)$ & $33(24.4 \%)$ \\
\hline Divorced & $31(7.4 \%)$ & $9(7.0 \%)$ & $6(3.8 \%)$ & 16 (11.9\%) \\
\hline Widowed & $12(2.9 \%)$ & $5(3.9 \%)$ & $4(2.5 \%)$ & $3(2.2 \%)$ \\
\hline \multicolumn{5}{|l|}{ Children } \\
\hline No & $226(50.4 \%)$ & $58(43.9 \%)$ & $100(55.2 \%)$ & $68(50.4 \%)$ \\
\hline Yes, but no longer living at home & $93(20.8 \%)$ & $26(19.7 \%)$ & $33(18.2 \%)$ & $34(25.2 \%)$ \\
\hline Yes, living at home $>18$ years & $34(7.6 \%)$ & $14(10.6 \%)$ & $11(6.1 \%)$ & $9(6.7 \%)$ \\
\hline Yes, living at home $<18$ years & $95(21.2 \%)$ & $34(25.8 \%)$ & $37(20.4 \%)$ & $24(17.8 \%)$ \\
\hline \multicolumn{5}{|l|}{ Native country } \\
\hline Germany & 409 (97.1\%) & $126(97.7 \%)$ & $152(96.8 \%)$ & $131(97.0 \%)$ \\
\hline Other & $12(2.9 \%)$ & $3(2.3 \%)$ & $5(3.2 \%)$ & $4(3.0 \%)$ \\
\hline \multicolumn{5}{|l|}{ Symptoms of depression } \\
\hline CES-D10 $b$ & $8.20(S D=5.05)$ & $8.08(S D=5.46)$ & $8.38(S D=5.05)$ & $8.11(S D=4.68)$ \\
\hline Score of $\geq 11$ & $120(28.8 \%)$ & $39(30.7 \%)$ & $44(28.6 \%)$ & $37(27.4 \%)$ \\
\hline \multicolumn{5}{|l|}{ Diseases } \\
\hline Diabetes mellitus & $21(4.7 \%)$ & $7(5.2 \%)$ & $9(5.0 \%)$ & $5(3.7 \%)$ \\
\hline Stroke & $8(1.8 \%)$ & $1(0.7 \%)$ & $3(1.7 \%)$ & $4(3.0 \%)$ \\
\hline Cardiac infarction & $7(1.6 \%)$ & $1(0.7 \%)$ & $3(1.7 \%)$ & $3(2.2 \%)$ \\
\hline Angina pectoris & $9(2.0 \%)$ & $2(1.5 \%)$ & $4(2.2 \%)$ & $3(2.2 \%)$ \\
\hline Cancer & $6(1.3 \%)$ & $0(0.0 \%)$ & $4(2.2 \%)$ & $2(1.5 \%)$ \\
\hline High blood pressure & $95(21.1 \%)$ & $26(19.3 \%)$ & $41(22.7 \%)$ & $28(20.7 \%)$ \\
\hline One or more diseases & $128(28.6 \%)$ & $35(26.5 \%)$ & $55(30.4 \%)$ & $38(28.1 \%)$ \\
\hline \multicolumn{5}{|l|}{ Alcohol } \\
\hline Non-adherence to guideline & $221(51.4 \%)$ & $63(47.7 \%)$ & 85 (49.7\%) & $73(54.9 \%)$ \\
\hline Weekly alc. intake (standard units) c & $12.94(S D=11.24)$ & $12.53(\mathrm{SD}=10.99)$ & $11.86(\mathrm{SD}=9.70)$ & $14.73(S D=13.05)$ \\
\hline Pregnant/breastfeeding \& drinking & $31(6.9 \%)$ & $8(6.1 \%)$ & $14(7.7 \%)$ & $9(6.7 \%)$ \\
\hline AUDIT (score $\geq 8$ ) & $351(80.0 \%)$ & 102 (77.3\%) & 141 (79.2\%) & $108(81.2 \%)$ \\
\hline Habit (SRHI-12)d & $2.11(S D=0.82)$ & $1.98(S D=0.79)$ & $2.15(\mathrm{SD}=0.79)$ & $2.19(S D=0.86)$ \\
\hline
\end{tabular}




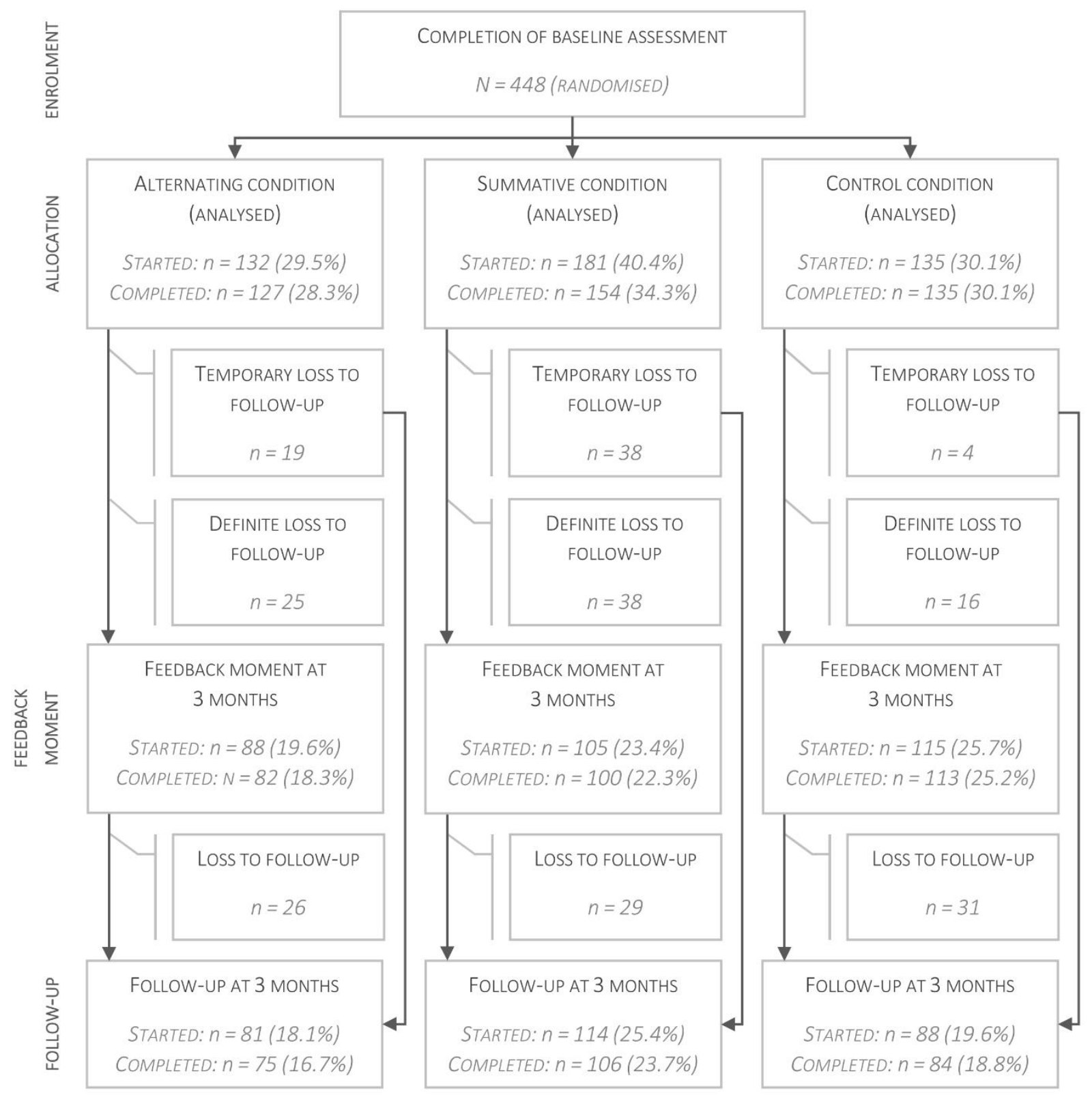

Figure 6.2 Flowchart of the study sample

\section{Intervention effects}

The number of respondents who complied with the alcohol guideline rose after six months (Figure 6.3). The percentage of respondents complying with the guideline increased by $21.1 \%$ in the experimental group and by $5.8 \%$ in the control group. The number of alcoholic drinks per week among the study population also decreased during the intervention period (Figure 6.3). The experimental group reduced their mean weekly alcohol intake by 3.9 drinks (SD $=9.96)$ compared to 0.4 drinks $(S D=19.54)$ in the control group. 
- -xperimental group

$E S=0.42$

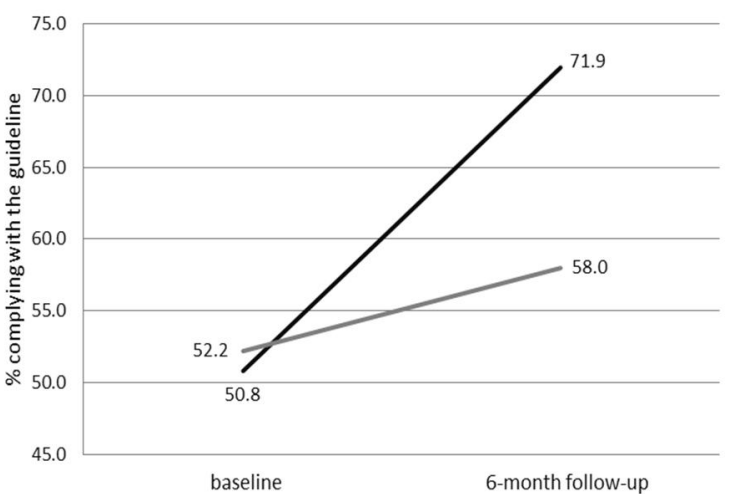

Control group

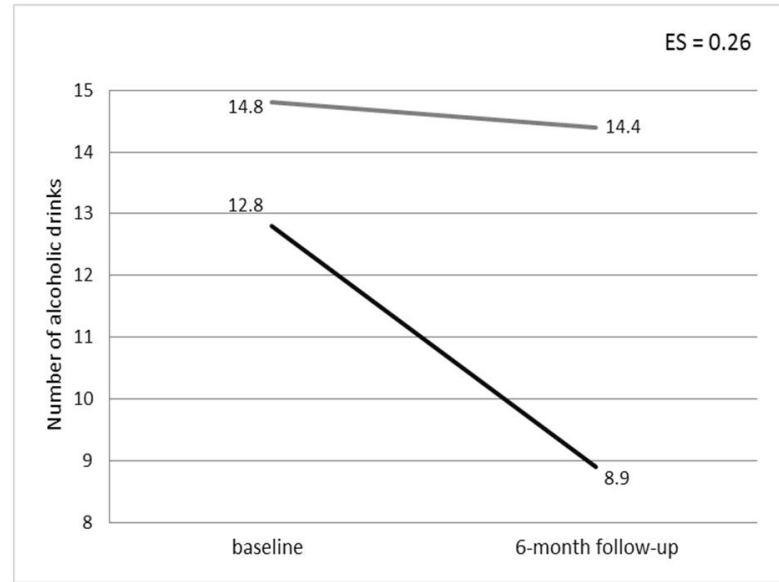

Figure 6.3 Differences and effect sizes (ES) regarding compliance with the alcohol guideline among complete cases $(n=197)$ and number of alcoholic drinks per week between the experimental group and the control group at baseline and after six months

As shown in Table 6.2, the results of the logistic regression analysis showed that the intervention was effective in achieving a low-risk drinking status according to the guideline among complete cases (OR 2.65; $p=.02$ ). However, different results were found when using ITT analyses. After applying multiple imputations, no intervention effect was found among the study sample (OR $1.11 ; p=.72$ ). Results of the sensitivity analysis using LOCF can be found in Appendix 1.

Table 6.2 Results of the logistic regression analysis (backward method) with guideline status ( 0 = not complying; 1 = complying) after six months as dependent variable among complete cases (CC, $n=197)$ and after applying multiple imputations ( $\mathrm{MI}, \mathrm{n}=448$ )

\begin{tabular}{|c|c|c|c|c|c|c|}
\hline \multirow[t]{2}{*}{ Variable $^{a}$} & \multicolumn{3}{|c|}{ Guideline status (CC) } & \multicolumn{3}{|c|}{ Guideline status (MI) } \\
\hline & OR & $p$ & $95 \% \mathrm{Cl}$ & OR & $p$ & $95 \% \mathrm{Cl}$ \\
\hline Condition & 2.65 & .02 & $1.14-6.16$ & 1.11 & .72 & $0.63-1.98$ \\
\hline Guideline status & - & - & - & 2.91 & $<.001$ & $1.63-5.18$ \\
\hline Weekly alcohol intake & 0.88 & $<.001$ & $0.84-0.93$ & 0.96 & .04 & $0.93-1.00$ \\
\hline Habit & 0.23 & $<.001$ & $0.12-0.42$ & 0.46 & $<.001$ & $0.31-0.70$ \\
\hline AUDIT & 0.40 & .07 & $0.15-1.09$ & - & - & - \\
\hline Age & 0.96 & .007 & $0.94-0.99$ & - & - & - \\
\hline Self-efficacy & 0.47 & .03 & $0.24-0.94$ & 0.62 & .045 & $0.39-0.99$ \\
\hline Intention & 0.88 & .03 & $0.78-0.98$ & - & - & - \\
\hline $\mathrm{R}^{2}$ & 0.52 & & & 0.32 & & \\
\hline
\end{tabular}

Note: ${ }^{a}$ Assessed at baseline. 
The linear regression analysis among complete cases (see Table 6.3) found an effect for the intervention in lowering the weekly number of alcoholic beverages in the experimental group, but this did not reach statistical significance $(\beta=-0.12 ; 95 \% \mathrm{Cl}-7.96-0.03 ; p=.05)$. After applying multiple imputations, no intervention effect was found $(B=-1.15 ; 95 \% \mathrm{Cl}-4.02-1.72 ; \mathrm{p}=.43)$.

Table 6.3 Results of the linear regression analysis (backward method) with the number of alcoholic drinks after six months as dependent variable among complete cases (CC, $n=197)$ and after applying multiple imputations $(\mathrm{MI}, \mathrm{n}=448)$

\begin{tabular}{|c|c|c|c|c|c|c|}
\hline \multirow[t]{2}{*}{ Variable $^{a}$} & \multicolumn{3}{|c|}{ Number of drinks (CC) } & \multicolumn{3}{|c|}{ Number of drinks (MI) } \\
\hline & $\beta$ & $p$ & $95 \% \mathrm{Cl}$ & $\mathrm{B}$ & $p$ & $95 \% \mathrm{Cl}$ \\
\hline Condition & -0.12 & .05 & $-7.96-0.03$ & -1.15 & .43 & $-4.02-1.72$ \\
\hline Weekly alcohol intake & 0.49 & $<.001$ & $0.52-0.86$ & 0.61 & $<.001$ & $0.47,-0.75$ \\
\hline Habit & 0.18 & .01 & $0.77-60.36$ & 2.65 & .01 & $0.67-4.64$ \\
\hline Native country & 0.10 & .09 & $-1.21-18.11$ & - & - & - \\
\hline Social norm & - & - & - & -1.20 & .049 & $-2.39--0.01$ \\
\hline Self-efficacy & 0.14 & .049 & $0.01-6.17$ & 2.08 & .06 & $-0.12-4.29$ \\
\hline $\mathrm{R}^{2}$ & 0.33 & & & 0.29 & & \\
\hline
\end{tabular}

Note: ${ }^{a}$ Assessed at baseline.

\section{Differences between the two experimental subgroups}

A comparison between the two experimental subgroups $(n=128)$ regarding compliance with the guideline (OR 0.41, 95\% Cl $0.13-1.36, p=.15)$ and weekly alcohol intake $(\beta=-0.03,95 \% \mathrm{Cl}-3.14-$ 2.11, $p=.70$ ) showed no differences in effect. Comparable results were found after multiple imputations of missing values. There were neither differences regarding achievement of low-risk drinking status according to the guideline (OR $0.72,95 \% \mathrm{Cl} 0.38-1.37, \mathrm{p}=.31$ ) nor regarding the weekly number of alcoholic beverages after six months $(B=-0.11,95 \% \mathrm{Cl}-2.89-2.68, p=.94)$ between the two experimental subgroups.

\section{Differences in appreciation of the programme}

In general, the intervention was evaluated positively by both experimental subgroups (Table 6.4). At baseline, respondents of the alternating condition reported that they had read more of the advice compared to respondents of the summative condition; however, this difference did not meet statistical significance $(p=.07)$. After six months, this difference was not apparent. At the six-month follow-up measurement, the advice was perceived as more informative among the summative condition compared to the alternating condition, although this did not meet statistical significance $(p=.08)$. 
Table 6.4 Differences between the two experimental subgroups (alternating condition: $\mathrm{n}=59$; summative condition: $\mathrm{n}=72$ ) regarding the evaluation items about appreciation of the programme

\begin{tabular}{|c|c|c|c|c|c|c|c|c|}
\hline \multirow[t]{2}{*}{ Items } & \multicolumn{4}{|c|}{ Baseline } & \multicolumn{4}{|c|}{ 6-month follow-up } \\
\hline & $\begin{array}{r}\text { Alternating } \\
\text { condition } \\
\text { Mean }\end{array}$ & $\begin{array}{r}\text { Summative } \\
\text { condition } \\
\text { Mean }\end{array}$ & $\beta$ & $p$ & $\begin{array}{r}\text { Alternating } \\
\text { condition } \\
\text { Mean }\end{array}$ & $\begin{array}{r}\text { Summative } \\
\text { condition } \\
\text { Mean }\end{array}$ & $\beta$ & $p$ \\
\hline Evaluation mark ${ }^{a}$ & $\begin{array}{r}11.31 \\
(\mathrm{SD}=3.22)\end{array}$ & $\begin{array}{r}11.51 \\
(\mathrm{SD}=3.29)\end{array}$ & 0.03 & .72 & $\begin{array}{r}12.05 \\
(\mathrm{SD}=2.99)\end{array}$ & $\begin{array}{r}12.08 \\
(\mathrm{SD}=3.24)\end{array}$ & 0.01 & .95 \\
\hline $\begin{array}{l}\text { I have read all } \\
\text { pieces of advice }\end{array}$ & $\begin{array}{r}4.34 \\
(S D=0.78)\end{array}$ & $\begin{array}{r}4.07 \\
(S D=0.89)\end{array}$ & -0.16 & .07 & $\begin{array}{r}3.95 \\
(\mathrm{SD}=0.92)\end{array}$ & $\begin{array}{r}3.92 \\
(\mathrm{SD}=0.88)\end{array}$ & -0.02 & .84 \\
\hline $\begin{array}{l}\text { The advice was } \\
\text { interesting } b\end{array}$ & $\begin{array}{r}4.31 \\
(S D=0.90)\end{array}$ & $\begin{array}{r}4.31 \\
(S D=0.82)\end{array}$ & 0.00 & .99 & $\begin{array}{r}4.32 \\
(\mathrm{SD}=0.88)\end{array}$ & $\begin{array}{r}4.36 \\
(S D=0.74)\end{array}$ & 0.01 & .89 \\
\hline $\begin{array}{l}\text { The advice was } \\
\text { credible }^{b}\end{array}$ & $\begin{array}{r}4.56 \\
(S D=0.68)\end{array}$ & $\begin{array}{r}4.40 \\
(S D=0.80)\end{array}$ & -0.11 & .24 & $\begin{array}{r}4.46 \\
(S D=0.84)\end{array}$ & $\begin{array}{r}4.54 \\
(S D=0.69)\end{array}$ & 0.06 & .53 \\
\hline $\begin{array}{l}\text { The advice was } \\
\text { informative }^{b}\end{array}$ & $\begin{array}{r}4.46 \\
(S D=0.90)\end{array}$ & $\begin{array}{r}4.39 \\
(\mathrm{SD}=0.78)\end{array}$ & -0.04 & .64 & $\begin{array}{r}4.32 \\
(S D=0.92)\end{array}$ & $\begin{array}{r}4.57 \\
(S D=0.71)\end{array}$ & 0.15 & .08 \\
\hline $\begin{array}{l}\text { The advice was } \\
\text { clear }^{b}\end{array}$ & $\begin{array}{r}4.53 \\
(\mathrm{SD}=0.73)\end{array}$ & $\begin{array}{r}4.44 \\
(\mathrm{SD}=0.73)\end{array}$ & -0.06 & .53 & $\begin{array}{r}4.34 \\
(S D=0.86)\end{array}$ & $\begin{array}{r}4.50 \\
(\mathrm{SD}=0.73)\end{array}$ & 0.13 & .14 \\
\hline $\begin{array}{l}\text { The advice helps } \\
\text { me to drink less } \\
\text { alcohol b }\end{array}$ & $\begin{array}{r}3.41 \\
(\mathrm{SD}=1.19)\end{array}$ & $\begin{array}{r}3.47 \\
(S D=1.07)\end{array}$ & 0.03 & .74 & $\begin{array}{r}3.75 \\
(\mathrm{SD}=1.09)\end{array}$ & $\begin{array}{r}3.68 \\
(\mathrm{SD}=1.07)\end{array}$ & 0.01 & .93 \\
\hline
\end{tabular}

Note: ${ }^{a}$ Scores: 0 (very bad)-15 (excellent); ${ }^{b}$ Scores: 1 (no, absolutely not)-5 (yes, absolutely).

\section{DISCUSSION}

\section{Principal findings}

This study used a randomized controlled trial to determine the effectiveness of a web-based tailored alcohol intervention, and to compare the effects of two tailoring strategies in terms of drinking behaviour change, dropout rates, and appreciation. The experimental group and the control group both decreased their alcohol consumption, but the effects for our primary outcomes were greater in the experimental group. Complete case analyses as well as ITT analyses were performed. Inconsistent results were found. First of all, only among complete cases intervention effects were identified in terms of meeting the alcohol guidelines. Second, the experimental group reduced their weekly alcohol intake by a greater amount than the control group, although this effect did not reach statistical significance when performing complete case analyses and multiple imputations.

The results of this study partly confirm that web-based tailored self-help interventions can be an effective tool in decreasing alcohol consumption and encouraging low-risk drinking in adults (Cunningham, 2012; Riper et al., 2008). It is noteworthy that the control group also achieved a small reduction in alcohol intake and an increase in the percentage of respondents adhering to the guideline. This finding is in line with previous studies, which also found effects in control groups regarding alcohol intake (e.g. Boon et al., 2011) as well as regarding other lifestyle behaviours, such as physical activity (Bodner, 2008). Assessment alone can already have significant effects on drinking. The act of completing an assessment questionnaire may have induced the participants in the control group to monitor and reflect on their own behaviour, leading to a decrease in consumption (Bewick et al., 2010). 
Regarding our secondary goal - comparing an alternating and a summative tailoring strategy - we found no difference between the two strategies for changes in alcohol use. These results were the same among complete cases and ITT. Because both experimental subgroups ultimately received the same advice, the timing of the message delivery does not appear to have influenced behavioural impact. The attrition rates of our intervention show that more respondents in the alternating group completed the intervention at baseline. These respondents may have felt rewarded by receiving the advice in-between answering the questions, and this strategy may have made the programme more attractive. This was partly confirmed by the programme evaluation because the alternating group indicated having read more pieces of the personal advice. At six-month follow-up, however, the dropout rate no longer differed between the two experimental subgroups. The appreciation of the programme was also comparable between these two groups. Respondents who did not revisit the programme after six months were those who evaluated the programme more negatively at baseline, implying selective dropout.

\section{Strengths and limitations}

Our study was characterized by some strengths. First of all, our intervention programme was theorybased. The intervention satisfied the five basic criteria (i.e. the 5 A's) of the HBC-I (Evers et al., 2003; Evers et al., 2005) in addition to providing other essential tailoring elements. The respondents' answers to a number of questionnaires were used to give advice about the risks of heavy drinking and about the need to change their drinking behaviour; we assessed various possible predictors of behavioural change, such as attitude, social influence, self-efficacy, and planning; we assisted respondents by giving personal advice on the various psychosocial variables, including support and understanding, as well as personal information regarding relapse prevention (anticipatory guidance); and we arranged follow-up sessions. In previous research, extensive use of theory, including the Theory of Planned Behaviour (Ajzen, 1991), has been associated with considerable effects on healthrelated behaviour (Webb et al., 2010). Our programme was based on the I-Change model, which consists of the Theory of Planned Behaviour constructs supplemented by concepts such as awareness factors and action planning strategies. The latter, in particular, is associated with increased behavioural effects, as has been demonstrated in general (Guillaumie et al., 2012; Van Osch et al., 2008) as well as specifically for computer-tailored programmes (Elfeddali et al., 2012b). Our intervention programme used multiple tailoring by offering three feedback moments. A multi-session programme is likely to be more effective than a single-session programme (Brug et al., 1998; Dijkstra et al., 1998c; Elfeddali et al., 2012a). Further research should explore the optimal number of feedback moments as well as the optimal time lag between the different sessions. To our knowledge, this is the first study to compare two different tailoring strategies (i.e. alternating versus summative) in terms of effectiveness, dropout, and appreciation. Finally, few studies have tested a web-based tailored alcohol intervention among adults in the general population (A.B. Hansen et al., 2012).

Our study was also subject to some limitations. First, our findings were based on self-reports, which may have led to recall bias. Previous research has shown that quantity-frequency measures, such as 
those we used in this study, are likely to result in greater underestimation than daily diaries (Sobell et al., 1982). However, because we used the same questions at all measurement moments, this may have not influenced our data indicating changes in behaviour, and thus the effectiveness of the intervention. In any case, forgetting seems to be a potent source of underestimation in surveys regarding alcoholic drinks (Lemmens et al., 1992). Second, all respondents were recruited through an online panel and received an incentive for their participation, which might mean that some of them were not motivated to change their drinking behaviour and/or that they took part in this study simply to receive the incentive. Third, our study had a moderate-sized sample and a high attrition rate (approximately 41\%) as well as missing values on some baseline data (approximately 26\%). Based on the power analyses, the number of participants was sufficient for executing logistic regression analyses; however, for the linear regression analysis, our sample size was too small. Therefore, the effect among complete cases might have not reached statistical significance. However, the effect was found in the expected direction. Although our data still yielded statistically significant effects among the complete cases, it may be that a selective group (i.e. a very motivated group) completed the intervention programme. This implies that we have to interpret the results regarding the intervention effects of this subgroup carefully. However, further support was obtained in a sensitivity analysis employing LOCF. Thus, data analysis with complete cases and LOCF methods showed statistically significant intervention effects in reaching a low-risk drinking status and an effect of the intervention in decreasing the weekly amount of alcohol intake, although without reaching statistical significance. Analyses with multiple imputation methods did not confirm these findings. Although multiple imputation methods are regarded as the most preferred technique to handle missing data (Blankers et al., 2010), our analyses showed remarkable differences in outcomes. Moreover, the use of multiple imputation techniques may result in unreliable estimates when the number of missing values is high (White et al., 2011), as is the case in our study. Consequently, more research is needed to outline the conditions that yield these differences between the approaches. Additionally, when applying multiple imputation, the strategies how this technique is used should be clearly documented because multiple imputation techniques require certain procedures and rules (Allison, 2012; Bodner, 2008; White et al., 2011). At this moment, there seems to be no consensus about the number of imputed datasets needed (Allison, 2012; Blankers et al., 2010; Bodner, 2008; Graham et al., 2007; White et al., 2011). Other shortcomings of multiple imputation are that the results may strongly depend on the imputation model that is created (Sinharay et al., 2001; White et al., 2011) and that different multiple imputation programmes seem to show different results (Blankers et al., 2010). Finally, although we had a follow-up measurement six months after baseline, the long-term impact of web-based tailored interventions still remains unclear and requires further research.

\section{Conclusions}

Tailored feedback delivered via the Internet can be an effective way to reduce alcohol intake among adults, at least among a subgroup that revisited the programme. However, the results of complete case analyses were inconsistent with the findings of ITT analyses when using the multiple imputation 
technique. Among our Internet panel, there were no indications that an alternating or a summative tailoring strategy works better in reducing alcohol intake by means of eHealth programmes. Nevertheless, lower attrition rates during the first visit indicate that the version of the intervention with alternating questions and advice may be preferred. 



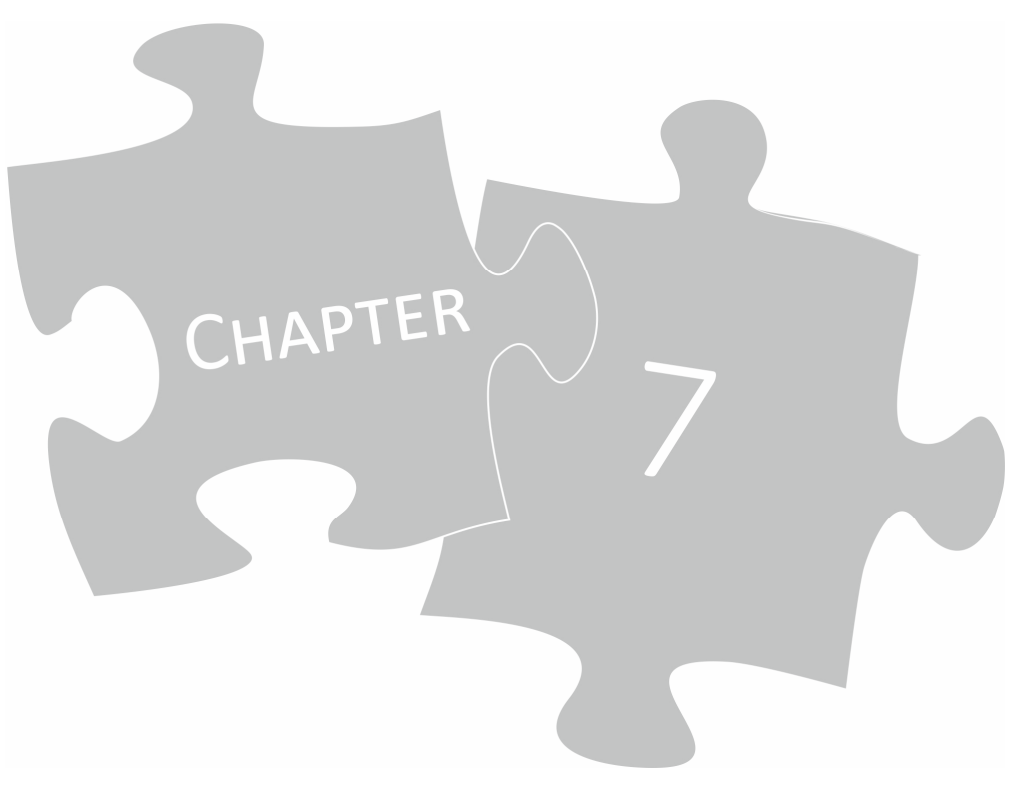

EFFECTS OF A WEB-BASED TAILORED MULTIPLE LIFESTYLE INTERVENTION FOR ADULTS: A TWO-YEAR RANDOMIZED CONTROLLED TRIAL COMPARING SEQUENTIAL AND SIMULTANEOUS DELIVERY MODES

This chapter has been published as:

Schulz DN, Kremers SPJ, Vandelanotte C, van Adrichem MJG, Schneider F, Candel MJJM, de Vries H. Effects of a web-based tailored multiple lifestyle intervention for adults: a two-year randomized controlled trial comparing sequential and simultaneous delivery modes. Journal of Medical Internet Research 2014; 16:e26. 


\section{ABSTRACT}

Background: Web-based computer-tailored interventions for multiple health behaviours can have a significant public health impact. Yet, few randomized controlled trials have tested this assumption.

Objective: To test the effects of a sequential and simultaneous web-based tailored intervention on multiple lifestyle behaviours.

Methods: A randomized controlled trial was conducted with three tailoring conditions (i.e. sequential, simultaneous, and control conditions) in the Netherlands in 2009-2012. Follow-up measurements took place after 12 and 24 months. The intervention content was based on the I-Change model. In a health risk appraisal, all respondents $(N=5,055)$ received feedback on their lifestyle behaviours that indicated whether they complied with the Dutch guidelines for physical activity, vegetable consumption, fruit consumption, alcohol intake, and smoking. Participants in the sequential ( $n=1,736)$ and simultaneous $(n=1,638)$ conditions received tailored motivational feedback to change unhealthy behaviours one at a time (sequential) or all at the same time (simultaneous). Mixed model analyses were performed as primary analyses; regression analyses were done as sensitivity analyses. An overall risk score was used as outcome measure, then effects on the five individual lifestyle behaviours were assessed and a process evaluation was performed regarding exposure to and appreciation of the intervention.

Results: Both tailoring strategies were associated with small self-reported behavioural changes. The sequential condition had the most significant effects compared to the control condition after 12 months $(\mathrm{T} 1$, effect size $=0.28)$. After 24 months $(\mathrm{T} 2)$, the simultaneous condition was most effective (effect size $=0.18$ ). All five individual lifestyle behaviours changed over time, but few effects differed significantly between the conditions. At both follow-ups, the sequential condition had significant changes in smoking abstinence compared to the simultaneous condition ( $\mathrm{T} 1$ effect size $=0.31 ; \mathrm{T} 2$ effect size $=0.41$ ). The sequential condition was more effective in decreasing alcohol consumption than the control condition at 24 months (effect size $=0.27$ ). Change was predicted by the amount of exposure to the intervention (total visiting time: $\beta=-.06 ; p=.01$; total number of visits: $\beta=-.11$; $p<.001)$. Both interventions were appreciated well by respondents without significant differences between conditions.

Conclusion: Although evidence was found for the effectiveness of both programmes, no simple conclusive finding could be drawn about which intervention mode was more effective. The best kind of intervention may depend on the behaviour that is targeted or on personal preferences and motivation. Further research is needed to identify moderators of intervention effectiveness. The results need to be interpreted in view of the high and selective dropout rates, multiple comparisons and modest effect sizes. However, a large number of people were reached at low costs and behavioural change was achieved after two years.

Keywords: multiple behaviour change; web-based intervention; computer-tailoring; effectiveness; physical activity; fruit consumption; vegetable consumption; alcohol intake; smoking 


\section{INTRODUCTION}

Since the development of the Internet, several types of web-based interventions have been offered to the population to modify unhealthy lifestyle behaviours. An unhealthy lifestyle can be described as one that is not compliant with the guidelines for different prominent health risk behaviours, such as being insufficiently active, eating insufficient fruit and vegetables, drinking too much alcohol, and using tobacco (Australian Institute of Health and Welfare, 2002; Smeets, 2006). Unhealthy lifestyle habits are among the main causes of mortality and morbidity (World Health Organization, 2002). Noncommunicable chronic diseases, such as heart diseases, cancer, diabetes, and chronic respiratory diseases (Australian Institute of Health and Welfare, 2002), are associated with a limited number of common, modifiable health behaviours (Ezzati et al., 2003).

Given the high prevalence of unhealthy lifestyle habits (De Vries et al., 2008b; Fine et al., 2001; Poortinga, 2007; Schuit et al., 2002), it is reasonable to offer interventions that can be disseminated among large numbers of people at low cost (Griffiths et al., 2006). Because individuals with multiple unhealthy lifestyle behaviours are at the greatest risk of developing chronic diseases, leading to increased health care costs (Edington, 2001), web-based interventions with a focus on different lifestyle behaviours and integrated within one intervention seem to be an appropriate choice. To increase changes in health behaviour, tailored interventions using a computerized expert system to select the best fitting messages to generate personal relevant feedback messages have been developed (De Vries \& Brug, 1999). Although inconsistent findings are reported (Neville et al., 2009b; Ryan \& Lauver, 2002), many web-based computer-tailored interventions have proven to be an effective tool for improving health-related behaviours (e.g. De Vries et al., 2008a; Krebs et al., 2010; Smeets et al., 2007). Different interventions based on the I-Change model have shown positive results (Elfeddali et al., 2012a; Smit et al., 2012a; Van Stralen et al., 2009a). As shown by Webb et al. (2010), the use of theory results in larger effect sizes. Moreover, web-based computer-tailored interventions which contain relevant and attractive information adapted to the respondents' individual characteristics and needs have proven to be cost-effective (e.g. Smit et al., 2011; Smit et al., 2013) and have been evaluated more positively than general information (De Vries et al., 2008b).

It has been suggested that interventions that focus on multiple behaviours have a greater impact on public health than single-behaviour interventions (De Vries et al., 2008b; Prochaska et al., 2008). However, such interventions are more extensive and thus require more engagement, time, and effort from the respondent. There is limited and inconsistent evidence about how best to accomplish multiple behaviour change when using web-based computer-tailored interventions. One strategy is to intervene in a single behaviour at a time (sequential approach); another approach is to intervene in all health risk behaviours at the same time (simultaneous approach) (De Vries et al., 2008b; Hyman et al., 2007; King et al., 2013; Oenema et al., 2008; Schulz et al., 2011; Vandelanotte et al., 2005; Vandelanotte et al., 2007a; Vandelanotte et al., 2008). In earlier studies, no consistent findings were reported regarding the most effective strategy. For example, King et al. (2013) and Hyman et al. (2007) reported that their simultaneous intervention mode was superior to their sequential intervention mode. The first aimed at changes in diet and physical activity; the latter aimed at smoking cessation 
and improvements in diet and physical activity. A study by Vandelanotte et al. (2008), aimed at lowering fat intake and increasing physical activity, found no differences between the sequential and the simultaneous condition.

Focusing on different behaviours sequentially at different points in time is associated with less cognitive effort during the individual visits; however, respondents may experience lower levels of autonomy because of the limited choices during the individual visits (Markland \& Vansteenkiste, 2007; Zuckerman et al., 1978), and repeated participation is necessary to receive information about multiple behaviours. Intervening in all behaviours simultaneously has the advantage that respondents receive all relevant information during the first visit; however, such a programme may lead to ego-depletion by overwhelming respondents with too much information (Baumeister et al., 1998; Hagger et al., 2010) which may lead to a more negative feeling regarding the intervention and immediate dropout. In previous research, dose-response relationships have been found between exposure to an intervention (number and duration of exposures) and behaviour change outcomes (Rodondi et al., 2006; Tate et al., 2001). This may imply that repeated participation and thus repeated exposure to the web-based programme can be beneficial for realizing substantial behavioural change (Verheijden et al., 2007). Appreciation of the intervention may lead to increased use, which, in turn, may improve intervention effectiveness (Brouwer et al., 2008; Crutzen et al., 2011; Elfeddali et al., 2012a). Thus, both delivery modes have potential advantages and disadvantages that may influence their effectiveness, use, and the appreciation of the different types of interventions. Current evidence regarding the effectiveness of sequential and simultaneous delivery modes is inconsistent and to date none of these studies have tested the two delivery modes within an intervention targeting five different health behaviours.

In summary, randomized controlled trials assessing the effects, especially the long-term effectiveness (after more than one year) (Davies et al., 2012a), of computer-tailored multiple lifestyle interventions using different strategies (sequential versus simultaneous) among adults are scarce. Therefore, the main aim of the current study was to assess whether a multisession, web-based tailored lifestyle intervention was effective in enhancing multiple lifestyle behaviours (i.e. physical activity, vegetable consumption, fruit consumption, alcohol intake, and smoking) in the long term. First, potential differences in effects of lifestyle change in general were assessed between the sequential and simultaneous delivery mode and a control condition at 12-month and 24-month follow-up. Second, we evaluated whether there were differences between the three groups with regard to effects on adherence to individual guidelines for each of the five behaviours being assessed. As a tertiary objective, a process evaluation was executed by studying the influence of intervention exposure on effectiveness and by summarizing the appreciation of the intervention. 


\section{METHOD}

A detailed description of the study protocol has been published elsewhere (Schulz et al., 2011); therefore, a summary of study methodology and procedures is provided subsequently.

\section{Participants, procedure and study design}

We conducted a randomized controlled trial (Dutch Trial Register $=$ NTR2168), involving two experimental conditions and a control condition. The web-based intervention, focusing on unhealthy lifestyle behaviours in the general population, was conducted in the Netherlands from November 2009 to July 2012. Follow-up measurements took place 12 and 24 months after the first intervention visit. In the first instance, adult participants were recruited via four Dutch Regional Health Authorities that had conducted the quadrennial Adult Health Monitor 2009 of inhabitants of the provinces of North-Brabant and Zeeland ( $N=96,388$ ). This monitoring tool is used to assess general health (e.g. physical and mental health) and cover health-related topics (e.g. social and physical environment) among representative samples of the Dutch population (National Institute for Public Health and the Environment, 2005). Of the 41,155 (42.70\%) respondents who completed the Monitor, 24,215 (58.84\%) filled out the written version and 16,940 (41.16\%) filled out the online version (see also Schneider et al., 2013b). Our intervention was partly integrated in the online version of the Monitor. At the end of this web-based questionnaire, participants were invited to take part in our intervention study. They had to give informed consent and provide their e-mail address for participation and the handling of their data. Three weeks later, the study sample received an e-mail containing an invitation and a link to the intervention website. After one month, a reminder e-mail was sent to the individuals in the sample who had not responded to the first invitation. The website was also open to the general public, so it was also possible to register directly on the website without having to complete the Monitor. Randomization to one of the three study groups took place by means of a computer software randomization system. No block or cluster randomization was applied; the randomization was done at the individual level. The following inclusion criteria were established for this study: being between ages 18 and 65 years, having a computer with Internet access, having basic Internet literacy, and having a valid e-mail address. The study was approved by the Medical Ethics Committee of Maastricht University and the University Hospital Maastricht (MEC 09-3-016/NL27235.068.09).

\section{Intervention programme}

The intervention programme, called myHealthyBehaviour (Dutch: "mijnGezondGedrag"), was a webbased computer-tailored programme targeting adults. The main aim of the intervention was to motivate participants to enhance five health behaviours. The theoretical framework for the development of the intervention was the I-Change model (De Vries et al., 2003). 


\section{The health risk appraisal}

In the first part of the intervention, all groups received a health risk appraisal (HRA) in which the five behaviours were placed in the context of the following Dutch public health guidelines for each of the different behaviours: being moderately physically active for 30 minutes on at least five days a week (Hildebrandt et al., 2010), eating 200 grams of vegetables per day (The Health Council of the Netherlands, 2006), eating two pieces of fruit per day (The Health Council of the Netherlands, 2006), not drinking more than one (women) or two (men) glasses of alcohol a day (The Health Council of the Netherlands, 2006), and not smoking (The Dutch Institute for Healthcare Improvement, 2004). Figures with pictures of traffic lights were presented for every behaviour to indicate whether respondents met (green), almost met (orange) or did not meet (red) the guidelines.

\section{Sequential and simultaneous delivery modes}

The second part of the intervention was only available for the two experimental conditions: (1) the sequential condition (SeqC) and (2) the simultaneous condition (SimC). In this part, self-assessed questionnaires were used to measure the psychosocial concepts of the I-Change model. The feedback messages were, in turn, based on these questionnaires and were revealed on the respondents' computer screens immediately after the completion of the surveys. Respondents who did not comply with at least one guideline were invited to change the behaviour which they had not complied with. They were invited to complete additional questions regarding motivational constructs related to a chosen behaviour (sequential condition) or all relevant behaviours (simultaneous condition) within one or more modules of the intervention, respectively. Motivational feedback was given that was related to the relevant behaviours. Feedback on each person's perception of the pros and cons of the health behaviour (attitudinal feedback) was given as the first step of the programme, followed by feedback on perceived social influences as the second step. For example, information was given on how the social environment could help the respondent to live healthily. In the third step, the concept of preparatory planning was addressed. Feedback was provided on how to prepare for behaviour change, for example by planning to be physically active at fixed times, adding the plans to an agenda, trying new/different kinds of sports, having enough vegetables in stock at home, eating fruit or vegetables as a snack, and (for smokers) planning a quit date. In the final step, the focus was placed on how to cope with difficult situations to also increase self-efficacy. Several tailoring strategies were used in the feedback messages; for example, respondents were addressed by their name, normative and ipsative feedback were given (i.e. during re-visits current scores were compared to scores of previous visits, see Figure 7.1), graphs and bar charts were included, and a personal tone and empathy was applied.

It was a multi-session programme, in which respondents were encouraged to revisit the website on an unlimited basis. After one year, respondents in the sequential condition had the opportunity to choose a second module and to receive feedback on another lifestyle behaviour. Respondents in the 
simultaneous condition received feedback on all behaviours for which they did not meet the guideline simultaneously at baseline and after 12 months. When visiting the website in the meantime, the sequential group received feedback on the chosen behaviour at baseline or at 12-month follow-up, respectively; the simultaneous group received feedback on all unhealthy behaviours they reported at that moment. After 24 months, an invitation to complete the last follow-up questionnaire was sent to all respondents, followed by two reminders to increase the response rate.

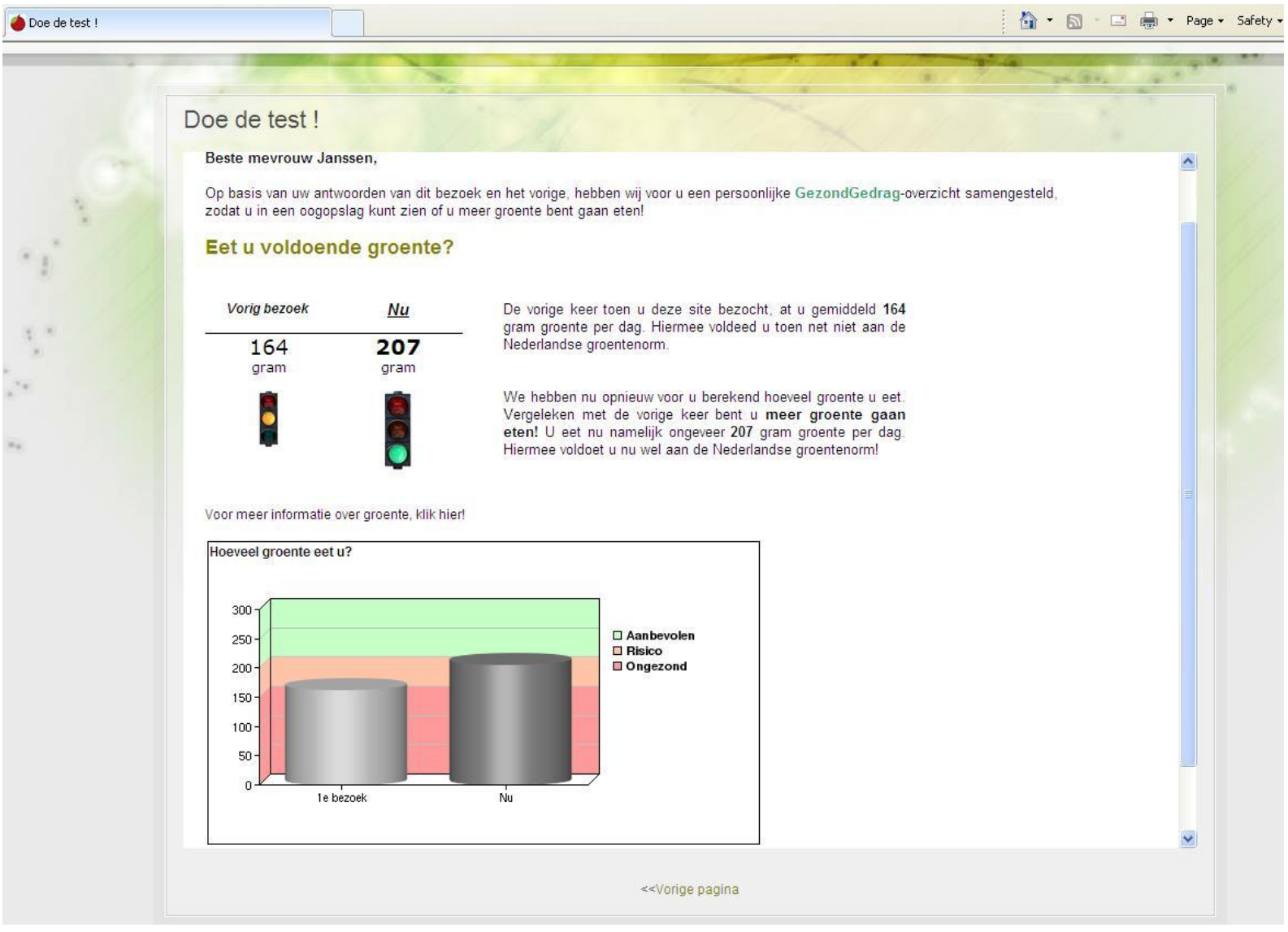

Figure 7.1 Screenshot of the intervention website showing the vegetable-part of the health risk appraisal

\section{Questionnaires}

\section{Demographic information}

We assessed eight demographic variables: age, gender (male $=1$; female $=2$ ), educational level (low = 1 , no education or primary education; medium $=2$, secondary education; high $=3$, tertiary education), net household income (euros per month), employment situation, marital status, number of persons in household, and native country (The Netherlands $=1$; other country $=2$ ). 


\section{Health status}

We assessed different kinds of diseases, such as cardiovascular diseases, diabetes, cancer, and high blood pressure. The 12-item short form (SF-12) Health Survey (Ware et al., 1995; Ware et al., 1996) was used to assess quality of life. The Kessler Psychological Distress Scale (K10) (Kessler \& Mroczek, 1994) was included to assess symptoms of depression and anxiety. The items regarding height and weight were used to estimate the body mass index (BMI).

\section{Lifestyle behaviour}

Physical activity was measured by the Short QUestionnaire to ASsess Health-enhancing physical activity (SQUASH) (National Institute for Public Health and the Environment, 2005; Wendel-Vos et al., 2003), and guideline adherence was assessed using comparable procedures developed by Ainsworth et al. (2000).

Fruit consumption was measured using a four-item Food Frequency Questionnaire (FFQ) assessing weekly fruit and fruit juice intake (National Institute for Public Health and the Environment, 2005).

Vegetable consumption was measured using a four-item FFQ assessing the weekly consumption of boiled or baked vegetables, as well as salads or raw vegetables (National Institute for Public Health and the Environment, 2005).

Alcohol intake was measured by the five-item Dutch Quantity-Frequency-Variability (QFV) questionnaire (Lemmens et al., 1992).

Current smoking behaviour was assessed by asking participants if they smoked, what they smoked (cigarettes, cigars, or pipe tobacco) and how much they smoked per day (cigarettes) or per week (cigars or pipe tobacco) (National Institute for Public Health and the Environment, 2005). The answers were converted into an overall score for tobacco consumption (expressed in number of cigarettes): one cigar equalled four cigarettes and one package of pipe tobacco ( 50 grams) equalled 50 cigarettes (Mudde et al., 2006).

\section{Social-cognitive variables}

Based on earlier studies (De Vries et al., 2008b; Smeets et al., 2007), the following social-cognitive variables were assessed regarding one or more of the five different lifestyle behaviours (dependent on the study condition). These variables were used to compose the personalized advice:

Intention was assessed by one item per behaviour using an extended version of the stage of change concept (Prochaska \& DiClemente, 1983; Prochaska et al., 1997). For example, for the question "Do 
you intend to be physically active for 30 minutes on at least five days a week?" answers included no, I don't intend to do so $(=1)$; I never thought about it $(=2)$; I thought about it, but I don't know yet $(=3)$; yes, but not within the next five years $(=4)$; yes, within one to five years $(=5)$; yes, within six to 12 months $(=6)$; yes, within three to six months $(=7)$; yes, within one to three months $(=8)$; yes, within a month (= 9); yes, and I'm already doing so $(=10)$.

Attitude was assessed by three pros per behaviour, such as "regular physical activity is good for my health" with responses ranging from totally disagree $(=1)$ to totally agree $(=5)$; and by three cons, such as "regular physical activity costs a lot of time" from totally disagree $(=1)$ to totally agree $(=5)$.

Social influence was assessed by social norms (one item), social modelling (one item) and social support (one item), such as "According to the people of my direct environment..." with answers ranging from I certainly should smoke $(=1)$ to I certainly should not smoke $(=5)$; "How many people in your direct environment smoke?" with answers ranging from nobody $(=1)$ to everybody $(=5)$; and "People from my direct environment support me not to smoke" with answers ranging from yes, they support me a lot $(=4)$ to no, they do not support me at all $(=1)$.

Preparatory plans were assessed by three items per behaviour, such as "I intend to allow time for physical activity" with answers ranging from no, definitely not $(=1)$ to yes, definitely $(=5)$.

Self-efficacy was assessed by six items per behaviour regarding difficult social, emotional, and routine situations, such as "I am able to meet the alcohol guideline ... when I am at a party; ...when I feel stressed or nervous; ...during a meal", or such "I am able to eat sufficient vegetables when I have other delicious food at home" with answers ranging from no, definitely not $(=1)$ to yes, definitely $(=5)$.

Coping plans were assessed by six items per behaviour, such as "I have made a plan to drink no more than two glasses of alcohol when I feel stressed or nervous" with answers ranging from totally disagree $(=1)$ to totally agree $(=5)$.

\section{Process evaluation}

To report user statistics, we recorded the number of logins to the tailored intervention per respondent and the time respondents spent on the tailored intervention during each visit. At baseline, after 12 months, and after 24 months, respondents evaluated the HRA on a scale from very bad (=1) to excellent $(=10)$. At 24-month follow-up, all respondents were invited to complete four more questions measuring appreciation of the programme by assessing the user-friendliness of the website and satisfaction with the layout, the HRA in general, and the use of traffic lights in particular. Additionally, a subsample completed a separate questionnaire, including items evaluating the website (six items), the HRA (13 items), and the personalized advice (15 items). 


\section{Power analyses}

Linear mixed model analysis (lifestyle factor is the outcome variable) was the main analysis and power analysis suggested that a total sample of 1,182 respondents was needed (correcting for possible attrition) based on $p=.05$, a power of $80 \%$, a two-sided test, and an effect size (ES) of 0.20 . For the logistic analyses, a sample of 882 respondents was needed.

\section{Statistical analyses}

The data were analysed using SPSS software, version 20 (IBM Corp, Armonk, NY, USA). To examine whether the randomization had been successful, the three groups were compared in terms of demographics, health status, and lifestyle behaviour; ANOVAs were executed for continuous variables and chi-square tests for discrete variables. In the case of significant differences, variables were included as covariates (i.e. potential confounders) in subsequent analyses.

Descriptive statistics were used to describe the characteristics of the study sample and the dropout rate within the groups. Dropout analyses, including a comparison between respondents lost to followup and TO-T1 completers (i.e. respondents who completed the baseline and the 12-month follow-up measurement) and TO-T2 completers (i.e. respondents who completed the baseline and the 24-month follow-up measurement), respectively, and fully complete cases were done using ANOVAs and chisquare tests.

We calculated a risk factor score by summing up all risky/unhealthy behaviours defined by the guideline status; the value for the risk factor score could range from 0 (adhering to all guidelines) to 5 (adhering to no guideline). The three study groups were compared in terms of their lifestyle behaviour at the follow-up measurements. First, ES were calculated based on means and odds ratios (Cohen's d). Those ES below 0.30 were considered small, whereas those between 0.30 and 0.80 were considered medium, and those larger than 0.80 were regarded as large (Cohen, 1988). Second, repeated measures analyses, using the top-down procedure, were conducted to study changes during the study period (time) and differences in changes between the study groups (time*condition). Linear mixed model analyses were used for the analyses with the risk factor score as outcome measure. Logistic mixed model analyses were used with the guidelines status for the five lifestyle behaviours as outcome measures. These kinds of analyses allow for inclusion of all cases (despite missing values of the outcome variable), and are valid in case the missing values satisfy the missingness at random assumption (Enders, 2010).

For the sensitivity analyses, differences in effect between the groups were explored by means of linear and logistic regression analyses by using the top-down procedure. The dependent variables were the risk factor score and complying with the physical activity guideline (yes $=1$; no $=0$ ), complying with the vegetable guideline (yes $=1$; no $=0$ ), complying with the fruit guideline (yes $=1$; no $=0$ ), complying with the alcohol guideline (yes = 1 ; no $=0$ ), and complying with the smoking guideline (yes = 1 ; no $=0$ ). All analyses were done for both the 12-month and 24-month follow-up measurement. To increase 
power and external validity, these regression analyses were first performed for T0-T1 completers and TO-T2 completers, respectively. Next, these analyses were also performed based on fully complete cases (i.e. respondents who completed both follow-up measurements). The results of the sensitivity analyses are outlined in the Appendices 3 and 4.

Among the experimental conditions, linear regression analyses were performed to study the predictive value of the total visiting time of the intervention and the total number of visits during the study period on the risk factor score after 24 months. Descriptive statistics were used to describe the evaluation/appreciation of the intervention at different time points.

Tests were performed at alpha $=.05$ for the intervention factor and alpha $=.10$ for covariates (Rosnow \& Rosenthal, 1989).

\section{RESULTS}

\section{Participation and sample characteristics}

Figure 7.2 shows the flow of the respondents from enrolment in the study to allocation to the three different conditions, and re-visits after 12 and 24 months. In total, 5,055 respondents were included in the analyses of this study, of which 4,833 (95.61\%) were participants of the Adult Health Monitor. A description of the study sample is shown in Table 7.1. We found some baseline differences between the three study groups. The age of respondents in the control condition was significantly higher when compared to the age in the sequential condition $(p=.03)$; more respondents in the experimental conditions suffered from heart attacks $(p=.01)$, but fewer people reported high blood pressure $(p=.002)$; compliance rates regarding vegetable intake were higher in the simultaneous group than in the control condition $(p=.07)$; and respondents in the sequential condition reported smoking more cigarettes than respondents in the control condition $(p=.04)$.

\section{Dropout analyses}

Respondents followed up and respondents lost to follow-up differed on a number of variables. Dropout was associated with some demographic factors (e.g. a younger age), a better health status as indicated by fewer diseases, and lower BMI, but an unhealthier lifestyle. More detailed information can be found in Appendix 2. 


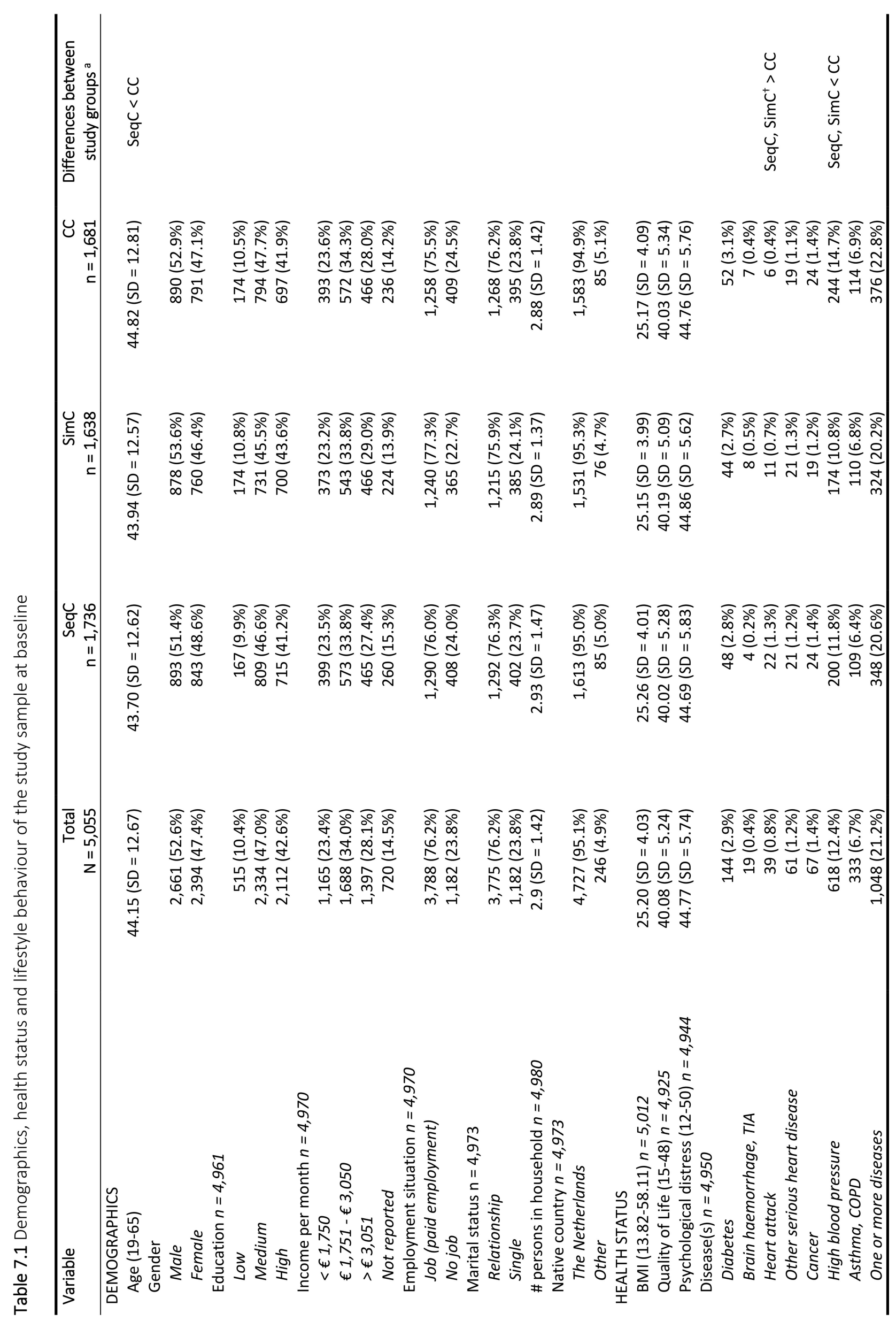




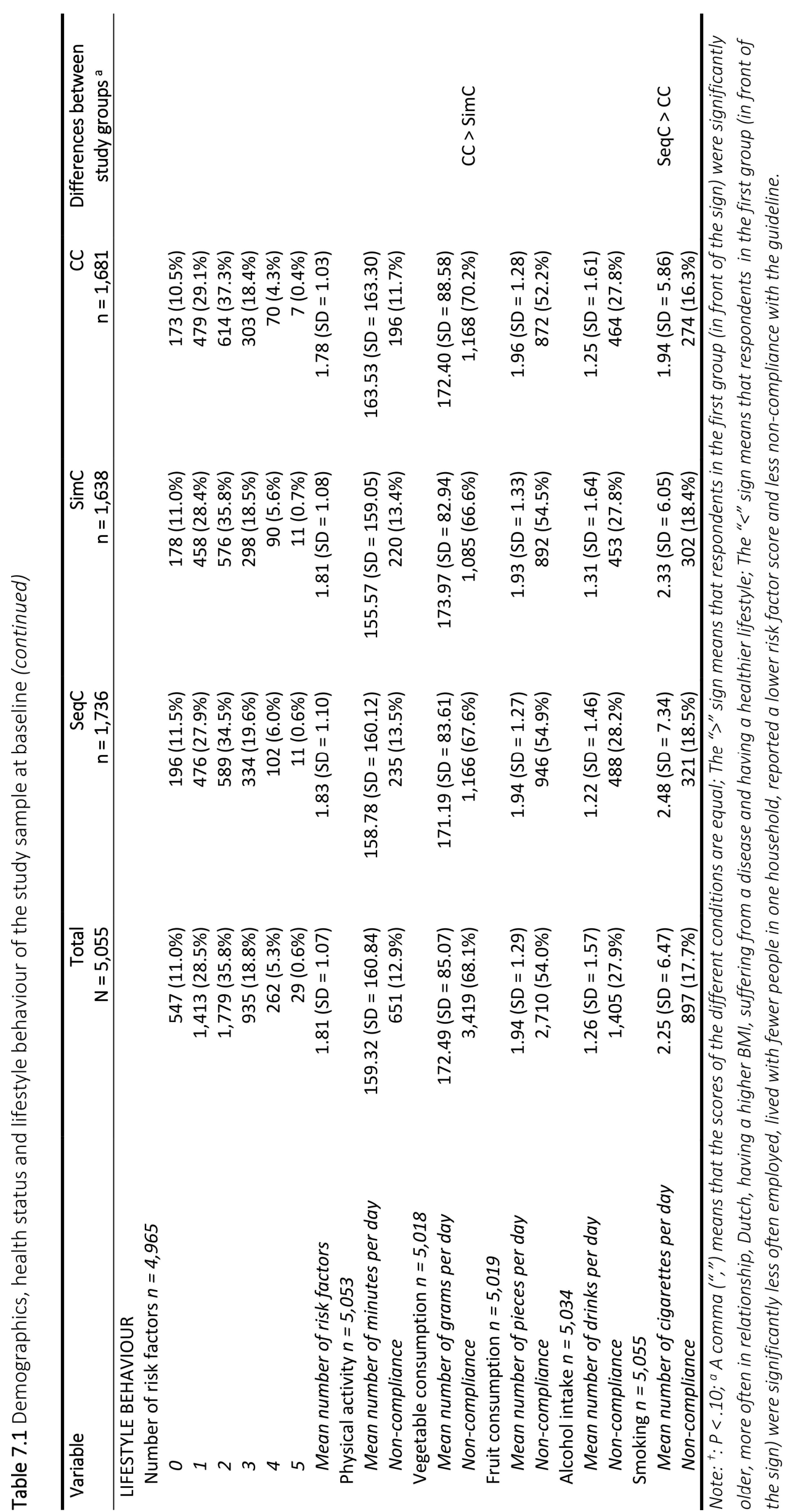




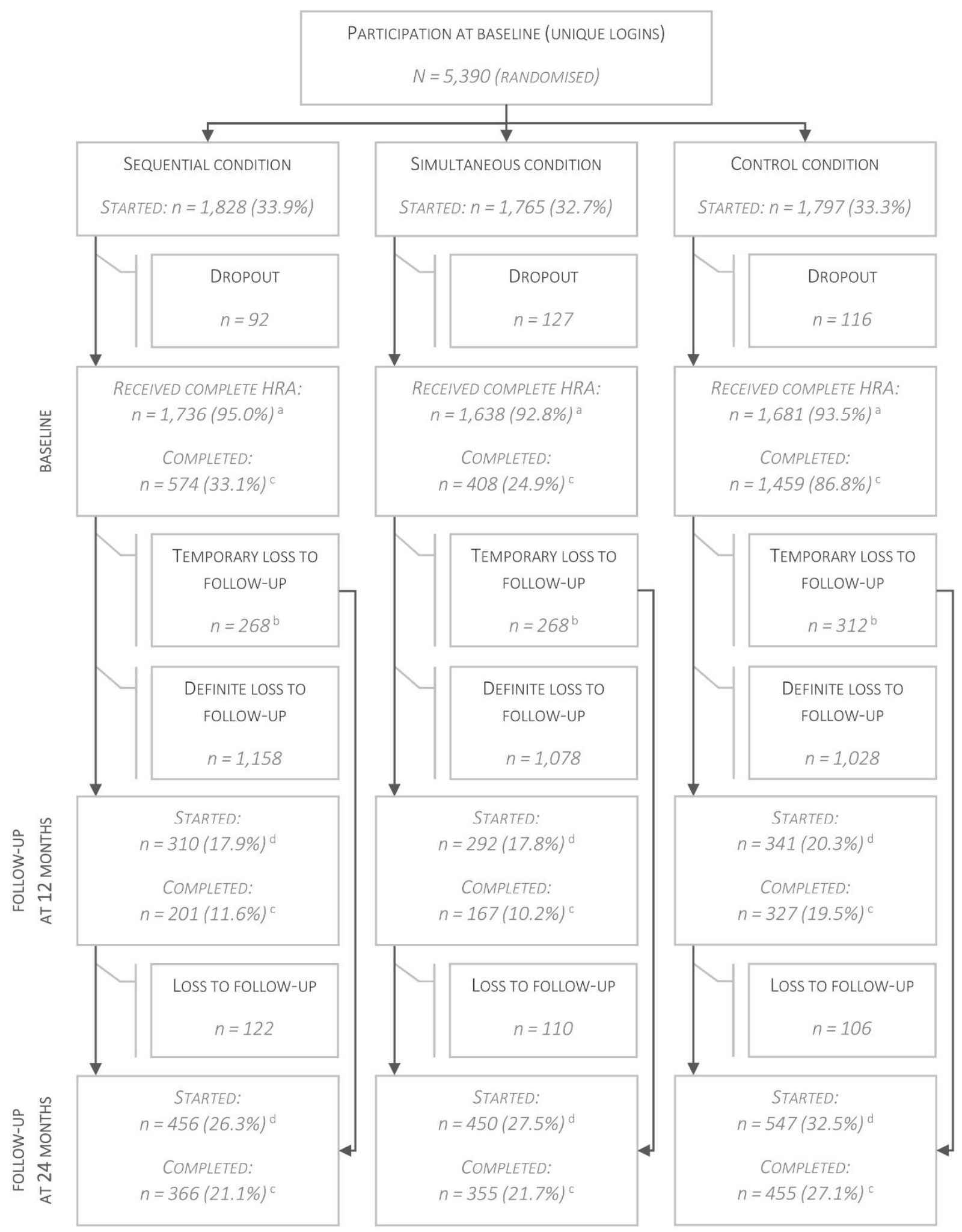

Figure 7.2 Flow-chart of study participants

Note: ${ }^{a}$ Analysed; ${ }^{b}$ Respondents who did not complete the follow-up at 12 month, but who did at 24 month; ${ }^{c}$ Respondents who adhered to all study protocols; ${ }^{d}$ Respondents who logged in to the intervention but did not complete the assessment. 


\section{Intervention effects}

We assessed the effects of the intervention on the overall lifestyle risk factor. The higher the score on the risk factor, the more a respondent did not comply with the Dutch guidelines concerning the lifestyle behaviours. The results of the linear mixed model analyses show that the risk factor score changed favourably and significantly over time (Figure 7.3) and that there is a statistically significant difference between the experimental conditions and the control condition (Table 7.2). After 12 months, the sequential condition was significantly more effective in reducing the risk factor score compared to the control condition. A similar, but not statistically significant effect $(p=.08)$ was found for the simultaneous condition compared to the control condition. After 24 months, only the simultaneous condition showed a statistically significant effect compared to the control condition, revealing a significantly lowered risk score for participants in the simultaneous condition. On both follow-up measurements, there were no statistically significant differences regarding the risk factor score between the sequential and the simultaneous condition.

\section{$\longrightarrow$ Sequential condition $\longrightarrow$ Simultaneous condition $\longrightarrow$ Control condition}

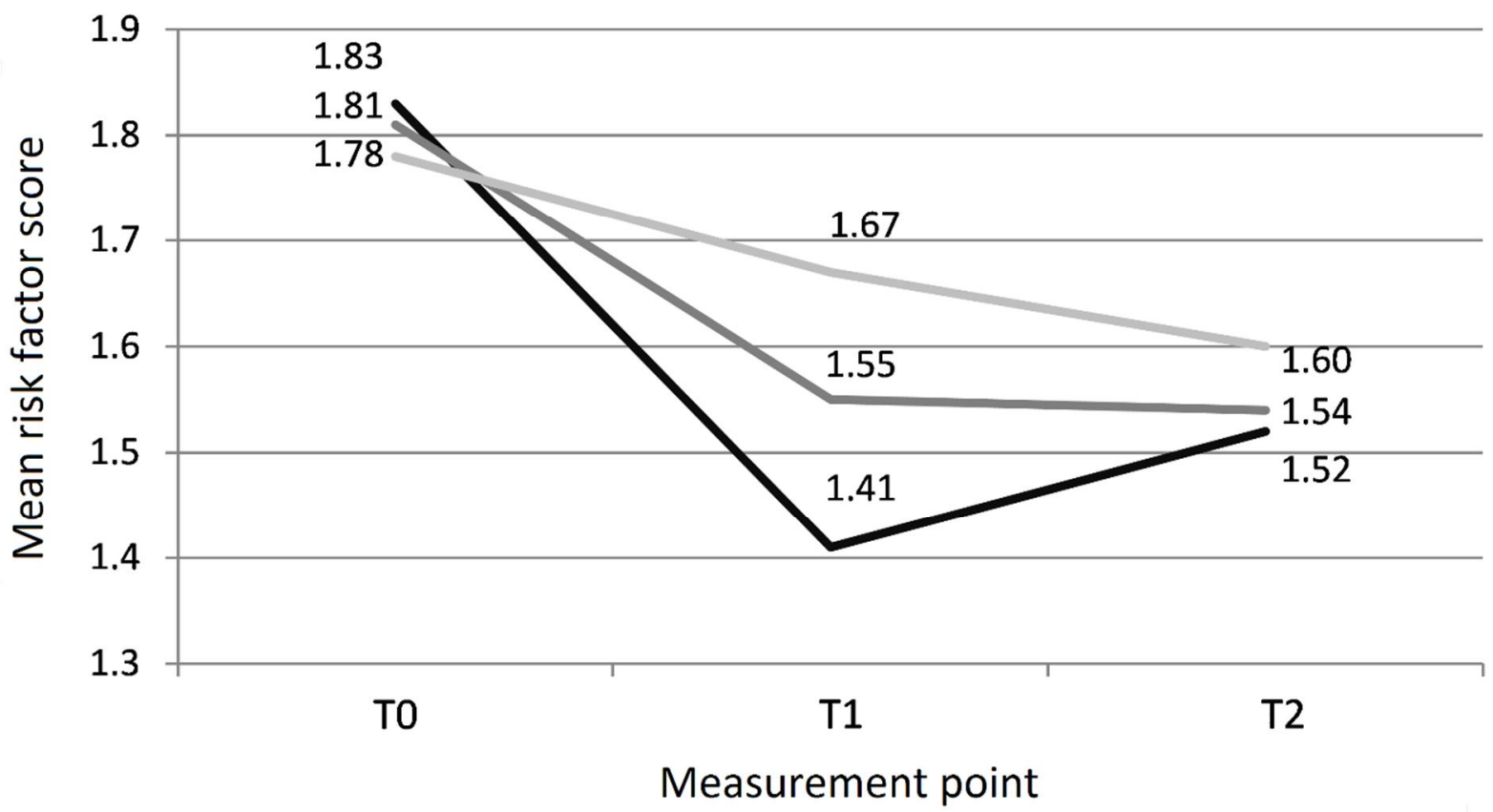

Figure 7.3 Mean number of risk factors among the different conditions at baseline (T0) and at 12 month (T1) and 24-month (T2) follow-ups

Table 7.2 Results of linear mixed model analyses (top-down procedure) ${ }^{\mathrm{a}}$ with the risk factor score after 12 months and after 24 months as outcome measure

\begin{tabular}{|c|c|c|c|c|c|c|c|c|}
\hline \multirow[b]{2}{*}{$\begin{array}{l}\text { Risk factor } \\
\text { score }\end{array}$} & \multicolumn{2}{|c|}{ Type III tests } & \multicolumn{3}{|c|}{ After 12 months (T1) } & \multicolumn{3}{|c|}{ After 24 months (T2) } \\
\hline & $\begin{array}{l}\text { Condition* } \\
\text { time }\end{array}$ & Time & $\begin{array}{c}\text { SeqC vs. } \\
\text { CC }\end{array}$ & $\begin{array}{c}\text { SimC vs. } \\
\text { CC }\end{array}$ & $\begin{array}{c}\text { SeqC vs. } \\
\text { SimC }\end{array}$ & $\begin{array}{c}\text { SeqC vs. } \\
\text { CC }\end{array}$ & $\begin{array}{c}\text { SimC vs. } \\
\text { CC }\end{array}$ & $\begin{array}{c}\text { SeqC vs. } \\
\text { SimC }\end{array}$ \\
\hline$p$ & .04 & $<.001$ & .008 & .08 & .39 & .13 & .048 & .68 \\
\hline ES & --- & --- & 0.28 & 0.19 & 0.10 & 0.14 & 0.18 & 0.04 \\
\hline
\end{tabular}

Note: ${ }^{a}$ All variables regarding demographics, health status, and lifestyle behaviour were included in the most extensive model. 
The sensitivity analyses showed similar results (see Appendices 3 and 4), except for two differences: (1) among the TO-T1 completers, both simultaneous and sequential interventions were effective in reducing the risk factor score after 12 months compared to the control condition, and (2) among fully complete cases, both simultaneous and sequential interventions revealed significant effects in reducing the risk factor score in comparison to the control condition after 24 months.

\section{Differences in lifestyle behaviours}

We conducted in-depth analysis to assess the effects of the interventions for each lifestyle behaviour separately. Table 7.3 summarizes the changes in the five different health risk behaviours according to the guideline status. The logistic mixed model analyses showed that all five lifestyle behaviours changed over time, but only a few effects differed significantly between the conditions. At both follow-up measurements, the sequential condition was found to result in significant changes in smoking abstinence in comparison to the simultaneous condition. After 24 months, the sequential condition had greater effect than the control condition, although this did not meet statistical significance $(p=.06)$. After 24 months, the sequential condition was more effective in decreasing alcohol consumption than the control condition. The differences between both conditions, although not statistically significant $(p<.10)$, give an indication that the simultaneous condition might have been more effective in increasing the amount of physical activity compared to the sequential condition after 12 months, and in increasing the fruit intake (after 12 and 24 months) compared to the control condition.

Table 7.3 Results of the logistic mixed model analyses (top-down procedure) ${ }^{a}$ with the specific lifestyle behaviour (guideline status) after 12 months and after 24 months as outcome measure

\begin{tabular}{|c|c|c|c|c|c|c|c|c|}
\hline \multirow[b]{2}{*}{$\begin{array}{l}\text { Outcome } \\
\text { measure }\end{array}$} & \multicolumn{2}{|c|}{ Fixed effects } & \multicolumn{3}{|c|}{ After 12 months (T1) } & \multicolumn{3}{|c|}{ After 24 months (T2) } \\
\hline & $\begin{array}{l}\text { Condition* } \\
\text { time }\end{array}$ & Time & $\begin{array}{c}\text { SeqC vs. } \\
\text { CC }\end{array}$ & $\begin{array}{c}\text { SimC vs. } \\
\text { CC }\end{array}$ & $\begin{array}{c}\text { SeqC vs. } \\
\text { SimC }\end{array}$ & $\begin{array}{c}\text { SeqC vs. } \\
\text { CC }\end{array}$ & $\begin{array}{c}\text { SimC vs. } \\
\text { CC }\end{array}$ & $\begin{array}{c}\text { SeqC vs. } \\
\text { Simc }\end{array}$ \\
\hline \multicolumn{9}{|c|}{ Physical activity } \\
\hline$p$ & .30 & .04 & .23 & .52 & .07 & .43 & .48 & .94 \\
\hline ES & --- & --- & 0.28 & 0.17 & 0.45 & 0.18 & 0.16 & 0.02 \\
\hline \multicolumn{9}{|l|}{ Vegetable } \\
\hline $\mathrm{p}$ & .65 & .046 & .26 & .41 & .78 & .62 & .66 & .37 \\
\hline ES & --- & --- & 0.20 & 0.15 & 0.05 & 0.07 & 0.07 & 0.14 \\
\hline \multicolumn{9}{|l|}{ Fruit } \\
\hline $\mathrm{p}$ & .21 & .13 & .61 & .06 & 18 & .30 & .07 & .46 \\
\hline ES & --- & --- & 0.08 & 0.29 & 0.21 & 0.14 & 0.24 & 0.10 \\
\hline \multicolumn{9}{|l|}{ Alcohol } \\
\hline$p$ & .27 & $<.001$ & .66 & .24 & .49 & .048 & .20 & .49 \\
\hline ES & --- & --- & 0.07 & 0.19 & 0.12 & 0.27 & 0.17 & 0.10 \\
\hline \multicolumn{9}{|l|}{ Smoking } \\
\hline$p$ & .045 & $<.001$ & .42 & .23 & .048 & .06 & .26 & .004 \\
\hline ES & --- & --- & 0.13 & 0.18 & 0.31 & 0.27 & 0.15 & 0.41 \\
\hline
\end{tabular}


However, when performing the sensitivity analyses, no consistent pattern could be found. Differences in statistical significance were found, especially regarding comparisons between groups with regard to fruit and vegetable intake, alcohol consumption, and smoking. More information can be found in Appendices 3 and 4 .

\section{Process evaluation}

\section{Exposure to the intervention}

When comparing the total visiting time and the total number of visits in the intervention during the 24 months, statistically significant differences between the three groups were found. On average, respondents in the sequential condition visited the intervention for 31 minutes $(S D=40)$, respondents of the simultaneous condition stayed on the website for 28 minutes (SD = 36); and respondents in the control condition visited the website for 16 minutes $\left(S D=21 ; F_{2,1124}=23.78\right.$; SeqC vs. $C C$ : $p<.001$; SimC vs. CC: $p<.001$; SeqC vs. SimC: $p=.31$ ). The mean number of visits in the sequential condition was 2.04 ( $S D=1.35)$; in the simultaneous condition this was 2.01 (SD = 1.45); and in the control condition this was 1.85 (SD $=0.93 ; F_{2,1124}=2.84$; SeqC vs. CC: $p=.75$; SimC vs. CC: $p=.16$; SeqC vs. SimC: $p=.91)$. The regression analyses conducted among respondents in the sequential and the simultaneous conditions only showed that the risk factor after 24 months was predicted by the total visiting time $(\beta=-.06 ; p=.01)$ and the total number of visits during the study period $(\beta=-.11 ; p<$ $.001)$. Longer visits and a greater number of visits predicted more favourable risk factor changes.

\section{Appreciation of the intervention}

The HRA was evaluated at all three measurement points. At baseline, the HRA score was evaluated as mean 7.2 ( $S D=1.3 ; n=2,441)$; after 12 months the HRA was evaluated as mean 7.3 (SD = 1.1; $\mathrm{n}=368)$; and after 24 months it was evaluated as mean $7.6(S D=1.0 ; n=1,176)$. No differences existed between the experimental conditions; however, at baseline, the HRA was more positively evaluated by the sequential condition (mean $=7.4 ; \mathrm{SD}=1.1 ; \mathrm{n}=574$ ) and the simultaneous condition ( mean $=7.3 ; \mathrm{SD}=1.4 ; \mathrm{n}=408$ ) than the control condition respondents (mean = 7.1; SD = 1.3; $\left.\mathrm{n}=1,459 ; \mathrm{F}_{2,2438}=16.48 ; \mathrm{p}<.001\right)$. Of those respondents who completed the last follow-up measurement, $84.43 \%$ ( $n=998$ ) stated that the HRA gave a good overview of their lifestyle, $77.58 \%$ $(n=917)$ liked the use of traffic lights in the HRA, $72.33 \%(n=852)$ liked the layout, and $76.74 \%$ $(n=904)$ experienced website use as user-friendly.

Additional evaluations of the intervention, filled out by 305 respondents, revealed no statistically significant differences between the conditions with regard to the website and the HRA. The evaluation of the tailored advice among respondents of the sequential and simultaneous condition revealed that the respondents evaluated the personalized advice as relevant $(75.4 \% ; n=86)$, credible $(76.5 \%$; $n=88)$, informative $(70.4 \% ; n=81)$, well-arranged $(84.3 \% ; n=97)$, clear $(85.1 \% ; n=97)$, interesting 
(71.3\%; $n=82)$, and with an attractive layout $(70.0 \% ; n=77)$. The personalized advice was evaluated as mean 6.9 (SD = 1.3), with no statistically significant differences between conditions. Almost $80 \%$ (79.8\%; $n=91)$ reported having read all parts of the advice, $40.9 \%(n=47)$ reported that they wanted to live a healthier life because of the received advice, and $16.5 \%(n=19)$ found the advice too long. One statistically significant difference was found $\left(\mathrm{t}_{111.49}=2.01 ; \mathrm{p}=.047\right)$ : respondents in the sequential condition indicated more often that they missed information in the advice than respondents in the simultaneous condition. Some suggestions were given for improving the intervention; for example, attention should be paid to personal circumstances by asking more specific questions about reasons for not eating sufficient vegetables or for being insufficiently physically active, and the advice could be made more personal by giving more concrete examples.

\section{DISCUSSION}

\section{Effectiveness of the intervention}

The primary aim of this study was to evaluate the effectiveness of two tailored web-based interventions compared to a control condition with regard to lifestyle improvement. Overall, the computer-tailored intervention for multiple health behaviours resulted in favourable lifestyle changes. Compared to the control condition, the sequential delivery mode was found to be most effective after 12 months, whereas the simultaneous delivery mode was most effective after 24 months. The sensitivity analyses yielded comparable results and suggested slightly stronger effects for both delivery modes, which may be because of those who were motivated to fill out more post-measurements were also those who were more motivated to change. The effect sizes were small which is common for tailored interventions, but they can still result in a large public health impact when widely implemented (Krebs et al., 2010; Lustria et al., 2013). For instance, for smoking cessation, a small ES is considered clinically significant (West, 2007) which may also be relevant for other behaviours addressed in our study. Moreover, our control condition received a minimal intervention (i.e. the tailored HRA). Personalized information regarding one's lifestyle behaviour may be sufficient to facilitate change and improve lifestyle behaviours (Parekh et al., 2009). Further studies are needed that compare a sequential and simultaneous condition for a group that receives general information or no information at all.

Regarding the overall lifestyle behaviour changes, no differences were found between the sequential and the simultaneous condition. This is in line with the findings Vandelanotte et al. (2008) reported in their study aimed at lowering fat intake and increasing physical activity. We assume that only those respondents with the highest motivational level to change remained in our study at follow-up. Therefore, it might be that no differential effects were found between the sequential and simultaneous condition.

When analyzing the separate behaviours, the largest changes were found for smoking cessation, followed by lower alcohol intake and increased fruit consumption. However, these findings were only 
partly replicated in the sensitivity analyses. The results of the sensitivity analyses supposed that the effect on the overall risk factor change can primarily be ascribed to changes in fruit consumption, vegetable consumption, alcohol intake, and probably in tobacco use. Hence, no firm conclusion can be drawn with regard to the differential effects on separate lifestyle behaviours. Further research is needed to investigate whether the optimum tailoring strategy (i.e. a sequential, simultaneous, or even single behaviour approach) may depend on the behaviour(s) being targeted.

\section{Dropout rates}

An important limitation of our study is the high dropout rates. Mixed model analyses were performed to increase internal and external validity; however, it is not unlikely that informative dropout occurred (i.e. the dropout process depends on the unobserved measurements) (Enders, 2010; Little \& Rubin, 2002). This would be a violation of the missing at randomness assumption underlying the mixed model analyses. The results of the sensitivity analyses, using regression techniques, might not be generalizable to the general population, but only to those people who remain participating in such a study over a longer period of time. As shown in our attrition analyses study (Schulz et al., 2012b), younger people and those with an unhealthier lifestyle were more likely to drop out.

We made use of a low-intensity implementation in the current study (recruitment via Regional Health Authorities, sending e-mails without face-to-face or telephone contact for the entire study). This implementation strategy may have led to relatively high attrition (Bennett \& Glasgow, 2009; Wantland et al., 2004). Yet, our dropout levels are comparable with those of other website-delivered studies with similar protocols (Eysenbach, 2005; Kosma et al., 2005; Woolf et al., 2006) and some participants indicated (via e-mail) that they did not revisit the intervention because they had complied with all five guidelines and found that they did not need more information. Furthermore, factors of the intervention itself may have caused dropout, such as technical issues or problems of navigating through the intervention website. As hypothesized in the Model of Internet Interventions, website characteristics, such as appearance, behavioural prescriptions, burdens, content, delivery, message, participation, and assessment may influence website use and effectiveness (Ritterband et al., 2009).

\section{Exposure rates}

There is growing evidence that the level of engagement in eHealth plays an important role in explaining the use and effectiveness of web-based interventions. It is challenging to develop eHealth interventions focused on multiple health behaviours that engage participants sufficiently to revisit the website on more than one occasion. As our results suggest, higher usage is related to higher effects, which has also been observed in other studies (Civljak et al., 2010; Danaher et al., 2008). With regard to the time respondents spent on the website, it is striking that many really short visits ( $<20$ seconds) were recorded. This is in line with the findings of Brouwer et al. (2010) who reported that more than half of their visitors left the website within 30 seconds. Internet interventions have the disadvantage 
that clicking off the website is easy. Respondents may have opened the link without knowing what they were opening (in our case: not reading the e-mail invitations to visit our intervention) resulting in loss of interest after opening the website. On the other hand, some quite long visits were recorded. The visiting time as recorded by the system may be not fully reliable, because it might be that respondents opened the website, but did not use it while it remained open. Moreover, visiting time also depends on the bit rate of the individual respondent's Internet.

In our intervention, exposure to the programme was similar for both the sequential and the simultaneous group, although respondents in the simultaneous condition had the possibility of receiving more parts of the programme than respondents in the sequential condition did. It may be that respondents in the simultaneous condition scanned the advice and did not read it carefully, resulting in comparable visiting times for the two intervention modes. In our sequential intervention, the modules were delivered sequentially over time so respondents could only choose one lifestyle module in the first year (Schulz et al., 2011). Exposure to multiple behaviours directly at the beginning of an intervention could have advantages for those people who drop out early (King et al., 2013). Those participating in this way will already have received more information during their first visit than those taking part in a single behaviour intervention in which the people who drop out after their first visit can only receive information about a single behaviour. Thus, it may be better to give respondents the choice of immediately initiating more than one module. A study by Brouwer et al. (2010) showed that more than half of their respondents selected two out of three modules and approximately one third initiated all three modules. A preference-based tailoring strategy (Kwak et al., 2007; Kwak et al., 2010) - a combination of our sequential and simultaneous condition - could be used in which respondents can select the modules they want to complete without any limitations on the number of behaviour modules. The finding that re-visits are uncommon in interventions (Vandelanotte et al., 2007b; Verheijden et al., 2007) - our respondents visited the intervention on average only twice - is a further argument against delivering the modules, or even sections of particular modules, sequentially over time.

\section{Strengths and limitations}

To our knowledge, this study is unique in comparing the effectiveness of sequential and simultaneous interventions in addressing the five lifestyle behaviours of physical activity, fruit consumption, vegetable consumption, alcohol intake, and smoking. The intervention was effective with a small ES, and was appreciated well. Our intervention met a number of criteria related to higher effectiveness of health behaviour change interventions offered via the Internet, such as extensive use of theory and behaviour change techniques (Webb et al., 2010). However, several limitations should be kept in mind: dropout rates were high resulting in a small sample size; the findings were based on self-reports which may have resulted in recall bias (e.g. the high proportion of respondents who reported being sufficiently physically active at baseline (87\%) may represent an overestimation of their actual level of physical activity; however, another reason for this high proportion might be that the intensity of the different kinds of physical activities was not measured in the short version of the SQUASH that we 
used (National Institute for Public Health and the Environment, 2005)); we cannot guarantee that a representative sample of the Dutch population was reached by our recruitment strategies; and a selective group filled out the follow-up questionnaires. Thus, the results may not be generalizable and may be biased. An additional limitation of our study is that respondents in the sequential condition had the possibility of choosing a maximum of two behavioural modules, whereas respondents in the simultaneous condition could receive personal feedback on more than two behaviours. Thus, the possible number of behaviour modules that could be completed differed between the sequential and the simultaneous group, which implies that the sequential condition might have been more effective if respondents had the opportunity to choose more than two modules. Finally, the control condition received a minimal intervention which might have led to improved lifestyle behaviours in this condition, too.

\section{Implications for future interventions and research}

The high prevalence of people engaging in multiple health risk behaviours calls for the development of multiple behaviour change interventions. Future studies should examine the number of behaviours that can be addressed in a multiple behaviour change intervention without overloading the respondents (Taylor et al., 2004). Research is needed to identify strategies to stimulate exposure and participation, in particular for Internet interventions with multiple sessions, such as those involving email/phone contact with visitors and updates of the intervention website (Brouwer et al., 2011; Crutzen et al., 2011; Schneider et al., 2013b). In addition to the necessity of research regarding the optimal delivery mode of these kinds of interventions, future research should focus on attracting, engaging, and retaining participants. In future interventions, other features could be added, such as short message service (SMS) text messaging, which can increase intervention effectiveness (Webb et al., 2010), or real-time sensors, such as accelerometer apps so that real-time motivational feedback can be provided (Vankipuram et al., 2012). Online community features, as a kind of self-help option, could be integrated in web-based lifestyle programmes to reduce attrition (Richardson et al., 2010). Moreover, interventions should be accessible on all different kinds of channels, such as desktop computers, laptops, smartphones and tablets. Research is needed to assess the additional effects of these elements.

\section{Conclusions}

Both sequential and simultaneous strategies were effective in improving lifestyle in a web-based computer-tailored intervention. Because no crucial differences have been found with regard to dropout rates and appreciation of the interventions, providers can use the strategy that suits their particular circumstances best. However, the best kind of intervention may be dependent on the behaviour that is targeted or other personal factors (e.g. motivational level to change). 



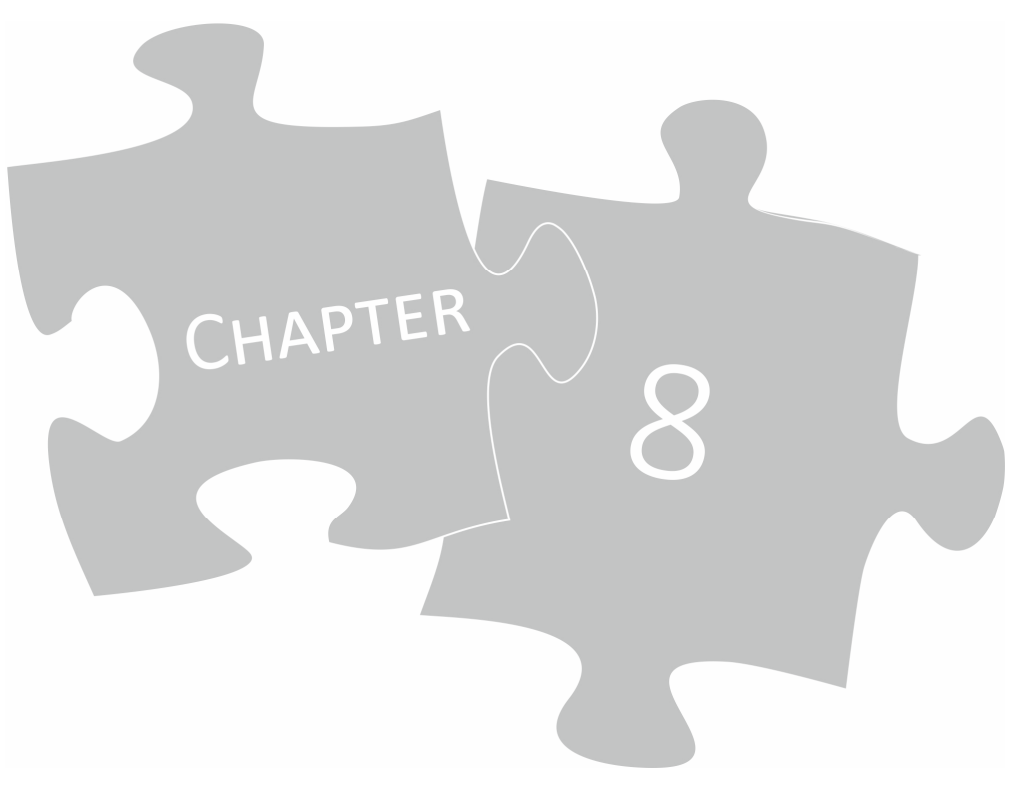

ECONOMIC EVALUATION OF A WEB-BASED TAILORED LIFESTYLE INTERVENTION FOR ADULTS: FINDINGS REGARDING COST-EFFECTIVENESS AND COST-UTILITY FROM A RANDOMIZED CONTROLLED TRIAL

This chapter has been published as:

Schulz DN, Smit ES, Stanczyk NE, Kremers SPJ, de Vries H, Evers SMAA. Economic evaluation of a webbased tailored lifestyle intervention for adults: findings regarding cost-effectiveness and cost-utility from a randomized controlled trial. Journal of Medical Internet Research 2014; 16:e91. 


\section{ABSTRACT}

Background: Different studies have reported the effectiveness of web-based computer-tailored lifestyle interventions, but economic evaluations of these interventions are scarce.

Objective: To assess the cost-effectiveness and cost-utility of a sequential and a simultaneous webbased computer-tailored lifestyle intervention for adults compared to a control group.

Methods: The economic evaluation, conducted from a societal perspective, was part of a two-year randomized controlled trial including three study groups. All groups received personalized health risk appraisals based on the guidelines for physical activity, fruit intake, vegetable intake, alcohol consumption, and smoking. Additionally, respondents in the sequential condition received personal advice about one lifestyle behaviour in the first year and regarding a second behaviour in the second year; respondents in the simultaneous condition received personal advice about all unhealthy behaviours in both years. During a period of 24 months, health care use, medication use, absenteeism from work and quality of life (EQ-5D-3L) were assessed every three months using web-based questionnaires. Demographics were assessed at baseline, and lifestyle behaviours were assessed at both baseline and after 24 months. Cost-effectiveness and cost-utility analyses were performed based on the outcome measures lifestyle factor (the number of guidelines respondents adhere to) and quality of life, respectively. We accounted for uncertainty by using bootstrapping techniques and sensitivity analyses.

Results: A total of 1,733 respondents were included in the analyses. From a willingness to pay of $€ 4,594$ per additional guideline met, the sequential intervention ( $n=552)$ was likely to be the most cost-effective, whereas from a willingness to pay of $€ 10,850$, the simultaneous intervention $(n=517)$ was likely to be most cost-effective. The control condition $(n=664)$ appeared to be preferred with regard to quality of life.

Conclusion: Both the sequential and the simultaneous lifestyle interventions were likely to be costeffective when it concerned the lifestyle factor, whereas the control condition was when it concerned quality of life. However, there is no accepted cut-off point for the willingness to pay per gain in lifestyle behaviours, making it impossible to draw firm conclusions. Further economic evaluations of lifestyle interventions are needed.

Keywords: randomized controlled trial; economic evaluation; cost-effectiveness; cost-utility; lifestyle behaviours; Internet interventions; web-based; computer-tailoring 


\section{BACKGROUND}

Non-communicable chronic diseases are associated with various modifiable health risk behaviours, such as physical inactivity, bad nutrition, excessive drinking, and smoking (Ezzati et al., 2002). An unhealthy lifestyle and the consequences involved are related to a reduced quality of life (Davies et al., 2012b) as well as substantial health care costs (Rappange et al., 2009; Von Lengerke \& Krauth, 2011). Stimulating a healthy lifestyle is important to improve health and prevent illness, and to also reduce health care costs, especially with current budget cuts in the Netherlands and other countries (Karanikolos et al., 2013; National Government, 2011).

Computer-tailoring can be used successfully as an intervention to promote behaviours associated with a healthy lifestyle (Krebs et al., 2010). When applying computer-tailoring, personalized feedback is generated by a computer programme based on an individual assessment (De Vries \& Brug, 1999). Earlier studies have demonstrated that tailored information is perceived as more relevant than nontailored information (Kreuter \& Wray, 2003). Moreover, computer-tailored interventions have proven to be effective in stimulating a healthier lifestyle, such as smoking cessation (Te Poel et al., 2009), preventing smoking relapse (Elfeddali et al., 2012a), encouraging healthy nutrition (Oenema et al., 2005), lowering alcohol intake (Riper et al., 2008), and increasing physical activity (Spittaels et al., 2007b). Previous research has also indicated that changing multiple lifestyle-related behaviours is likely to be more effective than changing only a single behaviour (Prochaska, 2008). A recent study has shown that tailored interventions that aim to reduce multiple health risk behaviours are not only successful in reducing unhealthy behaviours but also in simultaneously enhancing the overall wellbeing of the individual (Prochaska et al., 2012). The delivery of computer-tailored interventions targeting multiple health risk behaviours through the Internet has various benefits: These programmes can be applied in privacy and at a time and place the respondent finds convenient, many people can be reached at relatively low intervention costbecause more than $90 \%$ of the Dutch population has Internet access nowadays (Internet World Stats, 2012), and because the system is computerized it can be easily combined with, and/or integrated in other programmes or interventions.

Some economic evaluations of web-based and/or computer-tailored programmes have been conducted to date (e.g. Cobiac et al., 2009; Gerhards et al., 2010; Smit et al., 2011; Smit et al., 2013; Van Keulen et al., 2010a; Warmerdam et al., 2010). In general, these studies have given a first indication that these interventions - most were single behaviour change interventions - can indeed be cost-effective. To our knowledge, however, no economic evaluation of a web-based computer-tailored intervention targeting multiple health risk behaviours has been conducted so far.

Web-based computer-tailored lifestyle interventions are an interesting and promising option to make the healthcare system more sustainable, because of their proven clinical effectiveness and their potential cost-effectiveness due to relatively low intervention costs and wide reach. Thus, information regarding the cost-effectiveness of web-based computer-tailored intervention programmes is crucial for health care decision makers and the government, in making evidence-based decisions regarding large-scale implementation of such programmes (Tate et al., 2009). The aim of the present study, 
therefore, is to assess from a societal perspective the cost-effectiveness and cost-utility of two different versions (sequential and simultaneous) of a web-based computer-tailored lifestyle intervention for adults, compared to a control group that received only a minimal intervention.

\section{METHODS}

\section{Study design and participants}

The economic evaluation was embedded in a two-year single-blind randomized controlled trial, including three study groups. The study was approved by the Medical Ethics Committee of Maastricht University and the University Hospital Maastricht (MEC 09-3-016/NL27235.068.09) and registered by the Dutch Trial Register (NTR2168).

In October 2009, the Dutch Regional Health Authorities of North-Brabant and Zeeland conducted the quadrennial Adult Health Monitor 2009 among inhabitants of these two provinces. This questionnaire could be completed on paper or online via the Internet. Respondents who completed the online version of the questionnaire were invited to take part in the present study. The Monitor was interconnected with and integrated into our web-based lifestyle intervention. The study website was also open to the general public; therefore, it was also possible to register for participation in the trial directly on the study website without having completed the Adult Health Monitor.

The inclusion period for this study was from November 2009 up to and including July 2010. The following inclusion criteria were used: aged between 18 and 65 years, having a computer with Internet access and basic Internet literacy, and having a valid e-mail address. Participants were randomized into one of the two experimental groups (sequential condition (SeqC) or simultaneous condition $(\operatorname{SimC})$ ) or into the control condition (CC), with an equal probability of being assigned to any of the three groups. Randomization took place at the individual level by means of a computer software randomization system. Figure 8.1 shows the Consolidated Standards of Reporting Trials (CONSORT) flow diagram.

\section{Intervention}

The intervention was a web-based computer-tailored multi-session programme targeting adults. The primary aim of this lifestyle intervention was to motivate participants to be sufficiently physically active, to eat enough fruit and vegetables, to drink less alcohol and to quit smoking. The intervention was based on the I-Change model, an integration of social cognitive models (De Vries et al., 2003; De Vries et al., 2013), and previously developed programmes that have proven to be effective in increasing the level of physical activity (Smeets et al., 2008), promoting the intake of fruit and vegetables (Oenema et al., 2005), reducing the consumption of alcohol (Riper et al., 2008), and smoking cessation (Dijkstra et al., 1998b). The respondents received feedback texts on their computer 
screens which were aimed to motivate them to adopt the recommended health behaviours. All respondents received a health risk appraisal (HRA) indicating whether they adhered to the following public health guidelines: being moderately physically active for 30 minutes at least five days a week (Hildebrandt et al., 2010), eating 200 grams of vegetables per day (The Health Council of the Netherlands, 2006), eating two pieces of fruit per day (The Health Council of the Netherlands, 2006), not drinking more than one (for women) or two (for men) glasses of alcohol a day (The Health Council of the Netherlands, 2006), and not smoking (The Dutch Institute for Healthcare Improvement, 2004).

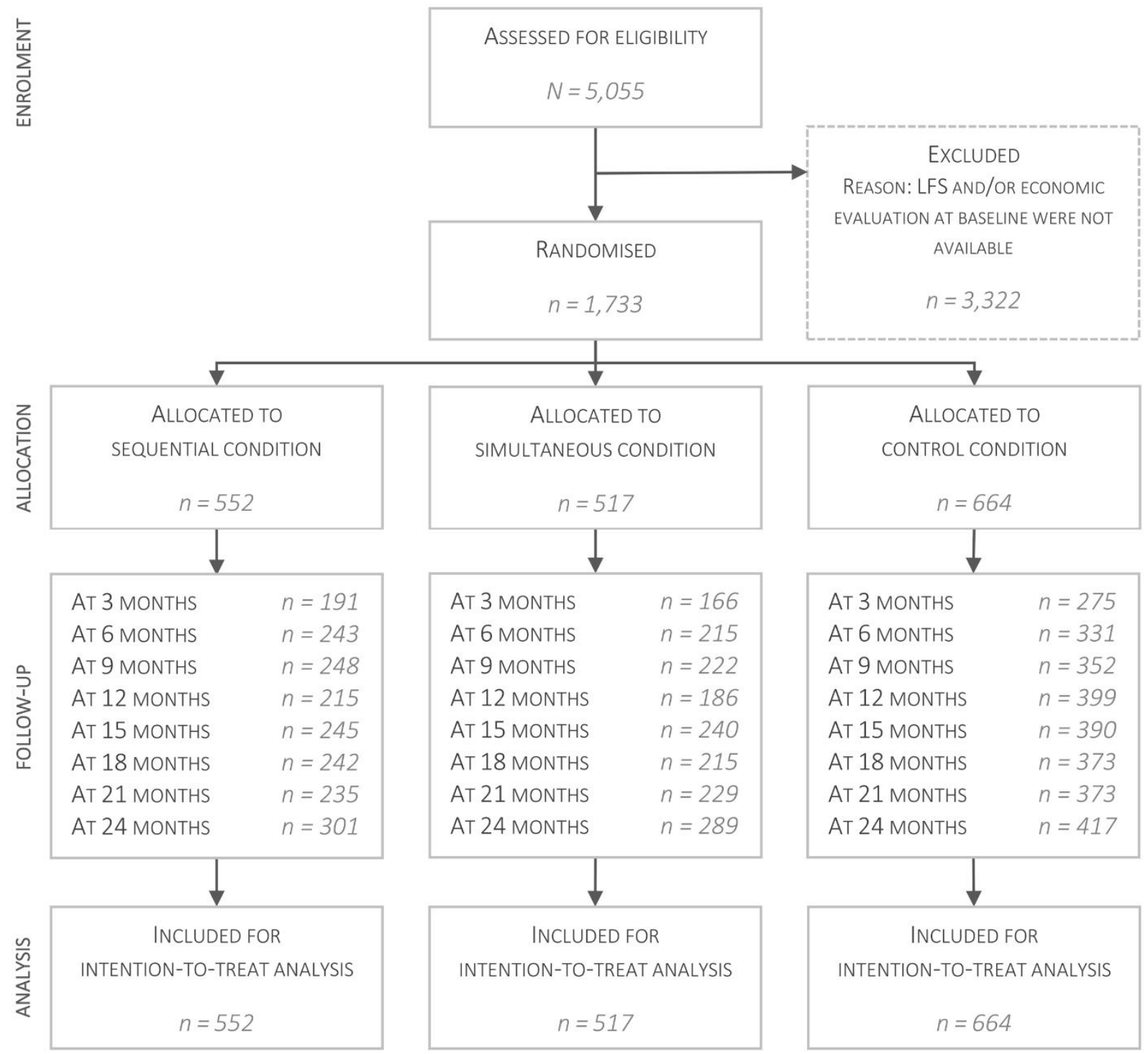

Figure 8.1 Flow diagram of the economic study

For all health risk behaviours, they received a traffic light indicating whether they met (green), almost met (orange) or failed to meet (red) the guideline. The experimental groups subsequently received personalized advice provided in four steps based on questions about different psychosocial 
determinants of the I-Change model (De Vries et al., 2003): (1) attitude, (2) social influence, (3) preparatory planning, and (4) self-efficacy and coping planning. At the end of every step, personal advice was given to participants. At baseline, respondents in the sequential condition could select a module concerning one of the lifestyle behaviours for which they did not meet the public health guidelines (i.e. received a red or orange traffic light in their HRA); on completing this module, they received personalized feedback regarding this particular behaviour. After 12 months, a second assessment took place and respondents had the opportunity to choose a second module and to receive feedback on a second lifestyle behaviour for which they did not meet the public health guidelines. At baseline and after 12 months, respondents in the simultaneous condition received feedback on all the behaviours they did not meet the public health guideline for at one time. In both conditions, an overview of all received pieces of advice was available (via a link which was also sent by e-mail) for the respondent at the end of the sessions. The control group received the HRA at baseline and after 24 months, but did not receive any additional personal advice. Figure 1.2 (see chapter 1) presents the design of the study, including all parts of the intervention. A detailed description of the study protocol has been published elsewhere (Schulz et al., 2011).

\section{Identification, measurement and valuation of costs and effects}

The economic evaluation was conducted from a societal perspective; therefore, all relevant costs (i.e. intervention costs, health care costs and respondent costs) and effects, such as Quality Adjusted Life Years (QALYS) and lifestyle factor score (LFS) were taken into account (Drummond, 1997).

\section{Costs}

Intervention costs consisted of hosting costs for the website, including costs for technical assistance and required updates. Costs for the development of the intervention programme and research specific costs were excluded, because these are once-only costs that are not necessary again when implementing the programme. The intervention costs were the same for all study groups because all groups received tailored advice that was integrated in the study website.

Health care costs included use of medication, medical consultations, inpatient and outpatient specialist care, hospital admissions, and other care (e.g. professional home care). Health care costs were assessed using a three-month retrospective questionnaire consisting of multiple choice and open-ended questions. This online questionnaire was taken quarterly during the 24 months. The updated Dutch Manual for Cost Analysis in Health Care was used to valuate costs (Hakkaart-Van Roijen et al., 2010). If cost-prices were not available, other sources were used. For instance, the website of the Healthcare Insurance Board (2013) was used to calculate medication costs. The costs of medications were calculated based on the dose described by the respondent. Hence, costs per tablet, gram or millilitre were used to calculate total medication costs for each respondent. Costs for health care services that could not be found in the Dutch Manual for Cost Analysis in Health Care were 
looked up on the Internet (e.g. via the websites of health care services). If possible, three costs for each health care service were looked up, to ultimately calculate a mean cost prize for this service. Cost price details can be found in Appendix 5.

Productivity costs included costs because of sickness absenteeism from work. They were calculated by the human capital method using mean costs for the Dutch population corrected for gender and age (Hakkaart-Van Roijen et al., 2010).

Respondent costs (also known as patient and family costs) included the time respondents spent on the website for participation, and costs for travelling to health care services. For the time spent on the website, we used the mean time that was necessary to complete the programme within the three study groups. The time lost due to participation in the Adult Health Monitor was also taken into account, but we only added the time people needed to fill out the parts of the Adult Health Monitor regarding the five lifestyle behaviours. We made this decision for two reasons: Firstly, it was not necessary to combine the interventions and the entire Monitor of the Regional Health Authorities; secondly, respondents who participated in the Adult Health Monitor skipped these parts on our website, whereas people who did not take part in the Adult Health Monitor completed these questions on our website (i.e. in the end, all respondents completed the same number of questions). Ultimately, we used an average time of 70 minutes for the sequential condition; 100 minutes for the simultaneous condition; and 20 minutes for the control condition. To determine the cost of time spent on the website, we valued the time by applying the labour time using the mean costs of the Dutch population corrected for gender and age (Hakkaart-Van Roijen et al., 2010). Costs for travelling to health care services were also valued in monetary terms. These costs were assessed based on average travel distances to health care services in the Netherlands (National Institute for Public Health and the Environment, 2008) and the mean costs per kilometre (Hakkaart-Van Roijen et al., 2010).

\section{Effects}

For the cost-effectiveness analysis, the primary outcome measure was the total LFS. The following questionnaires were used to assess the five lifestyle behaviours: the Short QUestionnaire to ASsess Health-enhancing physical activity (SQUASH) (National Institute for Public Health and the Environment, 2005; Wendel-Vos et al., 2003), a four-item Food Frequency Questionnaire (FFQ) assessing weekly fruit and fruit juice intake (National Institute for Public Health and the Environment, 2005), a four-item FFQ assessing the weekly consumption of boiled or baked vegetables, as well as salads or raw vegetables (National Institute for Public Health and the Environment, 2005), the fiveitem Dutch Quantity-Frequency-Variability (QFV) questionnaire to assess alcohol intake (Lemmens et al., 1992; National Institute for Public Health and the Environment, 2005), and questions asking participants if they smoked, what they smoked (cigarettes, cigars, or pipe tobacco) and how much they smoked per day (cigarettes) or per week (cigars or pipe tobacco) (National Institute for Public Health and the Environment, 2005). Based on the guidelines for physical activity, fruit intake, vegetable intake, alcohol consumption, and smoking, we calculated this LFS by summing up all healthy 
behaviours (i.e. complying with the guideline in question) - a similar method (Prudence score) was applied by Parekh et al. (2009) - at baseline and after 24 months; thus, the value of the LFS could range from 0 (adhering to no guidelines) to 5 (adhering to all guidelines). Moreover, a lifestyle factor change index (LFCI) was calculated by subtracting the LFS at baseline from the LFS at 24 months (Carlson et al., 2012). The value for this index could range from -5 to +5 on a continuous 11-point scale; positive scores indicated an increase, whereas negative scores indicated a decrease in the number of healthy behaviours.

For the cost-utility analysis, the primary outcome measure was utilities based on a health-related quality of life instrument. The Euro-Qol EQ-5D-3L questionnaire (EuroQol Group, 1990) was used to assess health-related quality of life and was completed by respondents every three months. The EQ5D-3L consists of the following five health dimensions: mobility, self-care, daily activity, pain/discomfort, and anxiety/depression. On a three-point Likert scale, respondents had to indicate their own state of health (no complaints $=1$; some complaints $=2$; many complaints $=3$ ). A utility score was calculated for each measurement point, using the Dutch tariff (Lamers et al., 2005). This score could range from -0.33 (death) to 1 (perfect health). This utility score, in turn, was used to calculate the QALYS gained or lost during the two-year study period by making use of the area under the curve method. The area under the curve stands for the duration of the health state (x-axis, 24 months) multiplied by the quality weight for the health state (y-axis; utility score).

\section{Indexing and discounting}

The price year of this study was 2013. All cost prices were indexed to the year 2013 using the consumer price indexes of 105.38 for the year 2009, 106.72 for the year 2010, 109.22 for the year 2011, 111.90 for the year 2012 and 115.00 for the year 2013 (Statistics Netherlands, 2013c). Because of the long-term follow-up of two years, costs made in the second year were discounted by $4 \%$, and effects regarding the LFS assessed after 24 months and effects in QALYs measured in the second year of the study were discounted by 1.5\% (Hakkaart-Van Roijen et al., 2010; Health Care Insurance Board, 2006).

\section{Statistical analyses}

Respondents were included in the analyses when their LFS at baseline was available and when the economic evaluation measurement was completed at least at baseline.

To examine whether randomization was successful and whether the three groups were comparable in terms of demographics, baseline LFS, quality of life and health care costs over the previous three months, analyses of variance (ANOVA) were used for continuous variables and chi-square tests for dichotomous or categorical variables. To investigate whether selective dropout had occurred, logistic regression analyses were used to compare (1) those who took part in the intervention, but did not 
complete the questionnaires needed for the economic evaluation, with those who did complete these questionnaires and were included in this study, and (2) of those included in this study, those who filled out at least one follow-up questionnaire regarding the economic evaluation, with those who did not fill out any of the follow-up questionnaires.

Intention-to-treat analyses were performed. Mean imputation was used to fill in missing values regarding medication use, health care services, absenteeism from work, EQ-5D-3L items and lifestyle items. When applying mean imputation, the mean of the previous and next value for the same variable was calculated. If mean imputation was impossible because of missing values on multiple measurement points, the last observation carried forward (LOCF) method was used to fill in missing values (Hendriks et al., 2013). The next observation carried backward method was used when the value was not available on the baseline questionnaire. Unrealistic/impossible values (e.g. more than 90 days absent from work in a period of 90 days) were recoded as the highest possible value. In case of unclear answers to the open ended question regarding medication use (e.g. private, too much, I do not know anymore), mean prices of the study group for this question were imputed.

To compare the three study groups regarding their biennial costs (i.e. health care costs and respondent costs over a period of two years), nonparametric bootstrapping (5,000 times) with 95\% confidence intervals in percentiles was used. ANOVAs were performed to compare the groups regarding the LFS assessed after 24 months and the QALYs measured over the study period of two years.

Incremental costs (in Euros) and effects were calculated for all three study groups as well as a net monetary benefit by valuing the effectiveness and utility outcomes in monetary values using a threshold for society's willingness to pay (WTP) per gain in the LFS (i.e. per additional guideline met) and per QALY gained (Stinnett \& Mullahy, 1998). The probability of the highest net monetary benefit was presented from a WTP of $€ 0$ to $€ 80,000$ (Council for Public Health and Care, 2006). Additionally, we explicitly reported the probabilities when using a WTP of $€ 18,000$ because this is an accepted Dutch cut-off point per QALY gained as a result of preventive interventions (Council for Public Health and Care, 2006).

\section{Uncertainty analyses}

For the cost-effectiveness and cost-utility analyses, bootstrapping resampling techniques (with 1,000 times replacement) were carried out to deal with uncertainty around the estimates of costeffectiveness and cost-utility. The results were presented in cost-effectiveness and cost-utility acceptability curves. Seven different sensitivity analyses were performed to deal with the uncertainty of parameter estimates from the primary analysis: (1) we executed the analyses from a health care perspective by excluding the productivity costs and the respondent costs (these might be reflected in participants' reported quality of life anyway) (Hakkaart-Van Roijen et al., 2010); (2) we excluded costs due to absenteeism from work because these costs differed significantly between the three study groups before the intervention; (3) we excluded respondents with less than four follow-up 
measurement points because of the large number of missing values (>50\%); (4) we used a LFCl as outcome variable to correct for the LFS before the intervention (cost-effectiveness analysis) and corrected the QALY for baseline utility (cost-utility analysis) (Manca et al., 2005); (5) we excluded respondents with the highest costs based on the 95 $5^{\text {th }}$ percentile; (6) we did not discount costs and effect outcomes (Hakkaart-Van Roijen et al., 2010; Health Care Insurance Board, 2006); and (7) we discounted both the costs and effect outcomes by $4.0 \%$ instead of discounting only costs by $4.0 \%$ and effects by 1.5\% (Health Care Insurance Board, 2006).

Bootstrap analyses were done using Microsoft Office Excel 2010; all other analyses were done using SPSS version 20.0 (IBM Corp, Armonk, NY, USA).

\section{RESULTS}

\section{Sample characteristics}

A total of 1,733 respondents were included in the analyses. Table 8.1 presents the baseline characteristics of the three study groups. One baseline difference was found between the groups: the sequential condition had significantly higher costs because of absenteeism from work compared to the control group.

\section{Dropout analyses}

Results from the logistic regression analysis showed that respondents who were excluded from the analyses of the present study (i.e. those who took part in the intervention, but did not complete the questionnaires needed for the economic evaluation; $n=3,322)$ were significantly younger $(B=0.03$; $p<.001)$, had a lower LFS $(B=0-.12 ; p<.001)$, reported less diseases, such as brain haemorrhage and transient ischemic attack $(T I A)(B=1.14 ; p=.04)$, and more of them had no paid job $(B=0.40$; $\mathrm{p}<.001$ ) than respondents included in our analyses.

Each of the eight retrospective questionnaires for the previous three months required for the economic evaluation was completed by at least $36.47 \%(632 / 1,733)$ and not more than $57.76 \%$ $(1,001 / 1733)$ of the respondents, whereas 506 of 1733 respondents $(29.20 \%)$ did not complete any of these questionnaires. The latter were younger $(B=0.02 ; p=.002)$ and reported an unhealthier lifestyle $(B=-0.12 ; p=.02)$ than those who completed at least one questionnaire.

After imputing missing values, total cost data was available for 1,662 of 1,733 respondents (95.90\%), and effect data was available for all 1,733 respondents (100\%) for the LFS and for 1,690 of 1,733 respondents (97.52\%) for QALYs. 
Table 8.1 Comparability of the three study groups regarding demographics, health status, lifestyle behaviour and health care and travel costs over the last three months before baseline $(N=1,733)$

\begin{tabular}{|c|c|c|c|c|c|c|}
\hline Variable & $\begin{array}{r}\text { SeqC } \\
(n=552)\end{array}$ & $\begin{array}{r}\operatorname{Sim} C \\
(n=517)\end{array}$ & $\begin{array}{r}C C \\
(n=664)\end{array}$ & $F(d f)$ & $\chi^{2}(\mathrm{df})$ & $\bar{p}$ \\
\hline \multirow[t]{2}{*}{ Age } & 47.31 & 48.15 & 48.88 & 2.61 & & .07 \\
\hline & $(S D=11.62)$ & $(S D=11.96)$ & $(S D=12.19)$ & $(2,1,730)$ & & \\
\hline \multicolumn{7}{|l|}{ Gender } \\
\hline Men & $279(50.5 \%)$ & $264(51.1 \%)$ & $366(55.1 \%)$ & & & \\
\hline Women & 273 (49.5\%) & $253(48.9 \%)$ & $298(44.9 \%)$ & & $3.1(2)$ & .21 \\
\hline \multicolumn{7}{|l|}{ Educational level } \\
\hline Low & $53(9.9 \%)$ & $64(12.9 \%)$ & $90(13.7 \%)$ & & & \\
\hline Medium & $274(51.3 \%)$ & $222(44.7 \%)$ & $319(48.7 \%)$ & & & \\
\hline High & 207 (38.8\%) & $211(42.5 \%)$ & $246(37.6 \%)$ & & $7.9(4)$ & .10 \\
\hline \multicolumn{7}{|l|}{ Income per month ${ }^{a}$} \\
\hline$<€ 1,750$ & $123(22.9 \%)$ & $123(24.7 \%)$ & $161(24.5 \%)$ & & & \\
\hline$€ 1,750-€ 3,050$ & 265 (49.3\%) & $249(50.0 \%)$ & $341(52.0 \%)$ & & & \\
\hline$>€ 3,050$ & $150(27.9 \%)$ & $126(25.3 \%)$ & $154(23.5 \%)$ & & $3.2(4)$ & .52 \\
\hline \multicolumn{7}{|l|}{ Employment situation } \\
\hline Job (paid) & 389 (72.2\%) & $350(70.3 \%)$ & $443(67.4 \%)$ & & & \\
\hline No job & $150(27.8 \%)$ & $148(29.7 \%)$ & $214(32.6 \%)$ & & $3.2(2)$ & .20 \\
\hline \multicolumn{7}{|l|}{ Marital status } \\
\hline Relationship & 423 (78.9\%) & $402(80.2 \%)$ & 507 (77.4\%) & & & \\
\hline Single & $113(21.1 \%)$ & 99 (19.8\%) & $148(22.6 \%)$ & & $1.4(2)$ & .50 \\
\hline \multirow[t]{2}{*}{ \# persons in household } & 2.83 & 2.69 & 2.71 & 1.54 & & .21 \\
\hline & $(S D=1.65)$ & $(\mathrm{SD}=1.24)$ & $(\mathrm{SD}=1.37)$ & $(2,1,695)$ & & \\
\hline \multicolumn{7}{|l|}{ Native country } \\
\hline The Netherlands & 511 (95.0\%) & $480(96.0 \%)$ & $627(95.3 \%)$ & & & \\
\hline Other & $27(5.0 \%)$ & $20(4.0 \%)$ & $31(4.7 \%)$ & & $0.6(2)$ & .73 \\
\hline \multirow[t]{2}{*}{ BMI } & 25.70 & 25.38 & 25.80 & 1.56 & & .21 \\
\hline & $(\mathrm{SD}=4.18)$ & $(S D=3.91)$ & $(S D=5.71)$ & $(2,1,714)$ & & \\
\hline \multirow[t]{2}{*}{ Psychological distress } & 44.46 & 45.00 & 44.74 & 1.00 & & .37 \\
\hline & $(S D=6.40)$ & $(S D=5.84)$ & $(S D=6.10)$ & $(2,1,687)$ & & \\
\hline \multicolumn{7}{|l|}{ Diseases } \\
\hline Diabetes & $25(4.7 \%)$ & $20(4.0 \%)$ & $23(3.5 \%)$ & & $1.0(2)$ & .61 \\
\hline Brain haemorrhage, TIA & $2(0.4 \%)$ & $4(0.8 \%)$ & $7(1.1 \%)$ & & $1.9(2)$ & .38 \\
\hline Heart attack & $8(1.5 \%)$ & $5(1.0 \%)$ & $5(0.8 \%)$ & & $1.5(2)$ & .48 \\
\hline Other serious heart disease & $4(0.7 \%)$ & $12(2.4 \%)$ & $15(2.3 \%)$ & & $5.2(2)$ & .07 \\
\hline Cancer & $12(2.2 \%)$ & $7(1.4 \%)$ & $11(1.7 \%)$ & & $1.1(2)$ & .58 \\
\hline High blood pressure & $67(12.5 \%)$ & $67(13.4 \%)$ & $112(17.2 \%)$ & & $6.0(2)$ & .05 \\
\hline Asthma, COPD & $36(6.7 \%)$ & $34(6.8 \%)$ & $43(6.6 \%)$ & & $0.01(2)$ & .99 \\
\hline \multirow[t]{2}{*}{ Lifestyle factor } & 3.26 & 3.32 & 3.27 & 0.52 & & .59 \\
\hline & $(S D=1.04)$ & $(S D=1.07)$ & $(\mathrm{SD}=1.06)$ & $(2,1,730)$ & & \\
\hline \multirow[t]{2}{*}{ Euroqol/utility (EQ-5D-3L) } & 0.89 & 0.89 & 0.90 & 0.40 & & .67 \\
\hline & $(S D=0.17)$ & $(S D=0.15)$ & $(S D=0.15)$ & $(2,1,617)$ & & \\
\hline \multicolumn{7}{|l|}{ Health care costs $(€)^{b}$} \\
\hline \multirow[t]{2}{*}{ Medication use } & 44.62 & 39.14 & 31.68 & 0.39 & & .68 \\
\hline & $(S D=256.77)$ & $(S D=313.81)$ & $(S D=204.78)$ & $(2,1,725)$ & & \\
\hline \multirow[t]{2}{*}{ Health care service } & 119.12 & 114.42 & 99.16 & 0.88 & & .42 \\
\hline & $(S D=401.90)$ & $(S D=201.50)$ & $(S D=184.52)$ & $(2,1,729)$ & & \\
\hline \multirow[t]{2}{*}{ Admissions } & 32.95 & 31.98 & 62.85 & 1.88 & & .15 \\
\hline & $(S D=231.26)$ & $(\mathrm{SD}=222.70)$ & $(S D=422.57)$ & $(2,1,728)$ & & \\
\hline \multirow[t]{2}{*}{ Other care } & 34.04 & 21.28 & 40.41 & 1.08 & & .34 \\
\hline & $(S D=220.28)$ & (SD - 157.88) & $(S D=265.29)$ & $(2,1,728)$ & & \\
\hline \multirow[t]{2}{*}{ Absenteeism from work } & 676.68 & 405.10 & 328.19 & 3.63 & & .03 \\
\hline & $(S D=3,135.31)$ & $(S D=2,076.48)$ & $(S D=1,413.07)$ & $(2,1,674)$ & & \\
\hline Travel costs & 3.20 & 3.46 & 2.97 & 0.54 & & .59 \\
\hline & $(\mathrm{SD}=10.42)$ & $(\mathrm{SD}=6.41)$ & $(S D=5.63)$ & $(2,1,579)$ & & \\
\hline
\end{tabular}

Note: ${ }^{a}$ Respondents who did not want to report their income $(n=257)$ were classified in the category " $€ 1,750$ - $€ 3,050$ ";

${ }^{b}$ Costs prior three months in euro's. 


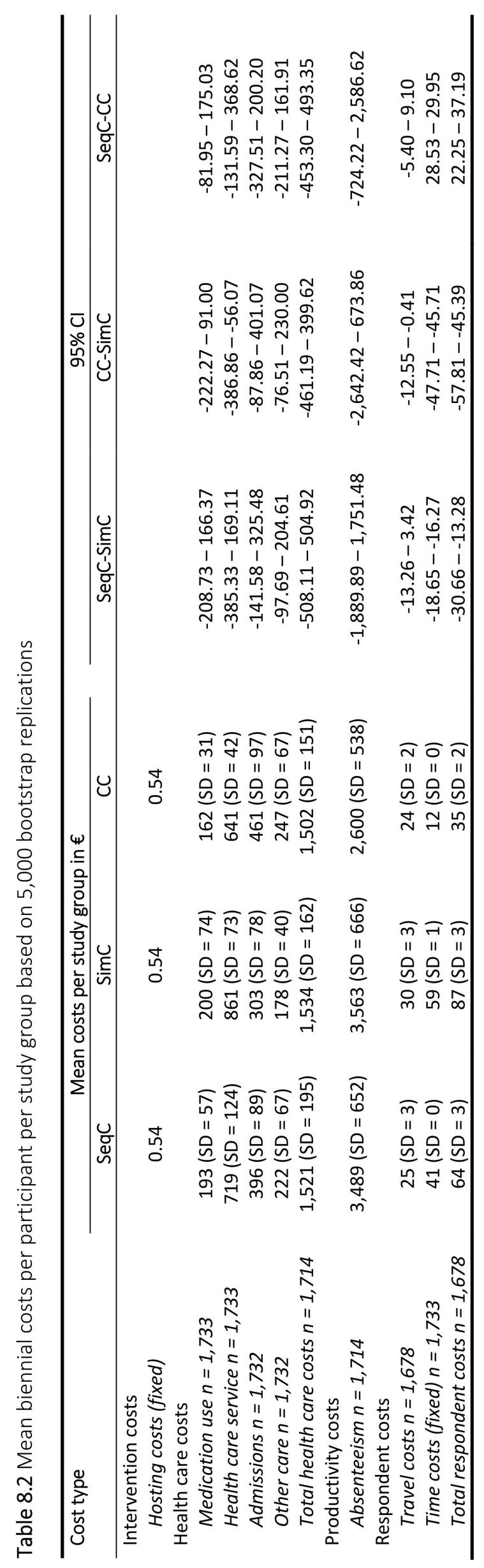


Table 8.3 Mean biennial effect on the LFS and QALY per participant per study group

\begin{tabular}{|c|c|c|c|c|c|}
\hline \multirow[t]{2}{*}{ Effect } & \multicolumn{3}{|c|}{ Mean per study group } & \multirow[t]{2}{*}{$F(d f)$} & \multirow[t]{2}{*}{$p$} \\
\hline & SeqC & SimC & CC & & \\
\hline $\mathrm{LFS}^{\mathrm{a}} n=1,733$ & $3.37(\mathrm{SD}=1.08)$ & $3.42(\mathrm{SD}=1.06)$ & $3.34(\mathrm{SD}=1.05)$ & $0.92(2,1,730)$ & .40 \\
\hline QALY (EQ-5D-3L) $n=1,690$ & $1.80(\mathrm{SD}=0.30)$ & $1.78(S D=0.31)$ & $1.82(S D=0.27)$ & $1.79(2,1,687)$ & .17 \\
\hline
\end{tabular}

Note: ${ }^{a}$ This factor could range from 0 (adherence to all guidelines) to 5 (adherence to no guideline at all), plus discounting effect.

\section{Costs and effects}

Table 8.2 shows that the simultaneous condition reported statistically significantly higher costs for health care services during the two-year period than the control condition did. However, no differences were found regarding the total biennial health care costs. The travel costs were also statistically significantly higher for the simultaneous condition than the control condition. Because the simultaneous condition was the most time-intensive condition, followed by the sequential condition, and then the control condition, total respondent costs were also higher among the simultaneous condition compared to the sequential and control conditions, and higher among the sequential condition compared with the control condition. Table 8.3 demonstrates that there were no differences found between the three study groups regarding effects on the LFS or on QALYs. Detailed information regarding the lifestyle scores at the different time points and effects of the intervention on the five behaviours are presented elsewhere (Schulz et al., 2014).

\section{Cost-effectiveness analyses}

Table 8.4 shows that the costs in the control condition were less than the costs in the sequential and the simultaneous conditions, but that the effects in the two experimental conditions were higher. From a WTP of $€ 4,594$ or more, the sequential condition appeared more likely to be cost-effective than the control condition.From a WTP of $€ 10,850$ or more, the simultaneous condition seemed more likely to be cost-effective than the control condition. When comparing the cost-effectiveness of the two experimental conditions, the simultaneous condition seemed more likely to be cost-effective than the sequential condition, from a WTP of $€ 17,106$.

The probability that the sequential condition was cost-effective at a WTP of $€ 0$ per gain in lifestyle score was $42 \%$. For the simultaneous condition, the probability was $10 \%$; and for the control condition, the probability was $48 \%$ (see Table 8.5 and Figure 8.3 ). With a WTP of $€ 18,000$ per gain in lifestyle score, the simultaneous intervention would probably (i.e. $45 \%$ ) be the most cost-effective, followed by the sequential intervention (i.e. 39\%). The two sensitivity analyses performed with different discounts confirmed this finding. Results based on the other sensitivity analyses were slightly different. The sensitivity analysis using a $\mathrm{LFCl}$ as outcome variable (i.e. correcting for the baseline lifestyle score) showed that the sequential condition might be most likely to be most cost-effective, independent of the WTP. According to the sensitivity analysis performed from a health care 
perspective, the sensitivity analysis from which costs because of work absenteeism were excluded and the sensitivity analysis from which respondents with extremely high costs were excluded, both experimental conditions were found to be more likely to be cost-effective than the control condition regardless of the WTP. Based on the sensitivity analysis with inclusion of respondents who filled out the follow-up questionnaires at least four times (50\%), the sequential condition was shown to be less likely to be cost-effective than the simultaneous and control condition for a WTP up to $€ 9,000$.

\section{Cost-utility analyses}

With regard to cost-utility, Table 8.4 shows that both the sequential and the simultaneous condition were dominated by the control condition. This means that the costs of the control condition were lower and the effects with regard to QALYs gained were higher in this group. When comparing the sequential condition with the simultaneous condition, costs were lower and effects on QALYs were larger in the sequential condition. Thus, the simultaneous condition was dominated by the sequential condition.

The probability that the sequential condition was most efficient at a WTP of €O per QALY gained was $39 \%$. For the simultaneous condition, the probability was $12 \%$; and for the control condition, the probability was $49 \%$ (see Table 8.5 and Figure 8.4). With a WTP of $€ 18,000$ per QALY gained, the costutility analysis showed that the control condition would probably (i.e. 76\%) be the most efficient condition. Indeed, Figure 8.4 shows that the control condition was most likely to be efficient, irrespective of the WTP. All but three sensitivity analyses confirmed these results. The sensitivity analysis in which costs due to work absenteeism were excluded and the sensitivity analysis in which respondents with extremely high costs were excluded showed that with a WTP up to $€ 2,980$ and $€ 7,900$ respectively, the sequential condition would probably be the most efficient (i.e. $>45 \%$ and $>48 \%$ chance, respectively). The sensitivity analysis that included only those respondents who filled out the follow-up questionnaires at least four times (50\%) showed that the simultaneous condition was likely to be more efficient than the sequential condition, independent of the WTP. In all other analyses, this was the other way around.

Table 8.4 Incremental costs and effects per gain in LFS and per QALY gained for the three study groups with a willingness to pay threshold of $€ 18,000$ for the sequential, simultaneous and control conditions

\begin{tabular}{|c|c|c|c|}
\hline Intervention & Incremental costs $(€)^{a}$ & Incremental effect ${ }^{b}$ & $\begin{array}{r}\text { Incremental costs } \\
\text { per LFS/QALY }(€)\end{array}$ \\
\hline \multicolumn{4}{|l|}{ Lifestyle factor } \\
\hline SeqC vs. CC & 183.76 & .04 & $4,594.00$ \\
\hline $\operatorname{Sim} C$ vs. CC & 868.00 & .08 & $10,850.00$ \\
\hline SeqC vs. SimC & -684.24 & -.04 & $17,106.00$ \\
\hline \multicolumn{4}{|l|}{ QALY (EQ-5D-3L) } \\
\hline SeqC vs. CC & 183.76 & -.01 & dominated $\mathrm{c}$ \\
\hline $\operatorname{Sim} C$ vs. CC & 868.00 & -.03 & dominated $^{c}$ \\
\hline SeqC vs. SimC & -684.24 & .02 & dominated $\mathrm{c}$ \\
\hline
\end{tabular}


Table 8.5 Results from cost-effectiveness and cost-utility analyses based on 1,000 bootstrap replications

\begin{tabular}{|c|c|c|c|c|c|c|c|c|c|c|c|c|}
\hline \multirow[t]{3}{*}{ Type of analysis } & \multirow{2}{*}{\multicolumn{3}{|c|}{ Study group (n) }} & \multicolumn{9}{|c|}{ Probability of highest net monetary benefit (\%) } \\
\hline & & & & \multicolumn{3}{|c|}{ WTP $=€ 0$} & \multicolumn{3}{|c|}{ WTP $=€ 18,000$} & \multicolumn{3}{|c|}{ WTP $=€ 80,000$} \\
\hline & SeqC & $\operatorname{SimC}$ & $\mathrm{CC}$ & SeqC & SimC & $\mathrm{CC}$ & SeqC & $\operatorname{Sim} C$ & $\mathrm{CC}$ & SeqC & $\operatorname{Sim} C$ & $\mathrm{CC}$ \\
\hline \multicolumn{13}{|l|}{ PRIMARY ANALYSIS } \\
\hline LFS & 552 & 517 & 664 & 42 & 10 & 48 & 39 & 45 & 16 & 26 & 68 & 6 \\
\hline QALY (EQ-5D-3L) & 535 & 501 & 654 & 39 & 12 & 49 & 21 & 4 & 76 & 7 & 1 & 92 \\
\hline \multicolumn{13}{|c|}{ SENSITIVITY ANALYSES } \\
\hline \multicolumn{13}{|l|}{ LFS } \\
\hline $\begin{array}{l}\text { Only health care } \\
\text { costs included }\end{array}$ & 552 & 517 & 664 & 38 & 27 & 35 & 24 & 70 & 6 & 24 & 71 & 6 \\
\hline $\begin{array}{l}\text { Productivity costs } \\
\text { excluded }\end{array}$ & 552 & 517 & 664 & 59 & 20 & 21 & 22 & 71 & 7 & 20 & 73 & 7 \\
\hline $\begin{array}{l}\geq 50 \% \text { of follow } \\
\text { up questionnaires } \\
\text { completed }\end{array}$ & 100 & 104 & 195 & 3 & 29 & 68 & 14 & 83 & 2 & 20 & 80 & 0 \\
\hline $\begin{array}{l}\text { LFCl to correct for } \\
\text { baseline scores }\end{array}$ & 552 & 517 & 664 & 45 & 11 & 45 & 65 & 15 & 20 & 63 & 27 & 10 \\
\hline $\begin{array}{l}\text { Respondents with } \\
\text { outliers on total } \\
\text { costs excluded a }\end{array}$ & 525 & 488 & 637 & 81 & 11 & 8 & 25 & 69 & 6 & 22 & 72 & 6 \\
\hline $\begin{array}{l}\text { Without } \\
\text { discounting costs } \\
\text { and effects }\end{array}$ & 552 & 517 & 664 & 41 & 11 & 48 & 37 & 45 & 18 & 26 & 66 & 8 \\
\hline $\begin{array}{l}\text { Discounting costs } \\
\text { and effects } 2^{\text {nd }} \\
\text { year with } 4.0 \%\end{array}$ & 552 & 517 & 664 & 43 & 11 & 46 & 39 & 45 & 16 & 27 & 66 & 7 \\
\hline \multicolumn{13}{|l|}{ SENSITIVITY ANALYSES } \\
\hline QALY (EQ-5D-3L) & & & & & & & & & & & & \\
\hline $\begin{array}{l}\text { Only health care } \\
\text { costs included }\end{array}$ & 535 & 501 & 654 & 31 & 32 & 38 & 10 & 3 & 87 & 7 & 1 & 92 \\
\hline $\begin{array}{l}\text { Productivity costs } \\
\text { excluded }\end{array}$ & 535 & 501 & 654 & 56 & 19 & 25 & 14 & 2 & 84 & 7 & 1 & 93 \\
\hline $\begin{array}{l}\geq 50 \% \text { of follow } \\
\text { up questionnaires } \\
\text { completed }\end{array}$ & 100 & 104 & 195 & 6 & 25 & 70 & 5 & 21 & 74 & 7 & 21 & 73 \\
\hline $\begin{array}{l}\text { QALY corrected } \\
\text { for baseline as } \\
\text { outcome variable }\end{array}$ & 513 & 482 & 621 & 38 & 18 & 43 & 36 & 13 & 51 & 29 & 8 & 63 \\
\hline $\begin{array}{l}\text { Respondents with } \\
\text { outliers on total } \\
\text { costs excluded }{ }^{a}\end{array}$ & 509 & 475 & 627 & 80 & 9 & 11 & 25 & 3 & 73 & 13 & 2 & 85 \\
\hline $\begin{array}{l}\text { Without } \\
\text { discounting costs } \\
\text { and effects }\end{array}$ & 535 & 501 & 654 & 38 & 12 & 50 & 22 & 4 & 75 & 8 & 1 & 91 \\
\hline $\begin{array}{l}\text { Discounting costs } \\
\text { and effects } 2^{\text {nd }} \\
\text { year with } 4.0 \%\end{array}$ & 535 & 501 & 654 & 40 & 12 & 48 & 22 & 4 & 75 & 7 & 1 & 92 \\
\hline
\end{tabular}



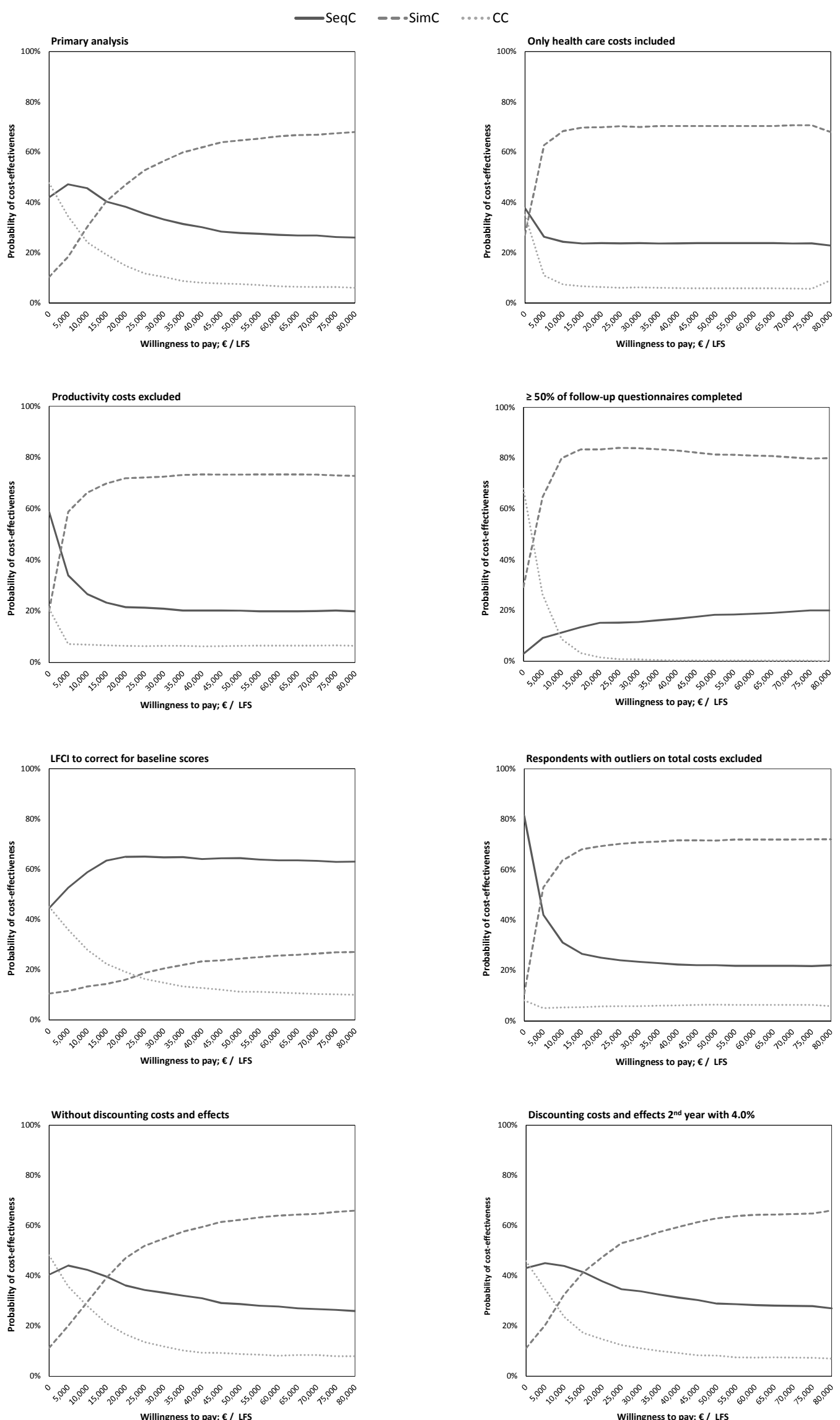

Figure 8.3 Cost-effectiveness acceptability curves for the three study groups based on primary and sensitivity analyses 


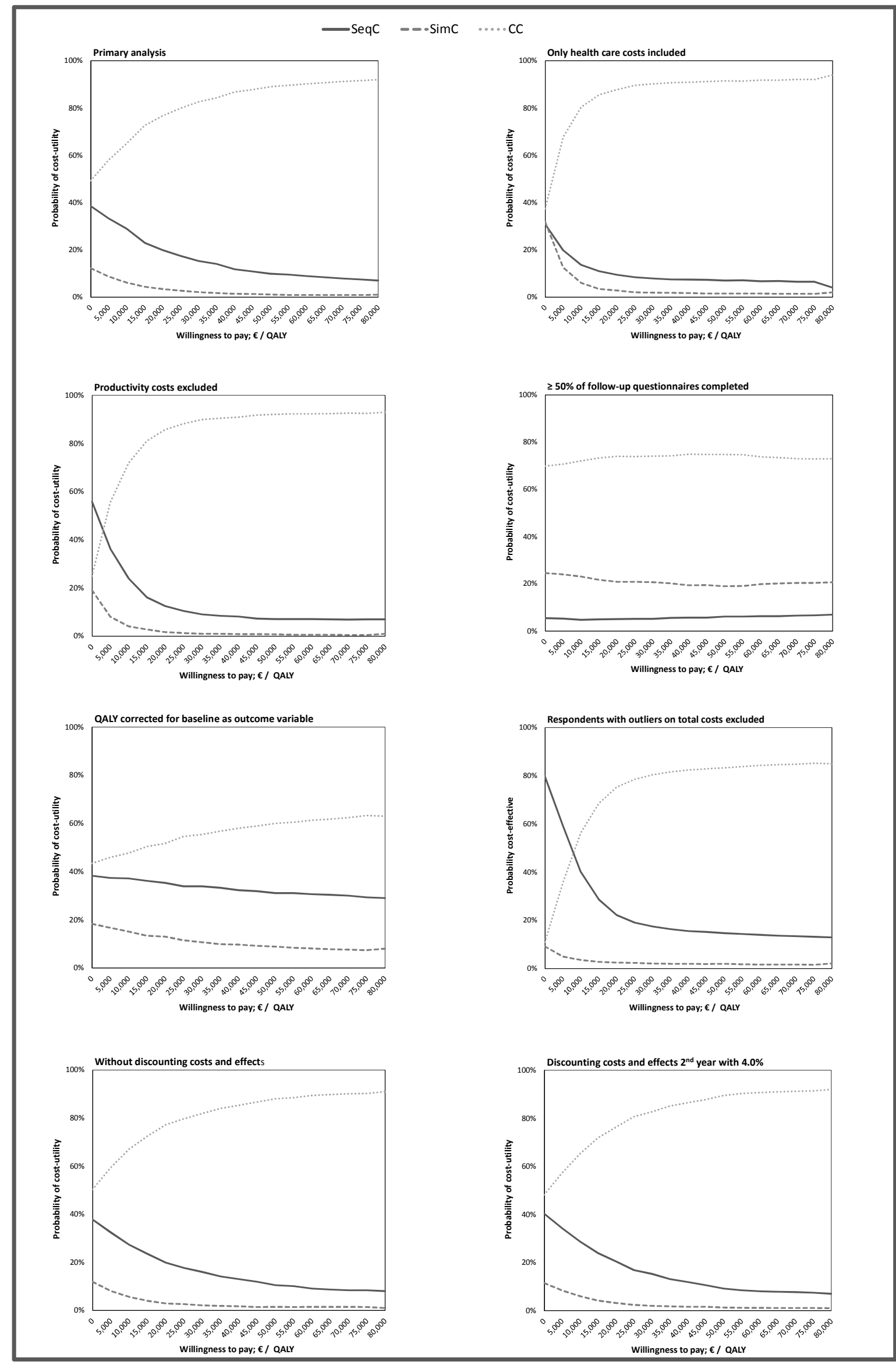

Figure 8.4 Cost-utility acceptability curves for the three study groups based on primary and sensitivity analyses 


\section{DISCUSSION}

\section{Principal findings}

An economic evaluation of two different versions of a web-based computer-tailored multiple lifestyle intervention was performed. Despite some variations in the different sensitivity analyses outcomes, the results of this study give an indication that the two tailored intervention programmes are likely to be more cost-effective when looking at lifestyles as a primary outcome, than that of a control group, in which respondents received a short tailored overview. In general, the simultaneous intervention was likely to be most cost-effective, followed by the sequential intervention. However, the results were sensitive to baseline scores. When correcting for lifestyle behaviour at baseline, the sequential intervention was probably most cost-effective. Regarding cost-utilities, the intervention received by the control group might be most preferable when compared to both lifestyle interventions (sequential and simultaneous).

With incremental costs of $€ 4,594$ per gain in lifestyle score by meeting additional public health guidelines, the sequential condition is most likely to be cost-effective. With incremental costs of $€ 17,106$ or higher, the simultaneous version of the web-based intervention is even more likely to be cost-effective than the sequential version. The incremental costs of our intervention seemed to be less favourable than the costs of $€ 160$ per guideline met in the study by Van Keulen et al. (2010a). However, the studies are hard to compare, because the intervention tested by Van Keulen and colleagues (2010a) only considered three lifestyle behaviours (i.e. physical activity, fruit intake and vegetable intake), and tailored print communication was used instead of web-based communication. Their study population consisted of older adults (aged 45-70 years). Most importantly, their control group received no intervention at all whereas in our study the control group received a minimal intervention. Information regarding one's lifestyle behaviour may be sufficient to facilitate change and improve health risk behaviours (Parekh et al., 2009). Although the effects might be modest, in the present study the difference in effects between the control group who received the minimal intervention and the experimental conditions who received the sequential and simultaneous versions of the web-based interventions might have become smaller. In a single behaviour change intervention aimed at smoking cessation, Smit et al. (2013) reported incremental costs of €5,100 per abstinent participant. This amount is comparable to our findings regarding gains in lifestyle behaviours, including smoking cessation. However, both studies (Smit et al., 2013; Van Keulen et al., 2010a) used shorter study periods (1.5 years and 1 year, respectively). This means that the cost-effectiveness regarding outcomes measured at the last follow-up in this study, which was after two years, cannot truly be compared with the cost-effectiveness results found in those studies.

Our finding that the control group might be most preferable regarding cost-utilities is comparable to the results reported by Smit et al. (2013) who found that the usual care their control group received was probably the most preferable intervention when compared with a web-based computer-tailoring intervention and a combination of the web-based intervention and face-to-face counselling by a practice nurse. A likely explanation for this finding may be that the follow-up period of two years was 
too short to find effects on quality of life. Although the intervention leads to lifestyle improvements that may prevent or postpone the incidence of a variety of lifestyle-related diseases, health gains from prevention programmes often only become noticeable many years after the costs are made (Brouwer \& Rutten, 2005). Moreover, baseline scores on the EQ-5D-3L were already high at the beginning of the study (mean $=0.90 ; S D=0.15$ ), which might be related to one restriction of this measurement tool (i.e. the ceiling effect: the tool does not differentiate between high scores of the healthy utility range) (Kopec \& Willison, 2003). Consequently, the finding that our study population consisted of people reporting high scores on the EQ-5D-3L may be an explanation for not finding any statistically significant differences in QALYs gained between the groups. For public health interventions, it might be better to use other outcomes related to quality of life (e.g. non-health outcomes, such as empowerment or satisfaction), which are more sensitive to changes in the short term because these quality of life measures tend to underestimate the relative benefits of this kind of intervention (Lorgelly et al., 2010). However, two cost-effectiveness acceptability curves (sensitivity analyses in which productivity costs were excluded, and in which respondents with extremely high total costs were excluded) showed that the sequential condition might be preferable up to a WTP of $€ 2,700$ and $€ 8,600$ respectively, which is opposite to the findings by Van Keulen et al. (2010a), whose control group was probably the most cost-effective intervention for ratios lower than $€ 2,851$ per QALY gained.

In the literature, a WTP of $€ 18,000$ is the accepted cut-off point per QALY gained (Council for Public Health and Care, 2006). However, there is no such cut-off point regarding lifestyle changes as used in our study. This makes the interpretation of the results regarding cost-effectiveness complicated. Lifestyle interventions usually aim at preventing different kinds of diseases with different burdens. This makes it difficult to determine such a cut-off point, something that has also been pointed out for increases in smoking abstinence rates (Smit et al., 2013) or for each kg of body weight lost (Van Wier et al., 2012), for example. As suggested by Tate et al. (2009), for future research, it would be good to transform the unit changes of different outcome measures into metrics that can be compared across different kinds of interventions (regardless of the target behaviour). Thus, future research should aim to define a WTP cut-off point for different lifestyle behaviours or metrics that can be used for different lifestyle behaviours.

Furthermore, there has been discussion about the rates of discounting effects (e.g. Bonneaux \& Birnie, 2001; Claxton et al., 2011; Hakkaart-van Roijen et al., 2010), especially in the field of prevention. Effect outcomes should be discounted because the value of QALYs or health increases with time (Gravelle et al., 2007; Gravelle \& Smith, 2001) and this value change is not taken into account in economic evaluations (Brouwer et al., 2005). It has been argued that the same value of discounting should be used for costs and effects to be consistent (Weinstein \& Stason, 1977), whereas it has been recommended that effects should be discounted at lower rates to correct for the increasing monetary value of health over time (Brouwer \& Rutten, 2005; Hakkaart-van Roijen et al., 2010; Health Care Insurance Board, 2006). In our study, we reported the results without discounting, with $1.5 \%$ discounting and with $4.0 \%$ discounting, based on the guidelines for pharmacoeconomic research (Health Care Insurance Board, 2006). The similarity of the findings provides evidence for the robustness of our results. 
The results revealed that respondents in the simultaneous condition reported higher costs because of health care service use during the two-year study period than did respondents in the control condition. The travel costs in this group were also higher. These costs might be related to the number of visits to care givers. The advice may have served as a kind of prompt among the respondents to ask care givers for help in improving their lifestyle, e.g. for smoking cessation guidance (Smit et al., 2013). However, it remains unclear why these costs were higher among respondents in the simultaneous intervention. It is conceivable that the marginal differences between the groups occurred because of measurement errors.

\section{Strengths and limitations}

To our knowledge, this was the first study assessing cost-effectiveness and cost-utility of two versions of a web-based tailored lifestyle intervention aiming at multiple behaviour change. Two outcome measures (i.e. a LFS and QALYS) were used to evaluate costs and intervention effects over a relatively long period of two years. Seven different sensitivity analyses, based on different views of economic evaluations found in the literature (Bonneaux \& Birnie, 2001; Hakkaart-van Roijen et al., 2010; Manca et al., 2005; Weinstein \& Stason, 1977), were performed to test the robustness of the results. This is a further strong point of this study, although some analyses showed slightly different results.

There are some limitations that should be kept in mind when interpreting the results. First, we compared our intervention groups to a control group that also received a small amount of tailored information (i.e. a personalized HRA). Because this HRA was integrated in the study website, the intervention costs were the same for all study groups. This strategy may have inflated the results, and it may be that cost-effectiveness would have been better if a different control group - who received either general information or no information at all - was used. Second, although we used a large sample $(N=1,733)$, the study suffered from high dropout rates - a common phenomenon in webbased intervention studies (e.g. Crutzen, 2009; Eysenbach, 2005; Glasgow et al., 2007); therefore, many missing values for the follow-up assessments had to be imputed. Consequently, the imputation procedure may have distorted the reliability of the findings to some extent. That is, when using the conservative LOCF-method to fill in the missing data for a large part of the sample, finding any intervention effects becomes more unlikely. The high dropout rates might have been caused by the need to assess health care costs and quality of life on a relatively large number of occasions. Although it might be good to measure health care use, medication use, absenteeism from work and quality of life every three months to counteract recall bias (Davis et al., 2013), this may also be (too) timeconsuming for some participants. This may have resulted in most of the respondents not completing all questionnaires and others dropping out of the intervention. Thus, future studies should aim to prevent loss to follow-up, by sending tailored reminder e-mails (Schneider et al., 2012), for example.

As presented in the dropout analysis, selective dropout occurred. Sensitivity analyses were performed to provide a more complete picture of the results among this selective group. Despite randomization of respondents to one of the three study groups, statistically significant differences were found with 
regard to productivity costs, and some differences almost reached statistical significance (i.e. age, high blood pressure and serious heart diseases). These differences may have influenced the results. Also, we assessed absenteeism from work, but not "presenteeism" (Hemp, 2004). Finally, we used selfreported questionnaires that are subject to bias. Additional objective measures, such as medication registration at pharmacies and data from insurance companies, and cost-diaries (Goossens et al., 2000) could be included in future studies.

\section{Conclusions}

Computer-tailored advice on lifestyle behaviours was likely to be cost-effective after 24 months, when looking at lifestyles as a primary outcome. The web-based tailored lifestyle intervention using a simultaneous approach is promising as the most cost-effective intervention in improving someone's lifestyle, followed by the intervention using a sequential approach. However, with regard to improving quality of life, the control condition seemed to be preferable. Future studies should aim to compare different computer-tailoring conditions to a control group that does not receive any personalized advice, and to identify a cut-off point for the WTP for lifestyle changes. 



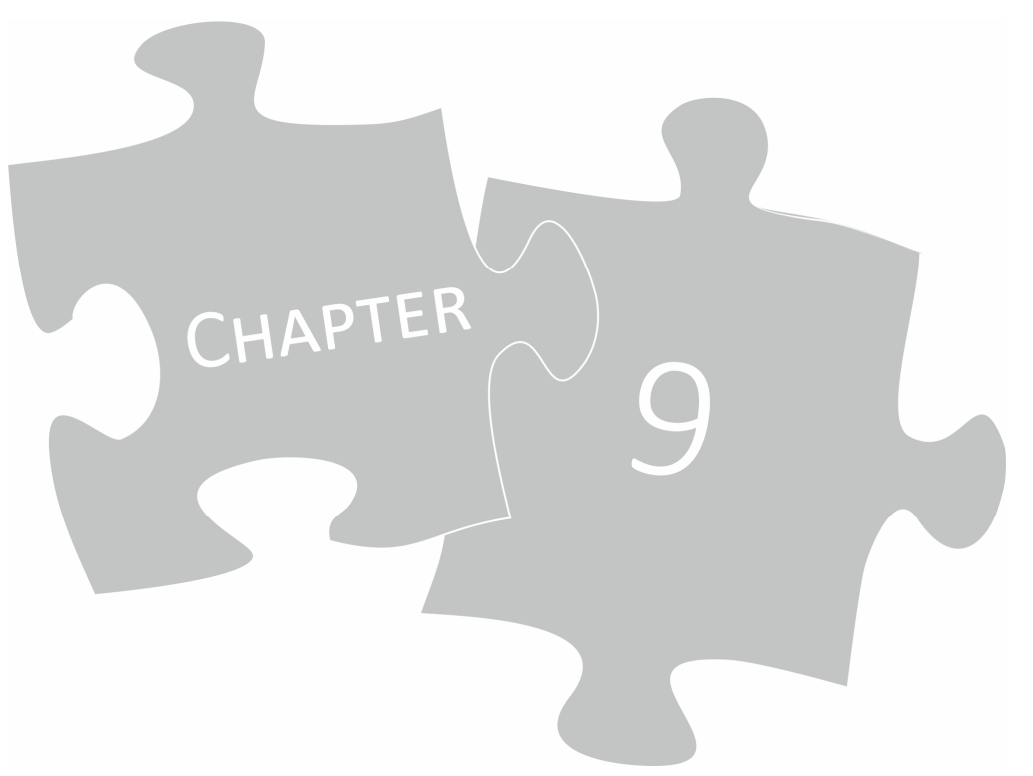

GENERAL DISCUSSION 

The main aim of this dissertation was to develop and evaluate a web-based tailored multiple behaviour change intervention with regard to its effectiveness, its cost-effectiveness and cost-utility and its use and feasibility. It is a cognitive behavioural intervention, called myHealthyBehaviour (Dutch: "mijnGezondGedrag"), aimed at inviting adults of the general population to look at their lifestyle behaviours and motivating them to change unhealthy behaviours. The intervention was disseminated via different Regional Health Authorities (RHAs) in the provinces of North-Brabant and Zeeland. Moreover, this dissertation studied the use of different tailoring strategies: (1) sequential versus simultaneous; and (2) alternating versus summative. The first type of tailoring strategy was tested within the intervention myHealthyBehaviour in a three-armed randomized controlled trial (RCT) with a follow-up period of 24 months; for the comparison between the latter types of tailoring strategies, we separately tested the alcohol module, called Alcohol - Everything within the Limits?! (German: "Alkohol - Alles im grünen Bereich"), among a sample in Germany within an RCT, with a follow-up measurement after six months.

In this final chapter, the main findings of the different studies are summarized and strengths of the dissertation are outlined. Methodological and practical limitations and implications are presented. Next, implications for theory, future research and practice are discussed. Finally, this chapter ends with some general conclusions.

\section{MAIN FINDINGS}

Our results indicate that a multiple behaviour change intervention can be a useful tool to stimulate the adoption of a healthy lifestyle. Dissemination via different RHAs, by integrating their Adult Health Monitor into the tailored lifestyle intervention, has been shown to be a feasible method to reach a great part of the general population. Reach of at-risk individuals, e.g. those with low socioeconomic status and/or an unhealthy lifestyle, was modest since those with a relatively healthier lifestyle and the highly educated were more likely to participate in our intervention. Use of the intervention, however, was suboptimal. Baseline completion rates were quite low in all study groups (around $23 \%$ to $35 \%)$. Discontinuation was predicted by a lower age and an unhealthier lifestyle and, in the sequential intervention, also by being male. Dropout rates were higher in the simultaneous condition than in the sequential condition, which might be explained by the amount of time required and information overload. The preference study, in which we investigated users' preference for certain health behaviour modules of our intervention programme, showed that the health preventive behaviour modules, especially the physical activity module, were the most popular. In spite of high dropout rates, the interventions have proven to be effective and cost-effective with regard to lifestyle improvement. In the short term (after one year), the sequential intervention may be the preferred method; in the longer term (after two years) the simultaneous intervention showed better results regarding lifestyle change. Effect sizes were small - a common finding with respect to this type of intervention (Krebs et al., 2010; Lustria et al., 2013; Webb et al., 2010). With willingness to pay (WTP) of $€ 4,594$, the sequential condition was likely to be the most cost-effective condition, whereas with 
WTP of $€ 10,850$, the simultaneous condition was likely to be the most cost-effective. The control condition, on the other hand, was probably preferred with regard to quality of life improvements. Complete case analyses revealed that personal feedback regarding drinking behaviour given in a webbased single behaviour change intervention can be an effective way to reduce alcohol intake among adults. However, this effect was not found when applying intention-to-treat analyses. In general, the intervention programme was appreciated and positively evaluated by the respondents.

\section{STRENGTHS OF THIS DISSERTATION}

The study was unique in comparing different tailoring approaches in a multiple behaviour change intervention aiming at increasing amounts of physical activity and fruit and vegetable consumption, decreasing alcohol intake and reaching smoking cessation. This range of lifestyle behaviours made it possible to have diverse outcome measures. A longitudinal design was used for a follow-up period of two years. An additional study was included in which the alcohol module was tested in a single behaviour intervention, again by comparing different tailoring strategies.

All interventions were theory-based and satisfied the five basic criteria (i.e. the 5 As) of Health Behaviour Change treatment on the Internet (HBC-I) (Evers et al., 2005): advise, assist, assess, provide anticipatory guidance and arrange follow-up. Personal advice was given regarding the respondents' behaviour and the need to change; we assessed different psychosocial determinants of the lifestyle behaviours (e.g. attitude, social influence, self-efficacy, action planning); we assisted respondents by giving relevant feedback messages and provided anticipatory guidance by focusing on the individual needs of the respondent; and we arranged follow-up sessions in which ipsative feedback was given. Therefore, reminders were sent to encourage people to revisit the websites. The I-Change model (De Vries et al., 2003) was used as theoretical framework. Earlier research indicated that extensive use of theory is associated with considerable effects in health-related behaviours (Webb et al., 2010). The programme consisted of four different steps, so that most of the I-Change concepts could receive attention in order to help people to comply with the desirable behaviour. Advantages and disadvantages of the lifestyle behaviours were discussed in relation to the attitude of the respondent. Furthermore, our interventions paid attention to the social network of each respondent and provided tailored advice on how to counteract the influence of the social environment and how to resist pressure. The interaction between the individual and his or her social environment is important in behaviour change. Both preparatory planning and coping planning were included so that respondents received feedback to enable and empower them to make the desired changes, but also to feel able to deal with possible difficult situations that might occur during and after the behaviour change process.

In this dissertation, the whole process of health promotion - from planning, to implementing, to evaluating - was applied. A wide range of studies were reported by describing reach, use (including preferences), re-use, appreciation, effectiveness and cost-effectiveness of the interventions. Besides, predictors of the various aspects were studied. 


\section{METHODOLOGICAL AND PRACTICAL CONSIDERATIONS}

The results of the different studies presented in this dissertation should be interpreted with several methodological and practical limitations in mind. Most shortcomings were already discussed in previous chapters. This section describes and summarizes the most important limitations regarding the study design, internal and external validity, dropout and its consequences, measurement instruments, outcome variables, theoretical framework, economic evaluation and (non-)exposure to the interventions.

\section{Study design}

An RCT was considered as the most appropriate design for testing the effectiveness, cost-effectiveness and appreciation of the web-based computer-tailored multiple behaviour change intervention. Our control group, however, was actually a minimal intervention group. A study by Verheijden et al. (2008) reported that people seem to be more interested in programmes that simply compare their lifestyle behaviour to relevant guidelines than in programmes that stimulate lifestyle improvement. Only a single participation would be necessary to make this comparison. This would imply that the health risk appraisal may have been sufficient to realize lifestyle behaviour change to some extent in the control group. This finding was confirmed in our study. On the other hand, more lifestyle change was found among the experimental conditions of our study and among respondents with larger exposure rates. Our results support the additional effects of tailored motivational feedback over merely behavioural feedback. In a few studies, the utilization of multiple sessions has also been shown to be more effective (Brug et al., 1998; Dijkstra et al., 1998) and may thus be recommended; however, in other studies no clear evidence of the greater effect of multiple tailoring compared to single tailoring was found and the optimal dosage is still unknown (Spittaels et al., 2007b; Velicer et al., 1999b).

Another limitation of the study design used was that respondents in the sequential condition were not granted the opportunity to receive more than two modules in total. Respondents in the simultaneous condition had the possibility to concentrate on all five behaviours, on the condition that they did not comply with the guidelines. More research is needed to test whether similar results would have been found if respondents in the sequential condition had also had the option to receive all modules.

\section{Internal and external validity}

We controlled for time effects in our evaluation regarding behavioural change (chapter 7). To increase internal validity of the study results, we controlled for potential covariates (e.g. baseline differences between study groups with regard to demographics, health status and lifestyle behaviour; predictors of dropout) in all our analyses using regression techniques (chapters 3, 4, 5, 6 and 7). This was necessary because selection bias occurred during the inclusion of respondents, as well as selective loss to follow-up. 
The external validity of the study results may be affected by selective enrolment and selective retention. People who search for health information on the web are more health-oriented (DuttaBergman, 2004). This implies that these people often live more healthily and may need those programmes less. Moreover, external validity may have been threatened by the large amount of dropout. Thus, the generalizability of our results may be limited to those people who are motivated to take part in such an intervention over a longer period of time. To increase external validity, we chose to not only include fully complete cases in the effect analyses (chapter 7), but also include T0-T1completers and T0-T2-completers in order to maximize the number of included cases. However, the studies might have suffered from selection bias. Therefore, one should be cautious when generalizing findings from this type of intervention to the general population.

The respondents for the multiple behaviour change intervention were mainly recruited by the different RHAs. The respondents for the single behaviour change intervention regarding alcohol intake were recruited by an online panel. It might be that selective groups were included in the studies and that recruitment via different channels (e.g. general practitioners (GPs) or companies) may lead to different findings. First, earlier research has shown that recruitment strategies may influence reach and retention of participants, which, in turn, may influence behaviour change. For example, Stanczyk et al. (2013) found that smokers recruited by GPs were lower educated and suffered more often from chronic obstructive pulmonary disease than those recruited by Internet. Moreover, respondents recruited by GPs more often revisited their web-based smoking cessation intervention than those recruited by other strategies (e.g. newspapers and Internet). Smit et al. (2012b) compared two recruitment strategies on reach and effectiveness of a web-based tailored smoking cessation intervention and found lower retention and lower success rates among respondents recruited via a large media campaign than among those recruited by GPs. Second, respondents in our studies could receive incentives, which may have motivated them to participate, rather than their being motivated to change health behaviours. While chances to receive these rewards were small in the multiple behaviour change intervention (we raffled $300 \times 50$ euros), all respondents in the single behaviour change intervention received bonus points (these points could be exchanged for cash, a gift voucher or a charitable donation) provided that they completed the measurement sessions.

\section{Missing values and imputation techniques}

In addition to complete case analyses, we performed intention-to-treat analyses by using different imputation techniques to correct for the high number of missing values: (1) last observation carried forward (LOCF); (2) mean imputation; and (3) multiple imputations (MI). All of these methods are related to various distinct advantages and disadvantages. In chapter 6 , we compared the results based on complete cases with two of the imputation techniques, namely LOCF and MI. The results differed significantly when applying these two imputation techniques. Currently, $\mathrm{Ml}$ is reported as the most preferred technique to handle missing data (Blankers et al., 2010); however, some criticism of MI has been mentioned in the literature: there is no consensus about the number of imputed datasets needed (Allison, 2012; Blankers et al., 2010; Bodner, 2008; Graham et al., 2007; White et al., 2011); 
the results may strongly depend on the imputation model that is created (Sinharay et al., 2011; White et al., 2011), as well as on the programme that is used (Blankers et al., 2010); the strategies for how MI should be used are not clearly documented (Allison, 2012; Bodner, 2008; White et al., 2011); and the maximum number or percentage of missing values seems to be unclear (White et al., 2011). Based on experiences with the multiple ways to deal with MI in our study, we can conclude that the use of the $\mathrm{Ml}$ technique results in unreliable estimates when the number of missing values is high. Moreover, the reliability may depend on the kind of variable that is missing and will be imputed: it is conceivable that a missing value on a variable that is measured at another time point can be more reliably imputed than, for example, a demographic variable that is only measured once. In our study, for example, the variable with regard to household income was not answered by $14 \%$ (programme: myHealthyBehaviour) and 23\% (programme: Alcohol - Everything within the Limits?!), respectively. In chapter 7, mixed model analyses were used in addition to regression analyses (without imputed data). Mixed model analyses allow for inclusion of all cases (despite missing values on the outcome variable), and are valid in the case that the missing values satisfy the missingness-at-random assumption (Enders, 2010). In our study, results differed between complete cases analyses and mixed model analyses. Therefore, we believe it is fair to assume that missing values were not at random.

\section{P-values and effect sizes}

P-values should be cautiously interpreted. The cut-off point which is used to indicate an effect is widely taken as .05; however, this is an arbitrary figure (Cohen, 1994). Statistically significant findings at, for example, a significance level of .05 can still yield false-positive (type I error) as well as falsenegative (type II error) results. False-positive results are common among large samples. This means that results may be dependent on the sample size; therefore, careful interpretation of results is warranted. On the other hand, a small effect size and even a non-significant result can have high relevance. Interventions with low effect sizes but a high reach can still have great public health impact. Sometimes the terms "borderline significance" or "marginal significance" are used when p-values lie between .05 and .10, but this is not consistently done in the literature. Borderline significant effects may occur when there is a clinically relevant effect but a large standard error, often caused by insufficient respondents (underpowered study). In any case, when a borderline effect is found, no strong evidence can be given for or against an intervention (Hackshaw \& Kirkwood, 2011). In the effect studies of this dissertation (chapters 6 and 7), both significant and borderline significant results were found with regard to the variable "condition". We added effect sizes (ES) (Cohen, 1988) to assist the reader in interpreting the findings. In chapter 7, for example, a p-value of .08 and an ES of 0.19 were found after 12 months when comparing the simultaneous condition to the control condition; after 24 months, the p-value was .048 and the ES was 0.18. Thus, although the $p$-values differed from each other with respect to the generally accepted $p$-value cut-off (i.e. $p<.05)$, the ES were similar and suggested small effects. 


\section{Power and dropout}

In the study protocol (chapter 2), we reported a required sample size of 2,826 respondents for assessing the effectiveness of our intervention when comparing three groups. In the study describing the intervention effects (chapter 7), we reported a sample size of 1,182 respondents was needed. The first power analysis (as described in chapter 2 ) was based on the assumption that only people who did not comply with the different health behaviour guidelines were included; the latter power analysis (as presented in chapter 7) was added later on, since all respondents (irrespective of their baseline lifestyle behaviour) were included in our effectiveness study. Nevertheless, we did not manage to reach the intended total number of respondents at all measurement points, which forms a limitation of the different studies. Thus, the studies were underpowered to some extent, which might have caused type II errors.

\section{Measurement instruments}

Self-reported questionnaires were used for all outcome variables, which are related to recall bias and socially desirable answers. We made no use of objective measures. To assess smoking abstinence, for example, nicotine validations could be included as an objective measure; or with regard to physical activity, accelerometers could be used to assess levels of physical activity objectively. However, these methods are more costly. In our studies, we made use of different validated questionnaires to measure the five lifestyle behaviours and psychosocial constructs related to these behaviours. Despite their validity, often systematic overestimation of, for example, the amount of physical activity and fruit and vegetable consumption appears when using self-reported measures. However, when these "errors" occurred within persons over time and were the same between the different study groups, it did not affect the results substantially.

Furthermore, the manner in which behaviour was assessed may strongly influence results. In an earlier study by Van Keulen et al. (2010b), physical activity, fruit intake and vegetable intake were measured in two different ways: with a single item and with multiple items. When calculating if respondents comply with the three guidelines, large differences were found. In our study, we included a shortened version of the physical activity questionnaire after 12 and 24 months. In this adapted version of the SQUASH, we asked respondents to indicate the number of minutes for which they were at least moderately physically active on each day of the week (from Monday to Sunday). We calculated the correlations based on the guideline status and the time during which respondents were physically active. The results showed a low correlation between outcomes based on the SQUASH and on this adapted questionnaire. Correlations were calculated between the numbers of minutes and based on meeting the Dutch physical activity guideline when using the SQUASH versus the adapted questionnaire. Both continuous and logistic outcomes differed significantly. It seems that the short version of the SQUASH may have overestimated levels of physical activity. In line with our expectation, we indeed found that the measured mean daily duration and the number of respondents meeting the guideline were much lower when using the self-developed adapted questionnaire (mean $=43.41$ 
minutes; SD $=54.33 ; 69.3 \%$ ) compared to the SQUASH (176.71 minutes; SD $=174.76 ; 89.4 \%$ ). Hence, more objective measurement methods could be integrated as control items.

A second explanation for the high compliance rates with regard to the physical activity guideline may be the fact that we did not measure the intensity of the different kinds of physical activities, since this aspect is not included in the short version of the SQUASH questionnaire that we used (National Institute for Public Health and the Environment, 2005). When using a comparable method by calculating the time of moderate or intense physical activity on the basis of the MET values, around $65 \%$ of the respondents would adhere to the guideline. This is more in line with previous research, but still fairly high (De Vries et al., 2008a).

Consequences of recall bias - resulting in underestimation or overestimation of behaviour - may be that feedback messages were not justified. In our intervention, we included many questions to allow reliable and individualized feedback (Vandelanotte et al., 2004). However, the self-report assessment may have resulted in incorrect estimates of the respondents' actual behaviour, which means it is possible that mismatched feedback and advice may have been given (Vandelanotte \& De Bourdeaudhuij, 2003).

\section{Outcome variables}

For both the effectiveness study (chapter 7) and the cost-effectiveness study (chapter 8), a risk factor score and lifestyle score, respectively, were calculated. This was done by summing up all unhealthy and healthy lifestyle behaviours (based on the public health guidelines defined for the different behaviours), respectively, as has also been done before (e.g. Jiao et al., 2009; Meng et al., 1999; Parekh et al., 2012; Spencer et al., 2005; Tamakoshi et al., 2009). On the one hand, it is good to have an overall score since lifestyle as a whole may be a better predictor of unhealthy outcomes than one single risk behaviour, but this score has three important limitations. First, nutrition is overrepresented due to the inclusion of vegetable and fruit consumption. Second, a dichotomous scoring system is used, which may have resulted in loss of study power. In this way, small changes may be undetected (Jiao et al., 2009; Parekh et al., 2012). Third, not every behaviour is equally responsible for burden of disease (Ezzati et al., 2002; Meng et al., 1999). Maybe, as also suggested by Parekh et al. (2012), the different behaviours should be weighted differently (i.e. in accordance to their relative impact on health). Smoking and alcohol are, among others, associated with the greatest burden of disease, causing, for example, lung cancer and coronary heart disease, different kinds of cancers and cirrhosis of the liver, respectively (Ockene et al., 1990; Rehm et al., 2003). Estimators of different algorithms to assess lifestyle behaviours were beyond the scope of this study, but are recommended for future studies. 


\section{Theoretical framework}

Earlier research has pointed out that effectiveness of Internet-based interventions is associated with more extensive use of theory, especially the use of the Theory of Planned Behaviour (TPB) (Webb et al., 2010). The theoretical framework used for the development of the interventions described in this dissertation was the I-Change model (De Vries et al., 2003), which contains the concepts of the TPB. However, not all concepts of the I-Change model were integrated in our interventions; for example, risk perception was left out, which might have been useful to increase awareness. Moreover, we measured different predisposing factors, but did not tailor most of them within the interventions.

In our interventions, respondents had to pass through a predefined order of steps per module (i.e. 1. attitude; 2 . social influence; 3. preparatory planning; 4. self-efficacy and coping planning). Thus, all respondents received feedback about all psychosocial constructs. Despite some criticism regarding the stage of change model - which is integrated in the I-Change model - various studies have found that certain factors may be more relevant in certain stages than others (De Vet et al., 2005; De Vet et al., 2006; Etter \& Sutton, 2002; Herzog et al., 1999; Segan et al., 2004; West, 2005). According to De Vries and Backbier (1994), people in different motivational phases differ in terms of attitude, social influence and self-efficacy. In the earlier phases, attitude seems to be relevant and should be targeted; afterwards, social influence becomes more and more important, whereas in the later phases, levels of self-efficacy should be increased. Also, the Health Action Process Approach (HAPA) (Schwarzer, 1999; Schwarzer et al., 2011) states that people in different stages need different types of information. According to this model, pre-intenders may need information regarding risk perception and outcome expectancies whereas intenders need to concentrate on planning strategies. Self-efficacy may play an important role in all motivational phases, but different kinds of self-efficacy may be relevant (i.e. task self-efficacy, maintenance self-efficacy and recovery self-efficacy). Perhaps phase-matched interventions may be useful to stimulate a healthy lifestyle by use of computer-tailored interventions (Schulz et al., 2012a).

Although use of the I-Change model as the theoretical framework in computer-tailored programmes has shown positive results regarding the improvement of different lifestyle behaviours (Elfeddali et al., 2012a; Smit et al., 2012a; Van Stralen et al., 2009a), other social cognitive models could be used, maybe in addition to the I-Change model. With regard to the low usage of our lifestyle interventions, motivation-enhancing interventions may be required. Integration of the self-determination theory, initially developed by Deci and Ryan (1985), into motivational approaches might be an option as this theory explicitly distinguishes intrinsic and extrinsic motivation. Especially by increasing intrinsic motivation, intervention use and re-use may also be increased. Another important aspect of the selfdetermination theory is the need for autonomy - one of humans' three basic needs (i.e. competence, relatedness, and autonomy; Deci \& Ryan, 2000). In a computer-tailored programme, different options and choices could be offered to participants, for example the possibility to choose which and how many lifestyle behaviours they wish to improve. Particularly with regard to the application of different tailoring strategies, this aspect may be taken into account. 
Moreover, motivational interviewing, which is defined as a "collaborative, person-centered form of guiding to elicit and strengthen motivation for change" (Miller \& Rollnick, 2009; page 137), could be integrated in computer-tailored interventions (Friederichs et al., 2013; Resnicow et al., 2008; Voogt et al., 2013). For example, motivational interviewing principles could be incorporated by using openended questions to enable autonomy support and multiple choice questions to enable skilful reflections (Friederichs et al., 2013). A study by Van Keulen et al. (2011) reported that tailored print communication, telephone motivational interviewing and a combination of the two were all successful in changing multiple behaviours. Though, in general, the effects of tailored advice were slightly better than the effects of motivational interviewing or the combination, the best strategy may depend on the target behaviour. As shown in the study by Van Keulen et al. (2011), the combination of tailored advice and motivational interviewing resulted in the highest ES with regard to physical activity change in the long term (73 weeks). With regard to effects on fruit and vegetable consumption, on the other hand, highest ES were found when using tailored feedback only.

\section{Economic evaluation}

Both the sequential and the simultaneous lifestyle intervention were likely to be cost-effective when it concerned the lifestyle factor, whereas cost-utility - taking quality of life as the outcome measure was highest for the control condition. However, there is no accepted cut-off point for the WTP per gain in lifestyle behaviours, which made it impossible to draw firm conclusions. In the literature, a WTP of $€ 18,000$ is accepted per additional QALY; however, it is questionable whether an identical amount for improvements in lifestyle (e.g. WTP per additional guideline met) is accepted. The WTP may even depend on the individual health risk behaviour, since different health risk behaviours have different impacts on morbidity and mortality (Ezzati et al., 2002).

Furthermore, the EQ-5D-3L was perhaps not the best measurement instrument for measuring quality of life. This tool does not differentiate between high scores of the healthy utility range (Kopec \& Willison, 2003), and our study group already reported high quality of life scores at baseline. Moreover, effects in the shorter term are likely to be difficult to detect. It may even be that people experience more negative feelings, for example due to encountering nicotine withdrawal and difficulty refraining from smoking or drinking (Wiggers et al., 2006).

A further limitation is that only a selective subgroup $(n=1,733)$ of the total study group (described in chapter 7) was included in the analyses of the cost-effectiveness study (chapter 8). This was done to prevent inaccurate estimations for health care, medication use and quality of life for those respondents who did not answer these questions at least once (at baseline). 


\section{Exposure and non-adherence to the interventions}

Our results showed that there was a positive relationship between behavioural change and the amount of exposure to the interventions. This finding is in line with earlier findings showing a doseresponse relationship between smoking abstinence and the number of programme elements completed by the respondents in a web-based computer-tailored smoking relapse prevention programme (Elfeddali et al., 2012a). However, we do not know which modules were actually seen and read by the respondents. Thus, this aspect could not be taken into account, but it is recommended that future research better analyses dose-response relationships. Besides, when looking at the differential effects on behaviour, we did not correct for the modules people indeed chose and completed. Stronger effects might have been found when only including those respondents who completed corresponding modules, for example. Besides, bias might have been introduced by the assumption that in some cases it was not the same person who completed the baseline and postmeasurements, although we tried to reduce this bias to a minimum. At all measurements, we included control items (i.e. gender, age and height). We excluded cases that showed inconsistent data to minimize this potential limitation.

\section{IMPLICATIONS FOR THEORY AND FUTURE RESEARCH}

It is essential to further optimize reach and use by putting additional effort into increasing interest in the lifestyle intervention, especially among at-risk individuals, and encouraging and reminding respondents to actually use the intervention. Therefore, strategies to optimize programme completion as well as continued use of computer-tailored interventions should be studied. In multiple behaviour change interventions, physical activity could be studied as a potential gateway behaviour in tailored eHealth lifestyle promotion. In the following, future directions for research are outlined.

\section{Stimulating use and re-use and decreasing dropout}

Whereas in a classical laboratory setting respondents often feel obliged to stay and finish the experiment, respondents in web-based studies can much more easily leave the session at any time (Frick et al., 2001). In our multiple behaviour change study, dropout rates were high. However, the participation rate after 24 months was higher compared to the study participation rate at 12-month follow-up as we used different strategies to increase the participation rate after 24 months: we sent two reminders in a short period; we emphasized that respondents only had to fill in a short questionnaire; we included information about the health risk appraisal - also for the control group; and we integrated the cost-effectiveness questionnaire into the last follow-up measurement so that people did not have to open two links in one e-mail, as was the case during the study period (before the 24-month follow-up measurement). These methods may have contributed to the increased participation rate at the last measurement point. 
Earlier studies reported other strategies that can be used to increase participation in a study and to prevent dropout. One technique - which we applied in our interventions - is to assess participants' personal information at the beginning of an experiment. In this way, more complete demographic data about the participants can be reached (Frick et al., 2001). Further, a lottery or other financial incentive may be included so that respondents can win or get prizes (Frick et al., 2001). Rewarding people can help to increase participation rates (Verheijden et al., 2008). Financial incentives are a technique proven to be effective in reducing dropout. We used this technique by raffling an amount of $€ 50.00300$ times.

When integrating a health behaviour change intervention in another programme (e.g. the Adult Health Monitor system), the period between participation in the two mediums should be fairly short to prevent people forgetting that they agreed to participate in the study and to ensure that the personal advice provided is still up to date. Our respondents were approached after a period of three weeks, which might have been too long. Also, invitations for re-visits could have been sent earlier (we sent these after three months). A study by Schneider et al. (2013a) found that respondents who received a reminder after a two-week period logged in to the lifestyle programme more often than respondents who got a reminder after six weeks. Thus, relatively short prompt timing has shown to be beneficial. People might get the option to choose if they want to receive reminders and how often or after which periods. It is important to motivate people to revisit the intervention programme. Yet a study by Verheijden et al. (2007) shows that re-visits to Internet interventions are uncommon: only $10 \%$ of their participants visited the programme twice or more $(7.6 \%$ twice; $1.9 \%$ three times; and $<1.0 \%$ four times). One strategy may be to indicate at the start of the intervention "that behaviour change does not occur overnight" and explain that follow-up visits are possible (Verheijden et al., 2007), or possibly needed, as behaviour change often requires multiple sessions.

Other options for increasing participation may be face-to-face contact before visiting the programme for the first time (Spittaels \& De Bourdeaudhuij, 2006). In their study, Spittaels and De Bourdeaudhuij (2006) found that more participants with initial face-to-face contact (46\%) registered on their tailored physical activity website compared to participants without personal contact (6\%).

To increase participation and prevent high dropout rates, it is recommended to make web pages shorter, more attractive and faster loading. According to the model of Internet interventions (Ritterband et al., 2009), various characteristics of a website influence its use, such as appearance (e.g. colour usage and organization), burdens (e.g. difficulty of use and length), content (e.g. accurate, clear and simple) and assessment (e.g. tailoring).

As shown in the review by Kohl et al. (2013), low use of online interventions is common across all different behavioural domains. Earlier studies demonstrated that a large amount of potential respondents of health behaviour change interventions were not motivated to change. According to the transtheoretical model, they are in the precontemplation or contemplation stage. Lack of motivation may be related to dropout. Thus, new, innovative directions are needed that increase motivation for health behaviour change among less interested people (e.g. Miller \& Rollnick, 1995). Engagement in eHealth is a precondition: users should be involved in health content in ways that 
motivate and lead to health behaviour change (Lefebvre et al., 2010). It is important that respondents feel confident and motivated in succeeding in changing. As also stated by Verheijden et al. (2007), lack of interest may be one important factor explaining the limited number of re-visits and the high dropout rates of our intervention. Respondents may also have dropped out because of technical problems, as indicated by some e-mails that we received. It is important to prevent technical errors and other problems, such as navigation difficulties, too-slow loading times and e-mails being categorized as spam. Future research is necessary to study why people drop out of web-based interventions. However, due to ethical constraints, this is not always possible.

Last but not least, brief engagement in a web-based programme, dropout and low re-visit rates may not necessarily be an indication of failure. Respondents may disengage from a programme after successful behaviour change (Postel et al., 2010; Postel et al., 2011b). For example, a successful quitter may disengage from a smoking cessation intervention so that he or she is not reminded of his or her previous tobacco use (Strecher et al., 2008). It is impossible to give a concrete conclusion since we do not know the reasons for intervention disengagement. Hence, assessment of reasons for study disengagement is desirable in order to better test effects and cost-effectiveness of lifestyle behaviour change interventions.

\section{Exploring the suitability of web-based computer-tailored interventions for risk groups}

Multiple behaviour change interventions have been recognized as a promising method to improve health, to increase efficiency of health behaviour interventions and to reduce health care costs (Glasgow et al., 2004; Orleans, 2004; Prochaska et al., 2005; Pronk et al., 2004b; United States Department of Health and Human Services, 2000). Our studies succeeded in reaching small but significant effects in lifestyle change after following a web-based computer-tailored intervention. The studies were executed among adults of the general population. Although the studies show promising results regarding lifestyle behaviour change, it might be easier to find clinically relevant effects in certain specific subgroups, such as patients with a particular disease, who often experience higher risk perceptions and the necessity to improve their lifestyle (Silagy et al., 1993). Moreover, we hypothesize that computer-tailored programmes are most applicable for people with a minor problem behaviour (e.g. drinking a little bit too much). Earlier research has indicated that web-based programmes succeeded in reaching people who are often not reached by face-to-face interventions (Kypri et al., 2005; Postel et al., 2005; Postel et al., 2011a). Hence, computer-tailored programmes may be especially suitable for people with problems, for example alcohol problems, who do not want to receive professional help, e.g. by visiting a psychologist. From studies testing mental health interventions, such as a web-based support group for gynaecologic cancer patients with psychosexual distress (Wiljer et al., 2011) or web-based counselling for problem drinking (Riper et al., 2008) and problem gambling (Rodda et al., 2013), it can be seen that anonymity and confidentiality are reasons for using these kind of interventions (Griffiths et al., 2006). Verheijden et al. (2007) found that a healthy lifestyle was related to re-visits. One notable finding was that overweight or obese participants visited the programme more often than people of normal weight. A possible explanation could be that 
obesity is a stigmatizing disease. In this case, a non-face-to-face intervention may be preferred (Verheijden et al., 2007). Another study reported that chronically ill patients are more likely to look for health information on the web compared to those without a chronic condition (Bundorf et al., 2006). More research is needed to study which groups benefit most from web-based computer-tailored interventions, for which groups this kind of intervention is sufficient to reach behavioural change and which groups need additional interventions.

\section{Discovering the most effective tailoring strategy}

In this dissertation, different tailoring strategies were tested and compared (i.e. sequential versus simultaneous; alternating versus summative). In general, both the sequential and the simultaneous strategy have been proven to be effective and cost-effective in improving lifestyle. However, no firm conclusion can be drawn about the "best" strategy and still many questions remain unanswered: Does the preference for one strategy depend on the individual's readiness to change (Koshy et al., 2012)? How many health behaviours can be targeted effectively in a single intervention without "overloading" participants (Taylor et al., 2004)? Which behaviours should be combined in one intervention to reach optimal effects? Within the sequential approach, what is the optimal interval between the intervention sessions (Vandelanotte, 2013)? Which behaviour change strategies are most optimal with respect to multiple behaviour change via web-based interventions? Which theories are most effective in computer-tailoring interventions with regard to use and effectiveness? Which determinants should be intervened on in such interventions?

As stated earlier, a mix of the sequential and the simultaneous strategy may be recommended (i.e. preference-based approach). Using this strategy, respondents can choose the behaviour modules they want to complete without any limitation to the number of modules they fill in. Alternatively, given the fact that some studies suggest the existence of various lifestyle behaviour clusters, a cluster approach could be used as well (De Vries et al., 2008a; Kremers et al., 2005). In this way, only behaviours that often co-occur are addressed (e.g. unhealthy diet and physical inactivity). However, further research is necessary to find out whether addressing clusters of related behaviours will indeed result in better effects, less demotivation and less ego depletion than a simultaneous approach. The best approach may depend on personal characteristics, current health behaviours and motivation. Further research should be carried out to identify these moderators of intervention suitability and effectiveness.

The comparison between an alternating and a summative approach showed no indication that one of the tailoring strategies was more effective in lowering alcohol intake. Programme appreciation was comparable for the two experimental groups. Nevertheless, the lower attrition rates we found during the first visit suggest that the version of the intervention with alternating questions and advice may be preferred in order to realize better public health impact, as defined by reaching $x$ effect. Additional research is needed to further study the most optimal method of feedback delivery. 


\section{Improving the handling of missing data}

Intention-to-treat analyses are regarded as the gold standard in RCTs (Moncur \& Larmer, 2009; Tillmann et al., 2001). Different imputation techniques can be used to replace missing values. Currently, multiple imputations are widely used and well accepted. However, further research is needed to determine conditions of the multiple imputation technique, including the necessary number of imputed datasets and the composition of a suitable imputation model, as well as to find the best kind of programme suitable for using this technique (e.g. SPSS, Amelia, MICE or NORM) (e.g. Blankers et al., 2010; White et al., 2011). Moreover, future research should investigate the reliability of imputed data regarding different types of variables (e.g. demographic variables that are measured only once versus lifestyle behaviour on a follow-up measurement).

\section{Enhancing measurement instruments and outcome measures}

Although the SQUASH questionnaire has proven to be fairly reliable and valid among adults (WendelVos et al., 2003), we see a need for future research on how to further increase its validity and reliability. Based on our results, we suggest that this instrument overestimates amounts of physical activity. Since this questionnaire is used by the different RHAs and other instances in the Netherlands, a good and precise instrument for assessing amounts of physical activity is of great importance. In addition, more research is needed to develop better algorithms in order to better measure lifestyle behaviour change, for example by weighting the multiple behaviours differently.

\section{Optimizing standards for cost-effectiveness analyses}

To date, there is no accepted cut-off point for the WTP for lifestyle improvement, for example per additional guideline met. Future research should define the maximum societal WTP for smoking abstinence - as already indicated by Smit et al. (2013) and Berndt (2013) - acceptable drinking limit, sufficient amount of physical activity and sufficient fruit and vegetable intake. It may be difficult to define the WTP per additional guideline met, since this amount may depend on the specific lifestyle behaviour. Moreover, the WTP may depend on current behaviour, since the change necessary to meet a certain guideline differs between people. In other words, for a small change, the WTP should be smaller than for a bigger change. Future studies should also evaluate the use of the EQ-5D-3L to calculate the QALYS. Potential other domains should be added to the questionnaire, which are sensitive to changes in the short term. As also outlined by Alayli-Goebbels (2013), it is important to develop validated instruments that allow for consideration of impacts on wider domains of well-being. Methods in data collection should also be improved by including objective measures and increasing response rates. Finally, the long-term impact of the web-based computer-tailored lifestyle interventions should be investigated, for example future health care costs, morbidity rates and years of an individual's life. Therefore, economic modelling studies in the field of public health are needed (Feenstra et al., 2005; Sculpher et al., 2006). 


\section{IMPLICATIONS FOR PRACTICE}

\section{Optimizing future lifestyle interventions}

The high dropout rates found in our studies and the findings regarding appreciation of the interventions showed that it is recommended to consider improving our web-based computer-tailored interventions regarding different domains. In this section, some proposals are made that could be considered when making attempts to optimize the programme.

First, the tailoring strategies could be optimized by letting persons decide by themselves which and how many behaviour modules they want to complete at what time. Thus, a preference-based tailoring strategy could be used, which forms a combination of the sequential and the simultaneous approaches. Alternatively, a cluster-based method could be used. Hereby, addiction behaviours (e.g. alcohol intake and tobacco use) could be integrated in one module and stimulated simultaneously, and health-promoting behaviours (e.g. physical activity and nutrition behaviours) could be combined in another module.

Second, the high incompletion rates indicate the need to make the programme more attractive and to shorten it, maybe by reducing the number of questionnaire items. As reported by Verheijden et al. (2008), a visit should not be longer than 17 minutes. On the other hand, sufficient information needs to be given to ensure effectiveness of the programme. Earlier research has shown that visitors should be carefully guided through the intervention (Crutzen et al., 2012). In line with this finding, we used a tunnelled version of the modules which means that the different intervention parts were predetermined and no parts could be skipped. However, in our interventions, this structure could be made clearer for the visitor of the website. Relating to this - and as also suggested by participants progress bars could be added to visualize the progression of the visit. Additionally, to make the advice more interesting and diversified, ipsative feedback could be added about changes in psychosocial factors.

Third, a website should be updated regularly (Leslie et al., 2005). Our websites were updated monthly. For example, we changed the vegetables of the season and the (alcohol-free) cocktail recipes. However, more changes could have been incorporated in the intervention, for example by using social media with daily updates. These adjustments may result in more re-visits.

Fourth, respondents indicated they would have liked the possibility to clarify their answers, and questions and advice should be made more personal, for example by asking about reasons for noncompliance with the guideline(s) for certain behaviours or by including advice for specific (risk) groups. We received feedback from respondents that they were, for example, not able to be physically active due to health complaints or because they were wheelchair-bound. It would be good to inventory reasons why people do not perform a certain behaviour so that the advice can be made more personalized. 
Fifth, although almost $80 \%$ of our respondents who completed the process evaluation questionnaire reported they liked the use of traffic lights in the HRA, the use of traffic lights is questionable: the colour red (i.e. a warning colour) may discourage people. Other symbols could be used, for example smilies or hand signs.

Sixth, an environmental component could be added to our existing intervention. Our interventions were mainly based on individual characteristics. However, as shown in ecological approaches, the environment can also influence behaviour indirectly (Kremers, 2010). As presented by Van Stralen et al. (2011) and Peels et al. (2013), their environmentally computer-tailored print intervention was effective in increasing days and/or weekly minutes of physical activity. Environmental perceptions have been shown to be relevant as determinants of changing physical activity behaviour (Van Stralen et al., 2009b). Providing environmental information as an intervention strategy has also proven to be effective (Van Stralen et al., 2009b). Google maps could be used to give tailored information about, for example, sport facilities in the near neighbourhood (Prins et al., 2010).

Seventh, a multiple behaviour change intervention targeting lifestyle improvement should also aim at reducing sedentary behaviours (Helmink et al., 2013; Rutten et al., 2013). Maintaining healthy behaviour and relapse prevention are important, too. Interventions should focus more deeply on these aspects.

Finally, as a future direction for practice, third-generation computer-tailored interventions may be used (Norman et al., 2007). This means that a comparable programme could be developed and delivered via mobile phones (using tailored SMS) or smart phones and tablets (as applications). Tailored feedback messages could also be delivered via videos instead of or in addition to text-based messages (Stanczyk et al., 2011; Vandelanotte \& Mummery, 2011; Walthouwer et al., 2013). Results of a study by Stanczyk et al. (2014; submitted a; submitted b) indicated that the video condition of a web-based smoking cessation programme was more effective and more cost-effective after 12 months with regard to smoking cessation rates than the text condition and the control group.

\section{Implementing the interventions}

The multiple lifestyle intervention has already been adopted and implemented by the RHAs in Limburg, the Southern province of the Netherlands. The programme has the potential to be implemented nationally by all health authorities in the Netherlands (GGD Nederland), since the interface between the Adult Health Monitor and the web-based computer-tailored intervention has shown positive results regarding reach and (cost-)effectiveness. However, it is uncertain whether implementation via the RHAs is the most effective method in terms of reaching people. Further attention should be given to investigating how this type of intervention could be disseminated most optimally. Dissemination via GPs, for example, may be an alternative, since GPs' advice is well accepted by patients (Kreuter et al., 2000a). An earlier study by De Cocker et al. (2012) showed, for example, that three quarters of participants believed that it is useful for GPs to stimulate their patients in finding ways to be more physically active. However, recruitment via GPs was poor in their study 
(6.2\%), as well as in a study by Dickinson et al. (2013) (2.2\%). Thus, on the other hand, GPs' time and effort seem to be limited (Parekh et al., 2012). A mass media approach could also be applied. An earlier study has shown that a larger sample of smokers could be recruited via a mass media approach when compared to recruitment via general practices, against much lower costs (Smit et al., 2012b). Our lifestyle intervention could also be integrated in other national institutions, such as the Dutch Cancer Society, the Lung Foundation Netherlands or the Diabetes Federation, since these organizations also have the task of public health education. When offering our lifestyle intervention via the RHAs, completion of the online version of the Adult Health Monitor should be stimulated. In 2009, the written version of the Monitor was included in the envelope; this made it easy not to complete the online version which was linked to our web-based intervention.

The intervention can also be used as a stand-alone programme. An adapted version of our alcohol module is used by Mondriaan Zorggroep. This module aims at reaching problem drinkers and making them aware of their excessive alcohol use and the necessity to change their drinking behaviour. This website is open to the public. In 2013, 637 unique visits were registered. However, use of the tailored module was extremely low (in the year 2013: $n=5$ ). An explanation might be that registration is necessary before being allowed to complete the module.

The programme can also be used as a first-step intervention in a stepped-care approach (i.e. increasing type and intensity of interventions when people fail to change their behaviour), as also suggested by Riper et al. (2008). In this way, computer-tailored programmes can serve as a starting point in order to increase awareness of the problem (Oenema \& Brug, 2003). Computer-tailored programmes can also be used in combination with other interventions, such as counselling, and work as a supporting tool (e.g. Smit et al., 2010).

\section{GENERAL CONCLUSIONS}

This dissertation shows the promising effect of implementing a web-based computer-tailored lifestyle intervention on a large scale. Our intervention showed positive results regarding its reach, appreciation, effectiveness and cost-effectiveness. In a multiple behaviour change intervention, physical activity may serve as a gateway behaviour, since our physical activity module was preferred within the sequential condition. Both the sequential and the simultaneous tailoring strategy have advantages and disadvantages. A combination of both may be most suitable for multiple behaviour change. Within the single behaviour change intervention, the comparison between an alternating and a summative approach showed no indication that one of the tailoring strategies was more effective in reducing alcohol intake. However, the attrition rates during the first visit were lower in the alternating condition.

In general, the interventions suffered from high dropout rates. Therefore, the results have to be interpreted cautiously due to the high number of missing values. Moreover, selective dropout occurred. Much work remains to be done to understand the low levels of engagement in web-based 
interventions. Statistical methods for dealing with missing values need further attention. There is room for improvement of web-based computer-tailored interventions aimed at multiple behaviours. Combining and optimizing different tailoring strategies may help to increase their use and effectiveness. 


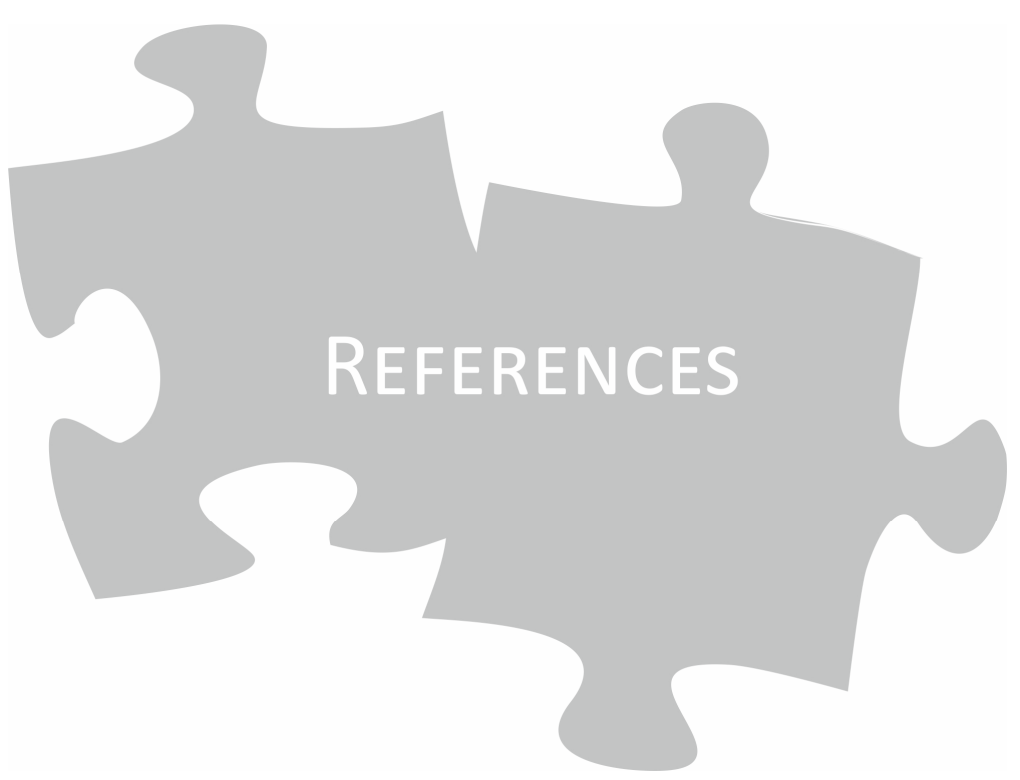





\section{A}

ABRAMS DB, ORLEANS CT, NIAURA RS, GOLDSTEIN MG, PROCHASKA JO, VELICER W. Integrating individual and public health perspectives for treatment of tobacco dependence under managed health care: a combined stepped-care and matching model. Ann Behav Med 1996; 18:290-304.

ADAMS J, WHITE M. Why don't stage-based activity promotion interventions work? Health Educ Res 2005; 20:237-243

AINSWORTH BE, HASKELL WL, WHITT MC, IRWIN ML, SWARTZ AM, STRATH SJ, O'BRIEN WL, BASSETT DR JR, SCHMITZ KH, EMPLAINCOURT PO, JACOBS DR JR, LEON AS. Compendium of physical activities: an update of activity codes and MET intensities. Med Sci Sports Exerc 2000; 32:498-504.

AJZEN I. The theory of planned behavior. Organ Behav Hum Decis Process 1991; 50:179-211.

ALAYLI-GOEBBELS A. Bringing two worlds closer together. Addressing methodological challenges in the economic evaluation of public health programs. Dissertation. Maastricht University, Maastricht; 2013.

ALLISON P. Statistical Horizons. Why you probably need more imputations than you think. 2012. URL: http://www.statisticalhorizons.com/more-imputations

ANDRESEN EM, MALMGREN JA, CARTER WB, PATRICK DL. Screening for depression in well older adults: evaluation of a short form of the CES-D (Center for Epidemiologic Studies Depression Scale). Am J Prev Med 1994; 10:77-84.

ANDREWS G, SLADE T. Interpreting scores on the Kessler psychological distress scale (K10). Aust N Z J Public Health 2001; 25:494-497.

ARAÚJO-SOARES V, MCINTYRE T, SNIEHOTTA FF. Predicting changes in physical activity among adolescents: the role of self-efficacy, intention, action planning and coping planning. Health Educ Res 2009; 24:128-139.

ATKINSON NL, SAPERSTEIN SL, PLEIS J. Using the internet for health-related activities: findings from a national probability sample. J Med Internet Res 2009; 11:e4.

AUSTIN JT, VANCOUVER JB. Goal constructs in psychology: Structure, process, and content. Psychol Bull 1996; 120:338-375.

AUSTRALIAN INSTITUTE OF HEALTH AND WELFARE (AIHW) 2002. Chronic Diseases and Associated Risk Factors in Australia, 2001. Canberra: AlHW.

B

BAKER AH, WARDLE J. Increasing fruit and vegetable intake among adults attending colorectal cancer screening: the efficacy of a brief tailored intervention. Cancer Epidemiol Biomarkers Prev 2002; 11:203-206.

BANDURA A. Social Foundations of Thought and Action: A Social Cognitive Theory. Englewood Cliffs, NJ: PrenticeHall; 1986.

BAUMEISTER RF. Ego depletion and self-regulation failure: a resource model of self-control. Alcohol Clin Res 2003; 27:281-284.

BAUMEISTER RF, BRATSLAVSKY E, MURAVEN M, TICE DM. Ego depletion: is the active self a limited resource? J Pers Soc Psychol 1998; 74:1252-1265. 
BENDTSEN P, MCCAMBRIDGE J, BENDTSEN M, KARLSSON N, NILSEN P. Effectiveness of a proactive mail-based alcohol Internet intervention for university students: dismantling the assessment and feedback components in a randomized controlled trial. J Med Internet Res 2012; 14:e142.

BENNETT GG, GLASGOW RE. The delivery of public health interventions via the Internet: actualizing their potential. Annu Rev Public Health 2009; 30:273-292.

BERGSTRÖM G, BJÖRKLUND C, FRIED I, LISSPERS J, NATHELL L, HERMANSSON U, HELANDER A, BODIN L, JENSEN IB. A comprehensive workplace intervention and its outcome with regard to lifestyle, health and sick leave: the AHA study. Work 2008; 31:167-180.

BERNDT NC. Smoking cessation in patients with coronary heart disease. Determinants of quitting and comparison of the (cost)effectiveness and feasibility of a telephone and face-to-face counseling intervention. Dissertation. Open University, Heerlen; 2013

BERRIGAN D, DODD K, TROIANO RP, KREBS-SMITH SM, BARBASH RB. Patterns of health behavior in U.S. adults. Prev Med 2003; 36:615-623.

BEWICK BM, TRUSLER K, BARKHAM M, HILL AJ, CAHILL J, MULHERN B. The effectiveness of web-based interventions designed to decrease alcohol consumption--a systematic review. Prev Med 2008; 47:17-26.

BEWICK BM, WEST R, GILL J, O'MAY F, MULHERN B, BARKHAM M, HILL AJ. Providing web-based feedback and social norms information to reduce student alcohol intake: a multisite investigation. J Med Internet Res 2010; 12:e59.

BLANKERS M, KOETER MW, SCHIPPERS GM. Missing data approaches in eHealth research: simulation study and a tutorial for nonmathematically inclined researchers. J Med Internet Res 2010; 12:e54.

BODNER TE. What Improves with Increased Missing Data Imputations? Structural Equation Modeling: A Multidisciplinary Journal 2008; 15:651-675.

BOGERS R, VAN ASSEMA P, KESTER A, WESTERTERP K, DAGNELIE P. Reproducibility, validity, and responsiveness to change of a sort questionnaire for measuring fruit and vegetable intake. Am J Epidemiol 2004; 159:900909.

BONNEAUX L, BIRNIE E. The discount rate in the economic evaluation of prevention: a thought experiment. J Epidemiol Community Health 2001; 55:123-125.

BOON B, RISSELADA A, HUIBERTS A, RIPER H, SMIT F. Curbing alcohol use in male adults through computer generated personalized advice: randomized controlled trial. J Med Internet Res 2011; 13:e43.

BOOTH AO, NOWSON CA, MATTERS H. Evaluation of an interactive, Internet-based weight loss program: a pilot study. Health Educ Res 2008; 23:371-381.

BORLAND R, BALMFORD J, HUNT D. The effectiveness of personally tailored computer-generated advice letters for smoking cessation. Addiction 2004; 99:369-377.

BROUWER W. Improving Exposure to Internet-Delivered Health Behavior Change Interventions. An Exploration of Determinants and Dissemination Strategies. Dissertation. Erasmus Universiteit Rotterdam, Rotterdam; 2011.

BROUWER W, KROEZE W, CRUTZEN R, DE NOOIJER J, DE VRIES NK, BRUG J, OENEMA A. Which intervention characteristics are related to more exposure to internet-delivered healthy lifestyle promotion interventions? A systematic review. J Med Internet Res 2011; 13:e2. 
BROUWER WB, NIESSEN LW, POSTMA MJ, RUTTEN FF. Need for differential discounting of costs and health effects in cost effectiveness analyses. BMJ 2005; 331:446-448.

BROUWER W, OENEMA A, CRUTZEN R, DE NOOIJER J, DE VRIES NK, BRUG J. An exploration of factors related to dissemination of and exposure to internet-delivered behavior change interventions aimed at adults: a Delphi study approach. J Med Internet Res 2008; 10:e10.

BROUWER W, OENEMA A, CRUTZEN R, DE NOOIJER J, DE VRIES N, BRUG J. What makes people decide to visit and use an internet-delivered behavior-change intervention?: A qualitative study among adults. Health Educ 2009; 109:460-473

BROUWER W, OENEMA A, RAAT H, CRUTZEN R, DE NOOIJER J, DE VRIES NK, BRUG J. Characteristics of visitors and revisitors to an Internet-delivered computer-tailored lifestyle intervention implemented for use by the general public. Health Educ Res 2010; 25:585-595.

BROUWER WBF, RUTTEN FFH. De hoogte van de disconteervoeten in economische evaluaties: Is de tijd rijp voor een nieuwe richtlijn? [The amount of the discount rate in economic evaluations: Has the time come for a new guideline?]. Rapportnummer 05.73. Erasmus MC, Institute for Medical Technology Assessment: Rotterdam; 2005.

BRUG J, CAMPBELL M, VAN ASSEMA P. The application and impact of computer-generated personalized nutrition education: a review of the literature. Pat Educ Couns 1999a; 36:145-156.

BRUG J, GLANZ K, VAN ASSEMA P, KOK G, VAN BREUKELEN GJ. The impact of computer-tailored feedback and iterative feedback on fat, fruit, and vegetable intake. Health Educ Behav 1998; 25:517-531.

BRUG J, OENEMA A, CAMPBELL M. Past, present, and future of computer-tailored nutrition education. Am J Clin Nutr 2003; 77:1028-1034.

BRUG J, OENEMA A, KROEZE W, RAAT H. The internet and nutrition education: challenges and opportunities. Eur J Clin Nutr 2005; 59:130-139.

BRUG J, STEENHUIS I, VAN ASSEMA P, GLANZ K, DE VRIES H. Computer-tailored nutrition education: differences between two interventions. Health Educ Res 1999b; 14:249-256.

BUDD RJ, ROLLNICK S. The structure of the Readiness to Change Questionnaire: A test of Prochaska and DiClemente's transtheoretical model. Br J Health Psychol 1997; 2:365-376.

BUNDORF MK, WAGNER TH, SINGER SJ, BAKER LC. Who searches the internet for health information? Health Serv Res 2006; 41:819-836.

BURTON TL, WILLIAMSON DL. Harmful effects of drinking and the use and perceived effectiveness of treatment. J Stud Alcohol 1995; 56:611-615.

C

CAMPBELL MK, DEVELLIS BM, STRECHER VJ, AMMERMAN AS, DEVELLIS RF, SANDLER RS. Improving dietary behaviors: the effectiveness of tailored messages in primary care settings. Am J Public Health 1994; 84:783787.

CAMPBELL MK, JAMES A, HUDSON MA, CARR C, JACKSON E, OAKES V, DEMISSIE S, FARRELL D, TESSARO I. Improving multiple behaviors for colorectal cancer prevention among African American church members. Health Psychol 2004; 23:492-502. 
CAMPBELL MK, TESSARO I, DEVELLIS B, BENEDICT S, KELSEY K, BELTON L, HENRIQUEZ-ROLDAN C. Tailoring and targeting a worksite health promotion program to address multiple health behaviors among blue-collar women. Am J Health Promot 2000; 14:306-313.

CARLSON JA, SALLIS JF, RAMIREZ ER, PATRICK K, NORMAN GJ. Physical activity and dietary behavior change in Internet-based weight loss interventions: comparing two multiple-behavior change indices. Prev Med 2012; 54:50-54.

CBO DUTCH INSTITUTE FOR HEALTHCARE IMPROVEMENT. Richtlijn behandeling van tabaksverslaving [Guidelines for treating tobacco addiction]. Utrecht/Den Haag: BVO/Partnership Stop met Roken; 2004.

CENTER FOR SUBSTANCE ABUSE TREATMENT. Enhancing Motivation for Change in Substance Abuse Treatment. Rockville (MD): Substance Abuse and Mental Health Services Administration (US); 1999. (Treatment Improvement Protocol (TIP) Series, No. 35.) URL: http://www.ncbi.nlm.nih.gov/books/NBK64967/

CHERPITEL CJ, BOND J, YE Y. Alcohol and injury: a risk function analysis from the Emergency Room Collaborative Alcohol Analysis Project (ERCAAP). Eur Addict Res 2006; 12:42-52.

CIVLJAK M, SHEIKH A, STEAD LF, CAR J. Internet-based interventions for smoking cessation. Cochrane Database Syst Rev 2010; 9:CD007078.

CLAXTON K, PAULDEN M, GRAVELLE H, BROUWER W, CULYER AJ. Discounting and decision making in the economic evaluation of health-care technologies. Health Econ 2011; 20:2-15.

COBIAC LJ, VOS T, BARENDREGT JJ. Cost-effectiveness of interventions to promote physical actvity: a modelling study. PloS Medicine 2009; 6:e1000111.

COHEN J. Statistical Power Analysis for the Behavioral Sciences (second ed.). Hillsdale, N.J.: L. Erlbaum Associates; 1988.

COHEN J. The earth is round $(p<.05)$. Am Psychol 1994; 49:997-1003.

COUNCIL FOR PUBLIC HEALTH AND CARE (RAAD VOOR DE VOLKSGEZONDHEID EN ZORG, RVZ). Zinnige en duurzame zorg. 2006. URL: http://www.rvz.net/uploads/docs/Advies_-_Zinnige_en_duurzame_zorg.pdf

CRUTZEN R. Hard to Get, Hard to Keep. Dissemination of and Exposure to Internet-Delivered Health Behavior Change Interventions Aimed at Adolescents. Dissertation. Maastricht University, Maastricht; 2009.

CRUTZEN R, CYR D, DE VRIES NK. Bringing loyalty to e-Health: theory validation using three internet-delivered interventions. J Med Internet Res 2011; 13:e73.

CRUTZEN R, CYR D, DE VRIES NK. The role of user control in adherence to and knowledge gained from a website: randomized comparison between a tunneled version and a freedom-of-choice version. J Med Internet Res 2012; 14:e45.

CUNNINGHAM JA. Comparison of two internet-based interventions for problem drinkers: randomized controlled trial. J Med Internet Res 2012; 14:e107.

CUNNINGHAM JA, BRESLIN FC. Only one in three people with alcohol abuse or dependence ever seek treatment. Addict Behav 2004; 29:221-223.

CUNNINGHAM JA, WILD TC, CORDINGLEY J, VAN MIERLO T, HUMPHREYS K. A randomized controlled trial of an internet-based intervention for alcohol abusers. Addiction 2009; 104:2023-2032. 
CUNNINGHAM JA, WILD TC, CORDINGLEY J, VAN MIERLO T, HUMPHREYS K. Twelve-month follow-up results from a randomized controlled trial of a brief personalized feedback intervention for problem drinkers. Alcohol Alcohol 2010; 45:258-262.

D

DANAHER BG, SMOLKOWSKI K, SEELEY JR, SEVERSON HH. Mediators of a successful web-based smokeless tobacco cessation program. Addiction 2008; 103:1706-1712.

DAVIES C, SPENCE J, VANDELANOTTE C, CAPERCHIONE C, MUMMERY K. Meta-analysis of Internet-delivered interventions to increase physical activity. Int J Behav Nutr Phys Act 2012a; 9:52.

DAVIES CA, VANDELANOTTE C, DUNCAN MJ, VAN UFFELEN JG. Associations of physical activity and screen-time on health related quality of life in adults. Prev Med 2012b; 55:46-49.

DAVIS JC, BRYAN S, MARRA CA, SHARMA D, CHAN A, BEATTIE BL, GRAF P, LIU-AMBROSE T. An economic evaluation of resistance training and aerobic training versus balance and toning exercises in older adults with mild cognitive impairment. PLoS One 2013; 8:e63031.

DECI EL, RYAN RM. Intrinsic motivation and self-determination in human behavior. New York: Plenum; 1985.

DECI EL, RYAN RM. The "what" and "why" of goal pursuits: Human needs and the self-determination of behavior. Psychological Inquiry 2000; 11:227-268.

DE COCKER K, SPITTAELS H, CARDON G, DE BOURDEAUDHUIJ I, VANDELANOTTE C. Web-based, computertailored, pedometer-based physical activity advice: development, dissemination through general practice, acceptability, and preliminary efficacy in a randomized controlled trial. J Med Internet Res 2012; 14:e53.

DE NOOIJER J, OENEMA A, KLOEK G, BRUG H, DE VRIES H. Bevordering van gezond gedrag via het internet: nu en in de toekomst [Promotion of healthy behavior through the Internet: Now and in the future]. Rotterdam: Optima Grafische Communicatie; 2005.

DE VET E, BRUG J, DE NOOIJER J, DIJKSTRA A, DE VRIES NK. Determinants of forward stage transitions: a Delphi study. Health Educ Res 2005; 20:195-205.

DE VET E, DE NOOIJER J, DE VRIES NK, BRUG J. The Transtheoretical model for fruit, vegetable and fish consumption: associations between intakes, stages of change and stage transition determinants. Int J Behav Nutr Phys Act 2006; 3:13.

DE VET E, GEBHARDT WA, SINNIGE J, VAN PUFFELEN A, VAN LETTOW B, DE WIT JB. Implementation intentions for buying, carrying, discussing and using condoms: the role of the quality of plans. Health Educ Res 2011; 26:443-455.

DE VRIES H, BACKBIER E. Self-efficacy as an important determinant of quitting among pregnant women who smoke: the $\varnothing$ - pattern. Prev Med 1994; 23:167-174.

DE VRIES H, BRUG J. Computer-tailored interventions motivating people to adopt health promoting behaviours: introduction to a new approach. Patient Educ Couns 1999; 36:99-105.

DE VRIES H, DIJKSTRA M, KUHLMAN P. Self-efficacy: the third factor besides attitude and subjective norm as a predictor of behavioral intentions. Health Educ Res 1988; 3:273-282.

DE VRIES H, EGGERS SM, BOLMAN C. The role of action planning and plan enactment for smoking cessation. BMC Public Health 2013; 13:393. 
DE VRIES H, KREMERS S, SMEETS T, BRUG J, EIJMAEL K. The effectiveness of tailored feedback and action plans in an intervention addressing multiple health behaviors. Am J Health Promot 2008a; 22:417-425.

DE VRIES H, KREMERS S, SMEETS T, REUBSEAT A. Clustering of diet, physical activity and smoking and a general willingness to change. Psychol Health 2008b; 23:18-24.

DE VRIES H, LEZWIJN J, HOL M, HONING C. Skin cancer prevention: behaviour and motives of Dutch adolescents. Eur J Cancer Prev 2005; 14:39-50.

DE VRIES H, MUDDE AN, LEIJS I, CHARLTON A, VARIAINEN E, BUIJS G, PAIS CLEMENTE M, STORM H, GONZÁLEZ NAVARRO A, NEBOT M, PRINS T, KREMERS S. The European Smoking Prevention Framework Approach (EFSA): an example of integral prevention. Health Educ Res 2003; 18:611-626.

DE VRIES H, VAN ‘ T RIET J, SPIGT M, METSEMAKERS J, VAN DEN AKKER M, VERMUNT JK, KREMERS S. Clusters of lifestyle behaviors: results from the Dutch SMILE study. Prev Med 2008c; 46:203-208.

DICKINSON WP, GLASGOW RE, FISHER L, DICKINSON LM, CHRISTENSEN SM, ESTABROOKS PA, MILLER BF. Use of a website to accomplish health behavior change: if you build it, will they come? And will it work if they do? J Am Board Fam Med 2013; 26:168-176.

DIJKSTRA A. Working mechanisms of computer-tailored health education: evidence from smoking cessation. Health Educ Res 2005; 20:527-539.

DIJKSTRA A, DE VRIES H. The development of computer-generated tailored interventions. Pat Educ Couns 1999; 36:193-203.

DIJKSTRA A, DE VRIES H, ROIJACKERS J. Long-term effectiveness of computer-generated tailored feedback in smoking cessation. Health Educ Res 1998a; 13:207-214.

DIJKSTRA A, DE VRIES H, ROIJACKERS J, VAN BREUKELEN G. Tailored interventions to communicate stagematched information to smokers in different motivational stages. J Consult Clin Psychol 1998b; 3:549-557.

DIJKSTRA A, DE VRIES H, ROIJACKERS J, VAN BREUKELEN G. Tailoring information to enhance quitting in smokers with low motivation to quit: three basic efficacy questions. Health Psychol 1998c; 17:513-519.

DOLL R, PETO R. The causes of cancer: quantitative estimates of avoidable risks of cancer in the United States today. J Natl Cancer Inst 1981; 66:1191-1308.

DRUMMOND, MF. Methods for the economic evaluation of health care programmes. Oxford: Oxford University Press; 1997.

DRUMMOND MF, SCULPHER MJ, TORRANCE GW, O'Brien BJ, Stoddart GL. Methods for the economic evaluation of health care programmes. Third edition. Oxford University Press: New York; 2005.

DUNN CL, HANNAN PJ, JEFFERY RW, SHERWOOD NE, PRONK NP, BOYLE R. The comparative and cumulative effects of a dietary restriction and exercise on weight loss. Int J Obes (Lond) 2006; 30:112-121.

DUTTA-BERGMAN MJ. Health attitudes, health cognitions, and health behaviors among Internet health information seekers: population-based survey. J Med Internet Res 2004; 6:e15.

DUTTON GR, NAPOLITANO MA, WHITELEY JA, MARCUS BH. Is physical activity a gateway behavior for diet? Findings from a physical activity trial. Prev Med 2008; 46:216-221. 


\section{$E$}

EARLEY PC, WOJNAROSKI P, PREST W. Task planning and energy expended: Exploration of how goals influence performance. J Appl Psychol 1987; 72:107-114.

EDINGTON DW. Emerging research: a view from one research center. Am J Health Promot 2001; 15:341-349.

ELFEDDALI I, BOLMAN C, CANDEL MJ, WIERS RW, DE VRIES H. Preventing smoking relapse via Web-based computer-tailored feedback: a randomized controlled trial. J Med Internet Res 2012a; 14:e109.

ELFEDDALI I, BOLMAN C, CANDEL MJ, WIERS RW, DE VRIES H. The role of self-efficacy, recovery self-efficacy, and preparatory planning in predicting short-term smoking relapse. Br J Health Psychol 2012b; 17:185-201.

ENDERS CK. Applied Missing Data Analysis. Methodology in the social sciences. New York: Guilford Press; 2010.

ETTER JF, SUTTON S. Assessing " stage of change " in current and former smokers. Addiction 2002; 97:11711182.

EUROQOL GROUP. EuroQol-- a new facility for the measurement of health-related quality of life. Health Policy 1990; 16:199-208.

EVERS KE, CUMMINS CO, PROCHASKA JO, PROCHASKA JM. Online health behavior and disease management programs: are we ready for them? Are they ready for us? J Med Internet Res 2005; 7:e27.

EVERS KE, PROCHASKA JM, PROCHASKA JO, DRISKELL MM, CUMMINS CO, VELICER WF. Strengths and weaknesses of health behavior change programs on the internet. J Health Psychol 2003; 8:63-70.

EYSENBACH G: The law of attrition. J Med Internet Res 2005; 7:e11.

EYSENBACH G, CONSORT-EHEALTH GROUP. CONSORT-EHEALTH. Improving and Standardizing Evaluation Reports of Web-based and Mobile Health Interventions. J Med Internet Res 2011; 13:e126.

EZZATI M, HOORN SV, RODGERS A, LOPEZ AD, MATHERS CD, MURRAY CJ, COMPARATIVE RISK ASSESSMENT COLLABORATING GROUP. Estimates of global and regional potential health gains from reducing multiple major risk factors. Lancet 2003; 362:271-280.

EZZATI M, LOPEZ AD, RODGERS A, VANDER HOORN S, MURRAY CJ, COMPARATIVE RISK ASSESSMENT COLLABORATING GROUP. Selected major risk factors and global and regional burden of disease. Lancet 2002; 360:1347-1360.

$\mathrm{F}$

FARKAS AJ, PIERCE JP, ZHU SH, ROSBROOK B, GILPIN EA, BERRY C, KAPLAN RM. Addiction versus stages of change models in predicting smoking cessation. Addiction 1996; 91:1271-1280.

FEENSTRA TL, HAMBERG-VAN REENEN HH, HOOGENVEEN RT, RUTTEN-VAN MÖLKEN MP. Cost-effectiveness of face-to-face smoking cessation interventions: a dynamic modeling study. Value Health 2005; 8:178-190.

FINE LJ, PHILOGENE GS, GRAMLING R, COUPS EJ, SINHA S. Prevalence of multiple chronic disease risk factors. 2001 National Health Interview Survey. Am J Prev Med 2004; 27:18-24.

FOX S. Health topics: $80 \%$ of Internet users look for health information online. Washington, DC: Pew Internet \& American Life Project; 2011. URL: http://www.webcitation.org/65hAc9yrr 
FRICK A, BÄCHTIGER M T, REIPS U-D. Financial incentives, personal information, and drop out in online studies. In: Reips U-D, Bosnjak M (editors). Dimensions of Internet science (pp. 209-219). Lengerich, Germany: Pabst Science; 2001.

FRIEDERICHS SA, OENEMA A, BOLMAN C, GUYAUX J, VAN KEULEN HM, LECHNER L. Motivational interviewing in a web-based physical activity intervention: questions and reflections. Health Promot Int 2013

\section{G}

GEELS LM, VINK JM, VAN BEEK JH, BARTELS M, WILLEMSEN G, BOOMSMA DI. Increases in alcohol consumption in women and elderly groups: evidence from an epidemiological study. BMC Public Health 2013; 13:207.

GERHARDS SA, DE GRAAF LE, JACOBS LE, SEVERENS JL, HUIBERS MJ, ARNTZ A, RIPER H, WIDDERSHOVEN G, METSEMAKERS JF, EVERS SM. Economic evaluation of online computerised cognitive-behavioural therapy without support for depression in primary care: randomised trial. Br J Psychiatry 2010; 196:310-318.

GEURTS M, VAN ROSSUM CTM. Voeding: Zijn er verschillen naar sociaaleconomische status? [Nutrition: Are there differences in socioeconomic status?] In: Volksgezondheid Toekomst Verkenning, Nationaal Kompas $\begin{array}{llll}\text { Volksgezondheid. } \quad \text { Bilthoven: } & 2013 .\end{array}$ http://www.nationaalkompas.nl/gezondheidsdeterminanten/leefstijl/voeding/

GLASGOW RE. eHealth evaluation and dissemination research. Am J Prev Med 2007; 32:119-126.

GLASGOW RE, GOLDSTEIN MG, OCKENE JK, PRONK NP. Translating what we have learned into practice. Principles and hypotheses for interventions addressing multiple behaviors in primary care. Am J Prev Med 2004; 27:88-101.

GLASGOW RE, NELSON CC, KEARNEY KA, REID R, RITZWOLLER DP, STRECHER VJ, COUPER MP, GREEN B, WILDENHAUS K. Reach, engagement, and retention in an Internet-based weight loss program in a multi-site randomized controlled trial. J Med Internet Res 2007; 9:e11.

GLASGOW RE, VOGT TM, BOLES SM. Evaluating the public health impact of health promotion interventions: the RE-AIM framework. Am J Public Health 1999; 89:1322-1327.

GODIN G, BÉLANGER-GRAVEL A, AMIREAULT S, VOHL MC, PÉRUSSE L. The effect of mere-measurement of cognitions on physical activity behavior: a randomized controlled trial among overweight and obese individuals. Int J Behav Nutr Phys Act 2011; 8:2.

GOMMER AM, POOS MJJC. Welke ziekten hebben de hoogste prevalentie? [Which disesases have the highest prevalance?] In: Volksgezondheid Toekomst Verkenning, Nationaal Kompas Volksgezondheid. Bilthoven: RIVM; 2010. URL: http://www.nationaalkompas.nl/gezondheid-en-ziekte/ziekten-en-aandoeningen/

GOOSSENS ME, RUTTEN-VAN MÖLKEN MP, VLAEYEN JW, VAN DER LINDEN SM. The cost diary: a method to measure direct and indirect costs in cost-effectiveness research. J Clin Epidemiol 2000; 53:688-695.

GRAHAM JW, OLCHOWSKI AE, GILREATH TD. How many imputations are really needed? Some practical clarifications of multiple imputation theory. Prev Sci 2007; 8:206-213.

GRAVELLE H, BROUWER W, NIESSEN L, POSTMA M, RUTTEN F. Discounting in economic evaluations: stepping forward towards optimal decision rules. Health Econ 2007; 16:307-317.

GRAVELLE H, SMITH D. Discounting for health effects in cost-benefit and cost-effectiveness analysis. Health Econ $2001 ; 10: 587-599$. 
GRIFFITHS F, LINDENMEYER A, POWELL J, LOWE P, THOROGOOD M. Why are health care interventions delivered over the internet? A systematic review of the published literature. J Med Internet Res 2006; 8:e10.

GUILLAUMIE L, GODIN G, MANDERSCHEID JC, SPITZ E, MULLER L. The impact of self-efficacy and implementation intentions-based interventions on fruit and vegetable intake among adults. Psychol Health 2012; 27:30-50.

$\mathrm{H}$

HACKSHAW A, KIRKWOOD A. Interpreting and reporting clinical trials with results of borderline significance. BMJ 2011; 343:d3340.

HAGGER MS, WOOD C, STIFF C, CHATZISARANTIS NL. Ego depletion and the strength model of self-control: a meta-analysis. Psychol Bull 2010; 136:495-525.

HAKKAART-VAN ROIJEN L, TAN SS, BOUWMANS C. Handleiding voor kostenonderzoek: Methoden en standaard kostprijzen voor economische evaluaties in de gezondheidszorg [Manual for cost research: Methods and standard costprizes for economic evaluation in the health care]. 2010. URL: http://www.cvz.nl/binaries/content/documents/zinl-www/documenten/publicaties/overigepublicaties/1007-handleiding-voor-kostenonderzoek/Handleiding+voor+kostenonderzoek.pdf.

HANSEN AB, BECKER U, NIELSEN AS, GRØNBAEK M, TOLSTRUP JS, THYGESEN LC. Internet-based brief personalized feedback intervention in a non-treatment-seeking population of adult heavy drinkers: a randomized controlled trial. J Med Internet Res 2012; 14:e98.

HANSEN AW, GRØNBÆEK M, HELGE JW, SEVERIN M, CURTIS T, TOLSTRUP JS. Effect of a Web-Based Intervention to Promote Physical Activity and Improve Health Among Physically Inactive Adults: A Population-Based Randomized Controlled Trial. J Med Internet Res 2012; 14:e145.

HARRIS JR, HUANG Y, HANNON PA, WILLIAMS B. Low-socioeconomic status workers: their health risks and how to reach them. J Occup Environ Med 2011; 53:132-138.

HAUG S, SANNEMANN J, MEYER C, JOHN U. Reduktion des Alkoholkonsums und Förderung der Rauchabstinenz bei Jugendlichen und jungen Erwachsenen mittels Internet und Mobiltelefon: ein Literaturüberblick [Internet and mobile phone interventions to decrease alcohol consumption and to support smoking cessation in adolescents: a review]. Gesundheitswesen 2012; 74:160-177.

HAWKES AL, CHAMBERS SK, PAKENHAM KI, PATRAO TA, BAADE PD, LYNCH BM, AITKEN JF, MENG X, COURNEYA KS. Effects of a Telephone-Delivered Multiple Health Behavior Change Intervention (CanChange) on Health and Behavioral Outcomes in Survivors of Colorectal Cancer: A Randomized Controlled Trial. J Clin Oncol 2013; $31: 2313-2321$.

HEALTH CARE INSURANCE BOARD (College voor Zorgverzekeringen, CVZ). Richtlijnen voor farmaco-economisch onderzoek; geactualiseerde versie [Guidelines for pharmacoeconomic research, actualized version]. Diemen; 2006. URL: http://www.webcitation.org/6LXwXi72v

HEALTH CARE INSURANCE BOARD (College van Zorgverzekeringen, CVZ). Medicijnkosten [Medicine costs]. 2013. URL: www.medicijnkosten.nl

HEATHERTON TF, KOZLOWSKI LT, FRECKER RC, FAGERSTRÖM KO. The Fagerström Test for Nicotine Dependence: a revision of the Fagerström Tolerance Questionnaire. Br J Addict 1991; 86:1119-1127. 
HELMINK JH, GUBBELS JS, VAN BRUSSEL-VISSER FN, DE VRIES NK, KREMERS SP. Baseline predictors of maintenance of intervention-induced changes in physical activity and sitting time among diabetic and prediabetic patients: a descriptive case series. BMC Res Notes 2013; 6:190.

HEMP P. Presenteeism: at work--but out of it. Harv Bus Rev 2004; 82:49-58, 155.

HENDRIKS MR, AL MJ, BLEIJLEVENS MH, VAN HAASTREGT JC, CREBOLDER HF, VAN EIJK JT, EVERS SM. Continuous versus Intermittent Data Collection of Health Care Utilization. Med Decis Making 2013; 33:998-1008.

HERZOG TA, ABRAMS DB, EMMONS KM, LINNAN L, SHADEL WG. Do processes of change predict smoking stage movements? A prospective analysis of the Transtheoretical Model. Health Psychol 1999; 4:369-375.

HESTER RK, DELANEY HD, CAMPBELL W, HANDMAKER N. A web application for moderation training: initial results of a randomized clinical trial. J Subst Abuse Treat 2009; 37:266-276.

HILDEBRANDT V, BERNAARDS C, CHORUS A, HOFSTETTER H. Bewegen in Nederland 2000-2011 [Exercising in the Netherlands 2000-2011]. In: Trendrapport Bewegen en Gezondheid 2010/2011. Leiden: TNO; 2013.

HILDEBRANDT VH, CHORUS AMJ, STUBBE JH. Trendrapport Bewegen en Gezondheid 2008-2009 [Trend Report on Physical Activity and Health 2008-2009]. TNO Kwaliteit van Leven. Leiden; 2010.

HOEYMANS N, SCHELLEVIS FC, WOLTERS I. Hoeveel mensen hebben één of meer chronische ziekten? [How many people have one or more chronic diseases?] In: Volksgezondheid Toekomst Verkenning, Nationaal Kompas Volksgezondheid. Bilthoven: RIVM; 2008. URL: http://www.nationaalkompas.nl/gezondheid-enziekte/ziekten-en-aandoeningen/chronische-ziekten-en-multimorbiditeit/

HYMAN DJ, PAVLIK VN, TAYLOR WC, GOODRICK GK, MOYE L. Simultaneous vs sequential counseling for multiple behaviour change. Arch Intern Med 2007; 167:1152-1158.

|

INTERNET WORLD STATS. Internet usage statistics: The internet big picture. Usage and population statistics. Internet usage in Europe. Internet user statistics and population for 53 European countries and regions. 2012. URL: http://www.internetworldstats.com/stats4.htm

IRVINE AB, ARY DV, GROVE DA, GILFILLAN-MORTON L. The effectiveness of an interactive multimedia programme to influence eating habits. Health Educ Res 2004; 19:290-305.

J

JACOBS AD, AMMERMAN AS, ENNETT ST, CAMPBELL MK, TAWNEY KW, AYTUR SA, MARSHALL SW, WILL JC, ROSAMOND WD. Effects of a tailored follow-up intervention on health behaviors, beliefs and attitudes. J Womens Health (Larchmt) 2004; 13:557-568.

JAKICIC JM, WING RR, WINTERS-HART C. Relationship of physical activity to eating behaviors and weight loss in women. Med Sci Sports Exerc 2002; 34:1653-1659.

JANZ NK, BECKER MH. The Health Belief Model: a decade later. Health Educ Q 1984; 11:1-47.

JENKINSON C, LAYTE R, JENKINSON D, LAWRENCE K, PETERSEN S, PAICE C, STRADLING J. A shorter form health survey: can the SF-12 replicate results from the SF-36 in longitudinal studies? J Public Health Med 1997; 19:179-186. 
JENSEN MK, S $\varnothing$ RENSEN TI, ANDERSEN AT, THORSEN T, TOLSTRUP JS, GODTFREDSEN NS, GR ØNBAEK M. A prospective study of the association between smoking and later alcohol drinking in the general population. Addiction 2003; 98:355-363.

JIAO L, MITROU PN, REEDY J, GRAUBARD BI, HOLLENBECK AR, SCHATZKIN A, STOLZENBERG-SOLOMON R. A combined healthy lifestyle score and risk of pancreatic cancer in a large cohort study. Arch Intern Med 2009; 169:764-770

JOHNSON MF, NICHOLS JF, SALLIS JF, CALFAS KJ, HOVELL MF. Interrelationships between physical activity and other health behaviors among university women and men. Prev Med 1998; 27:536-544.

JÖNSSEN B. Ten arguments for a societal perspective in the economic evaluation of medical innovations. Eur J Health Econ 2009; 10:357-359.

K

KARANIKOLOS M, MLADOVSKY P, CYLUS J, THOMSON S, BASU S, STUCKLER D, MACKENBACH JP, MCKEE M. Financial crisis, austerity, and health in Europe. Lancet 2013; 381:1323-1331.

KEMPER HGC, OOIJENDIJK WTM, STIGGELBOUT M. Consensus over de Nederlandse Norm voor Gezond Bewegen [Consensus on the Dutch standard for healthy exercise]. TSG 2000; 78:180-183.

KESSLER RC, ANDREWS G, COLPE LJ, HIRIPI E, MROCZEK DK, NORMAND SL, WALTERS EE, ZASLAVSKY AM. Short screening scales to monitor population prevalences and trends in non-specific psychological distress. Psychol Med 2002; 32:959-976.

KESSLER R, MROCZEK D. Final Versions of our Non-Specific Psychological Distress Scale [Written communication memo dated 10/3/94] Ann Arbor (MI), Survey Research Center of the Institute for Social Research, University of Michigan; 1994.

KING AC, CASTRO CM, BUMAN MP, HEKLER EB, URIZAR GG JR, AHN DK. Behavioral impacts of sequentially versus simultaneously delivered dietary plus physical activity interventions: the CALM Trial. Ann Behav Med 2013; 46:157-168

KING TK, MARCUS BH, PINTO BM, EMMONS KM, ABRAMS DB. Cognitive-behavioral mediators of changing multiple behaviors: smoking and a sedentary lifestyle. Prev Med 1996; 25:684-691.

KOHL LF, CRUTZEN R, DE VRIES NK. Online prevention aimed at lifestyle behaviors: a systematic review of reviews. J Med Internet Res 2013; 15:e146.

KOPEC JA, WILLISON KD. A comparative review of four preference-weighted measures of health-related quality of life. J Clin Epidemiol 2003; 56:317-325.

KOSHY P, MACKENZIE M, LESLIE W, LEAN M, HANKEY C. Eating the elephant whole or in slices: views of participants in a smoking cessation intervention trial on multiple behaviour changes as sequential or concurrent tasks. BMC Public Health 2012; 12:500.

KOSMA M, CARDINAL BJ, MCCUBBIN JA. A pilot study of a web-based physical activity motivational program for adults with physical disabilities. Disabil Rehabil 2005; 27:1435-1442.

KREBS P, PROCHASKA JO, ROSSI JS. A meta-analysis of computer-tailored interventions for health behavior change. Prev Med 2010; 51:214-221. 
KREMERS SP. Theory and practice in the study of influences on energy balance-related behaviors. Patient Educ Couns 2010; 79:291-298.

KREMERS SPJ, DE BRUIJN GJ, SCHAALMA H, BRUG J. Clustering of energy balance-related behaviours and their intrapersonal determinants. Psychol Health 2004; 19:595-606.

KREMERS SPJ, VISSCHER TLS, BRUG J, CHIN A PAW MJ, SCHOUTEN EG, SCHUIT AJ, SEIDELL JC, VAN BAAK MA, VAN MECHELEN W, KEMPER HC, KOK FJ, SARIS WH, KROMHOUT D. Netherlands research programme weight gain prevention (NHF-NRG): rationale, objectives and strategies. Eur J Clin Nutr 2005; 59:498-507.

KREUTER MW, BULL FC, CLARK EM, OSWALD DL. Understanding how people process health information: a comparison of tailored and untailored weight loss materials. Health Psychol 1999a; 18:487-494.

KREUTER MW, CHHEDA SG, BULL FC. How does physician advice influence patient behavior? Evidence for a priming effect. Arch Fam Med 2000a; 9:426-433.

KREUTER MW, FARRELL D, OLEVITCH L, BRENNAN L. Tailored Health Messages. Customizing Communication with Computer Technology. Mahwah, New Jersey: Lawrence Erlbaum Associates; 1999b.

KREUTER MW, OSWALD DL, BULL FC, CLARK EM. Are tailored health education materials always more effective than non-tailored materials? Health Educ Res 2000b; 15:305-315.

KREUTER MW, SKINNER CS. Tailoring: what's in a name? Health Educ Res 2000; 15:1-4.

KREUTER MW, WRAY RJ. Tailored and targeted health communication: strategies for enhancing information relevance. Am J Health Behav 2003; 27:227-232.

KUNTSCHE E, REHM J, GMEL G. Characteristics of binge drinkers in Europe. Soc Sci Med 2004; 59:113-127.

KUSHNER RF, SORENSEN KW. Lifestyle medicine: the future of chronic disease management. Curr Opin Endocrinol Diabetes Obes 2013; 20:389-395

KWAK L, KREMERS SP, CANDEL MJ, VISSCHER TL, BRUG J, VAN BAAK MA. Changes in skinfold thickness and waist circumference after 12 and 24 months resulting from the NHF-NRG In Balance-project. Int J Behav Nutr Phys Act 2010; 7:26.

KWAK L, KREMERS SP, WERKMAN A, VISSCHER TL, VAN BAAK MA, BRUG J. The NHF-NRG In Balance-project: the application of Intervention Mapping in the development, implementation and evaluation of weight gain prevention at the worksite. Obes Rev 2007; 8:347-361.

KYPRI K, MCANALLY HM. Randomized controlled trial of a web-based primary care intervention for multiple health risk behaviors. Prev Med 2005; 41:761-766.

KYPRI K, SITHARTHAN T, CUNNINGHAM JA, KAVANAGH DJ, DEAN JI. Innovative approaches to intervention for problem drinking. Curr Opin Psychiatry 2005; 18:229-234.

LAMERS LM, STALMEIER PF, MCDONNELL J, KRABBE PF, VAN BUSSCHBACH JJ. Kwaliteit van leven meten in economische evaluaties: het Nederlands EQ-5D-tarief [Measuring the quality of life in economic evaluations: the Dutch EQ-5D tariff]. Ned Tijdschr Geneeskd 2005; 149:1574-1578.

LAMPERT T. Smoking, physical inactivity, and obesity: associations with social status. Dtsch Arztebl Int 2010; 107:1-7. 
LEFEBVRE RC, TADA Y, HILFIKER SW, BAUR C. The assessment of user engagement with eHealth content: the eHealth engagement scale. J Comput Mediat Commun 2010; 15:666-681.

LEMMENS P, TAN ES, KNIBBE RA. Measuring quantity and frequency of drinking in a general population survey: a comparison of five indices. J Stud Alcohol 1992; 53:476-486.

LESLIE E, MARSHALL AL, OWEN N, BAUMAN A. Engagement and retention of participants in a physical activity website. Prev Med 2005; 40:54-59.

LITTLE RJA, RUBIN DB. Statistical analysis with missing data. Second edition. Hoboken, NJ: Wiley; 2002.

LORENCE D, PARK H. Group disparities and health information: a study of online access for the underserved. Health Informatics J 2008; 14:29-38.

LORGELLY PK, LAWSON KD, FENWICK EA, BRIGGS AH. Outcome measurement in economic evaluations of public health interventions: a role for the capability approach? Int J Environ Res Public Health 2010; 7:2274-2289.

LUSTRIA ML, CORTESE J, NOAR SM, GLUECKAUF RL. Computer-tailored health interventions delivered over the web: review and analysis of key components. Patient Educ Couns 2009; 74:156 -173.

LUSTRIA ML, NOAR SM, CORTESE J, VAN STEE SK, GLUECKAUF RL, LEE J. A meta-analysis of web-delivered tailored health behavior change interventions. J Health Commun 2013; 18:1039-1069.

\section{$\mathrm{M}$}

MANCA A, HAWKINS N, SCULPHER MJ. Estimating mean QALYs in trial-based cost-effectiveness analysis: the importance of controlling for baseline utility. Health Econ 2005; 14:487-496.

MARCUS BH, BOCK BC, PINTO BM, FORSYTH LH, ROBERTS MB, TRAFICANTE RM. Efficacy of an individualized, motivationally-tailored physical activity intervention. Ann Behav Med 1998; 20:174-180.

MARKLAND D, VANSTEENKISTE M. Self determination theory and motivational interviewing in exercise. In: Hagger MS, Chatzisarantis N (editors). Intrinsic Motivation and Self-Determination in Exercise and Sport. Champaign, IL: Human Kinetics; 2007: 87-99.

MATA J, SILVA MN, VIEIRA PN, CARRACA EV, ANDRADE AM, COUTINHO SR, SARDINHA LB, TEIXEIRA PJ. Motivational "Spill-Over" during weight control: increased self-determination and exercise intrinsic motivation predict eating self-regulation. Health Psychol 2009; 28:709-716.

MATANO RA, KOOPMAN C, WANAT SF, WINZELBERG AJ, WHITSELL SD, WESTRUP D, FUTA K, CLAYTON JB, MUSSMAN L, TAYLOR CB. A pilot study of an interactive website in the workplace for reducing alcohol consumption. J Subst Abuse Treat 2007; 32:71-80.

MEICHENBAUM D, TURK DC. Facilitating treatment adherence: a practitioner's guidebook. New York: Plenum Press; 1987.

MENG L, MASKARINEC G, LEE J, KOLONEL LN. Lifestyle factors and chronic diseases: application of a composite risk index. Prev Med 1999; 29:296-304.

MILLER WR, ROLLNICK S. Motivational Interviewing: Preparing People to Change Addictive Behavior. New York: Guilford Press; 1995.

MILLER WR, ROLLNICK S. Ten things that motivational interviewing is not. Behav Cogn Psychother 2009; 37:129140. 
MONCUR RA, LARMER JC. Clinical applicability of intention-to-treat analyses. Evidence Based Med 2009; 6:39-41.

MORETTI FA, DE OLIVEIRA VE, KOGA DA SILVA EM. Access to health information on the internet: a public health issue? Rev Assoc Med Bras 2012; 58:650-658.

MUDDE AN, WILLEMSEN MC, KREMERS S, DE VRIES H. Meetinstrumenten voor onderzoek naar roken en stoppen met roken [Measuring instruments for research regarding smoking and smoking cessation]. The Hague, STIVORO; 2006.

MULLAN B, WONG C, ALLOM V, PACK SL. The role of executive function in bridging the intention-behaviour gap for binge-drinking in university students. Addict Behav 2011; 36:1023-1026.

MURAVEN M, SLESSAREVA E. Mechanisms of self-control failure: motivation and limited resources. Pers Soc Psychol Bull 2003; 29:894-906.

MURGRAFF V, PARROTT A, BENNETT P. Risky single-occasion drinking amongst young people--definition, correlates, policy, and intervention: a broad overview of research findings. Alcohol Alcohol 1999; 34:3-14.

\section{$\mathrm{N}$}

NATIONAL GOVERNMENT. Landelijke nota gezondheidsbeleid. Gezondheid dichtbij [National note health policy. Health nearby]. 2011. URL: http://www.webcitation.org/6LXy28iOg

NATIONAL INSTITUTE FOR PUBLIC HEALTH AND THE ENVIRONMENT (RIVM): Gezondheid op koers? Volksgezondheid toekomst verkenning 2002 [Health on course? Explore the future of public health 2002] Houten: Bohn Stafleu van Loghum; 2002

NATIONAL INSTITUTE FOR PUBLIC HEALTH AND THE ENVIRONMENT (RIVM). Lokale en nationale Monitor gezondheid. Indicatoren voor de Monitor Volksgezondheid [Local and national health monitoring. Indicators for public health monitoring]. 2005. URL: https://www.monitorgezondheid.nl/volksindicatoren.aspx

NATIONAL INSTITUTE FOR PUBLIC HEALTH AND THE ENVIRONMENT (RIVM). Zorgbalans 2008. De prestaties van de Nederlandse gezondheidszorg [Dutch health care performance report]. Bilthoven: RIVM; 2008.

NATIONAL INSTITUTE FOR PUBLIC HEALTH AND THE ENVIRONMENT (RIVM). Volksgezondheid Toekomst Verkenning [Public health forecast]. Bilthoven, The Netherlands: Nationaal Kompas Volksgezondheid; 2012.

NETHERLANDS INSTITUTE FOR HEALTH PROMOTION AND DISEASE PREVENTION (NIGZ). Alcohol. Online adviesop-maat. Drinktest.nl [Alcohol. Online personalized advice. Drinktest.nl]. Resultaten uit de ZonMw Preventieprogramma's. Ingrediënten voor publieke gezondheid. The Hague: ZonMw; 2009: 10-11.

NETHERLANDS INSTITUTE FOR SPORT \& PHYSICAL ACTIVITY Nederlands Instituut voor Sport en Bewegen, NISB). Beweegfeiten [Physical activity facts]. 2013. URL: http://cbs.nisb.nl/30minuten/page/181/Beweegfeiten

NETHERLANDS INSTITUTE OF MENTAL HEALTH AND ADDICTION. Alcoholinfo. Advies volwassenen [Alcohol info. Advice for adults]. 2013a. URL: http://www.alcoholinfo.nl/publiek/watisteveel/hoeveel-per-dag/drinkadviesvolwassenen

NETHERLANDS INSTITUTE OF MENTAL HEALTH AND ADDICTION. Nationale Drug Monitor. Jaarbericht 2012 [National drug monitor. Annual report]. Trimbos Instituut, Utrecht/WODC, Den Haag; 2013b.

NEVILLE LM, O'HARA B, MILAT AJ. Computer-tailored dietary behaviour change interventions: a systematic review. Health Educ Res 2009a; 24:699-720. 
NEVILLE LM, O'HARA B, MILAT A. Computer-tailored physical activity behavior change interventions targeting adults: a systematic review. Int J Behav Nutr Phys Act 2009b; 6:30.

NOAR SM, BENAC CN, HARRIS MS. Does tailoring matter? Meta-analytic review of tailored print health behavior change interventions. Psychol Bull 2007; 133:673-693.

NOAR SM, CHABOT M, ZIMMERMAN RS. Applying health behavior theory to multiple behavior change: considerations and approaches. Prev Med 2008; 46:275-280.

NORMAN GJ, ZABINSKI MF, ADAMS MA, ROSENBERG DE, YAROCH AL, ATIENZA AA. A review of eHealth interventions for physical activity and dietary behavior change. Am J Prev Med 2007; 33:336-345.

O

OCKENE JK, KULLER LH, SVENDSEN KH, MEILAHN E. The relationship of smoking cessation to coronary heart disease and lung cancer in the Multiple Risk Factor Intervention Trial (MRFIT). Am J Public Health 1990; 80:954-958

OENEMA A, BRUG J. Feedback strategies to raise awareness of personal dietary intake: results of a randomized controlled trial. Prev Med 2003; 36:429-439.

OENEMA A, BRUG J, DIJKSTRA A, DE WEERDT I, DE VRIES H. Efficacy and use of an Internet-delivered computertailored lifestyle intervention, targeting saturated fat intake, physical activity and smoking cessation: a randomized controlled trial. Ann Behav Med 2008; 35:125-135.

OENEMA A, TAN F, BRUG J. Short-term efficacy of a web-based computer-tailored nutrition intervention: main effects and mediators. Ann Behav Med 2005; 29:54-63.

ONLINE HEALTH SEARCH. Pew Internet \& American Life Project. Washington, DC. 2006. URL: http://www.pewinternet.org/ /media//Files/Reports/2006/PIP_Online_Health_2006.pdf.pdf

OOIJENDIJK WTM, HILDEBRANDT VH, HOPMAN-ROCK M. Bewegen in Nederland 2000-2005 [Physical activity in the Netherlands 2000-2005]. In: Hildebrandt VH, Ooijendijk WTM, Hopman-Rock M. (Red.). Trendrapport Bewegen en gezondheid 2004/2005. Hoofddorp/Leiden: TNO; 2007.

ORLEANS CT. Addressing multiple behavioral health risks in primary care. Broadening the focus of health behavior change research and practice. Am J Prev Med 2004; 27:1-3.

$\mathrm{P}$

PAINTER JE, BORBA CP, HYNES M, MAYS D, GLANZ K. The use of theory in health behavior research from 2000 to 2005: a systematic review. Ann Behav Med 2008; 35:358-362.

PAREKH S, KING D, OWEN N, JAMROZIK K. Spousal concordance and reliability of the 'Prudence Score' as a summary of diet and lifestyle. Aust N Z J Public Health 2009; 33:320-324.

PAREKH S, VANDELANOTTE C, KING D, BOYLE FM. Improving diet, physical activity and other lifestyle behaviours using computer-tailored advice in general practice: a randomised controlled trial. Int J Behav Nutr Phys Act 2012; 9:108.

PEELS DA, BOLMAN C, GOLSTEIJN RH, DE VRIES H, MUDDE AN, VAN STRALEN MM, LECHNER L. Long-term efficacy of a printed or a Web-based tailored physical activity intervention among older adults. Int J Behav Nutr Phys Act 2013; 10:104. 
PERENBOOM R, OUDSHOORN K, HERTEN L, VAN HOEYMANS N, BIJL R. Levensverwachting in goede geestelijke gezondheid: bepaling afkappunten en wegingsfactoren voor de MHI-5 en GHQ-12 [Life expectancy in good mental health: determination of cut-off points and weighting factors of the MHI-5 and GHQ-12]. Leiden: TNO; 2000.

PERK J, DE BACKER G, GOHLKE H, GRAHAM I, REINER Z, VERSCHUREN M, ALBUS C, BENLIAN P, BOYSEN G, CIFKOVA R, DEATON C, EBRAHIM S, FISHER M, GERMANO G, HOBBS R, HOES A, KARADENIZ S, MEZZANI A, PRESCOTT E, RYDEN L, SCHERER M, SYVÄNNE M, SCHOLTE OP REIMER WJ, VRINTS C, WOOD D, ZAMORANO JL, ZANNAD F; EUROPEAN ASSOCIATION FOR CARDIOVASCULAR PREVENTION \& REHABILITATION (EACPR); ESC COMMITTEE FOR PRACTICE GUIDELINES (CPG). European Guidelines on cardiovascular disease prevention in clinical practice (version 2012). The Fifth Joint Task Force of the European Society of Cardiology and Other Societies on Cardiovascular Disease Prevention in Clinical Practice (constituted by representatives of nine societies and by invited experts). Eur Heart J 2012; 33:1635-1701.

PLOTNIKOFF RC, LUBANS DR, COSTIGAN SA, MCCARGAR L. A test of the theory of planned behavior to predict physical activity in an overweight/obese population sample of adolescents from alberta, Canada. Health Educ Behav 2013; 40:415-425.

POEL F, BOLMAN C, REUBSAET A, DE VRIES H. Efficacy of a single computer tailored mail for smoking cessation: results after six months. Health Educ Res 2009; 24:930-940.

POORTINGA W. The prevalence and clustering of four major lifestyle risk factors in an English adult population. Prev Med 2007; 44:124-128.

PORTNOY DB, SCOTT-SHELDON LA, JOHNSON BT, CAREY MP. Computer-delivered interventions for health promotion and behavioral risk reduction: a meta-analysis of 75 randomized controlled trials, 1988 - 2007. Prev Med 2008; 47:3-16.

POSTEL MG, DE JONG CA, DE HAAN HA. Does e-therapy for problem drinking reach hidden populations? Am J Psychiatry 2005; 162:2393.

POSTEL MG, DE HAAN HA, TER HUURNE ED, BECKER ES, DE JONG CA. Characteristics of problem drinkers in etherapy versus face-to-face treatment. Am J Drug Alcohol Abuse 2011a; 37:537-542.

POSTEL MG, DE HAAN HA, TER HUURNE ED, BECKER ES, DE JONG CA. Effectiveness of a web-based intervention for problem drinkers and reasons for dropout: randomized controlled trial. J Med Internet Res 2010; 12:e68.

POSTEL MG, DE HAAN HA, TER HUURNE ED, VAN DER PALEN J, BECKER ES, DE JONG CA. Attrition in web-based treatment for problem drinkers. J Med Internet Res 2011b; 13:e117.

POULIN C, WEBSTER I, SINGLE E. Alcohol disorders in Canada as indicated by the CAGE questionnaire. CMAJ 1997; 157:1529-1535

POWELL J, HAMBORG T, STALLARD N, BURLS A, MCSORLEY J, BENNETT K, GRIFFITHS KM, CHRISTENSEN H. Effectiveness of a web-based cognitive-behavioral tool to improve mental well-being in the general population: randomized controlled trial. J Med Internet Res 2012; 15:e2.

PRINS RG, VAN EMPELEN P, BEENACKERS MA, BRUG J, OENEMA A. Systematic Development of the YouRAction program, a computer-tailored physical activity promotion intervention for Dutch adolescents, targeting personal motivations and environmental opportunities. BMC Public Health 2010; 10:474.

PROCHASKA JO. Multiple Health Behavior Research represents the future of preventive medicine. Prev Med 2008; 46:281-285. 
PROCHASKA JO, DICLEMENTE CC. Stages and processes of self-change in smoking: toward an integrative model of change. J Consult Clin Psychol 1983; 51:390-395.

PROCHASKA JO, EVERS KE, CASTLE PH, JOHNSON JL, PROCHASKA JM, RULA EY, COBERLEY C, POPE JE. Enhancing multiple domains of well-being by decreasing multiple health risk behaviors: a randomized clinical trial. Popul Health Manag 2012; 15:276-286.

PROCHASKA JJ, NIGG CR, SPRING B, VELICER WF, PROCHASKA JO. The benefits and challenges of multiple health behaviour change in research and in practice. Prev Med 2010; 50:26-29.

PROCHASKA JO, REDDING CA, EVERS KE. The transtheoretical model and stages of change. In: Health Behaviour and Health Education: Theory, Research, and Practice. Third edition. Glanz K (editor). San Francisco: JosseyBass; 1997: 99-120.

PROCHASKA JJ, SPRING B, NIGG CR. Multiple health behavior change research: an introduction and overview. Prev Med 2008; 46:181-188.

PROCHASKA JO, VELICER WF, FAVA JL, ROSSI JS, TSOH JY. Evaluating a population-based recruitment approach and a stage-based expert system intervention for smoking cessation. Addict Behav 2001; 26:583-602.

PROCHASKA JO, VELICER WF, REDDING C, ROSSI JS, GOLDSTEIN M, DEPUE J, GREENE GW, ROSSI SR, SUN X, FAVA JL, LAFORGE R, RAKOWSKI W, PLUMMER BA. Stage-based expert systems to guide a population of primary care patients to quit smoking, eat healthier, prevent skin cancer, and receive regular mammograms. Prev Med 2005; 41:406-416.

PRONK NP, ANDERSON LH, CRAIN AL, MARTINSON BC, O'CONNOR PJ, SHERWOOD NE, WHITEBIRD RR. Meeting recommendations for multiple healthy lifestyle factors; Prevalence, clustering, and predictors among adolescent, adult, and senior health plan members. Am J Prev Med 2004a; 27:25-33.

PRONK NP, PEEK CJ, GOLDSTEIN MG. Addressing multiple behavioral risk factors in primary care. A synthesis of current knowledge and stakeholder dialogue sessions. Am J Prev Med 2004b; 27:4-17.

\section{$\mathrm{R}$}

RAPPANGE DR, BROUWER WB, HOOGENVEEN RT, VAN BAAL PH. Healthcare costs and obesity prevention: drug costs and other sector-specific consequences. Pharmacoeconomics 2009; 27:1031-1044.

REHM J, GREENFIELD TK, ROGERS JD. Average volume of alcohol consumption, patterns of drinking, and all-cause mortality: results from the US National Alcohol Survey. Am J Epidemiol 2001; 153:64-71.

REHM J, MATHERS C, POPOVA S, THAVORNCHAROENSAP M, TEERAWATTANANON Y, PATRA J. Global burden of disease and injury and economic cost attributable to alcohol use and alcohol-use disorders. Lancet 2009; 373:2223-2233.

REHM J, ROOM R, GRAHAM K, MONTEIRO M, GMEL G, SEMPOS CT. The relationship of average volume of alcohol consumption and patterns of drinking to burden of disease: an overview. Addiction 2003; 98:12091228.

REGIONAL PUBLIC HEALTH COMPASS (REGIONAAL KOMPAS VOLKSGEZONDHEID GGD). 2005. URL: http://www.regionaalkompas.nl 
RESNICOW K, DAVIS RE, ZHANG G, KONKEL J, STRECHER VJ, SHAIKH AR. Tailoring a fruit and vegetable intervention on novel motivational constructs: results of a randomized study. Annals of Behavioral Medicine 2008; 35:159-169.

RHODES RE, DE BRUIJN GJ. How big is the physical activity intention-behaviour gap? A meta-analysis using the action control framework. Br J Health Psychol 2013; 18:296-309.

RICHARDSON CR, BUIS LR, JANNEY AW, GOODRICH DE, SEN A, HESS ML, MEHARI KS, FORTLAGE LA, RESNICK PJ, ZIKMUND-FISHER BJ, STRECHER VJ, PIETTE JD. An online community improves adherence in an internetmediated walking program. Part 1: results of a randomized controlled trial. J Med Internet Res. 2010; $12:$ e71.

RIPER H, KRAMER J, SMIT F, CONIJN B, SCHIPPERS G, CUIJPERS P. Web-based self-help for problem drinkers: a pragmatic randomized trial. Addiction 2008; 103:218-227.

RIPER H, VAN STRATEN A, KEUKEN M, SMIT F, SCHIPPERS G, CUIJPERS P. Curbing problem drinking with personalized-feedback interventions: a meta-analysis. Am J Prev Med 2009; 36:247-255.

RITTERBAND LM, GONDER-FREDERICK LA, COX DJ, CLIFTON AD, WEST RW, BOROWITZ S. Internet interventions: in review, in use and into the future. Prof Psychol Res Pr 2003; 34:527-534.

RITTERBAND LM, THORNDIKE FP, COX DJ, KOVATCHEV BP, GONDER-FREDERICK LA. A Behavior Change Model for Internet Interventions. Ann Behav Med 2009; 38:18-27.

ROBROEK SJW, BROUWER W, LINDEBOOM D, OENEMA A, BURDORF A. Demographic, Behavioral, and Psychosocial Correlates of Using the Website Component of a Worksite Physical Activity and Healthy Nutrition Promotion Program: A Longitudinal Study. J Med Internet Res 2010; 12:e44.

RODDA S, LUBMAN DI, DOWLING NA, BOUGH A, JACKSON AC. Web-based counseling for problem gambling: exploring motivations and recommendations. J Med Internet Res 2013; 15:e99.

RODONDI N, HUMAIR JP, GHALI WA, RUFFIEUX C, STOIANOV R, SEEMATTER-BAGNOUD L, STALDER H, PECOUD A, CORNUZ J. Counselling overweight and obese patients in primary care: a prospective cohort study. Eur J Cardiovasc Prev Rehabil 2006; 13:222-228.

ROGERS E. Diffusion of Innovations. New York, NY: The free press; 1995.

ROSNOW RL, ROSENTHAL R. Statistical Procedures and the Justification of Knowledge in Psychological Science. Am Psychol 1989; 44:1276-1284.

RUITER RA, KESSELS LT, JANSMA BM, BRUG J. Increased attention for computer-tailored health communications: an event-related potential study. Health Psychol 2006; 25:300-306.

RUST P, LEHNER P, ELMADFA I. Relationship between dietary intake, antioxidant status and smoking habits in female Austrian smokers. Eur J Nutr 2001; 40:78-83.

RUTTEN GM, SAVELBERG HH, BIDDLE SJ, KREMERS SP. Interrupting long periods of sitting: good STUFF. Int J Behav Nutr Phys Act 2013; 10:1.

RYAN P, LAUVER DR. The efficacy of tailored interventions. J Nurs Scholarsh 2002; 34:331-337. 
SAUNDERS JB, AASLAND OG, BABOR TF, DE LA FUENTE JR, GRANT M. Development of the Alcohol Use Disorders Identification Test (AUDIT): WHO Collaborative Project on Early Detection of Persons with Harmful Alcohol Consumption--II. Addiction 1993; 88:791-804.

SAVELKOUL M, VERWEIJ A, ZANTINGE EM. Zijn er verschillen in alcoholgebruik naar sociaaleconomische status? In: Volksgezondheid Toekomst Verkenning, Nationaal Kompas Volksgezondheid. Bilthoven: RIVM; 2011. URL: http://www.nationaalkompas.nl/gezondheidsdeterminanten/leefstijl/alcoholgebruik/

SCHAFER JL. Multiple imputation: a primer. Stat Methods Med Res 1999; 8:3-15.

SCHNEIDER F, DE VRIES H, CANDEL M, VAN DE KAR A, VAN OSCH L. Periodic email prompts to re-use an internetdelivered computer-tailored lifestyle program: influence of prompt content and timing. J Med Internet Res 2013a; 15:e23.

SCHNEIDER F, SCHULZ DN, POUWELS LH, DE VRIES H, VAN OSCH L. The use of a proactive dissemination strategy to optimize reach of an internet-delivered computer tailored lifestyle intervention. BMC Public Health 2013b; $13: 721$.

SCHNEIDER F, VAN OSCH LA, KREMERS SP, SCHULZ DN, VAN ADRICHEM MJ, DE VRIES H. Optimizing diffusion of an online computer tailored lifestyle program: a study protocol. BMC Public Health 2011; 11:480.

SCHNEIDER F, VAN OSCH L, SCHULZ DN, KREMERS SP, DE VRIES H. The influence of user characteristics and a periodic email prompt on exposure to an internet-delivered computer-tailored lifestyle program. J Med Internet Res 2012; 14:e40.

SCHUIT AJ, VAN LOON JM, TIJHUIS M, OCKÉ MC. Clustering of lifestyle risk factors in a general adult population. Prev Med 2002; 35:219-224.

SCHULZ DN, KREMERS SP, VANDELANOTTE C, VAN ADRICHEM MJG, SCHNEIDER F, CANDEL MJJM, DE VRIES H. Effects of a web-based tailored lifestyle intervention for adults: A two-year randomized controlled trial comparing sequential and simultaneous delivery modes. J Med Internet Res 2014; 16:e26.

SCHULZ DN, KREMERS SP, VAN OSCH LA, SCHNEIDER F, VAN ADRICHEM MJ, DE VRIES H. Testing a Dutch webbased tailored lifestyle programme among adults: a study protocol. BMC Publ Health 2011; 11:108.

SCHULZ DN, KREMERS SP, DE VRIES H. Are the stages of change relevant for the development and implementation of a web-based tailored alcohol intervention? A cross-sectional study. BMC Public Health 2012a; 12:360.

SCHULZ DN, SCHNEIDER F, DE VRIES H, VAN OSCH LA, VAN NIEROP PW, KREMERS SP. Program completion of a web-based tailored lifestyle intervention for adults: differences between a sequential and a simultaneous approach. J Med Internet Res 2012b; 14:e26.

SCHWARZER R. Self-regulatory Processes in the Adoption and Maintenance of Health Behaviors. J Health Psychol 1999; 4:115-127.

SCHWARZER R, LIPPKE S, LUSZCZYNSKA A. Mechanisms of health behavior change in persons with chronic illness or disability: the Health Action Process Approach (HAPA). Rehabil Psychol 2011; 56:161-170.

SCULPHER MJ, CLAXTON K, DRUMMOND M, MCCABE C. Whither trial-based economic evaluation for health care decision making? Health Econ 2006; 15:677-687. 
SEGAN CJ, BORLAND R, GREENWOOD KM. What is the right thing at the right time? Interactions between stages and processes of change among smokers who make a quit attempt. Health Psychol 2004; 23:86-93.

SEVERSON HH, GORDON JS, DANAHER BG, AKERS L. ChewFree.com: evaluation of a Web-based cessation program for smokeless tobacco users. Nicotine Tob Res 2008; 10:381-391.

SILAGY C, MUIR J, COULTER A, THOROGOOD M, ROE L. Cardiovascular risk and attitudes to lifestyle: what do patients think? BMJ 1993; 306:1657-1660.

SINHARAY S, STERN HS, RUSSELL D. The use of multiple imputation for the analysis of missing data. Psychol Methods 2001; 6:317-329.

SKINNER CS, CAMPBELL MK, RIMER BK, CURRY S, PROCHASKA JO. How effective is tailored print communication? Ann Behav Med 1999; 21:290-298.

SMEETS T. Towards a healthy lifestyle: the development and evaluation of a computer tailored lifestyle intervention. Dissertation. Maastricht University, Maastricht; 2006.

SMEETS T, BRUG J, DE VRIES H. Effects of tailoring health messages on physical activity. Health Educ Res 2008; 23:402-413.

SMEETS T, KREMERS S, BRUG J, DE VRIES H. Effects of tailored feedback on multiple health behaviors. Ann Behav Med 2007; 33:117-123.

SMIT ES, DE VRIES H, HOVING C. Effectiveness of a Web-based multiple tailored smoking cessation program: a randomized controlled trial among Dutch adult smokers. J Med Internet Res 2012a; 14:e82.

SMIT ES, DE VRIES H, HOVING C. The PAS study: a randomized controlled trial evaluating the effectiveness of a web-based multiple tailored smoking cessation programme and tailored counselling by practice nurses. Contemp Clin Trials 2010; 31:251-258.

SMIT ES, EVERS SM, DE VRIES H, HOVING C. Cost-effectiveness and cost-utility of Internet-based computer tailoring for smoking cessation. J Med Internet Res 2013; 15:e57.

SMIT ES, HOVING C, COX VC, DE VRIES H. Influence of recruitment strategy on the reach and effect of a webbased multiple tailored smoking cessation intervention among Dutch adult smokers. Health Educ Res 2012b; 27:191-199.

SMIT F, LOKKERBOL J, RIPER H, MAJO MC, BOON B, BLANKERS M. Modeling the cost-effectiveness of health care systems for alcohol use disorders: how implementation of eHealth interventions improves cost-effectiveness. J Med Internet Res 2011; 13:e56.

SNIEHOTTA FF, SCHOLZ U, SCHWARZER R. Action plans and coping plans for physical exercise: A longitudinal intervention study in cardiac rehabilitation. Br J Health Psychol. 2006; 11:23-37.

SOBELL LC, CELLUCCI T, NIRENBERG TD, SOBELL MB. Do quantity-frequency data underestimate drinking-related health risks? Am J Public Health 1982; 72:823-828.

SPENCER CA, JAMROZIK K, NORMAN PE, LAWRENCE-BROWN M. A simple lifestyle score predicts survival in healthy elderly men. Prev Med 2005; 40:712-717.

SPITTAELS H, DE BOURDEAUDHUIJ I. Volwassenen tot beweging stimuleren via het Internet [Stimulating adults via the Internet to be physically active]. In: Steens G, editor. Moet er nog sport zijn? Sport, beweging en gezondheid in Vlaanderen. Volume 1. Antwerp, Belgium: F\&G Partners; 2006. 
SPITTAELS H, DE BOURDEAUDHUIJ I, BRUG J, VANDELANOTTE C. Effectiveness of an online computer-tailored physical activity intervention in a real-life setting. Health Educ Res 2007a; 22:385-396.

SPITTAELS H, DE BOURDEAUDHUIJ I, VANDELANOTTE C. Evaluation of a website-delivered computer-tailored intervention for increasing physical activity in the general population. Prev Med 2007b; 44:209-217.

SPRING B, PAGOTO S, PINGITORE R, DORAN N, SCHNEIDER K, HEDEKER D. Randomized controlled trial for behavioral smoking and weight control treatment: effect of concurrent versus sequential intervention. J Consult Clin Psychol 2004; 72:785-796.

STANCZYK NE, BOLMAN C, VAN ADRICHEM MJG, CANDEL MJJM, MURIS JWM, DE VRIES H. Comparison of Text and Video Computer-Tailored Interventions for Smoking Cessation: Randomized Controlled Trial. J Med Internet Res 2014; 16:e69.

STANCZYK NE, BOLMAN C, MURIS JW, DE VRIES H. Study protocol of a Dutch smoking cessation e-health program. BMC Public Health 2011; 11:847.

STANCZYK NE, BOLMAN C, SMIT ES, CANDEL MJ, MURIS JW, DE VRIES H. How to encourage smokers to participate in web-based computer-tailored smoking cessation programs: a comparison of different recruitment strategies. Health Educ Res 2013.

STANCZYK NE, DE VRIES H, CANDEL MJJM, MURIS JWM, BOLMAN C. Effectiveness of a video-and text-based computer-tailored smoking cessation intervention among LSES and HSES smokers. Submitted a.

STANCZYK NE, SMIT ES, SCHULZ DN, BOLMAN C, MURIS J, DE VRIES H, EVERS SMAA. Examining the costeffectiveness and cost-utility of a video-based and text-based computer-tailored lifestyle intervention for smoking cessation. Submitted b.

STATISTICS NETHERLANDS (Centraal Bureau voor de Statistiek, CBS). Persbericht Leefstijl Nederlanders niet verbeterd [Press release Lifestyle Dutch people not improved]. 2007a. URL: http://www.cbs.nl/NR/rdonlyres/EFCD6D79-C4A4-42C4-BCA9-D8EDFE67039E/0/pb07n021.pdf

STATISTICS NETHERLANDS (Centraal Bureau voor de Statistiek, CBS). Permanent Onderzoek Leefsituatie (POLS), gezondheid en welzijn. 2007b.

STATISTICS NETHERLANDS (Centraal Bureau voor de Statistiek, CBS). Permanent Onderzoek Leefsituatie (POLS), gezondheid en welzijn. 2009.

STATISTICS NETHERLANDS (Centraal Bureau voor de Statistiek, CBS). ICT gebruik van huishoudens naar huishoudkenmerken [ICT use of households by household characteristics]. Den Haag/Heerlen; 2011 URL: http://statline.cbs.nl/statweb/

STATISTICS NETHERLANDS (Centraal Bureau voor de Statistiek, CBS). Onderzoek ICT gebruik van huishoudens [Survey ICT use by households]. Den Haag/Heerlen; 2012. URL: http://statline.cbs.nl/statweb/

STATISTICS NETHERLANDS (Centraal Bureau voor de Statistiek, CBS). StatLine. ICT gebruik van huishoudens naar huishoudkenmerken 2005-2013 [ICT use of households by household characteristics 2005-2013]. Den Haag/Heerlen; 2013a. URL: http://statline.cbs.nl/statweb/

STATISTICS NETHERLANDS (Centraal Bureau voor de Statistiek, CBS). StatLine. Leefstijl, preventief onderzoek; persoonskenmerken 2010-2012 [Lifestyle, preventive research: personal characteristics 2010-2012]. Den Haag/Heerlen; 2013b. URL: http://statline.cbs.nl/statweb/ 
STATISTICS NETHERLANDS (Centraal Bureau voor de Statistiek, CBS). StatLine. Consumentenprijzen; prijsindex 2006 = 100. Den Haag/Heerlen; 2013c. URL: http://statline.cbs.nl/statweb/

STINNETT AA, MULLAHY J. Net health benefits: a new framework for the analysis of uncertainty in costeffectiveness analysis. Med Decis Making 1998; 18:68-80.

STIVORO. Roken in Nederland toegenomen 2012 [Smoking rates increased in the Netherlands 2012]. Den Haag; 2013. URL: http://stivoro.nl/2013/02/21/roken-in-nederland-toegenomen/

STOCK C, MIKOLAJCZYK R, BLOOMFIELD K, MAXWELL AE, OZCEBE H, PETKEVICIENE J, NAYDENOVA V, MARINFERNANDEZ B, EL-ANSARI W, KRÄMER A. Alcohol consumption and attitudes towards banning alcohol sales on campus among European university students. Public Health 2009; 123:122-129.

STODDARD JL, AUGUSTSON EM, MOSER RP. Effect of adding a virtual community (bulletin board) to smokefree.gov: randomized controlled trial. J Med Internet Res 2008; 10:e53.

STRECHER VJ, MCCLURE J, ALEXANDER G, CHAKRABORTY B, NAIR V, KONKEL J, GREENE S, COUPER M, CARLIER C, WIESE C, LITTLE R, POMERLEAU C, POMERLEAU O. The role of engagement in a tailored web-based smoking cessation program: randomized controlled trial. J Med Internet Res 2008; 10:e36.

STRECHER V, WANG C, DERRY H, WILDENHAUS K, JOHNSON C. Tailored interventions for multiple risk behaviors. Health Educ Res 2002; 17:619-626.

SUTTON S. Can "stages of change" provide guidance in the treatment of addictions? A critical examination of Prochaska and DiClemente's model. In: Edwards G, Dare C (editors). Psychotherapy, Psychological Treatments and the Addictions. Cambridge: Cambridge University Press; 1996; 189-205.

\section{T}

TAMAKOSHI A, TAMAKOSHI K, LIN Y, YAGYU K, KIKUCHI S; JACC STUDY GROUP. Healthy lifestyle and preventable death: findings from the Japan Collaborative Cohort (JACC) Study. Prev Med 2009; 48:486-492.

TATE DF, FINKELSTEIN EA, KHAVJOU O, GUSTAFSON A. Cost effectiveness of internet interventions: review and recommendations. Ann Behav Med 2009; 38:40-45.

TATE DF, WING RR, WINETT RA. Using Internet technology to deliver a behavioral weight loss program. JAMA $2001 ; 285: 1172-1177$

TAYLOR WC, HEPWORTH JT, LEES E, CASSELLS A, GOUSSE Y, SWEENEY MM, VAUGHN A, TOBIN JN. Readiness to change physical activity and dietary practices and willingness to consult healthcare providers. Health Res Pol Sys $2004 ; 2: 2$

TE POEL F, BOLMAN C, REUBSAET A, DE VRIES H. Efficacy of a single computer-tailored e-mail for smoking cessation: results after 6 months. Health Educ Res 2009; 24:930-940.

THE DUTCH INSTITUTE FOR HEALTHCARE IMPROVEMENT (Kwaliteitsinstituut voor de Gezondheidszorg CBO). Richtlijn behandeling van tabaksverslaving [Guidelines for treating tobacco addiction]. Utrecht/The Hague: BVO/Partnership Stop met Roken; 2004.

THE HEALTH COUNCIL OF THE NETHERLANDS (Gezondheidsraad). Richtlijnen goede voeding 2006 achtergronddocument [Guidelines for a healthy diet 2006 - background material]. The Hague: Gezondheidsraad; 2006. 
TILLMANN HC, SHARPE N, SPONER G, WEHLING M. Does intention-to-treat analysis answer all questions in longterm mortality trials? Considerations on the basis of the ANZ trial. Int J Clin Pharmacol Ther 2001; 39:205212.

TNS OPINION \& SOCIAL, 2012. Media use in the European Union. Report. Autumn 2011. Standard Eurobarometer 76. URL: http://ec.europa.eu/public_opinion/archives/eb/eb76/eb76_media_en.pdf

TUCKER M, REICKS M. Exercise as a gateway behavior for healthful eating among older adults: an exploratory study. J Nutr Educ Behav 2002; 34:14-19.

\section{U}

UITERS E, VERWEIJ A. Lichamelijke activiteit: Zijn er verschillen naar sociaaleconomische status? In: Volksgezondheid Toekomst Verkenning, Nationaal Kompas Volksgezondheid. Bilthoven: RIVM; 2013. URL: http://www.nationaalkompas.nl/gezondheidsdeterminanten/leefstijl/lichamelijke-activiteit/

UNITED STATES DEPARTMENT OF HEALTH AND HUMAN SERVICES (USDHHS), 2000. Healthy People 2010. Conference Edition. U.S. Department of Health and Human Services, Washington, D.C.

\section{V}

VAN ASSEMA P, BRUG J, RONDA G, STEENHUIS I, OENEMA A. A short dutch questionnaire to measure fruit and vegetable intake: relative validity among adults and adolescents. Nutr Health 2002; 16:85-106.

VANDELANOTTE C. Are simultaneously delivered health behaviour change interventions the way of the future?: a comment on King et al. Ann Behav Med 2013; 46:133-134.

VANDELANOTTE C, CAPERCHIONE CM, ELLISON M, GEORGE ES, MAEDER A, KOLT GS, DUNCAN MJ, KARUNANITHI M, NOAKES M, HOOKER C, VILJOEN P, MUMMERY WK. What kinds of website and mobile phone-delivered physical activity and nutrition interventions do middle-aged men want? J Health Commun 2013; 18:1070-1083.

VANDELANOTTE C, DE BOURDEAUDHUIJ I. Acceptability and feasibility of a computer-tailored physical activity intervention using stages of change: project FAITH. Health Educ Res 2003; 18:304-317.

VANDELANOTTE C, DE BOURDEAUDHUIJ I, BRUG J. Acceptability and feasibility of an interactive computertailored fat intake intervention in Belgium. Health Promot Int 2004; 19:463-470.

VANDELANOTTE C, DE BOURDEAUDHUIJ I, BRUG J. Two-year follow-up of sequential and simultaneous interactive computer-tailored interventions for increasing physical activity and decreasing fat intake. Ann Behav Med 2007a; 33:213-219.

VANDELANOTTE C, DE BOURDEAUDHUIJ I, SALLIS JF, SPITTAELS H, BRUG J. Efficacy of sequential or simultaneous interactive computer-tailored interventions for increasing physical activity and decreasing fat intake. Ann Behav Med 2005; 29:138-146.

VANDELANOTTE C, MUMMERY WK. Qualitative and quantitative research into the development and feasibility of a video-tailored physical activity intervention. Int J Behav Nutr Phys Act 2011; 8:70.

VANDELANOTTE C, REEVES MM, BRUG J, DE BOURDEAUDHUIJ I. A randomized trial of sequential and simultaneous multiple behavior change interventions for physical activity and fat intake. Prev Med 2008; 46:232-237. 
VANDELANOTTE C, SPATHONIS KM, EAKIN EG, OWEN N. Website-Delivered Physical Activity Interventions. A Review of the Literature. Am J Prev Med 2007b; 33:54-64.

VAN DEN BERG MH, SCHOONES JW, VLIET VLIELAND TP. Internet-based physical activity interventions: a systematic review of the literature. J Med Internet Res 2007; 9:e26.

VAN GENUGTEN L, VAN EMPELEN P, BOON B, BORSBOOM G, VISSCHER T, OENEMA A. Results from an online computer-tailored weight management intervention for overweight adults: randomized controlled trial. J Med Internet Res 2012; 14:e44.

VAN KEULEN HM, BOSMANS JE, VAN TULDER MW, SEVERENS JL, DE VRIES H, BRUG J, MESTERS I. Costeffectiveness of tailored print communication, telephone motivational interviewing, and a combination of the two: results of an economic evaluation alongside the Vitalum randomized controlled trial. Int J Behav Nutr Phys Act 2010a; 7:64.

VAN KEULEN HM, MESTERS I, AUSEMS M, VAN BREUKELEN G, CAMPBELL M, RESNICOW K, BRUG J, DE VRIES H. Tailored print communication and telephone motivational interviewing are equally successful in improving multiple lifestyle behaviors in a randomized controlled trial. Ann Behav Med 2011; 41:104-118.

VAN KEULEN HM, MESTERS I, VAN MECHELEN W, DE VRIES H. Single-item and multiple-item measures of adherence to public health behavior guidelines were incongruent. J Clin Epidemiol 2010b; 63:75-84.

VANKIPURAM M, MCMAHON S, FLEURY J. ReadySteady: app for accelerometer-based activity monitoring and wellness-motivation feedback system for older adults. AMIA Annu Symp Proc 2012; 2012:931-939.

VAN OERS JA, BONGERS IM, VAN DE GOOR LA, GARRETSEN HF. Alcohol consumption, alcohol-related problems, problem drinking, and socioeconomic status. Alcohol Alcohol 1999; 34:78-88.

VAN OORT FV, VAN LENTHE FJ, MACKENBACH JP. Cooccurrence of lifestyle risk factors and the explanation of education inequalities in mortality: results from the GLOBE study. Prev Med 2004; 39:1126-1134.

VAN OSCH L, BEENACKERS M, REUBSAET A, LECHNER L, CANDEL M, DE VRIES H. Action planning as predictor of health protective and health risk behavior: an investigation of fruit and snack consumption. Int J Behav Nutr Phys Act 2009; 6:69.

VAN OSCH L, LECHNER L, REUBSAET A, DE VRIES H. From theory to practice. An explorative study into the instrumentality and specificity of implementation intentions. Psychol Health 2010; 25:351-364.

VAN OSCH L, REUBSAET A, LECHNER L, DE VRIES $H$. The formation of specific action plans can enhance sun protection behaviour in motivated parents. Prev Med 2008; 47:127-132.

VAN ROSSUM CTM, FRANSEN HP, VERKAIK-KLOOSTERMAN J, BUURMA-RETHANS EJM, OCKÉ MC. Dutch National Food Consumption Survey 2007-2010: Diet of children and adults aged 7 to 69 years. RIVM-rapport nr. 350050006. Bilthoven; 2011.

VAN STRALEN MM, DE VRIES H, MUDDE AN, BOLMAN C, LECHNER L. Efficacy of two tailored interventions promoting physical activity in older adults. Am J Prev Med 2009a; 37:405-417.

VAN STRALEN MM, DE VRIES H, MUDDE AN, BOLMAN C, LECHNER L. The long-term efficacy of two computertailored physical activity interventions for older adults: main effects and mediators. Health Psychol 2011; 30:442-452. 
VAN STRALEN MM, DE VRIES H, MUDDE AN, BOLMAN C, LECHNER L. The working mechanisms of an environmentally tailored physical activity intervention for older adults: a randomized controlled trial. Int J Behav Nutr Phys Act 2009b; 6:83.

VAN 'T RIET J, CRUTZEN R, DE VRIES H. Investigating predictors of visiting, using, and revisiting an online healthcommunication program: a longitudinal study. J Med Internet Res 2010; 12:e37.

VAN WIER MF, DEKKERS JC, BOSMANS JE, HEYMANS MW, HENDRIKSEN IJ, PRONK NP, VAN MECHELEN W, VAN TULDER MW. Economic evaluation of a weight control program with e-mail and telephone counseling among overweight employees: a randomized controlled trial. Int J Behav Nutr Phys Act 2012; 9:112.

VELICER WF, NORMAN GJ, FAVA JL, PROCHASKA JO. Testing 40 predictions from the transtheoretical model. Addict Behav 1999a; 24:455-469.

VELICER WF, PROCHASKA JO, FAVA JL, LAFORGE RG, ROSSI JS. Interactive versus noninteractive interventions and dose - response relationships for stage-matched smoking cessation programs in a managed care setting. Health Psychol 1999b; 18:21-28.

VELICER WF, REDDING CA, PAIVA AL, MAURIELLO LM, BLISSMER B, OATLEY K, MEIER KS, BABBIN SF, MCGEE H, PROCHASKA JO, BURDITT C, FERNANDEZ AC. Multiple behavior interventions to prevent substance abuse and increase energy balance behaviors in middle school students. Transl Behav Med 2013; 3:82-93.

VERHEIJDEN MW, JANS MP, HILDEBRANDT VH. Web-based tailored lifestyle programs: exploration of the target group's interests and implications for practice. Health Promot Pract 2008; 9:82-92.

VERHEIJDEN MW, JANS MP, HILDEBRANDT VH, HOPMAN-ROCK M. Rates and determinants of repeated participation in a web-based behavior change program for healthy body weight and healthy lifestyle. J Med Internet Res 2007; 9:e1.

VERPLANKEN B, ORBELL S. Reflections on past behavior: a self-report index of habit strength. J Appl Soc Psychol 2003; 33:1313-1330.

VITÓRIA PD, SALGUEIRO MF, SILVA SA, DE VRIES H. The impact of social influence on adolescent intention to smoke: combining types and referents of influence. Br J Health Psychol 2009; 14:681-699.

VON LENGERKE T, KRAUTH C. Economic costs of adult obesity: a review of recent European studies with a focus on subgroup-specific costs. Maturitas 2011; 69:220-229.

VOOGT CV, POELEN EA, KLEINJAN M, LEMMERS LA, ENGELS RC. The development of a web-based brief alcohol intervention in reducing heavy drinking among college students: an Intervention Mapping approach. Health Promot Int 2013; 22.

\section{W}

WALLACE P, MURRAY E, MCCAMBRIDGE J, KHADJESARI Z, WHITE IR, THOMPSON SG, KALAITZAKI E, GODFREY C, LINKE S. On-line randomized controlled trial of an internet based psychologically enhanced intervention for people with hazardous alcohol consumption. PLoS One 2011; 6:e14740.

WALTER S, MACKENBACH J, VOKÓ Z, LHACHIMI S, IKRAM MA, UITTERLINDEN AG, NEWMAN AB, MURABITO JM, GARCIA ME, GUDNASON V, TANAKA T, TRANAH GJ, WALLASCHOFSKI H, KOCHER T, LAUNER LJ, FRANCESCHINI N, SCHIPPER M, HOFMAN A, TIEMEIER H. Genetic, physiological, and lifestyle predictors of mortality in the general population. Am J Public Health 2012; 102:e3-10. 
WALTHOUWER MJ, OENEMA A, SOETENS K, LECHNER L, DE VRIES H. Systematic development of a text-driven and a video-driven web-based computer-tailored obesity prevention intervention. BMC Public Health 2013; 13:978.

WANTLAND DJ, PORTILLO CJ, HOLZEMER WL, SLAUGHTER R, MCGHEE EM. The effectiveness of Web-based vs. non-Web-based interventions: a meta-analysis of behavioral change outcomes. J Med Internet Res 2004; $6: e 40$.

WARE JE, KOSINSKI M, KELLER SD. SF-12. how to score the SF-12 physical and mental health summary scales Boston: The Health Institute, New England Medical Center; 1995.

WARE JE, KOSINSKI M, KELLER SD. A 12-item Short-Form health survey: construction of scales and preliminary tests of reliability and validity. Med Care 1996; 34:220-233.

WARMERDAM L, SMIT F, VAN STRATEN A, RIPER H, CUIJPERS P. Cost-utility and cost-effectiveness of internetbased treatment for adults with depressive symptoms: randomized trial. J Med Internet Res 2010; 12 : e53.

WEBB TL, JOSEPH J, YARDLEY L, MICHIE S. Using the internet to promote health behavior change: a systematic review and meta-analysis of the impact of theoretical basis, use of behavior change techniques, and mode of delivery on efficacy. J Med Internet Res 2010; 12:e4.

WEINSTEIN MC, STASON WB. Foundations of cost-effectiveness analysis for health and medical practices. N EngI J Med 1977; 296:716-721.

WENDEL-VOS GCW. Hoeveel mensen zijn voldoende lichamelijk actief? In: Volksgezondheid Toekomst Verkenning, Nationaal Kompas Volksgezondheid. Bilthoven: RIVM; $2013 . \quad$ URL: http://www.nationaalkompas.nl/gezondheidsdeterminanten/leefstij//lichamelijke-activiteit/

WENDEL-VOS G, SCHUIT A, SARIS W, KROMHOUT D. Reproducibility and relative validity of the short questionnaire to assess health enhancing physical activity. J Clin Epidemiol 2003; 56:1163-1169.

WEST R. Time for a change: Putting the transtheoretical (stages of change) model to rest. Addiction 2005; 100:1036-1039.

WEST R. The clinical significance of "small" effects of smoking cessation treatments. Addiction 2007; 102:506509.

WETZELS JJ, KREMERS SP, VITÓRIA PD, DE VRIES H. The alcohol-tobacco relationship: a prospective study among adolescents in six European countries. Addiction 2003; 98:1755-1763.

WHITE A, KAVANAGH D, STALLMA H, KLEIN B, KAY-LAMBKIN F, PROUDFOOT J, DRENNAN J, CONNOR J, BAKER A, HINES E, YOUNG R. Online alcohol interventions: a systematic review. J Med Internet Res 2010; 12:e62.

WHITE IR, ROYSTON P, WOOD AM. Multiple imputation using chained equations: Issues and guidance for practice. Stat Med 2011; 30:377-399.

WIGGERS LC, OORT FJ, PETERS RJ, LEGEMATE DA, DE HAES HC, SMETS EM. Smoking cessation may not improve quality of life in atherosclerotic patients. Nicotine Tob Res 2006; 8:581-9.

WILJER D, UROWITZ S, BARBERA L, CHIVERS ML, QUARTEY NK, FERGUSON SE, TO M, CLASSEN CC. A qualitative study of an internet-based support group for women with sexual distress due to gynecologic cancer. J Cancer Educ 2011; 26:451-458. 
WOOLF SH, KRIST AH, JOHNSON RE, WILSON DB, ROTHEMICH SF, NORMAN GJ, DEVERS KJ. A practice-sponsored Web site to help patients pursue healthy behaviors: an ACORN study. Ann Fam Med 2006; 4:148-152.

WORLD HEALTH ORGANIZATION. The World Health Report 2002. Reducing risks, promoting healthy life. WHO; 2002.

WORLD HEALTH ORGANIZATION. Global alcohol report 1990-2006. WHO; $2006 . \quad$ URL: http://www.who.int/substance_abuse/publications/global_alcohol_report/msbgsreur.pdf

WORLD HEALTH ORGANIZATION. The top ten causes of death. WHO fact sheet Geneva: WHO; 2007.

WORLD HEALTH ORGANIZATION. WHO fact sheet. Geneva: WHO; 2011.

\section{Y}

YANG S, LYNCH J, SCHULENBERG J, ROUX AV, RAGHUNATHAN T. Emergence of socioeconomic inequalities in smoking and overweight and obesity in early adulthood: the national longitudinal study of adolescent health. Am J Public Health 2008; 98:468-477.

\section{Z}

ZANTINGE EM, UITERS E. Chronische ziekten en multimorbiditeit: Zijn er verschillen naar sociaaleconomische status? In: Volksgezondheid Toekomst Verkenning, Nationaal Kompas Volksgezondheid. Bilthoven: RIVM; 2009. URL: http://www.nationaalkompas.nl/gezondheid-en-ziekte/ziekten-en-aandoeningen/chronischeziekten-en-multimorbiditeit/

ZUCKERMAN M, PORAC J, LATHIN D, SMITH R, DECI EL. On the importance of self-determination for intrinsically motivated behaviour. Pers Soc Psychol Bull 1978; 4:443-446. 



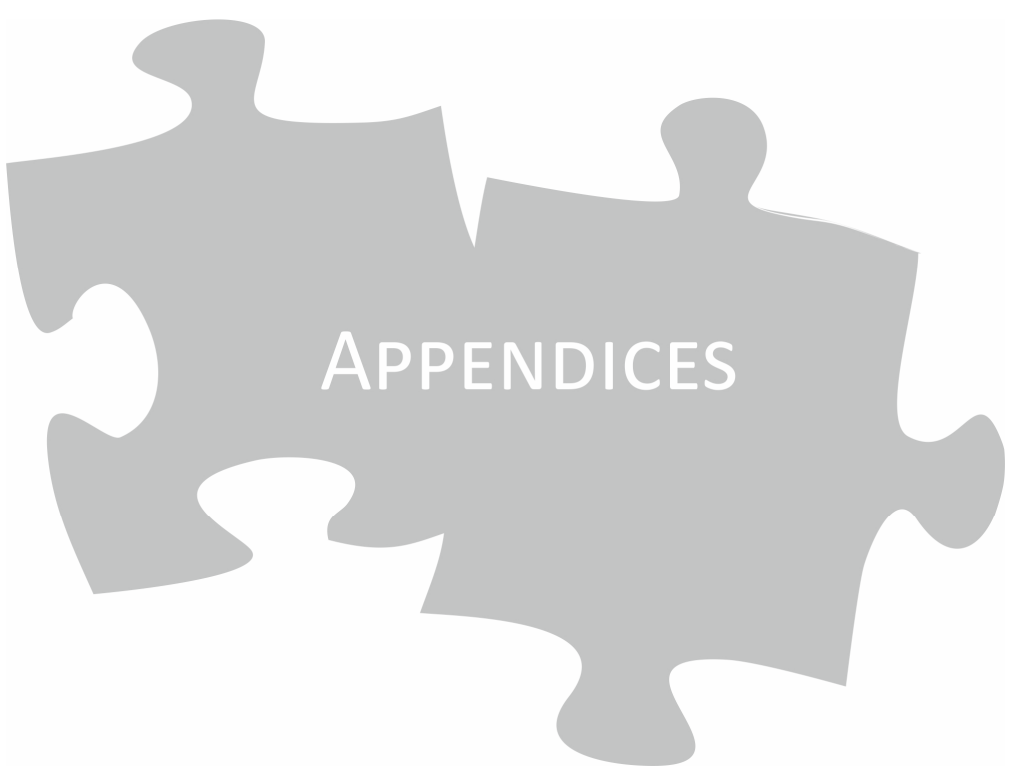





\section{APPENDIX 1}

Table I Results of the logistic regression analysis (backward method) with guideline status (not complying = 0; complying $=1$ ) after six months as dependent variable and results of the linear regression analysis (backward method) with the number of alcoholic drinks after six months as dependent variable after filling missing values with the last observation (LOCF; $n=321$ )

\begin{tabular}{|c|c|c|c|c|c|c|}
\hline \multirow[b]{2}{*}{ Variable a } & \multicolumn{3}{|c|}{ Guideline status (LOCF) } & \multicolumn{3}{|c|}{ Number of drinks (LOCF) } \\
\hline & OR & $p$ & $95 \% \mathrm{Cl}$ & $\beta$ & $p$ & $95 \% \mathrm{Cl}$ \\
\hline Condition & 2.29 & .009 & $1.23-4.26$ & -.07 & .09 & $-4.80-0.32$ \\
\hline Guideline status & 4.88 & $<.001$ & $2.32-10.27$ & --- & --- & --- \\
\hline Weekly alcohol intake & 0.93 & .002 & $0.89-0.97$ & .58 & $<.001$ & $0.61-0.84$ \\
\hline Habit & 0.34 & $<.001$ & $0.22-0.53$ & .14 & .006 & $0.71-4.32$ \\
\hline Age & 0.98 & .08 & $0.96-1.00$ & --- & -- & --- \\
\hline Social norm & --- & --- & --- & -.08 & .07 & $-2.04-0.09$ \\
\hline Self-efficacy & 0.48 & .003 & $0.29-0.77$ & .10 & .045 & $0.05-3.97$ \\
\hline$R^{2}$ & .49 & & & .43 & & \\
\hline
\end{tabular}

Note: ${ }^{a}$ Assessed at baseline. 


\section{APPENDIX 2}

Table II Results of the dropout analyses

\begin{tabular}{|c|c|c|c|}
\hline Variable $^{a}$ & $\begin{array}{l}\text { Differences } \\
\text { T1C vs. NC }{ }^{b}\end{array}$ & $\begin{array}{l}\text { Differences } \\
\text { T2C vs. NC }{ }^{b}\end{array}$ & $\begin{array}{l}\text { Differences } \\
\text { FCC vs. NC }{ }^{b}\end{array}$ \\
\hline \multicolumn{4}{|l|}{ DEMOGRAPHICS } \\
\hline Age (19-65) & $\mathrm{T} 1 \mathrm{C}>\mathrm{NC}$ & $\mathrm{T} 2 \mathrm{C}>\mathrm{NC}$ & $\mathrm{FCC}>\mathrm{NC}$ \\
\hline $\begin{array}{l}\text { Employment situation } n=4,970 \\
\text { Job (paid employment) } \\
\text { No job }\end{array}$ & $\mathrm{T} 1 \mathrm{C}<\mathrm{NC}$ & $\mathrm{T} 2 \mathrm{C}<\mathrm{NC}$ & $\mathrm{FCC}<\mathrm{NC}$ \\
\hline $\begin{array}{l}\text { Marital status } n=4,973 \\
\text { Relationship } \\
\text { Single }\end{array}$ & $\mathrm{T} 1 \mathrm{C}>\mathrm{NC}$ & $\mathrm{T} 2 \mathrm{C}>\mathrm{NC}$ & $\mathrm{FCC}>\mathrm{NC}^{+}$ \\
\hline \# persons in household $n=4,980$ & $\mathrm{~T} 1 \mathrm{C}<\mathrm{NC}$ & $\mathrm{T} 2 \mathrm{C}<\mathrm{NC}$ & $\mathrm{FCC}<\mathrm{NC}$ \\
\hline $\begin{array}{l}\text { Native country } n=4,973 \\
\text { The Netherlands } \\
\text { Other }\end{array}$ & $\mathrm{T} 1 \mathrm{C}>\mathrm{NC}$ & & \\
\hline \multicolumn{4}{|l|}{ HEALTH STATUS } \\
\hline BMI $(13.82-58.11) n=5,012$ & & $\mathrm{~T} 2 \mathrm{C}>\mathrm{NC}$ & \\
\hline Psychol. distress (12-50) $n=4,944$ & & & $\mathrm{FCC}>\mathrm{NC}^{+}$ \\
\hline \multicolumn{4}{|l|}{ Disease(s) $n=4,950$} \\
\hline Diabetes & $\mathrm{T} 1 \mathrm{C}>\mathrm{NC}$ & $\mathrm{T} 2 \mathrm{C}>\mathrm{NC}^{\dagger}$ & \\
\hline \multicolumn{4}{|l|}{ Brain haemorrhage, TIA } \\
\hline Heart attack & $\mathrm{T} 1 \mathrm{C}>\mathrm{NC}$ & $\mathrm{T} 2 \mathrm{C}>\mathrm{NC}$ & $\mathrm{FCC}>\mathrm{NC}$ \\
\hline Other serious heart disease & $\mathrm{T} 1 \mathrm{C}>\mathrm{NC}^{+}$ & $\mathrm{T} 2 \mathrm{C}>\mathrm{NC}$ & $\mathrm{FCC}>\mathrm{NC}$ \\
\hline Cancer & $\mathrm{T} 1 \mathrm{C}>\mathrm{NC}^{+}$ & & \\
\hline $\begin{array}{l}\text { High blood pressure } \\
\text { Asthma, COPD }\end{array}$ & $\mathrm{T} 1 \mathrm{C}>\mathrm{NC}$ & $\mathrm{T} 2 \mathrm{C}>\mathrm{NC}$ & $\mathrm{FCC}>\mathrm{NC}$ \\
\hline One or more diseases & $\mathrm{T} 1 \mathrm{C}>\mathrm{NC}$ & $\mathrm{T} 2 \mathrm{C}>\mathrm{NC}$ & $\mathrm{FCC}>\mathrm{NC}$ \\
\hline \multicolumn{4}{|l|}{ LIFESTYLE BEHAVIOUR } \\
\hline Mean number of risk factors & $\mathrm{T} 1 \mathrm{C}<\mathrm{NC}$ & $\mathrm{T} 2 \mathrm{C}<\mathrm{NC}$ & $\mathrm{FCC}<\mathrm{NC}$ \\
\hline \multicolumn{4}{|l|}{ Physical activity $n=5,053$} \\
\hline Mean number of minutes per day & $\mathrm{T} 1 \mathrm{C}>\mathrm{NC}$ & & $\mathrm{FCC}>\mathrm{NC}^{+}$ \\
\hline Non-compliance & $\mathrm{T} 1 \mathrm{C}<\mathrm{NC}$ & & $\mathrm{FCC}<\mathrm{NC}$ \\
\hline \multicolumn{4}{|l|}{ Vegetable consumption $n=5,018$} \\
\hline Mean number of grams & $\mathrm{T} 1 \mathrm{C}>\mathrm{NC}$ & $\mathrm{T} 2 \mathrm{C}>\mathrm{NC}$ & $\mathrm{FCC}>\mathrm{NC}$ \\
\hline Non-compliance & $\mathrm{T} 1 \mathrm{C}<\mathrm{NC}$ & $\mathrm{T} 2 \mathrm{C}<\mathrm{NC}$ & $\mathrm{FCC}<\mathrm{NC}$ \\
\hline \multicolumn{4}{|l|}{ Fruit consumption $n=5,019$} \\
\hline Mean pieces of fruit & $\mathrm{T} 1 \mathrm{C}>\mathrm{NC}$ & $\mathrm{T} 2 \mathrm{C}>\mathrm{NC}$ & $\mathrm{FCC}>\mathrm{NC}$ \\
\hline Non-compliance & $\mathrm{T} 1 \mathrm{C}<\mathrm{NC}$ & $\mathrm{T} 2 \mathrm{C}<\mathrm{NC}$ & $\mathrm{FCC}<\mathrm{NC}$ \\
\hline \multicolumn{4}{|l|}{ Alcohol intake $n=5,034$} \\
\hline Mean number of drinks & & $\mathrm{T} 2 \mathrm{C}<\mathrm{NC}$ & $\mathrm{FCC}<\mathrm{NC}$ \\
\hline Non-compliance & $\mathrm{T} 1 \mathrm{C}<\mathrm{NC}^{\dagger}$ & $\mathrm{T} 2 \mathrm{C}<\mathrm{NC}$ & $\mathrm{FCC}<\mathrm{NC}^{+}$ \\
\hline \multicolumn{4}{|l|}{ Smoking $n=5,055$} \\
\hline Mean number of cigarettes & $\mathrm{T} 1 \mathrm{C}<\mathrm{NC}$ & $\mathrm{T} 2 \mathrm{C}<\mathrm{NC}$ & $\mathrm{FCC}<\mathrm{NC}$ \\
\hline Non-compliance & $\mathrm{T} 1 \mathrm{C}<\mathrm{NC}$ & $\mathrm{T} 2 \mathrm{C}<\mathrm{NC}$ & $\mathrm{FCC}<\mathrm{NC}$ \\
\hline
\end{tabular}

Note: $t: p<.10 ;{ }^{a}$ No statistically significant differences were found with regard to gender, educational level, income, and quality of life; ${ }^{b} \mathrm{~T} 1 \mathrm{C}=$ respondents who completed the $\mathrm{T1}$-measurement; $\mathrm{T} 2 \mathrm{C}=$ respondents who completed the $\mathrm{T2}$ measurement; $F C C=$ respondents who completed the T1- and the T2-measurement (fully complete cases); NC = respondents who did not complete the T1 or T2-measurement; a comma (",") means that the scores of the different conditions are equal; the ">" sign means that respondents in the first group (in front of the sign) were significantly older, more often in relationship, Dutch, having a higher BMI, suffering from a disease and having a healthier lifestyle ; the " $<$ " sign means that respondents in the first group (in front of the sign) were significantly less often employed, lived with fewer people in one household, reported a lower risk factor score and less non-compliance with the guidelines. 


\section{APPENDIX 3}

TO-T1 completers and T0-T2 completers

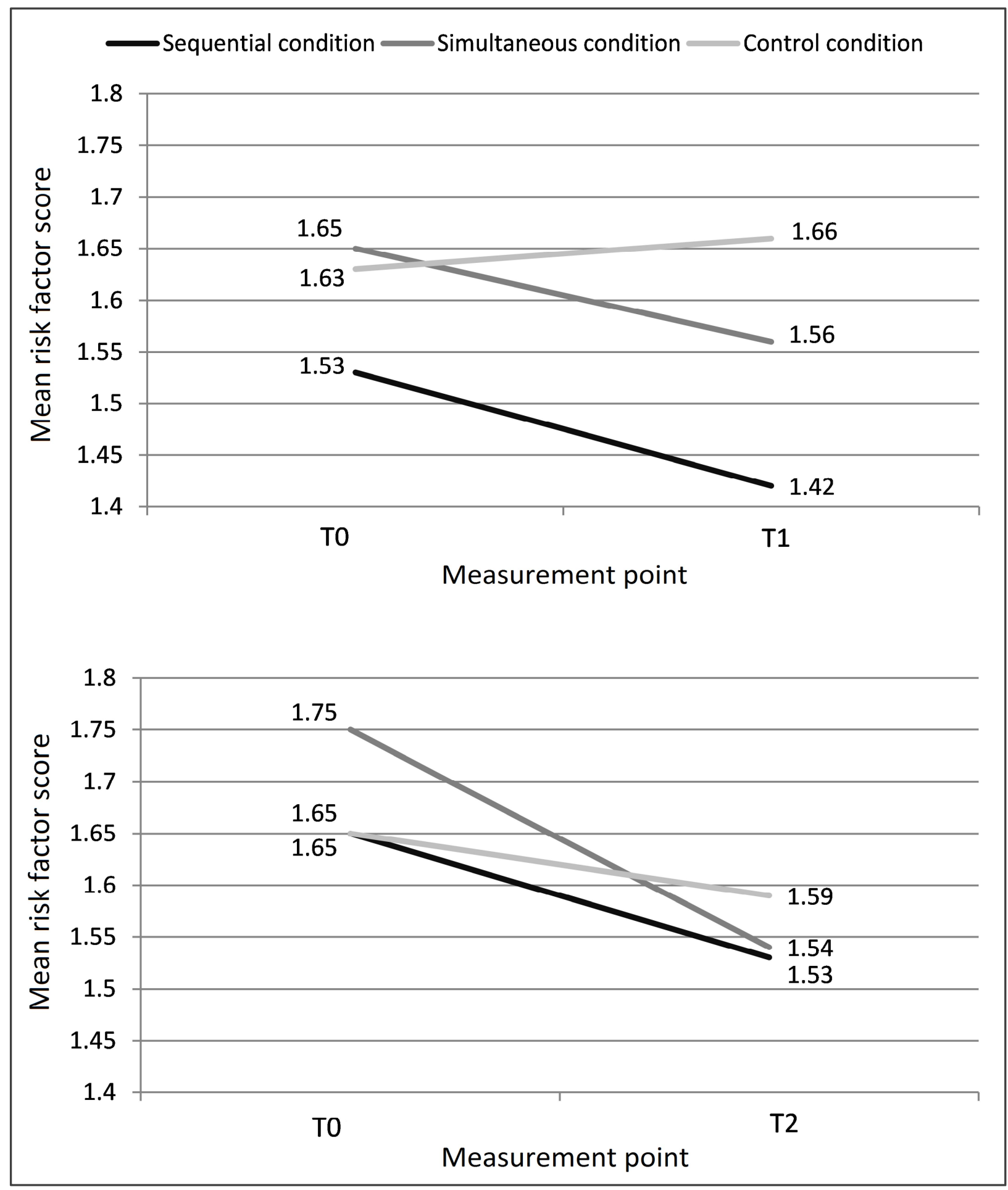

Figure I Mean number of risk factors among the different conditions (T1: $n=791 ; T 2: n=1,128)$ at baseline and follow-up at $\mathrm{T} 1$ and $\mathrm{T} 2$ respectively 
Table III Results of the linear regression analysis (top-down procedure method ${ }^{\text {a) }}$ with the risk factor index after 12 months $(n=768)$ and after 24 months $(n=1,113)$ as dependent variable

\begin{tabular}{|c|c|c|c|c|c|c|c|c|}
\hline \multirow[b]{2}{*}{ Variable } & \multicolumn{4}{|c|}{ After 12 months (T1) } & \multicolumn{4}{|c|}{ After 24 months (T2) } \\
\hline & $\beta$ & $p$ & $95 \% \mathrm{Cl}$ & ES & $\beta$ & $p$ & $95 \% \mathrm{Cl}$ & ES \\
\hline Condition & & $<.001$ & & & & $<.001$ & & \\
\hline SeqC vs. CC b & -.10 & .003 & $-0.35--0.07$ & 0.26 & -.04 & .19 & $-0.20-0.04$ & 0.10 \\
\hline SimC vs. CC b & -.07 & .03 & $-0.30--0.02$ & 0.20 & -.06 & .03 & $-0.25--0.01$ & 0.16 \\
\hline SeqC vs. SimC c & -.02 & .48 & $-0.20-0.09$ & 0.07 & .02 & .44 & $-0.08-0.17$ & 0.06 \\
\hline Age & -.06 & .047 & $-0.01--0.00$ & 0.01 & -.11 & $<.001$ & $-0.01--0.005$ & 0.01 \\
\hline Education (low vs. high) & -.08 & .02 & $-0.47--0.04$ & 0.32 & --- & --- & --- & --- \\
\hline Education (med vs. high) & -.03 & .40 & $-0.18-0.07$ & 0.07 & --- & --- & --- & --- \\
\hline Income (low vs. high) & .07 & .06 & $-0.01-0.34$ & 0.21 & .07 & .02 & $0.03-0.32$ & 0.21 \\
\hline Income (med vs. high) & .03 & .44 & $-0.09-0.20$ & 0.07 & .04 & .21 & $-0.04-0.19$ & 0.09 \\
\hline Work situation & --- & --- & --- & --- & -.05 & .04 & $-0.24--0.009$ & 0.15 \\
\hline K10 & -.06 & .07 & $-0.02-0.001$ & 0.01 & --- & --- & --- & --- \\
\hline Physical activity (min.) & --- & --- & --- & --- & -.09 & $<.001$ & $-0.001-0.000$ & 0.001 \\
\hline Vegetable intake (grams) & -.21 & $<.001$ & $-0.003--0.002$ & 0.002 & -.22 & $<.001$ & $-0.003--0.002$ & 0.004 \\
\hline Fruit intake (pieces) & -.30 & $<.001$ & $-0.29--0.19$ & 0.30 & -.29 & $<.001$ & $-0.28--0.20$ & 0.29 \\
\hline Alcohol intake (glasses) & .25 & $<.001$ & $0.11-0.18$ & 0.18 & .27 & $<.001$ & $0.16-0.23$ & 0.24 \\
\hline Smoking (cigarettes) & .25 & $<.001$ & $0.04-0.07$ & 0.07 & .20 & $<.001$ & $0.03-0.05$ & 0.05 \\
\hline$R^{2}$ & & & & .37 & & & & .36 \\
\hline
\end{tabular}

Note: ${ }^{a}$ All variables regarding demographics, health status and lifestyle behaviour were included in the most extensive model; ${ }^{b}$ The control condition was the reference category; ${ }^{c}$ The simultaneous condition was the reference category.

Table IV Results of the logistic regression analysis (top-down procedure ${ }^{\text {a) }}$ with compliance with the five different guidelines (yes $=1 ; n o=0)$ after 12 months $(n \geq 760)$ and after 24 months ( $n \geq 1,106)$ as dependent variables

\begin{tabular}{|c|c|c|c|c|c|c|c|c|c|}
\hline \multirow{2}{*}{$\begin{array}{l}\text { Lifestyle } \\
\text { behaviour }\end{array}$} & \multirow[t]{2}{*}{ Group } & \multicolumn{4}{|c|}{ T0-T1-completers } & \multicolumn{4}{|c|}{ T0-T2-completers } \\
\hline & & $\begin{array}{l}\text { OR for } \\
\text { change }\end{array}$ & $p$ & $95 \% \mathrm{Cl}$ & ES & $\begin{array}{l}\text { OR for } \\
\text { change }\end{array}$ & $p$ & $95 \% \mathrm{Cl}$ & ES \\
\hline \multirow{4}{*}{$\begin{array}{l}\text { Physical } \\
\text { activity }\end{array}$} & Condition & & .36 & & & & .71 & & \\
\hline & SeqC vs. CC b & 1.53 & .19 & $0.81-2.89$ & 0.23 & 0.96 & .86 & $0.57-1.61$ & 0.02 \\
\hline & SimC vs. CC ${ }^{b}$ & 1.36 & .30 & $0.76-2.45$ & 0.17 & 1.18 & .52 & $0.71-1.95$ & 0.09 \\
\hline & SeqC vs.Sim C ${ }^{c}$ & 1.12 & .74 & $0.57-2.21$ & 0.06 & 0.81 & .43 & $0.48-1.37$ & 0.12 \\
\hline \multirow[t]{4}{*}{ Vegetable } & Condition & & .09 & & & & .14 & & \\
\hline & SeqC vs. CC ${ }^{b}$ & 1.58 & .03 & $1.05-2.39$ & 0.25 & 1.15 & .42 & $0.82-1.63$ & 0.08 \\
\hline & SimC vs. CC ${ }^{b}$ & 1.34 & .18 & $0.88-2.03$ & 0.16 & 1.40 & .048 & $1.00-1.94$ & 0.18 \\
\hline & SeqC vs.SimC c & 1.18 & .44 & $0.78-1.81$ & 0.09 & 0.83 & .28 & $0.58-1.17$ & 0.11 \\
\hline \multirow[t]{4}{*}{ Fruit } & Condition & & .15 & & & & .04 & & \\
\hline & SeqC vs. CC b & 1.48 & .06 & $0.99-2.23$ & 0.22 & .94 & .71 & $0.67-1.32$ & 0.04 \\
\hline & SimC vs. CC b & 1.28 & .22 & $0.86-1.91$ & 0.14 & 1.43 & .04 & $1.02-2.01$ & 0.20 \\
\hline & SeqC vs.Sim $C^{c}$ & 1.16 & .49 & $0.76-1.76$ & 0.08 & 0.66 & .02 & $0.46-0.94$ & 0.23 \\
\hline \multirow[t]{4}{*}{ Alcohol } & Condition & & .17 & & & & .08 & & \\
\hline & SeqC vs. CC b & 1.38 & .28 & $0.77-2.47$ & 0.18 & 1.75 & .03 & $1.07-2.86$ & 0.31 \\
\hline & SimC vs. $\mathrm{CC}$ b & 1.72 & .07 & $0.97-3.05$ & 0.30 & 1.31 & .25 & $0.83-2.08$ & 0.15 \\
\hline & SeqC vs.SimC c & 0.80 & .50 & $0.43-1.51$ & 0.12 & 1.34 & .27 & $0.80-2.24$ & 0.16 \\
\hline \multirow[t]{4}{*}{ Smoking } & Condition & & .34 & & & & .02 & & \\
\hline & SeqC vs. CC ${ }^{b}$ & 0.83 & .67 & $0.34-2.00$ & 0.10 & 1.52 & .22 & $0.78-2.94$ & 0.23 \\
\hline & SimC vs. CC ${ }^{b}$ & 0.55 & .15 & $0.24-1.25$ & 0.33 & 0.60 & .07 & $0.35-1.04$ & 0.28 \\
\hline & SeqC vs.Sim C c & 1.50 & .33 & $0.66-3.44$ & 0.22 & 2.52 & .005 & $1.32-4.82$ & 0.51 \\
\hline
\end{tabular}

Note: ${ }^{a}$ All variables regarding demographics, health status and lifestyle behaviour were included in the most extensive model;

$b$ The control condition was the reference category; ${ }^{c}$ The simultaneous condition was the reference category. 


\section{APPENDIX 4}

Fully complete cases

$\longrightarrow$ Sequential condition $\longrightarrow$ Simultaneous condition - Control condition

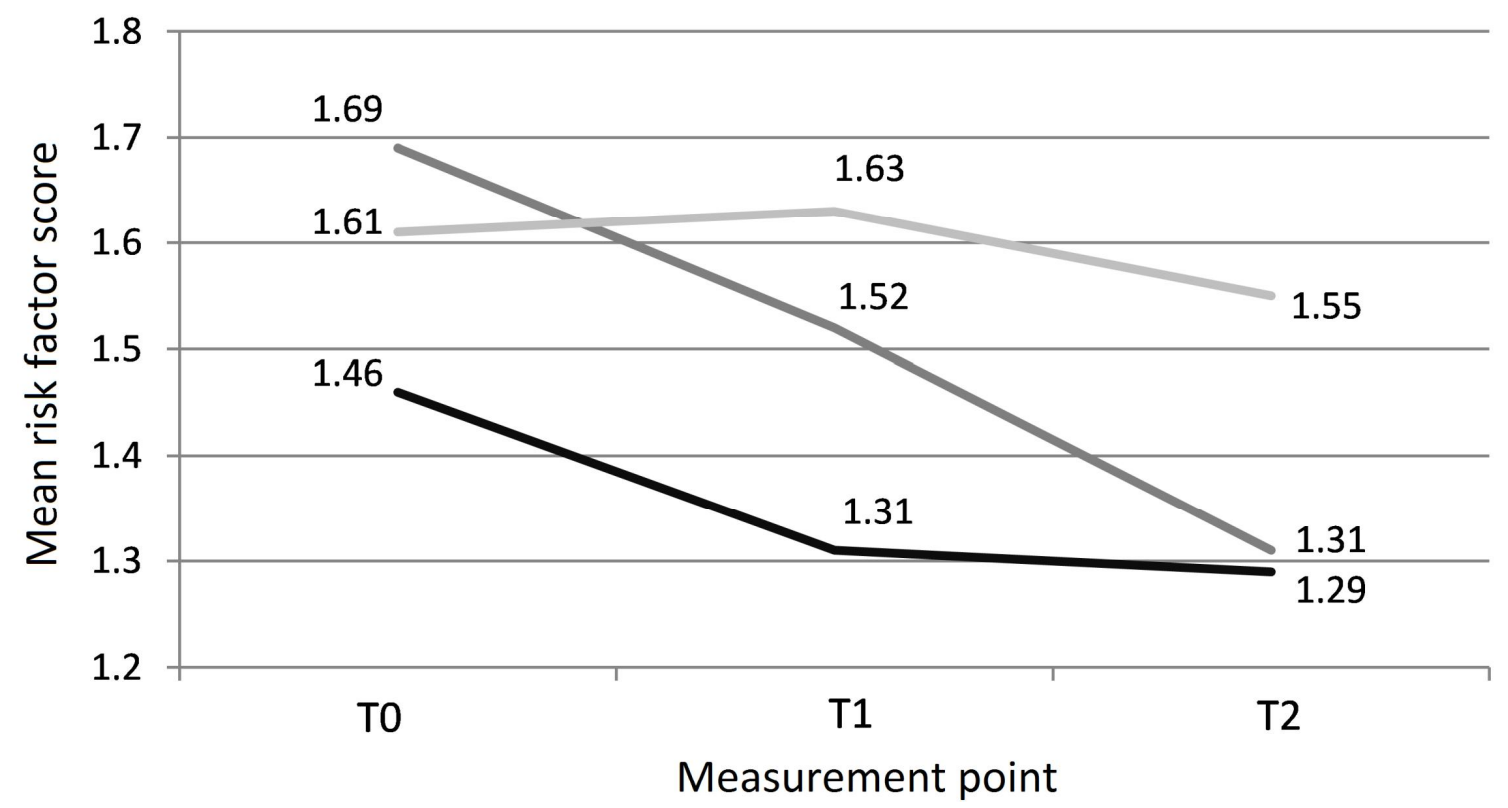

Figure II Mean number of risk factors among the different conditions (complete cases: $n=475$ ) at baseline and follow-up (sequential condition $n=140$; simultaneous condition $n=143$; control condition $n=192$ ) 
Table $\mathrm{V}$ Results of the linear regression analysis (top-down procedure ${ }^{\mathrm{a}}$ ) with the risk factor index after 12 months and after 24 months as dependent variable $(n=466)$

\begin{tabular}{|c|c|c|c|c|c|c|c|c|}
\hline \multirow[b]{2}{*}{ Variable } & \multicolumn{4}{|c|}{ After 12 months (T1) } & \multicolumn{4}{|c|}{ After 24 months (T2) } \\
\hline & $\beta$ & $\mathrm{P}$ & $95 \% \mathrm{Cl}$ & ES & $\beta$ & $p$ & $95 \% \mathrm{Cl}$ & ES \\
\hline Condition & & $<.001$ & & & & $<.001$ & & \\
\hline SeqC vs. CC b & -.11 & .006 & $-0.42--0.07$ & 0.31 & -.09 & .03 & $-0.38--0.03$ & 0.26 \\
\hline $\operatorname{SimC}$ vs. $\mathrm{CC}^{\mathrm{b}}$ & -.09 & .03 & $-0.36--0.02$ & 0.24 & -.14 & .001 & $-0.48--0.13$ & 0.39 \\
\hline SeqC vs. SimC c & -.02 & .59 & $-0.24-0.13$ & 0.07 & .05 & .27 & $-0.08-0.29$ & 0.13 \\
\hline Age & -.07 & .049 & $-0.01-0.000$ & 0.01 & --- & --- & --- & --- \\
\hline Education (low vs. high) & -.08 & .04 & $-0.58--0.02$ & 0.38 & --- & --- & --- & --- \\
\hline Education (med vs. high) & -.04 & .36 & $-0.23-0.08$ & 0.09 & --- & --- & --- & --- \\
\hline Income (low vs. high) & .09 & .048 & $0.002-0.42$ & 0.27 & .09 & .03 & $0.02-0.43$ & 0.28 \\
\hline Income (med vs. high) & .07 & .11 & $-0.03-0.32$ & 0.18 & .11 & .02 & $0.04-0.38$ & 0.27 \\
\hline Work situation & --- & --- & --- & --- & -.07 & .08 & $-0.30-0.02$ & 0.18 \\
\hline Physical activity (min.) & -.06 & .09 & $-0.001-0.000$ & 0.00 & -.09 & .02 & $-0.001-0.000$ & 0.001 \\
\hline Vegetable intake (grams) & -.25 & $<.001$ & $-0.004--0.002$ & 0.003 & -.27 & $<.001$ & $-0.004--0.002$ & 0.004 \\
\hline Fruit intake (pieces) & -.30 & $<.001$ & $-0.31--0.19$ & 0.31 & -.31 & $<.001$ & $-0.31--0.19$ & 0.32 \\
\hline Alcohol intake (glasses) & .31 & $<.001$ & $0.17-0.28$ & 0.28 & .26 & $<.001$ & $0.14-0.24$ & 0.24 \\
\hline Smoking (cigarettes) & .27 & $<.001$ & $0.05-0.08$ & 0.08 & .22 & $<.001$ & $0.03-0.07$ & 0.06 \\
\hline$R^{2}$ & & & & .43 & & & & .40 \\
\hline
\end{tabular}

Note: ${ }^{a}$ All variables regarding demographics, health status and lifestyle behaviour were included in the most extensive model;

${ }^{b}$ The control condition was the reference category; ${ }^{c}$ The simultaneous condition was the reference category.

Table VI Results of the logistic regression analysis (top-down procedure a) with compliance with the five different guidelines (yes $=1 ;$ no $=0$ ) after 12 months and after 24 months as dependent variables $(n=457)$

\begin{tabular}{|c|c|c|c|c|c|c|c|c|c|}
\hline \multirow{2}{*}{$\begin{array}{l}\text { Lifestyle } \\
\text { behaviour }\end{array}$} & \multirow[t]{2}{*}{ Group } & \multicolumn{4}{|c|}{ After 12 months (T1) } & \multicolumn{4}{|c|}{ After 24 months (T2) } \\
\hline & & $\begin{array}{l}\text { OR for } \\
\text { change }\end{array}$ & $p$ & $95 \% \mathrm{Cl}$ & ES & $\begin{array}{l}\text { OR for } \\
\text { change }\end{array}$ & $p$ & $95 \% \mathrm{Cl}$ & ES \\
\hline \multirow[t]{4}{*}{ Phys. act. } & Condition & & .82 & & & & .31 & & \\
\hline & SeqC vs. CC b & 1.32 & .53 & $0.55-3.15$ & 0.15 & 0.76 & .58 & $0.28-2.03$ & 0.15 \\
\hline & SimC vs. CC b & 1.05 & .90 & $0.48-2.30$ & 0.03 & 1.76 & .26 & $0.66-4.68$ & 0.31 \\
\hline & SeqC vs.SimC ${ }^{c}$ & 1.26 & .50 & $0.46-3.15$ & 0.13 & 0.43 & .14 & $0.14-1.31$ & 0.46 \\
\hline \multirow[t]{4}{*}{ Vegetable } & Condition & & .06 & & & & .06 & & \\
\hline & SeqC vs. CC b & 1.69 & .05 & $1.00-2.86$ & 0.29 & 1.06 & .84 & $0.62-1.81$ & 0.03 \\
\hline & $\operatorname{SimC}$ vs. $\mathrm{CC}^{\mathrm{b}}$ & 1.75 & .04 & $1.03-2.98$ & 0.31 & 1.83 & .03 & $1.08-3.11$ & 0.33 \\
\hline & SeqC vs.Sim C ${ }^{c}$ & 0.96 & .89 & $0.56-1.66$ & 0.02 & 0.58 & .06 & $0.33-1.02$ & 0.30 \\
\hline \multirow[t]{4}{*}{ Fruit } & Condition & & .55 & & & & .07 & & \\
\hline & SeqC vs. CC b & 1.23 & .44 & $0.73-2.09$ & 0.12 & 1.67 & .06 & $0.97-2.85$ & 0.28 \\
\hline & $\operatorname{SimC}$ vs. CC b & 1.32 & .30 & $0.78-2.21$ & 0.15 & 1.70 & .048 & $1.00-2.88$ & 0.29 \\
\hline & SeqC vs.SimC ${ }^{c}$ & 0.94 & .82 & $0.54-1.64$ & 0.04 & 0.98 & .94 & $0.55-1.74$ & 0.01 \\
\hline \multirow[t]{4}{*}{ Alcohol } & Condition & & .07 & & & & .003 & & \\
\hline & SeqC vs. CC ${ }^{b}$ & 2.77 & .02 & $1.16-6.65$ & 0.56 & 3.85 & .002 & $1.62-9.16$ & 0.74 \\
\hline & $\operatorname{SimC}$ vs. $C^{b}$ & 1.66 & .19 & $0.77-3.58$ & 0.28 & 2.68 & .01 & $1.23-5.84$ & 0.54 \\
\hline & SeqC vs.Sim C ${ }^{c}$ & 1.67 & .28 & $0.65-4.26$ & 0.28 & 1.44 & .45 & $0.57-3.65$ & 0.20 \\
\hline \multirow[t]{4}{*}{ Smoking } & Condition & & .27 & & & & .27 & & \\
\hline & SeqC vs. CC b & 0.67 & .53 & $0.19-2.36$ & 0.22 & 1.37 & .62 & $0.40-4.65$ & 0.17 \\
\hline & $\operatorname{SimC}$ vs. $C^{b}$ & 0.41 & .11 & $0.13-1.24$ & 0.50 & 0.56 & .25 & $0.21-1.51$ & 0.32 \\
\hline & SeqC vs.Sim C ${ }^{c}$ & 1.64 & .39 & $0.53-5.08$ & 0.27 & 2.44 & .13 & $0.76-7.83$ & 0.49 \\
\hline
\end{tabular}

Note: ${ }^{a}$ All variables regarding demographics, health status and lifestyle behaviour were included in the most extensive model;

${ }^{b}$ The control condition was the reference category; ${ }^{c}$ The simultaneous condition was the reference category. 


\section{APPENDIX 5}

Table VII Cost prices for the different types of health care and absenteeism from work

\begin{tabular}{lll}
\hline Health care and absenteeism from work & $\begin{array}{l}\text { Price per visit } \\
\text { (in €) } \text { a }^{\text {a }}\end{array}$ & $\begin{array}{l}\text { Travel distance } \\
\text { (in km) }\end{array}$ \\
\hline General practitioner or practice nurse (by phone, in the practice, at home) & 30.92 & 1.1 \\
Medical specialist (e.g. cardiologist, rheumatologist, internist, neurologist) & 34.38 & 7.0 \\
Alternative care giver (e.g. homeopath, acupuncturist) & $55.33^{\mathrm{c}}$ & 2.2 \\
Paramedical care giver (e.g. physiotherapist, ergo therapist, speech therapist) & 33.40 & 2.2 \\
Dietitian & 29.46 & 2.2 \\
Mental health care giver (psychologist, psychiatrist, psychotherapist) & 83.37 & 5.0 \\
Clinic for alcohol and drugs (CAD) & $76.39^{\mathrm{c}}$ & 5.0 \\
Other care givers & various ${ }^{\mathrm{d}}$ & various $^{\mathrm{d}}$ \\
Hospital stay (per day) & 551.10 & 7.0 \\
Revalidation centre (per day) & 371.04 & 7.0 \\
Other paid care (e.g. professional home care; per hour) & 38.20 & $\mathrm{n} / \mathrm{a}$ \\
Other unpaid care (from family, friends; per hour) & 13.64 & $\mathrm{n} / \mathrm{a}$ \\
Sickness absenteeism from work (in days) & $262.08^{\mathrm{e}}$ & $\mathrm{n} / \mathrm{a}$ \\
Medication ${ }^{\mathrm{d}}$ & various ${ }^{\mathrm{f}}$ & $\mathrm{n} / \mathrm{a}$ \\
\hline
\end{tabular}

Note: a Dutch Manual for Cost Analysis in Health Care (Hakkaart-Van Roijen et al., 2010); ${ }^{b}$ Costs per km: $0.20 €$ (HakkaartVan Roijen et al., 2010; National Institute for Public Health and the Environment, 2008); c Mean price was calculated based on three prices, price details are available upon request; ${ }^{d}$ Price depended on the type of care giver, price details are available upon request; " Calculated via the human capital method using mean costs for the Dutch population corrected for gender and age; ${ }^{f}$ Costs of medications were calculated based on the dose described by the respondent (per tablet, gram or ml), price was look up at www.medicijnkosten.nl, price details are available upon request. 



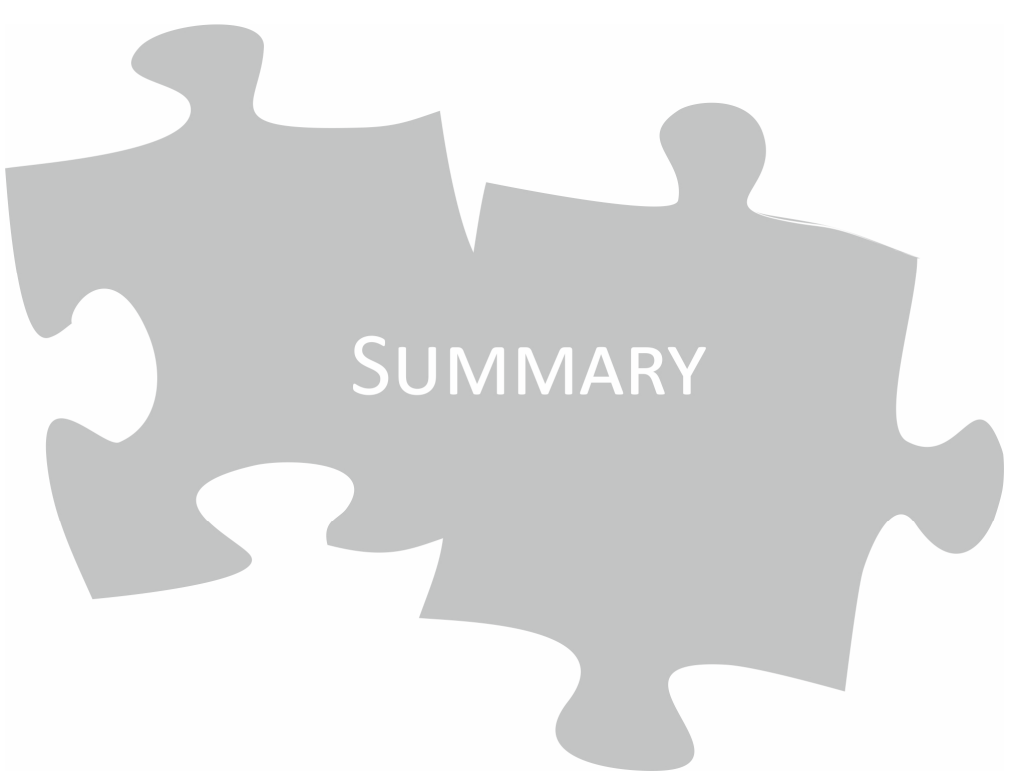



Non-communicable chronic diseases, such as cardiovascular diseases, cancer and diabetes, are often caused by health risk behaviours, such as physical inactivity, unhealthy diet, excessive alcohol intake and tobacco use. To enhance lifestyle behaviours among adults, a web-based computer-tailored intervention was developed and tested. This intervention aimed at the lifestyle behaviours mentioned above. In this dissertation, the process of development of this intervention is described (chapter 2), the adoption and dissemination of the intervention via different Regional Health Authorities (Dutch: GGD) is studied (chapter 3), the use of the intervention is explored (chapters 4 and 5) and the intervention is evaluated by studying its effectiveness (chapters 6 and 7) as well as its costeffectiveness (chapter 8).

Chapter 1 provides a general introduction to the topic of this dissertation. Background information is provided about multiple behaviour change and computer-tailoring. The theoretical framework of the studies - the I-Change model - is introduced. The aim of the dissertation and the research questions are presented.

Chapter 2 includes the study protocol of the intervention. The development of the multiple behaviour change intervention, called myHealthyBehaviour (Dutch: "mijnGezondGedrag"), is described. This web-based computer-tailored lifestyle intervention was aimed at increasing physical activity levels, fruit consumption and vegetable consumption, decreasing alcohol intake and quitting smoking.

Chapter 3 describes the dissemination strategy used in our study. Dissemination of the intervention programme took place via the Adult Health Monitor of different Regional Health Authorities. This strategy has been shown to be an appropriate method to disseminate the intervention and to reach adults. The levels of reach were high: around one third $(n>5,000)$ of the respondents who completed the online Adult Health Monitor logged in to our intervention. However, completion rates at baseline, as well as re-visits to the intervention, were low. Males, older respondents, more highly educated participants and those with a healthier lifestyle and a healthier Body Mass Index were most likely to participate in the intervention. This means that reach of at-risk individuals (e.g. those of low socioeconomic status and with an unhealthy lifestyle) was modest.

Chapter 4 describes a study comparing completion rates among the sequential and simultaneous interventions. Both interventions suffered from high baseline incompletion rates. In the sequential condition, $65.0 \%$ did not complete the intervention at baseline; in the simultaneous condition, $77.1 \%$ did not complete the first session. In both conditions, discontinuation was predicted by lower age and an unhealthier lifestyle. In the sequential intervention, non-completion was also predicted by being male. When respondents failed to adhere to at least two of the guidelines, those receiving the simultaneous intervention were more inclined to drop out than were those receiving the sequential intervention. Possible reasons for the higher dropout rate in our simultaneous intervention may be the amount of time required and information overload.

Chapter 5 presents a study in which individuals' preferences for particular lifestyle modules within the multiple behaviour change intervention were investigated. The sequential condition was included in this study. Respondents had the choice to select one of the five modules during their baseline visit. 
The physical activity module was the most popular, followed by the smoking and fruit modules. Young adults tended to prefer the physical activity and fruit modules, whereas the vegetable module was more popular among older adults. No consistent pattern was identified for the alcohol and smoking modules. The results of this study give a preliminary indication that physical activity may serve as a gateway behaviour when aiming at multiple behaviour change in eHealth interventions.

Chapter 6 presents a randomized controlled trial $(R C T ; n=448)$ investigating the effectiveness of a web-based tailored alcohol intervention after six months. This intervention aimed at alcohol reduction in adults reporting too high alcohol intake at baseline. Two tailoring strategies were compared: an alternating condition (i.e. the tailored feedback was split up into a series of messages discussing individual topics offered while the respondent was filling out the programme) and a summative condition (i.e. all advice was presented at once after the respondent had answered all questions within the intervention). Complete case analyses revealed that web-based tailored feedback can be an effective way to reduce alcohol intake among adults. However, this effect was not confirmed when applying the multiple imputation technique to deal with missing values. There was no indication that either one of the tailoring strategies was more effective in lowering alcohol intake, and programme appreciation was comparable for the two experimental groups. Nevertheless, the lower attrition rates we found during the first visit suggest that the version of the intervention with alternating questions and advice may be preferred.

Chapter 7 presents an RCT testing the effects of our web-based computer-tailored lifestyle intervention for adults after 12 and 24 months. Three groups were included: the sequential condition; the simultaneous condition; and the control condition. Both tailoring strategies were found to be effective in improving lifestyle. The sequential condition resulted in the most significant effects in comparison to the control condition after 12 months ( $E S=0.30$ ). After 24 months the simultaneous condition was most effective ( $E S=0.19$ ). The effectiveness of both types of intervention implies that providers can use the strategy that suits their particular circumstances best. However, which intervention is best may depend on the targeted behaviour or personal preferences.

Chapter 8 describes the results of an economic evaluation of our web-based computer-tailored lifestyle intervention. Cost-effectiveness and cost-utility were investigated. The results of this study give an indication that the two tailored intervention programmes were likely to be more cost-effective than a control group in which respondents received a short tailored overview regarding their current lifestyle. In general, the simultaneous intervention was likely to be most cost-effective, followed by the sequential intervention. With incremental costs of $€ 4,594$ per gain in lifestyle score by meeting additional public health guidelines, the sequential condition might be most likely to be cost-effective; with incremental costs of $€ 17,106$ or higher, the simultaneous version of the web-based intervention might be more likely to be cost-effective than the sequential version. Regarding cost utilities, the intervention received by the control group might be most preferable when compared to both lifestyle interventions (sequential and simultaneous).

Chapter 9 provides a general discussion of this dissertation. The results of the different studies (chapters 2 to 8), including their limitations, are discussed. Recommendations for theory, future 
research and practice are given. The following conclusions were drawn: it is essential to further optimize reach of web-based computer-tailored interventions aimed at multiple lifestyle behaviours by putting additional effort into increasing interest in the lifestyle intervention among at-risk individuals and encouraging them to actually use the intervention; therefore, strategies to optimize programme completion as well as continued use of computer-tailored interventions should be studied. In multiple behaviour change interventions, physical activity may serve as a gateway behaviour in tailored eHealth lifestyle promotion. The web-based computer-tailored lifestyle intervention has proven to be effective as well as cost-effective. No distinct tailoring strategy receives preference and therefore, both types of intervention might be chosen for implementation.

The main conclusions of this dissertation can be summarized as the following: our interventions showed positive results regarding their reach, appreciation, effectiveness and cost-effectiveness. A combination of both the sequential and the simultaneous tailoring strategies may be most suitable for multiple behaviour change. However, the interventions suffered from high dropout rates, resulting in high numbers of missing values, and selective dropout occurred. Therefore, the results have to be interpreted cautiously. Much work remains to understand the low levels of engagement in web-based interventions, and statistical methods for dealing with missing values need further attention. 



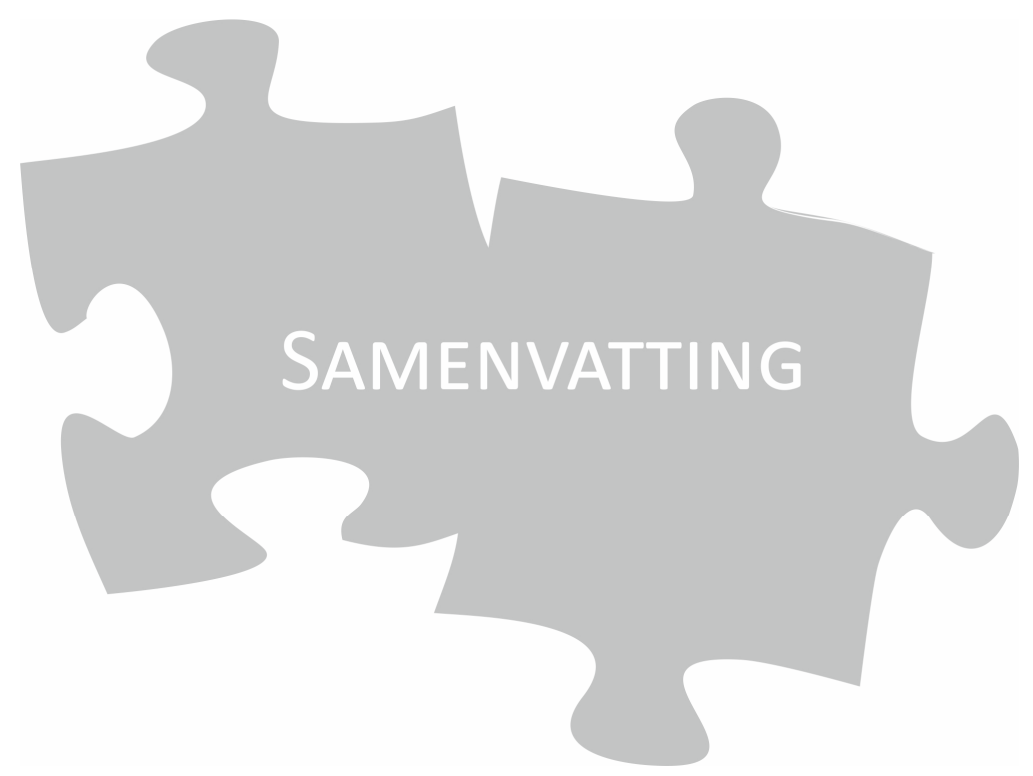



Chronische ziektes zoals cardiovasculaire aandoeningen, kanker en diabetes, worden vaak veroorzaakt door gezondheidsrisicogedragingen, zoals lichamelijke inactiviteit, ongezonde voeding, overmatige alcoholconsumptie en tabaksgebruik. Om gezonder leefstijlgedrag bij volwassenen te stimuleren hebben we een webgebaseerde advies-op-maat-interventie ontwikkeld en getest. Deze interventie richtte zich op bovengenoemde leefstijlgedragingen. In dit proefschrift wordt het ontwikkelproces en de evaluatie van de interventie beschreven (hoofdstuk 2) en worden de adoptie en disseminatie van de interventie via verschillende GGDen bestudeerd (hoofdstuk 3). Nadat het gebruik van de interventie in kaart wordt gebracht (hoofdstukken 4 en 5), wordt deze geëvalueerd op effectiviteit (hoofdstukken 6 en 7) en op kosteneffectiviteit (hoofdstuk 8).

Hoofdstuk 1 geeft een algemene introductie met betrekking tot het onderwerp van dit proefschrift. Achtergrondinformatie over multipele gedragsverandering en 'computer-tailoring' wordt aan de lezer gepresenteerd. Het theoretische kader van de studies - het I-Change model - wordt geïntroduceerd. Tot slot worden het doel van het proefschrift en de onderzoeksvragen gepresenteerd.

Hoofdstuk 2 omvat het studieprotocol van de interventie. De ontwikkeling van de multipele gedragsveranderingsinterventie, genaamd "mijnGezondGedrag", wordt beschreven. De webgebaseerde advies-op-maat-leefstijlinterventie had als doel het verhogen van lichamelijke activiteit en fruit- en groenteconsumptie, verminderen van alcoholgebruik en stoppen met roken.

Hoofdstuk 3 beschrijft de disseminatiestrategie die gebruikt werd in onze studie. Disseminatie van de interventie vond plaats via de Gezondheidsenquête van verschillende GGDen. De resultaten laten zien dat deze strategie een geschikte methode is voor het verspreiden van de interventie en het bereiken van volwassenen. Het bereik was hoog: ongeveer een derde $(n>5.000)$ van de respondenten die hebben geparticipeerd aan de online Gezondheidsenquête, hebben tevens ingelogd in het programma. De mate van voltooiing tijdens de nulmeting en herbezoeken aan het programma (de interventie) waren daarentegen laag. Mannen, oudere respondenten, hoger opgeleide respondenten en respondenten met een gezondere leefstijl en een gezondere Body Mass Index waren eerder geneigd om deel te nemen aan de interventie. Dit betekent dat het bereik van risicogroepen (bijvoorbeeld mensen met een lage sociaaleconomische status en/of een ongezondere leefstijl) matig was.

Hoofdstuk 4 beschrijft een studie waarin de uitval en voltooiing van de sequentiële interventie met die van de simultane interventie worden vergeleken. Beide interventies vertoonden een hoge mate van uitval tijdens de nulmeting. In de sequentiële conditie had 65,0\% de interventie niet compleet ingevuld; in de simultane conditie had $77,1 \%$ de eerste sessie niet compleet ingevuld. In beide condities werd uitval voorspeld door een jongere leeftijd en een ongezondere leefstijl. In de sequentiële conditie werd dit verder voorspeld door geslacht; mannen bleken eerder geneigd om het programma vroegtijdig af te breken. Wanneer respondenten aan twee of meer richtlijnen niet voldeden, waren respondenten in de simultane interventie eerder geneigd om uit te vallen dan respondenten die de sequentiële interventie hebben gekregen. Mogelijke redenen voor het hogere aantal uitvallers in de simultane interventie zouden benodigde tijd en informatieoverbelasting kunnen zijn. 
Hoofdstuk 5 presenteert een studie waarin individuele voorkeuren voor bepaalde leefstijlmodules binnen de multipele veranderingsinterventie werden onderzocht. Alleen de sequentiële conditie werd geïncludeerd in deze studie. Tijdens het eerste bezoek (nulmeting) hadden respondenten de mogelijkheid om één van de vijf modules te kiezen. De beweegmodule was het meest populair, gevolgd door de rookmodule en de fruitmodule. Jongvolwassenen leken eerder de beweegmodule en de fruitmodule te verkiezen, terwijl de groentemodule populairder was onder oudere volwassenen. Voor de alcoholmodule en de rookmodule werd geen consistent patroon in voorkeur gevonden. De resultaten van deze studie geven een voorlopige indicatie dat beweging gebruikt kan worden als eerste gedrag om ook ander gezondheidsgedrag te kunnen veranderen (het zgn. gateway effect) in eHealth-interventies.

Hoofdstuk 6 presenteert een gerandomiseerd onderzoek met controlegroep (RCT; $n=448$ ) waarin de effectiviteit van een webgebaseerde advies-op-maat-alcoholinterventie na zes maanden werd getest. Deze interventie richtte zich op de vermindering van alcoholconsumptie bij volwassenen die op baseline te hoge alcoholinname vertoonden. Twee tailoringstrategieën werden met elkaar vergeleken: een afwisselende conditie (d.w.z. dat het advies-op-maat werd opgesplitst in een serie van adviezen die verschillende onderwerpen behandelden, terwijl de respondent het programma invulde) en een summatieve conditie (d.w.z. dat alle adviezen in één keer getoond werden, nadat de respondent alle vragen binnen de interventie had beantwoord). De complete case-analyses lieten zien dat webgebaseerd advies-op-maat een effectieve methode is voor het verminderen van alcoholgebruik bij volwassenen. Dit effect werd echter niet gevonden wanneer de missende waardes werden vervangen middels een multipele imputatietechniek. Verder bleek dat géén van de tailoringstrategieën verschilde qua effectiviteit in het reduceren van alcoholinname. Ook was de waardering van het programma vergelijkbaar tussen de twee experimentele groepen. Desalniettemin werd een lagere attritie gevonden tijdens de nulmeting, hetgeen een indicatie kan zijn dat de interventie waarin vragen en adviezen afwisselend werden getoond, geprefereerd werd.

Hoofdstuk 7 beschrijft een gerandomiseerd onderzoek met controlegroep waarin de effectiviteit van onze webgebaseerde advies-op-maat-leefstijlinterventie werd getest onder volwassenen na 12 en 24 maanden. Het onderzoek bestond uit drie groepen: de sequentiële conditie, de simultane conditie en de controleconditie. De resultaten toonden dat beide tailoringstrategieën effectief waren in het verbeteren van leefstijl. Na 12 maanden resulteerde de sequentiële conditie in de meest significante effecten in vergelijking met de controleconditie $(E S=0,30)$. Na 24 maanden bleek de simultane conditie het meest effectief te zijn $(E S=0,19)$. De effectiviteit van beide types interventies impliceert dat gezondheidsbevorderaars beide methodes kunnen gebruiken. Er wordt geopperd dat de effectiviteit van de interventiemethodiek afhankelijk zou kunnen zijn van het doelgedrag of persoonlijke voorkeuren van de respondent.

Hoofdstuk 8 beschrijft de resultaten van de economische evaluatie van onze webgebaseerde computer-getailorde leefstijlinterventie. De kosteneffectiviteit en de kostenutiliteit van beide tailoringmethoden worden beschreven. De resultaten laten zien dat beide advies-op-maatprogramma's kosteneffectiever waren dan de controlegroep, waarin respondenten een kort 
getailored overzicht met betrekking tot hun huidige leefstijl hebben ontvangen. In het algemeen was de simultane interventie de meest kosteneffectieve methode, gevolgd door de sequentiële interventie. Met incrementele kosten van €4.594 per verbetering in leefstijlscore (door het voldoen aan een extra richtlijn) was de sequentiële conditie waarschijnlijk het meest kosteneffectief; met incrementele kosten van $€ 17.106$ of meer was de simultane versie van de webgebaseerde interventie waarschijnlijk kosteneffectiever dan de sequentiële versie. Wat betreft kostenutiliteit kreeg de interventie die de controlegroep had ontvangen de voorkeur in vergelijking met beide leefstijlinterventies (zowel sequentieel als simultaan).

Hoofdstuk 9 bevat een algemene discussie van dit proefschrift. De resultaten van de verschillende studies (hoofdstukken 2 tot en met 8) worden bediscussieerd. Aanbevelingen voor theorie, toekomstig onderzoek en praktijk worden gegeven. De resultaten van de studies impliceren dat het essentieel is om het bereik van webgebaseerde advies-op-maat-interventies die zich richten op meerdere leefstijlgedragingen verder te optimaliseren door het verhogen van interesse in leefstijlinterventies bij volwassenen en hen te stimuleren de interventie daadwerkelijk te gebruiken. Om dit te bereiken zouden vooraleerst effectieve strategieën ter adoptie, voltooiing en herhaald gebruik van dit soort programma's geïdentificeerd moeten worden. In multipele gedragsveranderingsinterventies zou lichamelijke activiteit als gateway behaviour kunnen dienen in getailorde bevordering van gezonde leefstijl via eHealth. Tot slot, beide webgebaseerde computer-getailorde leefstijlinterventies waren effectief en kosteneffectief, en kunnen derhalve beiden gekozen worden voor implementatie.

De hoofdconclusies van dit proefschrift kunnen als volgt worden samengevat: Onze interventies hebben positieve resultaten laten zien met betrekking tot bereik, waardering, effectiviteit en kosteneffectiviteit. Een combinatie van de sequentiële en de simultane tailoringstrategie zou het meest geschikt kunnen zijn voor multipele gedragsverandering. Daar tegenover staat dat de interventies lijden aan hoge uitval, resulterend in een hoog aantal missende waardes. Bovendien was er sprake van selectieve uitval. Om deze redenen dienen de resultaten met enige voorzichtigheid te worden geïnterpreteerd. $\mathrm{Er}$ is onderzoek nodig om de lage niveaus van betrokkenheid in webgebaseerde interventies te begrijpen en te verminderen. Daarenboven is er aandacht nodig voor onderzoek naar de beste statistische methodes om met missende waardes om te gaan. 



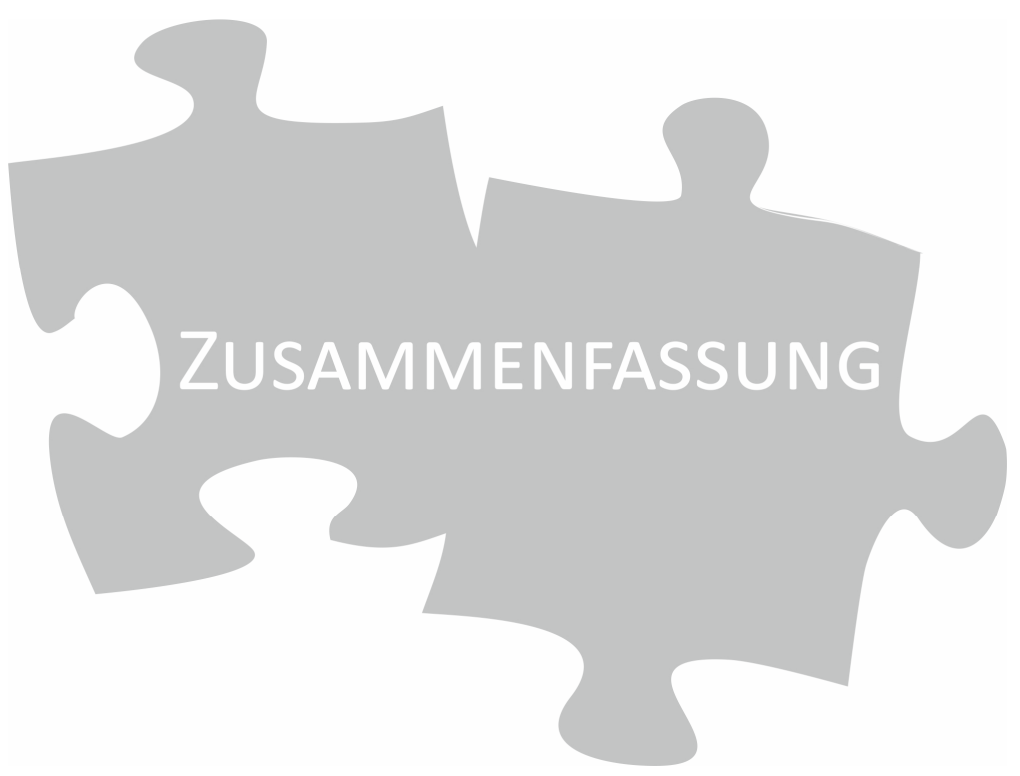



Chronische Krankheiten, wie zum Beispiel Herz- und Gefäßkrankheiten, Krebs und Diabetes, werden oft durch ungesunde Verhaltensweisen, wie körperliche Inaktivität, ungesunde Ernährung und zu hohen Alkohol- und Tabakkonsum, verursacht. Um Erwachsene bei einem gesunden Lebensstil zu unterstützen, wurde im Rahmen dieser Dissertation eine web-basierte Intervention mit maßgeschneiderten Feedbacktexten entwickelt und evaluiert. Diese Intervention zielte auf obengenannte Verhaltensweisen ab. In dieser Dissertation wird zunächst der Entwicklungsprozess der Intervention beschrieben (Kapitel 2) sowie die Dissemination der Förderungsmaßnahme über verschiedene Gesundheitsämter (Niederländisch: GGD; Kapitel 3). Darüber hinaus untersucht die Dissertation das Nutzerverhalten der Interventionsteilnehmer (z.B. Abbruchquote; Kapitel 4 und 5). In den letzten drei Kapiteln werden die Ergebnisse bezüglich der Effektivität (Kapitel 6 und 7) und Kosteneffektivität dargestellt (Kapitel 8).

Kapitel 1 gibt eine allgemeine Einleitung in das Thema dieser Dissertation. Dabei liegt der Schwerpunkt auf der Änderung von mehr als einem Gesundheitsverhalten (sogenannte multiple Verhaltensänderung) sowie der Maßschneiderung von Interventionsinhalten mittels computergestützten Expertensystemen (sogenanntes computer-tailoring). Des Weiteren wird der theoretische Rahmen der Studien - das I-Change Modell - vorgestellt. Daraus werden abschließend die Ziele und Forschungsfragen der Dissertation abgeleitet.

Kapitel 2 stellt das Studienprotokoll der Intervention vor, welches die einzelnen Entwicklungsschritte zur Fertigstellung der Intervention zur multiplen Verhaltensänderung, genannt meinGesundesVerhalten (Niederländisch: "mijnGezondGedrag"), dokumentiert. Ziel der web-basierten Lifestyle-Intervention war es, körperliche Aktivität und Obst- und Gemüsekonsum zu erhöhen, sowie den Alkoholkonsum zu reduzieren und mit dem Rauchen aufzuhören.

In Kapitel 3 wird die Disseminationsstrategie, die in der Studie benutzt wurde, beschrieben. Verschiedene niederländische Gesundheitsämter trugen mit der Verbreitung eines Gesundheitsfragebogens dazu bei, Teilnehmer für die Interventionsstudie zu gewinnen. Die Ergebnisse zeigen, dass diese Strategie eine brauchbare Methode war, um Erwachsene zu erreichen und die Intervention zu verbreiten. Ungefähr ein Drittel ( $\mathrm{c}$ 5.000) der Teilnehmer, die den OnlineGesundheitsfragebogen ausgefüllt haben, loggten sich ebenfalls in unser Programm ein. Andererseits brachen eine Vielzahl von Personen das Programm bereits während der Baseline-Erhebung ab und loggten sich eher selten zu einem späteren Zeitpunkt in das Programm ein. Dabei zeigte sich, dass Männer, ältere Teilnehmer, Teilnehmer mit einem höheren Bildungsstand und Teilnehmer mit einer gesünderen Lebensweise sowie einem niedrigeren Body Mass Index eher bereit waren, an der Intervention teilzunehmen. Das bedeutet, dass das Erreichen von Risikogruppen (zum Beispiel Personen, die wenig gebildet sind und/oder eine ungesunde Lebensweise haben) niedrig war.

In Kapitel 4 werden Ergebnisse einer Interventionsstudie vorgestellt, die das Nutzerverhalten der Teilnehmer (d.h. Beendigung/Vervollständigung der Intervention sowie späterer Drop-out) in Abhängigkeit von ihrer Interventionsgruppe untersucht. Teilnehmer nahmen entweder an einer simultanen oder einer sequentiellen Intervention teil. In beiden Interventionen waren die Abbruchraten während der Baselinemessung hoch. Während in der sequentiellen Gruppe 65,0\% der 
Teilnehmer die Intervention nicht komplett ausgefüllt hat, waren es in der simultanen Gruppe 77,1\%. In beiden Gruppen war das Geschlecht ein Prädiktor für ein frühzeitiges Abbrechen des Programms: Männer brachen das Programm eher ab als Frauen. Unter Teilnehmern, die sich nicht an zwei oder mehr Gesundheitsempfehlungen hielten, waren die Teilnehmer aus der simultanen Gruppe eher geneigt das Programm abzubrechen als Teilnehmer, die an der sequentiellen Intervention teilnahmen. Mögliche Gründe für die höheren Abbruchraten in der simultanen Gruppe könnten der erhöhte Zeitaufwand und Informationsüberfluss sein.

Kapitel 5 beinhaltet eine Studie, die einen weiteren Aspekt des Nutzerverhaltens untersucht. Dabei werden die individuellen Präferenzen für bestimmte Lebensweisen-Module innerhalb der multiplen Lifestyle-Intervention in den Schwerpunkt gerückt. In dieser Studie wurden nur Teilnehmer aus der sequentiellen Gruppe eingeschlossen. Während des ersten Besuchs (Baselinemessung) hatten Teilnehmer die Möglichkeit eine von fünf Modulen auszuwählen. Das Bewegungs-Modul war am beliebtesten, gefolgt von Rauch- und Obst-Modulen. Junge Erwachsene schienen das BewegungsModul und das Obst-Modul zu bevorzugen, wohingegen das Gemüse-Modul unter den älteren Erwachsenen beliebter war. Für das Alkohol-Modul und das Rauch-Modul wurde kein konsistentes Bild gefunden. Die Ergebnisse dieser Studie lassen vermuten, dass Bewegung als erste Verhaltensweise in eHealth-Interventionen genutzt werden kann, um auch andere Verhaltensweisen zu verändern (der sogenannte gateway effect).

In Kapitel 6 wird eine randomisierte kontrollierte Studie $(R C T ; n=448)$ vorgestellt, die die Effektivität einer web-basierten Alkoholintervention mit maßgeschneiderten Texten nach sechs Monaten testet. Diese Intervention zielte auf die Reduzierung von Alkoholkonsum bei Erwachsenen ab, die bereits bei der Baselinemessung einen erhöhten Alkoholkonsum aufwiesen. Zwei Tailoringsstrategien wurden miteinander verglichen: eine Abwechslung-Gruppe (das bedeutet, dass die Feedbacktexte in eine Reihe von Empfehlungen, die unterschiedliche Themen behandelten, aufgeteilt wurden, während der Teilnehmer das Programm ausfüllte) und eine summative Gruppe (das bedeutet, dass alle Empfehlungen auf einmal gezeigt wurden, nachdem der Teilnehmer alle Fragen innerhalb der Intervention beantwortet hatte). Bei der Analyse der Daten von Teilnehmern, die zu allen Messzeitpunkten vollständige Angaben hatten (sogenannte complete case-Analysen), zeigte sich, dass die Umsetzung von web-basierten, maßgeschneiderten Textelementen eine effektive Interventionsstrategie ist, um Alkoholkonsum bei Erwachsenen zu reduzieren. Dieser Effekt wurde wiederum nicht gefunden, wenn die fehlenden Werte anhand einer multiplen Imputationstechnik ersetzt wurden. Weiterhin zeigte sich, dass beide Tailoringsstrategien im Hinblick auf Alkoholreduzierung gleich effektiv waren. In Bezug auf die Bewertung des Programms zeigten sich zwischen den beiden Experimentalgruppen keine Unterschiede. Dennoch waren die Ausfallquoten in der Intervention, in der Fragen und Feedbacktexte abwechselnd gezeigt wurden, während der Nullmessung niedriger. Dies könnte dahingehend interpretiert werden, dass diese Intervention bevorzugt wurde.

Kapitel 7 beschreibt eine randomisierte kontrollierte Studie, die die Langzeiteffektivität der webbasierten Lifestyle-Intervention nach 12 und 24 Monaten bei Erwachsenen untersucht. Im Rahmen der Studie wurde ein Drei-Gruppen-Design umgesetzt: eine sequentielle Gruppe, eine simultane 
Gruppe und eine Kontrollgruppe. Die Ergebnisse zeigten, dass beide Tailoringsstrategien effektiv waren, einen gesunden Lebensstil zu fördern. Nach 12 Monaten zeigten sich signifikante Unterschiede in den Effekten der sequentiellen Intervention im Vergleich zur Kontrollgruppe, wobei sich die sequentielle Intervention als effektiver herausstellte $(E S=0,30)$. Nach 24 Monaten stellte sich die simultane Intervention als effektivste Interventionsgruppe heraus ( $E S=0,19$ ). Die vergleichbare Effektivität der beiden Interventionstypen legt nahe, dass Gesundheitsförderer beide Methoden benutzen können. Welche Methode besser ist, könnte vom Zielverhalten der Intervention abhängig sein.

In Kapitel 8 werden Ergebnisse der ökonomischen Evaluation der web-basierten maßgeschneiderten Lifestyle-Intervention beschrieben. Die Kosteneffektivität und die Nutzwertanalyse von beiden Tailoringsmethoden werden dargestellt. Die Ergebnisse zeigen, dass beide Programme kosteneffektiver waren als das Programm, an dem die Kontrollgruppe teilnahm. Teilnehmer der Kontrollgruppe erhielten eine kurze, maßgeschneiderte Übersicht über ihre Gesundheitsverhaltensweisen. Im Allgemeinen war die simultane Intervention am kosteneffektivsten, gefolgt durch die sequentielle Intervention. Bei inkrementellen Kosten von €4.594 pro Verbesserung der Lifestylescore (dadurch, dass sich der Teilnehmer an eine weitere Richtlinie hielt) war die sequentielle Gruppe wahrscheinlich am kosteneffektivsten; bei inkrementellen Kosten von €17.106 oder mehr war die simultane Version der web-basierten Intervention wahrscheinlich kosteneffektiver als die sequentielle Version. Mit Blick auf den Nutzwert bekam die Intervention, die die Kontrollgruppe erhalten hat, den Vorzug im Vergleich zu den beiden Lifestyle-Interventionen (sowohl sequentiell als auch simultan).

In Kapitel 9 werden die Ergebnisse der verschiedenen Studien dieser Dissertation (Kapitel 2 bis 8) diskutiert. Empfehlungen für Theorie, zukünftige Untersuchungen und die Praxis werden gegeben. Die Ergebnisse der Studien zeigen auf, dass es besonders wichtig ist, die Reichweite von web-basierten (multiplen) Lifestyle-Interventionen, die mehr als ein Verhalten ansprechen, weiter zu optimieren. Dabei sollte der Schwerpunkt sowohl darauf gelegt werden, das Interesse an solchen Interventionsmaßnahmen bei Erwachsenen zu wecken oder zu erhöhen als sie auch tatsächlich zu einer Teilnahme zu motivieren. Um dieses Ziel zu erreichen, sollten zunächst effektive Strategien zum ersten Gebrauch, zur Vollendung und zum wiederholten Gebrauch dieser Art Programme identifiziert werden. In multiplen Lifestyle-Interventionen könnte körperliche Aktivität als gateway behaviour dienen, um gesunde Lebensweisen via eHealth zu fördern. Zu guter Letzt waren beide web-basierten maßgeschneiderten Lifestyle-Interventionen effektiv und kosteneffektiv. Beide können deshalb zur Implementation benutzt werden.

Die Hauptschlussfolgerungen dieser Dissertation können folgendermaßen zusammengefasst werden: Die Interventionen haben positive Ergebnisse im Hinblick auf Reichweite, Bewertung, Effektivität und Kosteneffektivität gezeigt. Um mehr als ein Gesundheitsverhalten zu fördern, könnte eine Kombination der sequentiellen und der simultanen Tailoringsstrategie am besten geeignet sein. Andererseits leiden die Interventionen an hohem Drop-out, resultierend in einer hohen Anzahl fehlender Werte. Außerdem trat selektiver Ausfall auf. Aus diesen Gründen müssen die Ergebnisse mit 
Vorsicht interpretiert werden. Weitere Studien sind notwendig, um die niedrigen Teilnahme-Niveaus in web-basierten Interventionen zu verstehen und zu reduzieren. Außerdem sollte untersucht werden, welche statistischen Methoden, um mit fehlenden Werten umzugehen, am besten sind. 


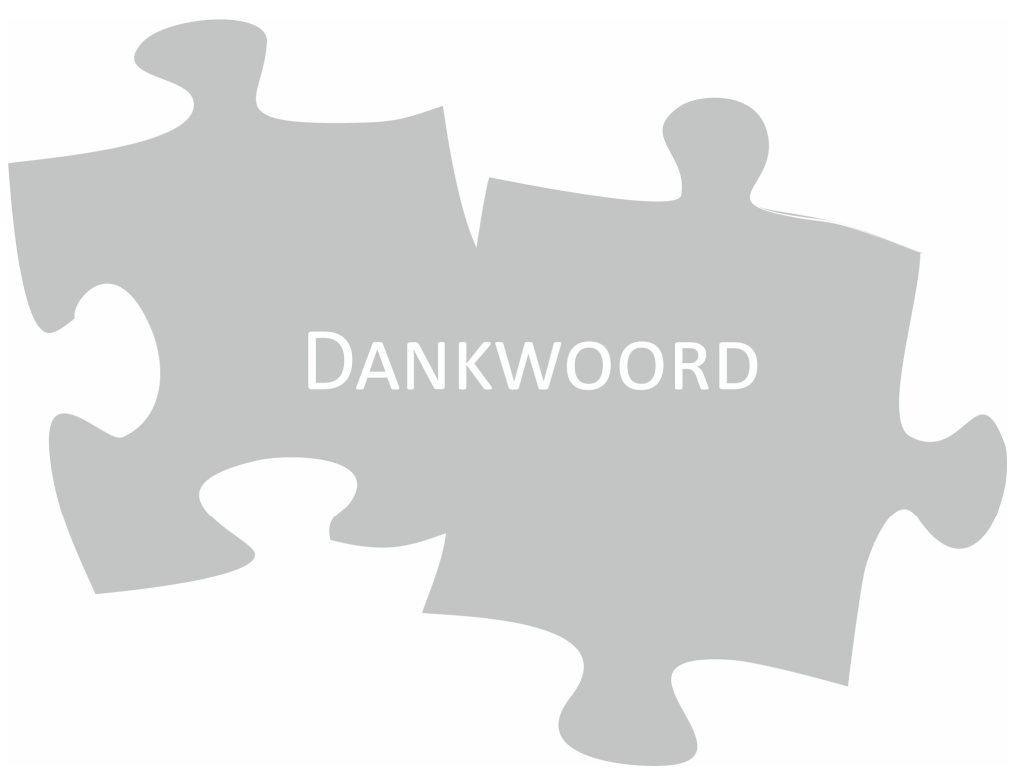



Waar de wetenschap altijd een puzzel met ontbrekende stukjes zal blijven, is dit proefschrift tot stand kunnen komen doordat hier wel alle puzzelstukjes op de juiste plaats vielen. Zonder de hulp van alle onderstaand genoemde personen en partijen, in het bijzonder mijn promotoren Hein en Stef, was dit mij niet gelukt. Eenieder heeft een bijdrage geleverd op zijn of haar eigen manier. Hartelijk bedankt!

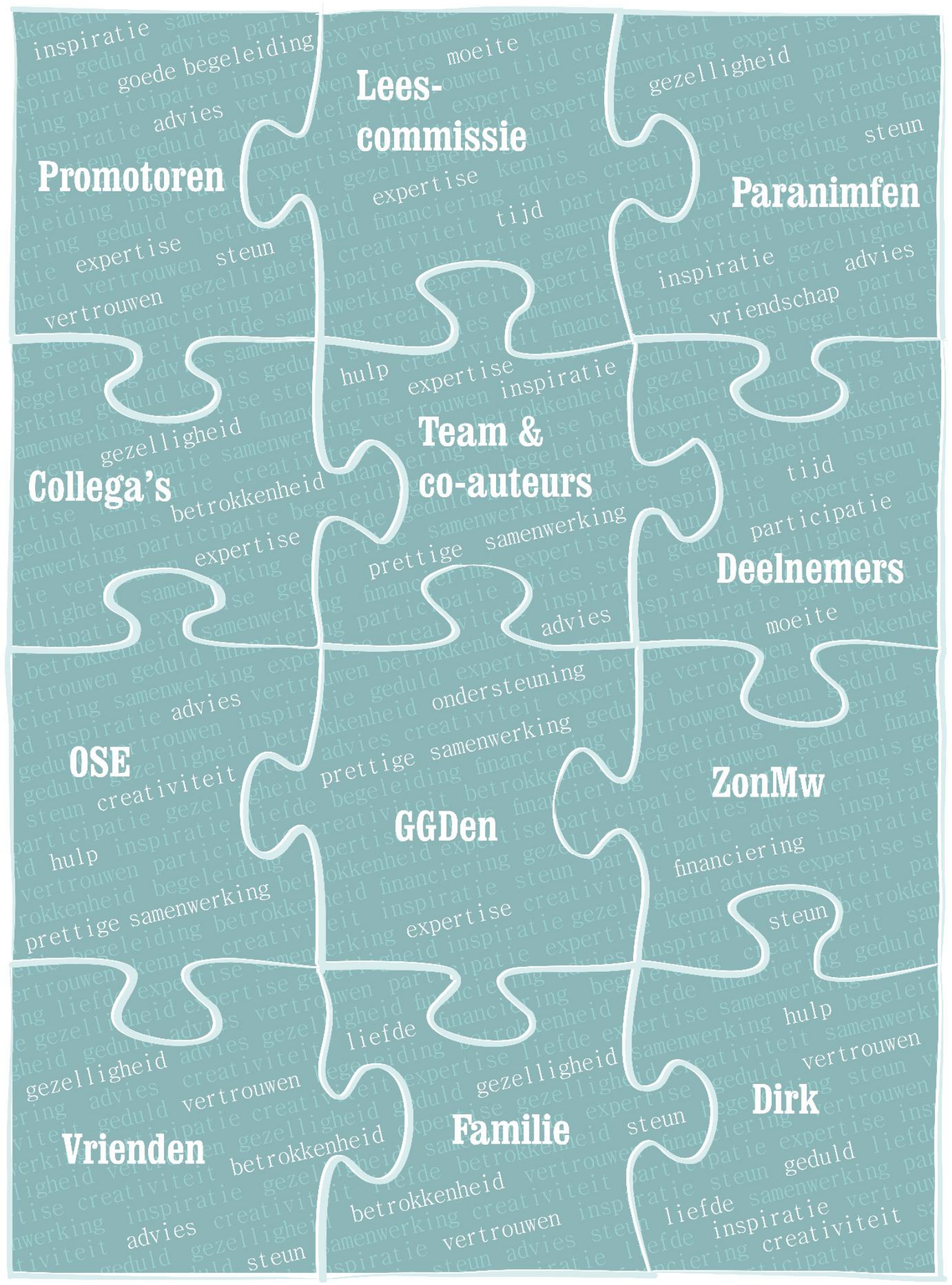





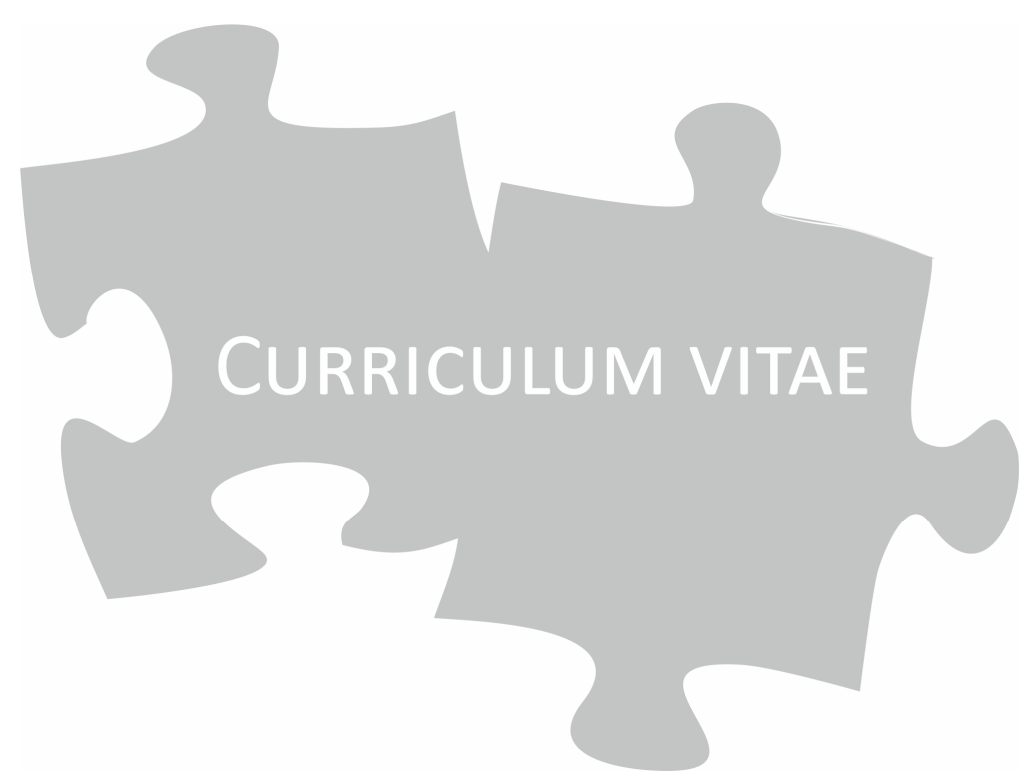



Daniela Schulz was born on 20 February 1984 in Duisburg (Germany). In 2004, she moved to Maastricht to study Health Sciences. During her studies, she went abroad to Gävle University (Sweden) to attend health psychology courses. She received her bachelor's degree in General Health Sciences in 2007. Afterwards, she moved to Berlin for a period of two months for an internship at Charité Berlin, Center of Human and Health Sciences, about paedophilia and child abuse. After that, she followed the Health Education and Promotion master at Maastricht University. She graduated in 2008 and received her master's degree in Public Health. In September 2008, she started working as a PhD candidate at the Department of Health Promotion of Maastricht University to conduct the research about computer-tailoring and lifestyle presented in this dissertation. During her PhD period, she obtained a grant from the European Health Psychology Society to work on an international project and visited the Freie Universität Berlin to develop a physical activity intervention delivered by tailored mobile phone short text messages. Moreover, Daniela developed a computer-tailored web-based intervention for problem drinkers in collaboration with the mental health care institution Mondriaan. As part of her job at Maastricht University, she developed a medication adherence programme for Vision2Health targeting patients with hepatitis C. Since the autumn of 2013, she has been working in the Catharina Hospital Eindhoven's department of quality and safety, as leader on a project to evaluate and improve the quality of care in the heart centre. Furthermore, Daniela works part-time as a researcher at the Department of Health Promotion of Maastricht University. 



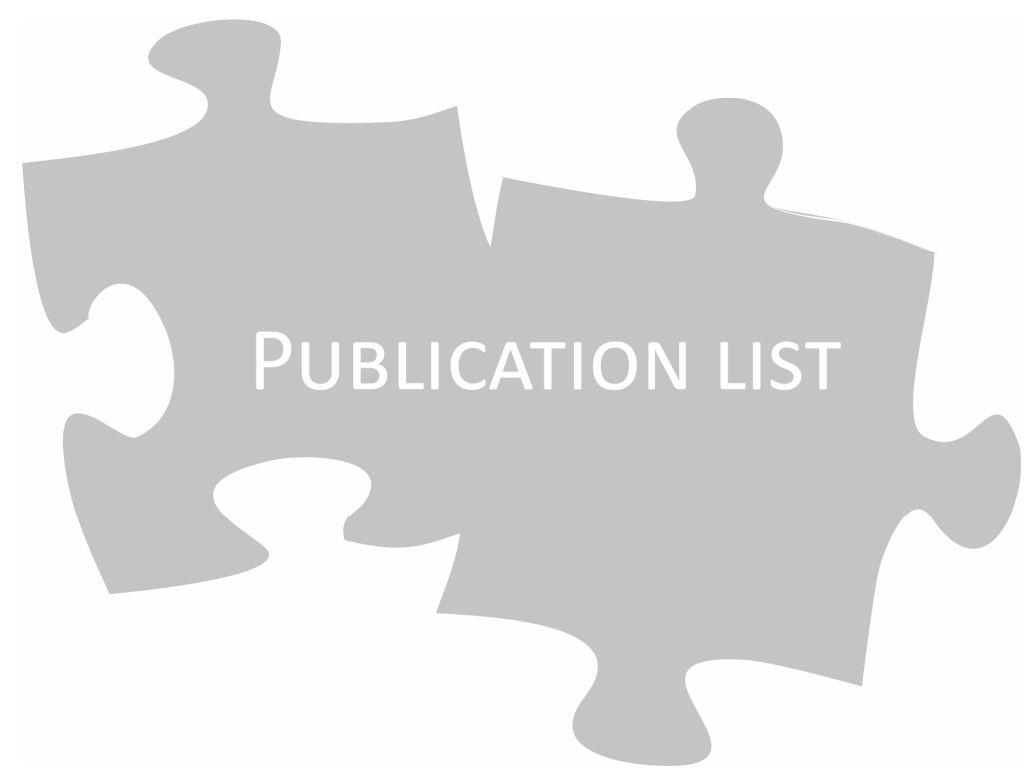





\section{D.D. $24^{\text {th }}$ March 2014}

\section{Published / accepted}

SCHULZ DN, KREMERS SP, DE VRIES H. Tailored eHealth lifestyle promotion: Which behavioural modules do users prefer? J Health Commun (in press).

SCHULZ DN, SMIT ES, STANCZYK NE, KREMERS SP, DE VRIES H, EVERS SMAA. Economic evaluation of a web-based tailored lifestyle intervention for adults: findings regarding cost-effectiveness and costutility from a randomized controlled trial. J Med Internet Res 2014; 16:e91.

SCHULZ DN, KREMERS SP, VANDELANOTTE C, VAN ADRICHEM MJG, SCHNEIDER F, CANDEL MJJM, DE VRIES H. Effects of a web-based tailored lifestyle intervention for adults: a two-year randomized controlled trial comparing sequential and simultaneous delivery modes. J Med Internet Res 2014; 16:e26.

SCHULZ DN, CANDEL MJJM, KREMERS SPJ, REINWAND DA, JANDER A, DE VRIES H. Effects of a webbased tailored intervention to reduce alcohol consumption in adults: randomized controlled trial. J Med Internet Res 2013; 15:e206.

SCHNEIDER F, SCHULZ DN, POUWELS LHL, DE VRIES H, VAN OSCH LA. The use of a proactive dissemination strategy to optimize reach of an Internet-delivered computer-tailored lifestyle intervention. BMC Public Health 2013; 13:721.

SCHULZ DN, KREMERS SP, DE VRIES H. Are the stages of change relevant for the development and implementation of a web-based tailored alcohol intervention? A cross-sectional study. BMC Public Health 2012; 12:360.

SCHNEIDER F, VAN OSCH LA, SCHULZ DN, KREMERS SP, DE VRIES H. The influence of user characteristics and a periodic email prompt on exposure to an internet-delivered computertailored lifestyle program. J Med Internet Res 2012; 14:e40.

SCHULZ DN, SCHNEIDER F, DE VRIES H, VAN OSCH LA, VAN NIEROP P, KREMERS SP. Program completion of a web-based tailored lifestyle intervention: differences between a sequential and a simultaneous approach. J Med Internet Res 2012: 14:e26.

SCHNEIDER F, VAN OSCH LA, KREMERS SP, SCHULZ DN, VAN ADRICHEM MJ, DE VRIES H. Optimizing diffusion of an online computer tailored lifestyle program: a study protocol. BMC Public Health 2011; 11:480.

SCHULZ DN, KREMERS SP, VAN OSCH LA, SCHNEIDER F, VAN ADRICHEM MJ, DE VRIES H. Testing a Dutch web-based tailored lifestyle programme among adults: a study protocol. BMC Public Health 2011; 11:108.

\section{Submitted / under review}

STANCZYK NE, SMIT ES, SCHULZ DN, BOLMAN C, MURIS J, DE VRIES H, EVERS SMAA. Examining the cost-effectiveness and cost-utility of a video-based and text-based computer-tailored lifestyle intervention for smoking cessation. 



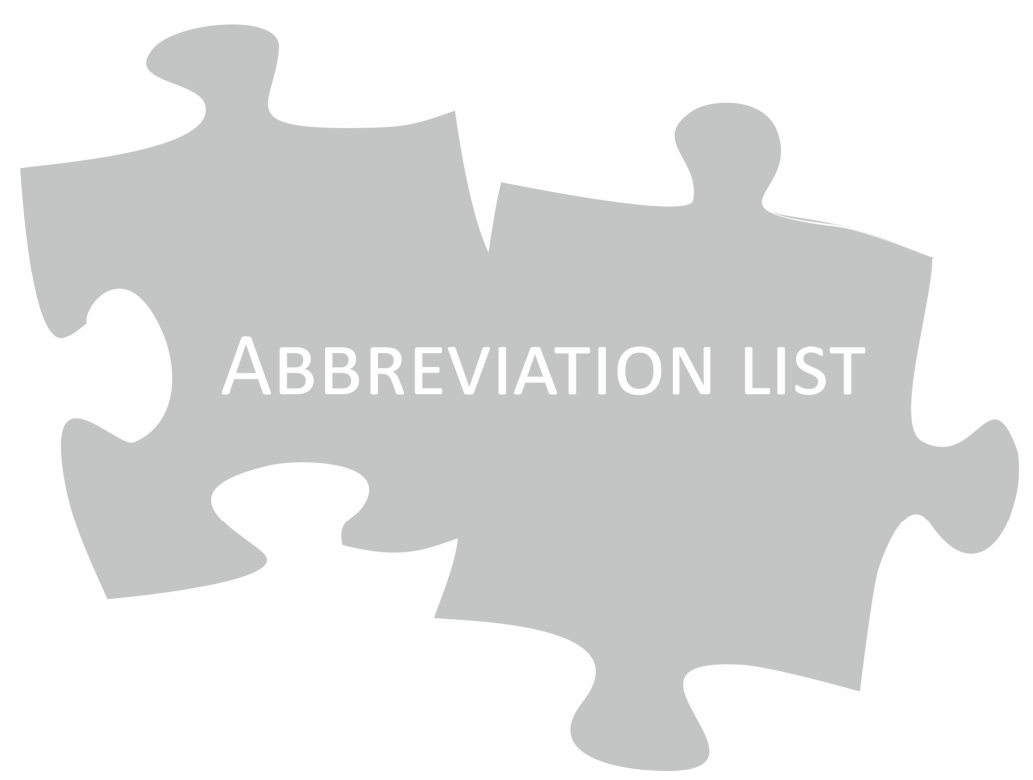





\begin{tabular}{|c|c|}
\hline ANOVA & ANalyses Of VAriance \\
\hline AUDIT & Alcohol Use Disorder Identification Test \\
\hline $\mathrm{BMI}$ & Body Mass Index \\
\hline $\mathrm{CC}$ & Control Condition \\
\hline CES-D10 & 10-item Center for Epidemiologic Studies Depression scale \\
\hline CONSORT & CONsolidated Standards Of Reporting Trials \\
\hline COPD & Chronic Obstructive Pulmonary Disease \\
\hline CVD & CardioVascular Diseases \\
\hline DF & Degrees of Freedom \\
\hline ES & Effect Size \\
\hline FCC & Fully Complete Cases \\
\hline $\mathrm{FFQ}$ & Food-Frequency Questionnaire \\
\hline GP & General Practitioner \\
\hline HRA & Health Risk Appraisal \\
\hline ITT & Intention-To-Treat \\
\hline $\mathrm{K} 10$ & Kessler Psychological Distress Scale (10 items) \\
\hline $\mathrm{LFCl}$ & Lifestyle Factor Change Index \\
\hline LFS & Lifestyle Factor Score \\
\hline LOCF & Last Observation Carried Forward \\
\hline $\mathrm{Ml}$ & Multiple Imputations \\
\hline OR & Odds Ratio \\
\hline QALYS & Quality Adjusted Life Years \\
\hline QFV & Quantity-Frequency-Variability \\
\hline $\mathrm{RCT}$ & Randomized Controlled Trial \\
\hline RE-AIM & Reach Effectiveness Adoption Implementation Maintenance \\
\hline $\mathrm{RHA}$ & Regional Health Authorities \\
\hline SD & Standard Deviation \\
\hline
\end{tabular}


SeqC

SES

$\operatorname{Sim} C$

SMS

SQUASH

$\mathrm{SRHI}$

TIA

TPB

WTP
Sequential Condition

SocioEconomic Status

Simultaneous Condition

Short Message Service

Short QUestionnaire to ASsess Health-enhancing physical activity

Self-Report Habit Index

Transient Ischemic Attack

Theory of Planned Behaviour

Willingness To Pay 

